\title{
Truck Technology Efficiency Assessment (TTEA) Project Final Report
}

\section{November 2012}

\section{Prepared by}

Tim LaClair, Zhiming Gao, Adam Siekmann (ORNL)

Joshua Fu, Jimmy Calcagno, Jeongran Yun (University of Tennessee, Knoxville)

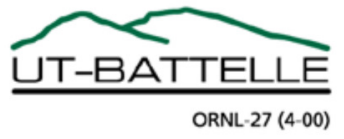



ORNL/TM-2012/586

Energy and Transportation Science Division

\title{
TRUCK TECHNOLOGY EFFICIENCY ASSESSMENT (TTEA) PROJECT FINAL REPORT
}

\author{
Tim LaClair \\ Zhiming Gao \\ Adam Siekmann
}

Date Published: November 6, 2012

\author{
Prepared by \\ OAK RIDGE NATIONAL LABORATORY \\ Oak Ridge, Tennessee 37831-6283 \\ managed by \\ UT-BATTELLE, LLC \\ for the \\ U.S. DEPARTMENT OF ENERGY \\ under contract DE-AC05-00OR22725
}




\section{Table of Contents}

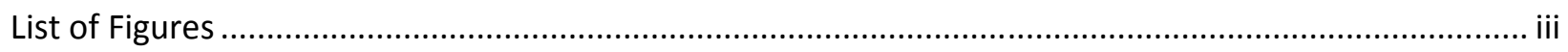

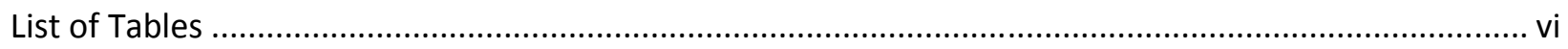

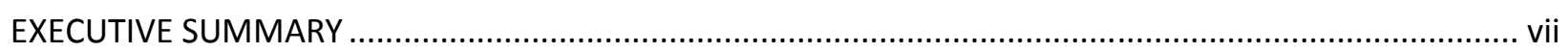

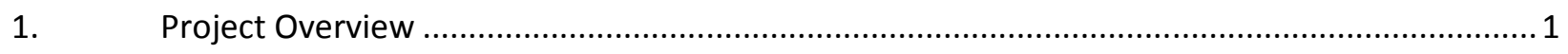

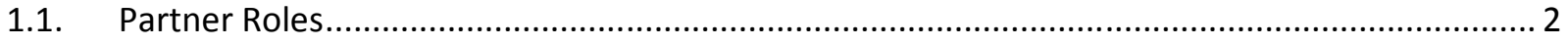

2. Vehicle Efficiency Modeling Methodology and the Tractive Energy Model ............................ 3

2.1. Calculation of Energy Savings Due to Vehicle Efficiency Technologies ................................... 10

2.2. Validation of the Tractive Energy Method and Concluding Remarks Concerning its

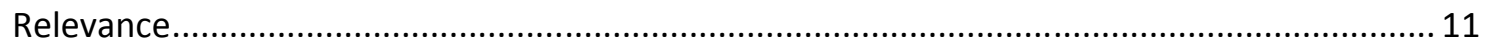

3. Preparation of Data Files from the Heavy Truck Duty Cycle (HTDC) Project........................... 16

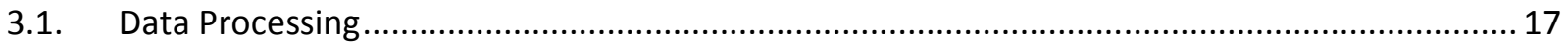

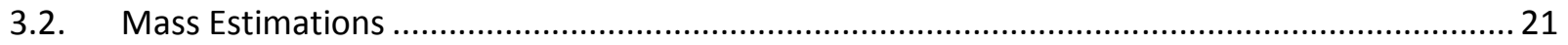

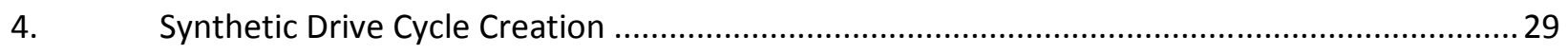

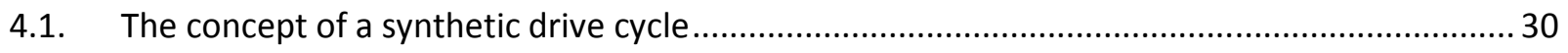

4.2. The Drive Cycle Generation (DCGen) Tool for developing a synthetic drive cycle .................... 31

4.3. Validating the Use of a Synthetic Drive Cycle and the Tractive Energy Model for Predicting the Fuel Efficiency Benefits from Advanced Vehicle Technologies .......................................... 37

5. Tractive Energy Analysis Results Based on the Synthetic Drive Cycle ....................................43

5.1. The synthetic drive cycle corresponding to the overall fleet usage ...................................... 43

5.2. Tractive energy reductions and fuel savings associated with vehicle efficiency technologies .. 48

5.3. Average fuel savings for the overall fleet usage ................................................................... 51

5.4. Summary of the tractive energy model results for the class 8 tractor-trailer application

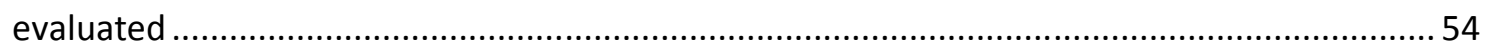

5.5. Consideration of the accuracy of using a substitute drive cycle as opposed to the synthetic

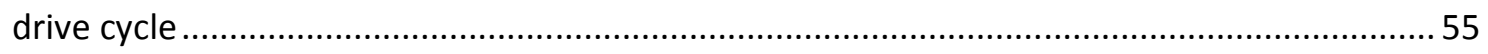

6. $\quad$ EPA MOVES (Motor Vehicle Emission Simulator) Model Analysis ..........................................57

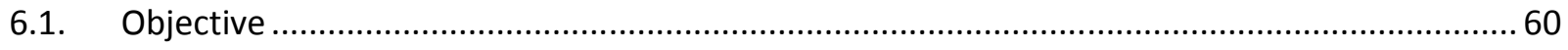

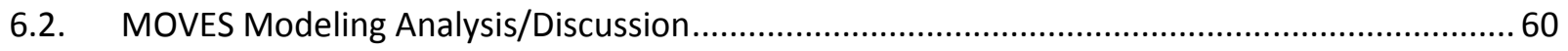

6.2.1. MOVES mean base rates for running exhaust emissions ............................................... 60

6.2.2. Emissions calculation procedure using the MOVES method .........................................61 
6.2.3. Comparison of MOVES predictions with measured emissions data from one of the HTDC test trucks.

6.2.4. Validation of the synthetic cycle methodology for MOVES simulations: comparison of results using a synthetic drive cycle that represents a full day of measured drive cycle data.

6.2.5. Evaluation of the emissions reductions from advanced efficiency technologies for the overall HTDC fleet usage using MOVES

6.2.6. Comparison of results using an alternative drive cycle with those from the synthetic cycle.....

6.2.7. Comparison of emissions estimates for synthetic and real drive cycles versus the default MOVES drive cycles.....

6.3. Summary and Conclusions of the MOVES analysis

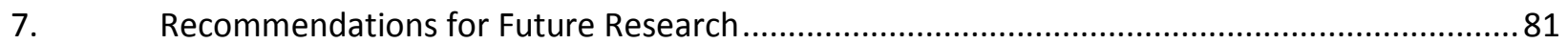

APPENDIX - Table of the synthetic drive cycle representing the full usage of HTDC drive data 


\section{LIST OF FIGURES}

Figure 1 Drive cycle example to indicate the periods of tractive energy savings (in black) when aerodynamic drag is reduced for a conventional vehicle.

Figure 2 The aerodynamic drag power during periods of positive tractive power for the drive cycle shown in Figure 1. The integral of this power represents the aerodynamic drag contribution to the total driving tractive power.

Figure 3 Periods of active braking during the drive cycle.

Figure 4 The braking power, which represents the maximum recoverable power with regenerative braking.

Figure 5 Comparison between the driving tractive power calculated using the tractive energy method, the engine power estimated from the tractive power, and the measured engine power during a measured drive cycle.

Figure 6 Comparison of the calculated tractive energy and the cumulative fuel consumption (measured) during periods of positive tractive power during a measured drive cycle. . 13

Figure 7 Cross-plot of the cumulative fuel consumption and the driving tractive energy results.

Figure 8 Comparison of the engine power requirement predicted with the tractive energy model and Autonomie for the first portion of the drive cycle.

Figure 9 Comparison of fuel consumption predicted by the tractive energy model and by Autonomie.

Figure 10 Comparison of the measured elevation and USGS elevation data for the same route (based on a lookup using the measured position).....

Figure 11 Comparison of the grade determined from raw elevation data and after filtering, for two time segments.

Figure 12 Plot of the tractive energy curves, based on measured engine data and determined from the drive cycle data, as used in the mass estimation. This sample case shows a mass change occurring at about 8 hours that was not properly identified by the automated calculation.

Figure 13 Plot of the tractive energy curves after the corrections were completed in the mass calculation.

Figure 14 Plot showing the measured weight data during a day with two load changes. .............26

Figure 15 Distribution of masses for all segments of travel in the HTDC project. ........................ 27

Figure 16 Adjusted mass distribution, based on a $12.65 \%$ increase compared to the original

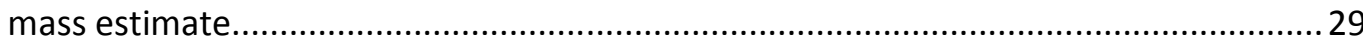

Figure 17 Bivariate histogram of the velocity and acceleration for a day of driving.................... 32

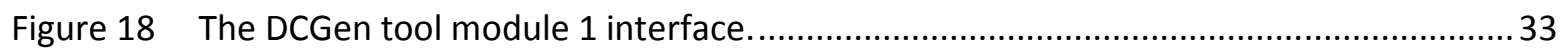

Figure 19 Difference and segment histograms during the creation of a synthetic drive cycle. ..... 34

Figure 20 Plot of a single drive segment in DCGen tool program module two, showing the

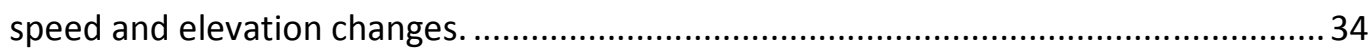

Figure 21 DCGen tool, second module segment search interface............................................. 34 
Figure 22 Manually-generated DCGenT synthetic plot........................................................... 35

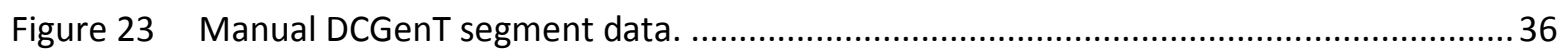

Figure 24 Manual DCGenT synthetic histogram...................................................................... 36

Figure 25 Driving data used to develop the validation synthetic drive cycle. ................................37

Figure 26 Synthetic drive cycle developed from the driving data presented in Fig. 23.................38

Figure 27 Bivariate histogram of the original driving data and of the synthetic drive cycle

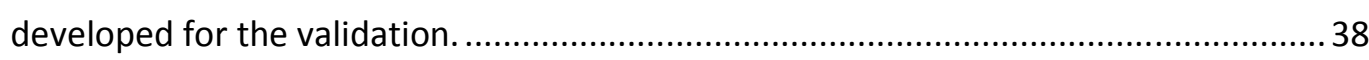

Figure 28 Original and synthetic histograms shown with the scale modified to highlight the low speed operating conditions.

Figure 29 Relative Fuel Savings estimates based on the tractive energy model for (a) the original drive cycle and (b) the synthetic drive cycle. The variations evaluated were for a mass reduction of $2000 \mathrm{~kg}$, a reduction in $C_{R R}$ by 0.0015 and a $10 \%$ reduction in $C_{d}$. The efficiency of the regenerative braking system is assumed to be $80 \%$.

Figure 30 Comparison of the speed vs. acceleration distributions for the low, medium and high mass operating conditions.

Figure 31 The main synthetic drive cycle developed for the TTEA project, representing the overall usage of the HTDC fleet.

Figure 32 Comparison of the distribution of the synthetic drive cycle and the original histogram containing all of the data from the medium mass operation.

Figure 33 Modified synthetic drive cycle, with the proper ratio of idle time for the overall average. The cycle duration is 3448 seconds.

Figure 34 Fuel savings estimate for combinations of advanced efficiency technologies for the low mass case.

Figure 35 Fuel savings estimate for combinations of advanced efficiency technologies for the medium mass case.

Figure 36 Fuel savings estimate for combinations of advanced efficiency technologies for the high mass case.

Figure 37 Fuel savings estimate for combinations of advanced efficiency technologies for the combined usage in the HTDC fleet.

Figure 38 Comparison of the histogram for substitute measured drive cycle to that of the overall usage for the HTDC fleet.

Figure 39 Fuel savings estimate using the substitute measured drive cycle. .................................57

Figure 40 Measured dynamometer hub speed data from the WVU dynamometer testing with the ORNL4LS drive cycle.

Figure 41 Nitrogen oxide emissions measured during the dynamometer test and the mean base rates predicted by MOVES using speed data from the ECU.

Figure 42 Carbon monoxide emissions measured during the dynamometer test and the mean base rates predicted by MOVES using speed data from the ECU. .

Figure 43 Gaseous hydrocarbon emissions measured during the dynamometer test and the mean base rates predicted by MOVES using speed data from the ECU.

Figure 44 Energy consumption measured during the dynamometer test and the mean base rates predicted by MOVES using speed data from the ECU. 
Figure 45 MOVES default cycles for average speeds $87.2 \mathrm{~km} / \mathrm{h}(54.2 \mathrm{mph})$ and $95.6 \mathrm{~km} / \mathrm{h}(59.4$ $\mathrm{mph}$ )......

Figure 46 Operating mode bin distribution for the original, synthetic, and MOVES average speed cycles..... 


\section{LIST OF TABLES}

Table 1: $\quad$ Comparison of results predicted with Autonomie to those from the tractive energy model.

Table 2: Data contained in the Excel spreadsheet during the calculation of the mass for the case shown in Fig. 10.

Table 3: Data used for the mass adjustment.

Table 4: Comparison of results for the energy loss factors in the tractive energy model, for the original and synthetic drive cycles from the validation case.

Table 5: Default values for parameters used in the tractive energy analysis...................................... 42

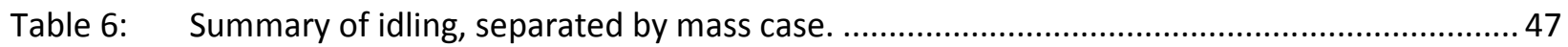

Table 7: Intermediate results of the tractive energy analysis for the low mass case, using the synthetic drive cycle. The results are based on the default model parameters shown in Table 4.

Table 8: $\quad$ Predicted driving tractive energy and contributions from energy loss factors for the medium mass case, using the synthetic drive cycle.

Table 9: Driving tractive energy and contributions from the different energy loss factors for the high mass case, based on the synthetic drive cycle.

Table 10: Distance-normalized tractive energy factors from the tractive energy analysis for each mass case, and the combined result for the full fleet.

Table 11: Distance traveled in each mass case.

Table 12: The contributions to the driving tractive energy from each energy loss factor for the overall, combined fleet usage, based on the synthetic drive cycle.

Table 13: The contributions to the driving tractive energy from each energy loss factor for the overall, combined fleet usage, based on the synthetic drive cycle. 56

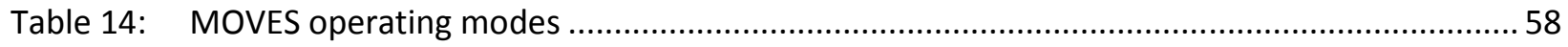

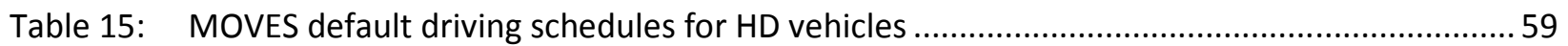

Table 16: Comparison of total emissions and energy consumption results from dynamometer measurements and MOVES predictions.

Table 17: Summary of MOVES predicted emissions for the validation synthetic cycle case, with a comparison of the original and synthetic drive cycle results.

Table 18: Emissions reductions associated with different advanced efficiency technologies as predicted by MOVES using the synthetic drive cycle representing the HTDC driving data.... 73

Table 19: Comparison of MOVES-predicted emissions using the synthetic drive cycle with those calculated using a substitute measured drive cycle.

Table 20: Comparison of the overall usage synthetic drive cycle and the MOVES default drive cycle based on the average speed of the synthetic cycle. 


\section{EXECUTIVE SUMMARY}

Medium- and heavy-duty trucks and buses are responsible for over $28 \%$ of the energy used and emissions generated in highway transportation, and class 8 tractor-trailers operating in long-haul and regional cargo transport are responsible for about $75 \%$ of all fuel consumed by commercial trucks. The vehicle miles traveled (VMT) for trucks is expected to increase at a rate significantly outpacing passenger VMT growth, which will result in a steady rise in the percentage of energy consumption (and emissions) attributable to trucks over the coming decades. These facts have sparked significant recent interest in truck fuel efficiency in the transportation community.

Although fuel economy regulations in the United States have historically focused on passenger cars, recent legislation requiring new standards for fuel economy in medium- and heavy-duty trucks aims to increase the efficiency of trucks as well. The development of regulations for truck fuel efficiency is quite challenging, however, since vehicle usage and configurations vary substantially among the very diverse set of trucking applications. Fuel economy is very strongly linked to the particular drive cycles followed by a given truck, as are the gains in efficiency that can realized by implementing new technologies. As demonstration of this fact, a technology that provides significant fuel efficiency gains for one trucking application may yield little improvement or could even be detrimental to fuel economy in a different trucking application. It is therefore critical that the usage of each application be well understood and carefully evaluated to select the set of technologies that can provide the greatest benefits for each application.

Although it is well known that drive cycle data representative of a vehicle or fleet's usage is crucial for an accurate evaluation of fuel economy benefits or to identify an optimum set of technologies to reduce fuel consumption, detailed drive cycle data of trucks are not readily available for many applications. The Oak Ridge National Laboratory (ORNL) has collected a rather extensive set of truck duty cycle data in an effort to characterize the usage of several trucking applications. During the first phase of ORNL's duty cycle data collection activities, drive cycle measurements were made from six tractor-trailers during normal operations in a regional commercial shipping fleet, operating primarily in the southeastern U.S., for a period of a full year. This data, contained in the Heavy Truck Duty Cycle (HTDC) project database, was analyzed in detail for the current research effort in the Truck Technology Efficiency Assessment project. Based on the high fidelity HTDC duty cycle data, the results of this study are therefore highly representative of tractor-trailer operations for this type of regional, freeway-dominant trucking application.

The TTEA project used data collected in the prior study to develop a drive cycle with the same statistical characteristics for the accelerations, speeds and associated power from the engine as those for the complete set of data from the HTDC database. This drive cycle, which is highly representative of the complete usage of these vehicles, is a synthesis of all of the information contained in the original data set, but the resulting synthetic drive cycle is less than one hour in length and is thus appropriate for use in vehicle performance models or in fuel economy or emissions testing. A rigorous approach and 
software tools were developed for generating the synthetic drive cycle, and the methodology was validated against measured fuel consumption data as part of this project.

The mass of the vehicles during each segment of travel was also extracted from the data to determine the load distribution as a function of distance traveled. The drive cycle and load results were then used to quantify the energy use attributed to the multiple energy loss factors associated with heavy duty truck operation and to assess the fuel savings potential of implementing various advanced efficiency technologies. This research employed a vehicle tractive energy analysis to explore measured, real-world drive cycle data in order to quantify the effectiveness of technologies that impact the fuel consumption of heavy duty trucks. A detailed analysis of the complete set of measured drive cycle data was performed to develop a better understanding of how advanced efficiency technologies impact the fuel consumption and emissions of class 8 tractor-trailers in regional truckload operations.

Although the class 8 tractor-trailer application considered for this study is the most well understood of all trucking applications, results from this study still provided a few surprises and interesting results:

1. First, the duration of engine idle operation was $49.9 \%$ of the total engine run time for the fleet studied. This idling was responsible for the consumption of approximately 22,000 liters (5830 gallons) of diesel fuel from the six trucks during a year of data collection, which increased the average fuel consumption rate by approximately $1.9 \mathrm{~L}$ for each $100 \mathrm{~km}$ traveled and corresponds to about $5 \%$ of the total fuel consumption for this trucking fleet. The vast majority of the idling (47.2\%) occurred during periods exceeding 5 minutes, indicating that stops in traffic were $2.7 \%$ of the idling, and the majority of the long term idling took place over several hours at a time. It is estimated that the use of an auxiliary power unit (APU) on each truck could have saved approximately 15,800 L (4150 gallons) of diesel during the year for the six trucks. Engine startstop technology to reduce idling in congestion and at stop lights, however, would yield relatively small benefits for this trucking application.

2. The tractive energy analysis indicated that about $10 \%$ of the tractive energy losses are due to braking or engine braking, which represents a significant quantity of energy available for recovery using a regenerative braking system. Although only some fraction of this energy could be recovered with a hybrid powertrain (so the fuel savings benefit with a hybrid system would be less than the $10 \%$ ), this magnitude of energy availability is rather surprising for a fleet that operates mostly on freeways with an average driving speed of $95 \mathrm{~km} / \mathrm{hr}$ ( $59 \mathrm{mph}$ ). It is considered likely that many regional tractor-trailer fleets that drive more regularly in offfreeway operations could likely exceed a break-even point for the benefits of a hybrid system incorporating regenerative braking.

3. It was rather unexpected that the statistical distribution of accelerations and speeds changed very little with changes to the vehicle load. In developing the synthetic drive cycle from the complete set of measured driving data, several load categories were evaluated. The acceleration-speed distributions for low load (<20,000 kg [44,000 pounds]), medium load $(10,000$ to $28,180 \mathrm{~kg}[62,000$ pounds]) and high loads ( $>28,180 \mathrm{~kg})$ were all very similar, even though a reduced load enables the trucks to accelerate more quickly due to an effectively higher 
power-to-weight ratio. This similarity in drive cycle for all load ranges simplifies the analysis since it is not necessary to have separate drive cycles for different loads.

4. Concerning the distribution of loads carried, it was found that the average mass of the loaded vehicles from this study was only about $24,750 \mathrm{~kg}$ (54,500 pounds). It is frequently assumed that tractor-trailer freight operations consist of fully loaded tractor-trailers operating at close to maximum load, but the distribution of loads carried by the study fleet covered the full range from empty to full. This is important not only in quantifying the benefits of efficiency technologies, but also in considering potential policy actions that might be pursued to increase the operational efficiency of our nation's freight delivery.

The tractive energy analysis estimated approximately $6.6 \%$ in fuel savings possible, on average, from a $1.5 \mathrm{~kg} /$ ton reduction in the coefficient of rolling resistance, a level that is typically achieved when replacing conventional dual tires with new generation wide-base single (NGWBS) tires. For a $10 \%$ reduction in aerodynamic drag coefficient, which can be achieved when implementing many of the aerodynamic reduction devices currently available, the fuel savings is estimated to be $3.9 \%$. A mass reduction of $2000 \mathrm{~kg}$, through the use of lightweight materials in the truck construction, is estimated to provide fuel savings of approximately $4.1 \%$ assuming that the same loads would be carried. These savings are substantial and can provide not only reductions in trucking operational costs, but can help the nation to reduce its reliance on foreign oil, thereby improving our energy security.

The MOVES analysis conducted in this project confirmed that the synthetic drive cycle methodology provides consistent results to those that would be predicted when using the original driving data. MOVES predictions of the emissions reductions possible with the implementation of various combinations of advanced efficiency technologies showed that sizable emissions reductions are achievable for this vehicle application, particularly for $\mathrm{NO}_{x}, \mathrm{PM}$ and $\mathrm{CO}_{2}$ emissions. Predicted reductions of $\mathrm{HC}$ and $\mathrm{CO}$ emissions were very limited based on the MOVES results, but it was found that these emissions did not match measured emission levels very well, so these predictions are somewhat questionable.

Although there are certainly differences among class 8 tractor-trailer fleets, this study provides valuable insight into the energy and emissions reduction potential that various technologies can bring in this important application. The synthetic drive cycle developed from a full year of measured data on six trucks from the fleet studied is believed to be quite typical of class 8 tractor-trailer operations.

This study supports the U.S. Department of Transportation (DOT) in its mission to address energy efficiency and conservation, energy security, global climate change in transportation, and related environmental impacts. For the development of truck fuel efficiency standards, in particular, the authors of this report believe that the results of this research will be particularly useful in evaluating the fuel economy and emissions reductions benefits that can be expected in this important class of vehicles. The same analysis approach can also provide further insight in other vehicle applications, and it is hoped that the approach developed in this study will be useful in characterizing vehicle usage and energy savings potential in other trucking applications. 


\section{Project Overview}

This project consists primarily of a detailed analysis of a large data set of duty cycle measurements collected from a class 8 trucking fleet operating in the southeastern United States. The primary objective of the TTEA project was to quantify the potential fuel consumption and emissions reductions that can be achieved using advanced energy efficiency technologies for a regional class 8 trucking operation, including combinations of these technologies. To evaluate the potential fuel savings and emissions reductions that these technologies can bring, a unique analysis approach was followed. First, a synthetic drive cycle that is highly representative of the complete operation of the fleet was developed, based on a statistical evaluation and analysis of the entire set of drive cycle measurements collected during a previous Oak Ridge National Laboratory (ORNL) study. The synthetic drive cycle is appropriate to use both for modeling and testing purposes, and the results when using this cycle should very closely match the performance for the overall trucking fleet since the drive cycle measurements were collected from six trucks during a full year of normal operations in the fleet and are therefore very representative of the fleet's overall operations. To evaluate the energy savings that can be achieved with various energy efficiency technologies, a tractive energy analysis was employed using the synthetic drive cycles developed. An additional result of the analysis of the drive cycle data included a determination of the mass distribution of the vehicles during the fleet's operations, and this distribution was also incorporated in the analysis for assessing the fuel and emissions savings potential for the fleet. The final result of this study is an assessment of the overall potential for fuel savings and emissions reduction that can be expected if any combination of the technologies considered were to be implemented in the fleet. This data and the analysis approach employed can assist the DOT in quantifying the improvements in fuel economy that can be realized in the U.S. trucking fleet through the implementation of specific technology options. Furthermore, this assessment enables a detailed evaluation of the costs associated with implementing the technology needed to achieve specific fuel efficiency targets for class 8 tractor-trailers.

For the emissions evaluations, the U.S. Environmental Protection Agency's (EPA's) MOVES (Motor Vehicle Emission Simulator) model was used to characterize the emissions of the trucks. Parameters that are relevant to the vehicles tested were used in the analysis, and the MOVES model was run using both the default drive cycles in MOVES for class 8 tractor-trailers and the synthetic drive cycle developed in this project, which represents the detailed usage of the fleet. The MOVES model results were also compared directly to emissions measurements that were conducted as part of ORNL's data collection activities in the previous research effort. In this way, the relevance of the MOVES model was evaluated. It is therefore expected that these results can be used by the EPA in making improvements to the MOVES software.

The development of a synthetic drive cycle from extensive drive cycle measurements is a new approach for generating a drive cycle that is highly representative of the overall operations. Due to the manner in which the synthetic drive cycle is generated, the results from an analysis or test when using it will be 
extremely similar to those that would be achieved if the complete data set were analyzed or tested. Therefore, when a large data set representative of the overall fleet operations is used as the basis for developing the synthetic drive cycle, the cycle can be used to estimate, with high accuracy, the fuel savings benefits that would be attainable in the fleet if particular technologies are deployed. The accuracy of using a synthetic drive cycle as a substitute for a broader data set that the synthetic drive cycle represents was demonstrated in this project through a validation study that examined a full day's drive cycle. The predicted energy savings based on the 30-minute synthetic drive cycle matched the results from the full cycle to within $1 \%$ for each of the evaluations considered. The synthetic drive cycle approach can be used at any scale to develop characteristic drive cycles for any fleet of similar vehicles. The tools developed for this project can be of great benefit to policy makers in evaluating the benefits of advanced vehicle technologies at the national level and for different trucking applications, although specific drive cycle data needs to be available to characterize each application.

The advanced efficiency technologies evaluated for this project include regenerative braking (hybrid systems), aerodynamic drag reduction devices, low rolling resistance tires, vehicle mass reduction, and idle reduction technologies. The method can also be used to quantify and compare fuel savings due to improvements in engine thermal efficiency and reductions in accessory power loads. To evaluate the fuel saving potential of these technologies, the study analyzed existing data that was collected from six tractor-trailers during a period of over one year as part of a DOE-sponsored activity at ORNL (the Heavy Truck Duty Cycle, or HTDC, project) [1]. Inherent in these data are the impact of driver behavior, congestion, and other real-world issues associated with heavy-duty truck operations. Accurate duty cycle data-speed, elevation and load histories as a function of time-are critical to determining fuel economy and emissions, and the fuel savings potential of advanced efficiency technologies also depends very strongly on how the vehicle is driven. Therefore, it is essential that relevant, real-world duty cycle data be used to perform any evaluations regarding fuel savings potential. ORNL's HTDC database is believed to contain the most comprehensive measurement of real-world duty cycle information available and serves as a high-fidelity representative set of duty cycle information for the class 8 regional tractor-trailer application evaluated in this study.

\subsection{Partner Roles}

The TTEA project was completed as a joint research effort between ORNL and the University of Tennessee, Knoxville (UTK). ORNL, which is the largest Department of Energy (DOE) science laboratory, was the primary technical lead and project manager for the TTEA project, while UTK provided technical and analysis support for the MOVES modeling activities.

ORNL, through its Center for Transportation Analysis (CTA), has extensive background in the collection and analysis of truck drive cycle data and this work extends the analysis of data contained in the HTDC database. For the project, ORNL was responsible for the processing of all drive cycle data files, software development for creation of the synthetic drive cycles, all drive cycle development, and fuel economy modeling using the tractive energy model and Autonomie software. ORNL also conducted additional emissions analysis using measured emissions data and an empirical modeling approach. 
The UTK Department of Civil and Environmental Engineering has specific expertise and conducts research in climatic change, environmental impact assessments, transportation and air quality, and vehicle emissions. UTK's primary responsibility in the project was to perform all of the modeling and analysis using the EPA MOVES model, including the comparison of results with measured emissions data.

\section{Vehicle Efficiency Modeling Methodology and the Tractive Energy Model}

Before moving into the details of the data processing and synthetic drive cycle creation performed for the project, it is instructive to first provide a summary of the tractive energy analysis approach used to evaluate the fuel saving potential of the advanced efficiency technologies of the class 8 tractor-trailers. The full analysis methodology is described in detail in [2], but a general discussion of the method is given below and the fundamental equations used are presented here.

An accurate prediction of the fuel savings achievable in a fleet or for a particular trucking application when various fuel efficiency technologies are implemented can be very challenging, particularly when considering combinations of technologies. Differences in highway vehicle usage can strongly influence the benefits realized with any technology, which makes generalizations about fuel savings inappropriate for different vehicle applications. For this reason, it is critical that any evaluation of the fuel efficiency benefits that can be achieved when implementing advanced vehicle technologies must be based on a usage that is very representative of the fleet or application under consideration. For this project, a tractive energy analysis is used to estimate the potential for reducing fuel consumption when advanced efficiency technologies, including combinations of these technologies, are employed. The analysis uses relatively simple assumptions to generate a first order estimate of the fuel savings that can be realized when basic parameters of the vehicle configuration are changed, as occurs with the implementation of fuel efficiency technologies. The intention of this analysis approach is to identify the classes of technologies that have the greatest potential for improving fuel economy, and when the model is used with a drive cycle that is highly characteristic of the overall usage of a fleet or for a given application, the results provide a means to quantify the fuel savings that can be expected for that fleet or application when a particular technology or set of technologies is implemented. This can also serve as a starting point for performing cost evaluations to select technologies (and ultimately specific products) that can yield the greatest efficiency gains in the most cost-effective manner.

While a vehicle is driving, the power required at the wheels (referred to as the tractive power) is determined from the combination of all forces acting on the vehicle. The instantaneous power from the engine consists of the driving tractive power plus any power transmission losses in the drivetrain of the vehicle and the mechanical power consumption of the accessories that are driven by the engine, such as the air conditioning system, engine fan, alternator, etc. In most cases, and especially for trucks, the driving tractive power represents a dominant portion of the engine power, and the other power inputs can be represented with reasonable accuracy simply by using an average value for the accessory power and a constant value for the drivetrain efficiency. If more precise information is available and it is 
relevant in evaluating a particular technology, more detailed data regarding the power consumed by individual accessories can be included in the model, but in most cases the tractive contributions to the overall power requirements are what one wishes to evaluate.

It should be understood that most fuel efficiency technologies operate by reducing various energy losses that are inherent in the vehicle operation. For example, aerodynamic drag reduction devices such as fairings, trailer skirts etc. provide a more streamlined vehicle profile, thereby reducing the drag forces that oppose the vehicle's forward motion. The drag reduction causes the driving tractive power requirement to be lessened, and fuel consumption would thus be reduced for the same drive cycle. Not all periods or types of driving provide the same benefits from a technology, however, and in effect, each technology functions by reducing energy losses over a specific portion of the drive cycle. In the case of aerodynamic drag, for example, higher speeds generate greater savings for a given drag coefficient improvement, and there are no tractive energy savings impacting the fuel consumption for a conventional vehicle (without regenerative braking) during the periods of operation when the vehicle is decelerating. This is because engine power output, beyond engine idling, is only required during periods of positive tractive power, and during braking the only effect that a reduction in the aerodynamic drag causes is that a greater braking force is needed to provide the same rate of deceleration. For the drive cycle shown in Fig. 1, therefore, a reduction in aerodynamic drag will generate a reduction in fuel consumption only for those periods shown in black. Figure 2 shows the power associated with the aerodynamic drag over the same periods for this drive cycle. The integral of this power gives the total aerodynamic drag contribution to the driving tractive energy requirement for the drive cycle.

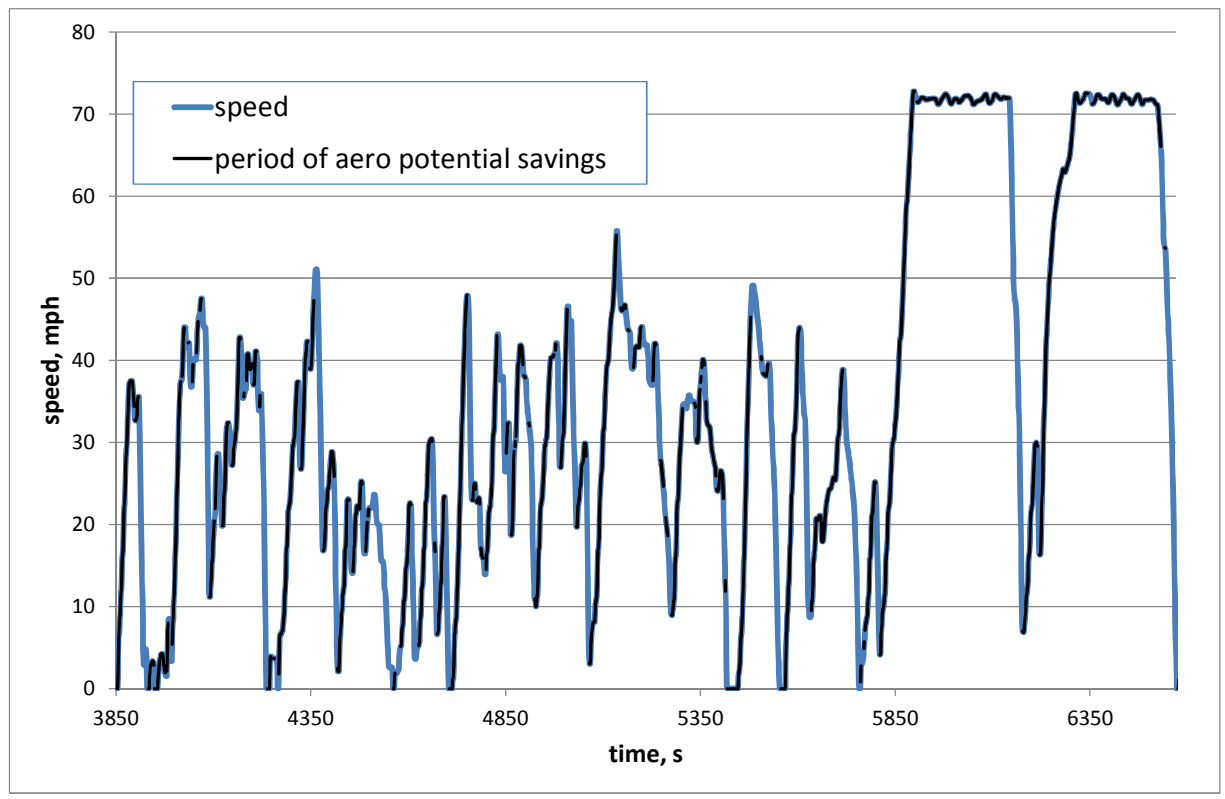

Figure 1 Drive cycle example to indicate the periods of tractive energy savings (in black) when aerodynamic drag is reduced for a conventional vehicle. 


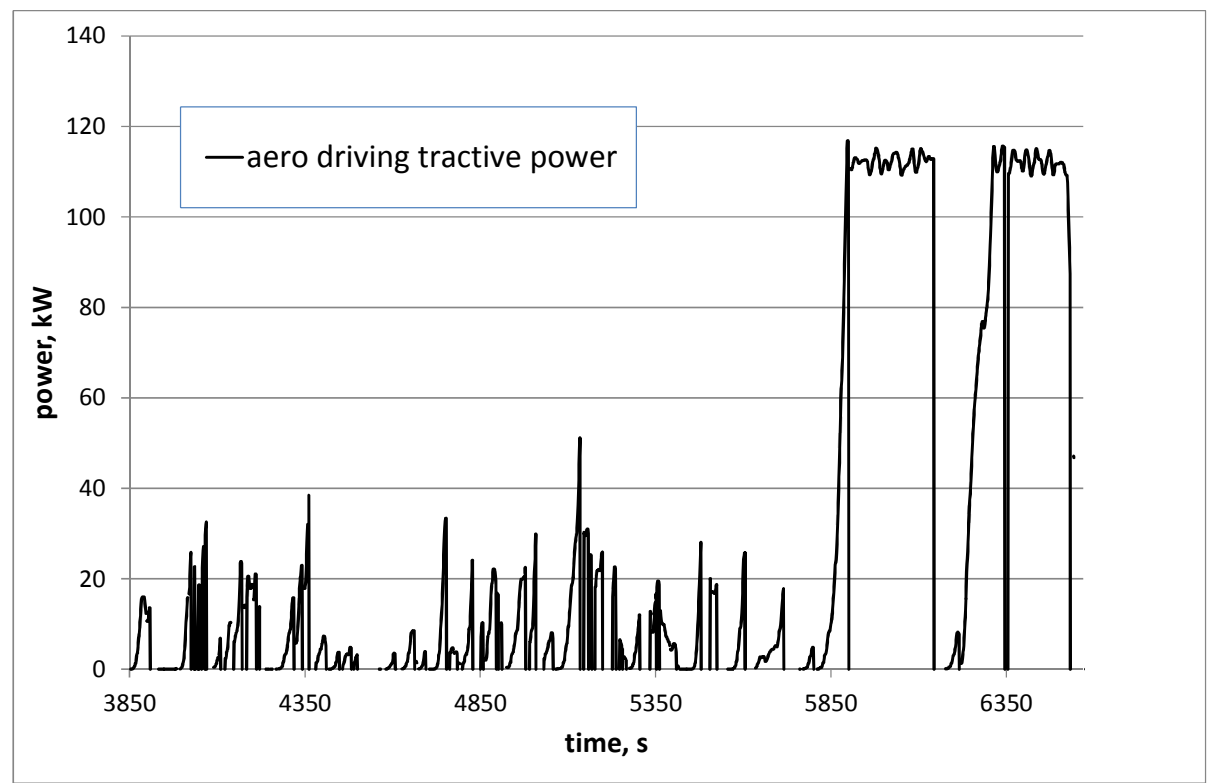

Figure 2 The aerodynamic drag power during periods of positive tractive power for the drive cycle shown in Figure 1. The integral of this power represents the aerodynamic drag contribution to the total driving tractive power.

If a hybrid system with regenerative braking is employed on a vehicle, then some portion of the energy that would be dissipated by the brakes in a conventional vehicle is recovered and stored by the regenerative braking system, and this stored energy will be used at a later portion of the drive cycle to reduce the tractive power required from the engine. This reduces the total driving tractive energy required from the engine, thereby reducing the fuel consumption for the drive cycle. In this case, we see that tractive energy savings are generated during the periods of braking as opposed to periods of driving tractive power for the regenerative braking system. The portion of the drive cycle that regenerative braking functions over is thus complementary to that of the aerodynamic drag (or tire rolling resistance) for a conventional vehicle. Figure 3 shows the portion of the drive cycle in which regenerative braking acts to recover tractive energy, and Fig. 4 shows the power associated with these braking periods, which represents the total available energy that can be recovered by the regenerative braking system. 


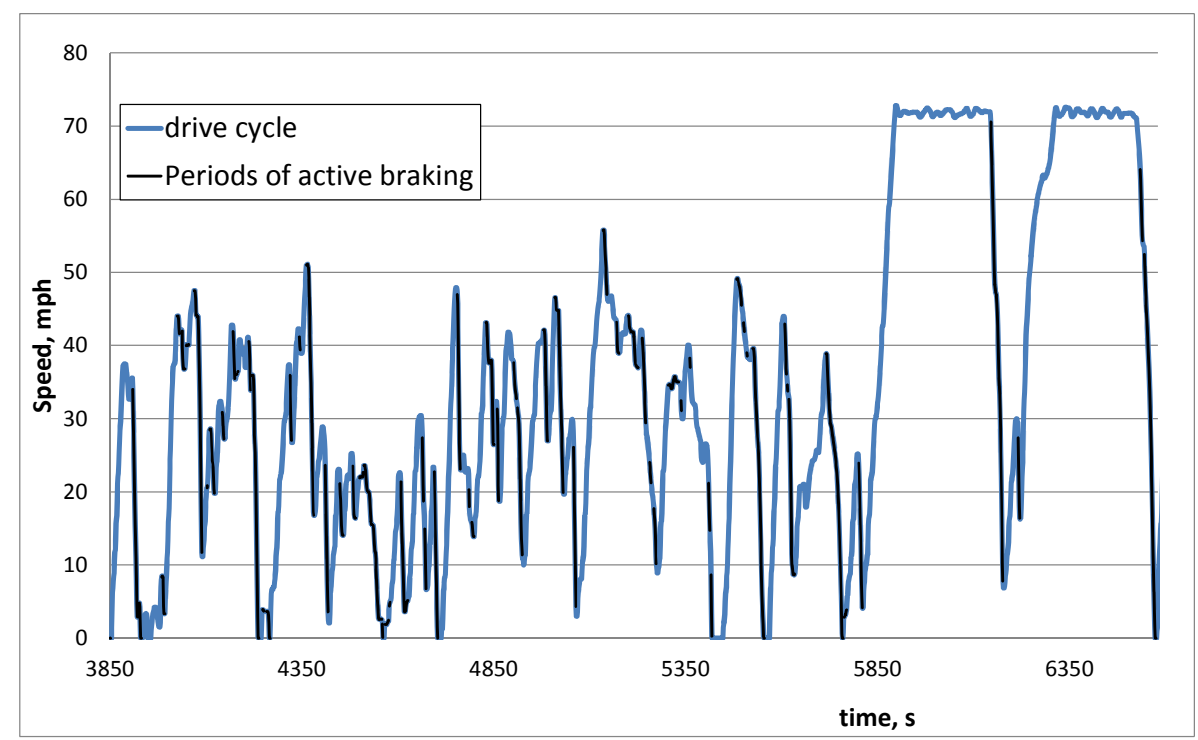

Figure 3 Periods of active braking during the drive cycle.

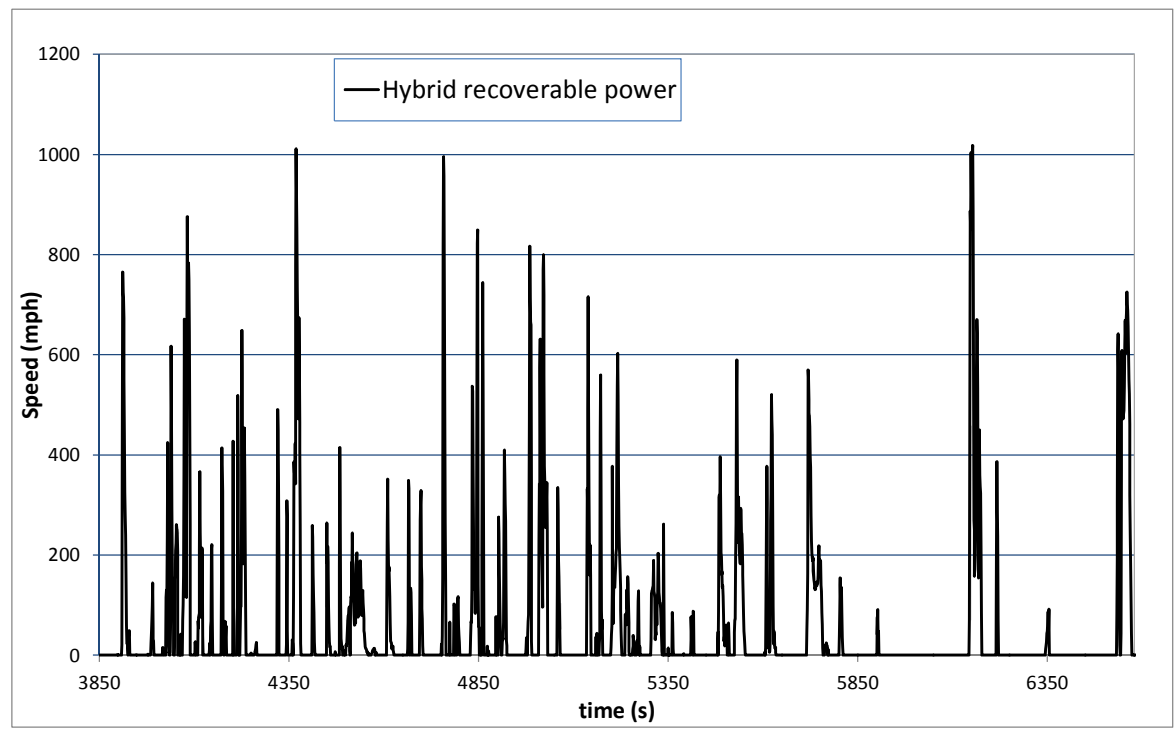

Figure 4 The braking power, which represents the maximum recoverable power with regenerative braking.

If regenerative braking is used in combination with rolling resistance or aerodynamic drag reductions, then more power is available for the regenerative braking recovery during the braking portions of the drive cycle than would be present without the aerodynamic or rolling resistance improvement. By properly tracking each of the energy losses over the entire drive cycle and accounting for the power from each energy loss term during periods of both positive (driving) tractive power and negative (braking) tractive power, the contributions from each energy loss factor to the total mechanical energy required from the engine can be quantified in detail. When the total contribution of each loss factor to the total driving tractive energy is known, it is straightforward to quantify the savings that can be generated if a technology is used that reduces that energy loss factor. 
The instantaneous power associated with each loss factor is calculated based on the physics associated with the corresponding energy loss that takes place. For example, the power associated with aerodynamic drag is calculated from the coefficient of $\operatorname{drag} C_{d}$, the vehicle frontal area $A_{f}$, and the speed $v$ using the relationship $\frac{1}{2} \rho v^{3}\left(C_{D} A_{f}\right)$, where $\rho$ is the air density. To distinguish between the periods of driving and braking tractive power, one needs to only consider if the net force at the wheels is positive or negative. The tractive power requirement is positive (driving) if

$$
\frac{d v}{d t}>-g \sin \theta-\frac{F_{a e r o}+F_{R R}}{m}
$$

and is negative (braking) if the inequality is reversed. Equality occurs when the vehicle is coasting, so the comparison shows whether the current acceleration is greater or less than the instantaneous coasting acceleration, which can be positive, negative or zero depending on the magnitudes of the aerodynamic and rolling resistance forces, $F_{\text {aero }}$ and $F_{R R}$, respectively, and the grade (given by $\sin \theta$, where $\theta$ is the angle relative to horizontal of the roadway). Note that $g$ appearing in Eq. (1) is the gravitational constant and $m$ is the mass of the vehicle. When the vehicle coasts, the tractive energy does not change even though the energy associated with the rolling resistance and aerodynamic drag continue to accumulate. During these periods, the combined potential and kinetic energies will decrease by the same amount as the energy losses from the rolling resistance and aerodynamic drag. To simplify the accounting of the energies, it is simplest to consider the periods of coasting as contributing to the driving tractive energy. This approach was followed for the tractive energy analysis, although there is very little impact on the results since it is very rare to have strict equality when performing calculations with numerical data.

The net tractive energy change for any period of driving includes the dissipative energy losses from rolling resistance and aerodynamic drag as well as the net change in kinetic and potential energies experienced. The result is

$$
\begin{aligned}
\Delta E_{\text {trac }} & =\frac{1}{2} m\left(v_{2}^{2}-\mathrm{v}_{1}^{2}\right)+m g\left(h_{2}-h_{1}\right)+\Delta E_{\text {aero }}+\Delta E_{R R} \\
& =\Delta E_{\text {kinetic }}+\Delta E_{\text {potential }}+\Delta E_{\text {aero }}+\Delta E_{R R}
\end{aligned}
$$

The driving tractive energy over the complete drive cycle, which is closely related to the mechanical energy output required from the engine, is therefore obtained by summing the tractive energy changes over all of the segments in which the tractive power is driving:

$$
E_{\text {trac }, \text { drive }}=\left[\frac{1}{2} m \sum_{i, \text { non-braking }}\left(v_{e, i}^{2}-v_{s, i}^{2}\right)+m g \sum_{i, \text { non-braking }}\left(h_{e, i}-h_{s, i}\right)+E_{\text {aero,drive }}+E_{R R, \text { drive }}\right]
$$

where $v_{s, i}$ and $v_{e, i}$ are the speeds at the start and end of each time segment $i$ for which the tractive power is driving, and $\mathrm{h}_{s, i}$ and $h_{e, i}$ are the elevations at the start and end of each driving tractive power segment. The summations are performed over all of the segments where the tractive power is nonnegative.

The contribution to the driving tractive energy from the tire rolling resistance is given by 


$$
E_{R R, \text { drive }}=\int_{t_{\text {drive }}} P_{R R} d t=C_{R R} m g \sum_{\text {non-braking }} \Delta s_{i}
$$

where the integration of the power associated with the rolling resistance is performed over the periods when the tractive power is non-negative (Eq. (1)). This result uses the basic linear relationship that the rolling resistance force, $C_{R R} m g$, is proportional to the load carried by the tires, where an average coefficient of rolling resistance, $C_{R R}$, is used to characterize all of the tires on the vehicle. The summation on the right hand side of the equation is the total distance traveled during the periods of driving tractive power. The rolling resistance contribution to the braking tractive energy is given using the same equation, but with the integration performed over the periods when the tractive power is negative. The result is simply

$$
E_{R R, \text { braking }}=C_{R R} m g \sum_{\text {braking }} \Delta s_{i}
$$

The aerodynamic drag contribution to the driving tractive energy is given by

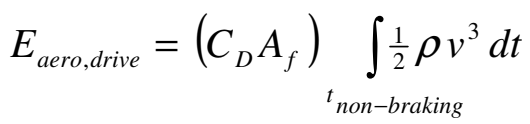

and as in the case for the rolling resistance, the braking tractive energy contribution from aerodynamic drag is determined simply by integrating over the periods of braking tractive power as opposed to the non-braking periods:

$$
E_{\text {aero,braking }}=\left(C_{D} A_{f}\right) \int_{t_{\text {braking }}} \frac{1}{2} \rho v^{3} d t
$$

The force required for braking (taken as a positive value) is equal to the absolute value of the tractive force during the periods of braking tractive force, and is obtained from a basic consideration of the forces acting on the vehicle,

$$
F_{\text {brakes }}=-\left(m \frac{d v}{d t}+m g \sin \theta+F_{\text {aero }}+F_{R R}\right) .
$$

The braking energy over the drive cycle is calculated by integrating the braking power, and the final result is given by

$$
E_{\text {brakes }}=-\left[\frac{1}{2} m \sum_{i, \text { braking }}\left(v_{e, i}^{2}-v_{s, i}^{2}\right)+m g \sum_{i, \text { braking }}\left(h_{e, i}-h_{s, i}\right)+E_{\text {aero,braking }}+E_{R R, \text { braking }}\right] .
$$

The summations are performed over all of the segments where the tractive power is negative. The energy consumed by the brakes is not included explicitly in the driving tractive energy, Eq. (3), but vehicle braking acts as a dissipative force, in a manner similar to rolling resistance and aerodynamic drag, that results in additional tractive energy being required to travel the distance traversed over the drive cycle. Dissipative braking actually increases the energy required from the engine since subsequent 
acceleration is necessary to attain the speed prior to braking (on flat ground at least) and the braking energy therefore, paradoxically, contributes to the driving tractive energy. It is easily shown using Eqs. (3) through (7) that the total driving tractive energy can be expressed as the sum of the driving and braking contributions of aerodynamic drag and rolling resistance plus the braking energy:

$$
E_{\text {trac,drive }}=E_{R R, \text { drive }}+E_{R R, \text { braking }}+E_{\text {aero,drive }}+E_{\text {aero,braking }}+E_{\text {brakes }} .
$$

Regenerative braking allows the energy that would otherwise be dissipated to be converted to another form of energy and stored so that it can be used at a later time during the drive cycle. To account for regenerative braking, it is assumed that some fraction of the braking energy could be recovered with a hybrid system, and this fraction is subtracted from the braking losses. $(80 \%$ is assumed in the results presented for this study, since this is a reasonable round-trip energy efficiency for the conversion from mechanical to electrical energy and back for a generator, battery and motor system. The tractive energy analysis aims to quantify the total energy savings potential when implementing various technologies, and this value is probably representative of an upper limit that might be achievable with any real hybrid system. In any event, it is acknowledged that this approach is rather simplistic, but the actual braking energy recoverable with a hybrid system depends on the size of the motor/generator and the battery, in addition to the power levels dissipated during each deceleration experienced during the drive cycle. In other words, it is dependent on the specific hybrid system used as well as by the actual drive cycle. If the estimated energy savings potential from the tractive energy analysis justifies consideration of the use of a hybrid system for a particular application, then more in-depth analysis should be pursued to determine what characteristics of the system are needed to achieve acceptable results.

With the tractive energy calculated, the total mechanical work required from the engine during the tractive periods is calculated by assuming a constant value of the driveline transmission efficiency, $\eta_{\text {trans }}$, and a constant accessory power, $P_{\text {acces }}$. The engine work associated with the periods of driving tractive power output is therefore given by

$$
W_{\text {eng,trac }}=E_{\text {trac }, \text { drive }} / \eta_{\text {trans }}+E_{\text {acces }} .
$$

Finally, the conversion of the fuel to mechanical energy is assumed to be provided by an average engine thermal efficiency, $\eta_{\text {engine, }}$ and the heat of combustion, taken to be the lower heating value of the fuel, LHV, is used to determine the volume of fuel consumed. The result for the fuel consumption, as a function of the driving tractive energy is the following:

$$
m_{\text {fuel }}=\frac{E_{\text {fuel }}}{L H V}=\frac{1}{\eta_{\text {engine }} L H V}\left(\frac{E_{\text {trac }, \text { drive }}}{\eta_{\text {trans }}}+E_{\text {acces }}\right) .
$$

This equation, combined with the driving tractive energy calculation and the tractive energy savings associated with the implementation of technologies, can be used to quantify the fuel savings that is expected from any combination of the technologies. Eq. (10) can also be used to assess improvements in engine efficiency when the impact on the average value of $\eta_{\text {engine }}$ can be estimated, and reductions to the average accessory power requirement can also be evaluated directly and compared with the other technology assessments using this approach. Although this level of evaluation is rather superficial for 
evaluating an engine redesign, it does allow the general magnitude of improvements that can be expected for the many technology options and combinations that might be considered to be compared simultaneously. As a final technology that can be considered with the others discussed, idle reduction technologies, such as an auxiliary power unit (APU), can be quantified based on a knowledge of the difference in fuel consumption between the engine when it is idling and the fuel consumption rate of the APU. To evaluate this accurately, the average time fraction of idling for the fleet operation must be known, but this is effectively part of the determination of a characteristic drive cycle, which we address in section 3 of this report.

\subsection{Calculation of Energy Savings Due to Vehicle Efficiency Technologies}

Once the contributions from the rolling resistance, aerodynamic drag and braking to the tractive energy are calculated, the fuel savings that can be realized with specific technologies that reduce these energy losses can be calculated. The regenerative braking savings potential was discussed above, and different assumptions could certainly be used if particular systems have been characterized more accurately for the regeneration efficiency. Fortunately, the situation is easier for the other terms in the tractive energy calculation, although some knowledge of the magnitude of reduction for the rolling resistance coefficient and the aerodynamic drag coefficient are necessary. The equations used in the tractive energy model are simple but realistic for the rolling resistance and aerodynamic drag, and the estimates for the energy savings are expected to be relatively accurate if the coefficient values used in the model are reasonable. For tire rolling resistance, a common improvement that might be considered is to replace conventional dual tires with new generation wide base single (NGWBS) tires. With existing tire technologies, a typical reduction in the average rolling resistance coefficient for a tractor-trailer when the drive and trailer dual tires are replaced with high efficiency NGWBS tires would be in the range of 0.001 to 0.002 ( 1 to $2 \mathrm{~kg} /$ ton) relative to a typical starting value of 0.007 . A reduction of 0.0015 (1.5 $\mathrm{kg} /$ ton) for the rolling resistance coefficient is assumed in the results presented in this study. Depending on the initial dual tires being replaced and whether or not high efficiency steer tires will also replace less efficient steer tires, the reduction could be even higher. For a particular case in which specific tires are known, actual rolling resistance coefficient values can be obtained and the reduction in the average rolling resistance coefficient can be calculated fairly precisely. For the reduction in the aerodynamic drag coefficient from various technologies, while detailed characterizations are not readily available for each technology, some values in the literature [3] and the level of fuel savings experienced by users of aerodynamic reduction technologies indicate that coefficient reductions of up to $10 \%$ are not unreasonable when implementing devices that are available on the market today. An assumed reduction by $8 \%$ from a nominal value for the aerodynamic drag coefficient of 0.62 is assumed for the results presented in this study.

Since the contribution to the driving tractive energy from the rolling resistance or aerodynamic drag is directly proportional to its corresponding coefficient $C_{R R}$ or $C_{D}$, respectively, the tractive energy change associated with the technology implementation is also proportional to the corresponding reduction in each of the coefficients. The tractive energy reductions from each factor are calculated individually and the total savings are subtracted from the nominal calculated value of the tractive energy. This approach allows the tractive energy savings to be quantified as a percentage of the total required tractive energy, 
which is a very natural and informative way to interpret the energy savings potential. Knowing for example, that the tire rolling resistance can provide, say, an $8 \%$ reduction in the total tractive energy establishes the benefits of replacing the tires as being significant and worthy of an evaluation of the cost effectiveness.

The tractive energy calculations can be performed using a single set of parameters representing a baseline vehicle configuration to determine the relative contribution from each energy loss factor, and these can be used in turn to quantify the change in the total tractive energy requirement that will be realized if any of the parameters for the tractive energy factors change. Once the tractive energy calculation is complete, a single calculation that accounts for driveline inefficiencies, the engine thermal efficiency and accessory power requirements can be used to estimate the overall impact on fuel consumption when any combination of the parameters that account for the technologies employed are changed. Finally, the fuel savings from idle reduction technology can be evaluated quite simply based on the difference between the baseline vehicle's idling fuel consumption rate and the rate of consumption when the idle reduction technology is employed (e.g. an auxiliary power unit). This simple approach using the tractive energy method allows fuel savings estimates to be made for any combination of the relevant technologies the savings can be evaluated simultaneously without the need to rerun a model multiple times. It also provides a direct, physics-based evaluation of the fuel savings potential for each technology, which can be used to select technologies that will be most cost effective for providing improved fuel economy.

The simplicity of this approach is a significant strength, but there are of course limitations to what can be achieved. In reality, the efficiency of an engine changes with the engine speed and torque. For the tractive energy model, a single average thermal efficiency value is used to determine the fuel energy required for the engine to generate the tractive energy over the complete drive cycle. Changes to gearing and detailed engine design changes that impact engine efficiency over a range of specific operating conditions cannot be accounted for with this approach and a more precise modeling methodology (such as a vehicle performance simulation using software such as Autonomie or GT Drive) would be necessary to obtain reasonable predictions of the effect of such changes. Nonetheless, even for two vehicles with different engines that are designed to operate in the same application, the space of engine operating conditions (with respect to torque and engine speed) that would be experienced could be expected to be relatively similar when operating over the same drive cycle. This being the case, the general trend for the efficiency maps will likely not differ dramatically, and differences in fuel consumption could still be adequately evaluated using this model based on a characterization of the average thermal efficiency for each engine.

\subsection{Validation of the Tractive Energy Method and Concluding Remarks Concerning its Relevance}

We now provide a final summary of the utility and capabilities of the tractive energy method to provide additional context. Based on an analysis of a characteristic drive cycle that is highly representative of the operations of a fleet or trucking application, we are able, using the equations presented in this section, to compare the fuel savings potential from the following technologies, or from any combination 
of these technologies: aerodynamic drag reduction, rolling resistance reduction, regenerative braking, mass reduction, engine efficiency improvements, reduction of the power from the vehicle accessories, and idling reduction. While the precision of the fuel savings estimates using only this model does differ among the different technologies, as noted in discussions above, the results from this assessment can be very instructive to identify those technologies that can yield the greatest reduction in fuel consumption. It should be emphasized that the tractive energy method relies strongly on a drive cycle evaluation, and in order to obtain the most meaningful results, it is important that the drive cycle evaluated be relevant to the application or fleet under consideration.

Now that we have presented the equations for the tractive energy calculation, a bit of validation concerning the accuracy of the method and its appropriateness for estimating fuel savings potential seems appropriate. It is first noted that estimating the engine power based on the tractive power requirement calculated from the drive cycle is quite accurate, even with the relatively simple assumptions used in the model. Figure 5 compares the measured power (the product of engine torque and engine speed) during a drive cycle plotted with the driving tractive power calculated using the tractive energy method and the estimated engine power, which is determined using Eq. (9). For this comparison, the instantaneous power values are evaluated, not just the total energy terms. While the equations for the power calculations were not presented here, they are quite straightforward to derive from the equations presented and the complete derivation is available in reference [2].

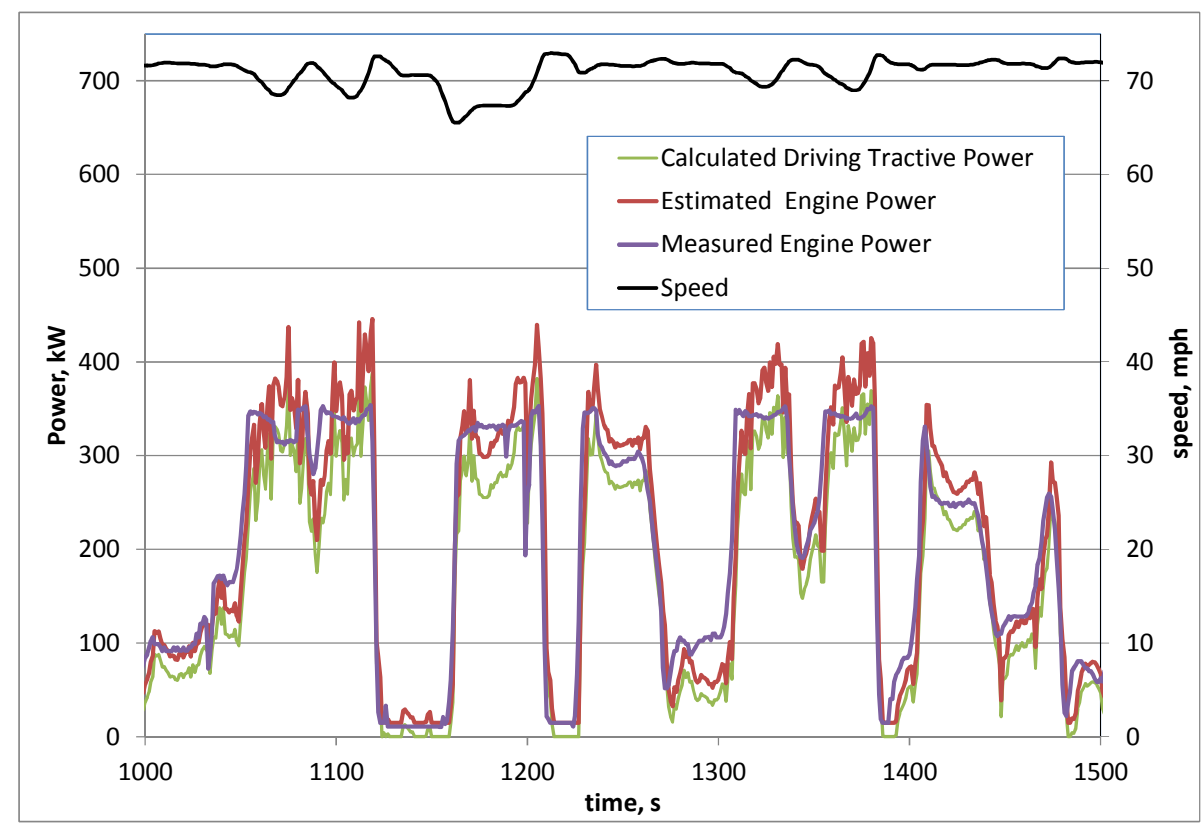

Figure 5 Comparison between the driving tractive power calculated using the tractive energy method, the engine power estimated from the tractive power, and the measured engine power during a measured drive cycle.

The tractive power by itself is reasonably close to the engine power, but by accounting for the transmission efficiency and a typical accessory power, the estimated engine power result is even closer to the measured engine power. It is evident from this comparison that the estimated engine power 
trends and overall magnitude are very well matched to the actual measured power from the truck's engine during the drive cycle. The parameters used in the evaluation were held constant in all cases for this study (except for the vehicle mass, which was determined separately for each segment of driving). Several different cases were evaluated to verify that the predicted power levels were reasonable, and the results prevented are quite typical, even without making any modifications to the parameters of the model for individual cases. This clearly shows that the driving tractive power (and the driving tractive energy, by extension) provides a very good representation for the actual engine power. It is noted that the six tractors tested in the HTDC project were "sister vehicle configurations" of the same type, with the only differences between them being the tires (NGWBS vs. dual tires) and manual vs. automatic transmissions. It is nonetheless quite impressive that the predicted engine power is so well represented with the simple model.

The measured cumulative fuel consumption is next compared to the driving tractive energy during the drive cycle. The two curves are shown separately in Fig. 6, and it is clear that the shapes of the two curves are quite similar during the drive cycle.

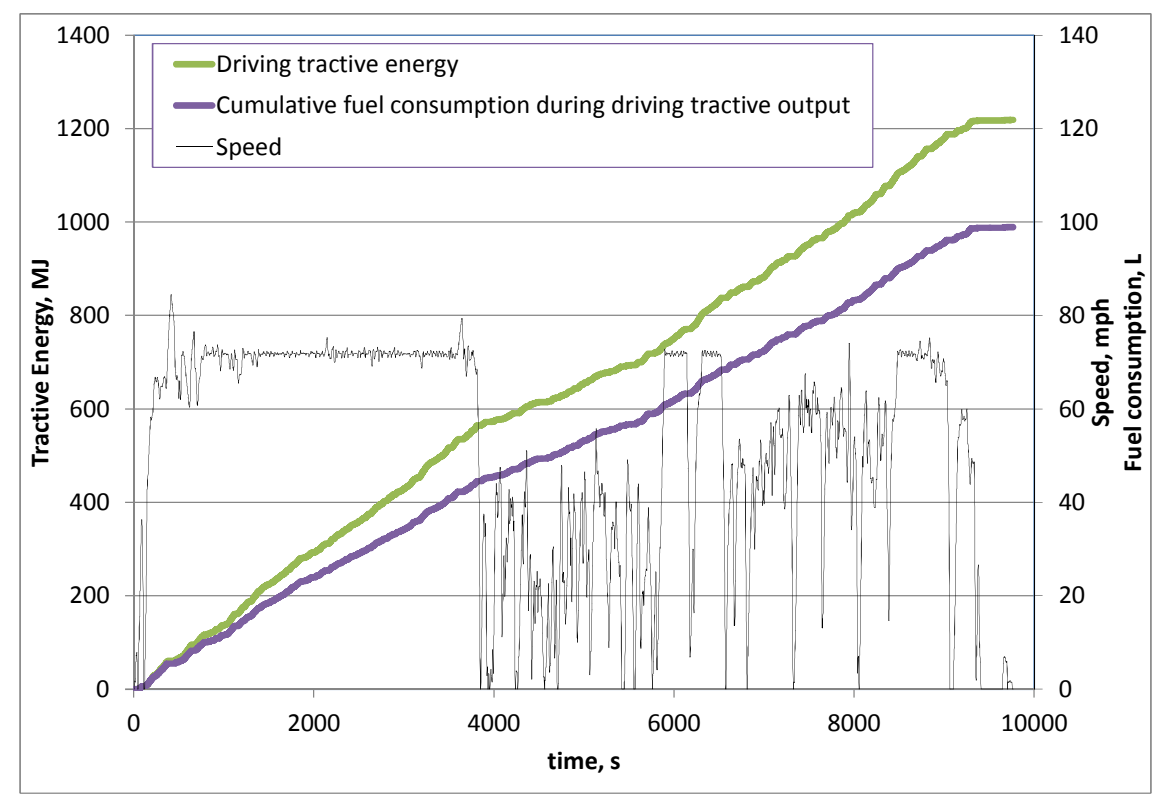

Figure 6 Comparison of the calculated tractive energy and the cumulative fuel consumption (measured) during periods of positive tractive power during a measured drive cycle.

In Fig. 7, we cross-plot the two curves, and it is clear that the correspondence is quite linear between the two variables, even with the very diverse driving conditions experienced during the drive cycle. This confirms that the tractive energy is indeed the primary factor responsible for the fuel consumption from the truck, and this one-to-one correspondence between tractive power and fuel consumption indicates that if the driving tractive energy is reduced (by means of a change in the vehicle configuration), then the fuel consumption will decrease in a linear manner. This result is a very strong validation of the tractive energy method. 


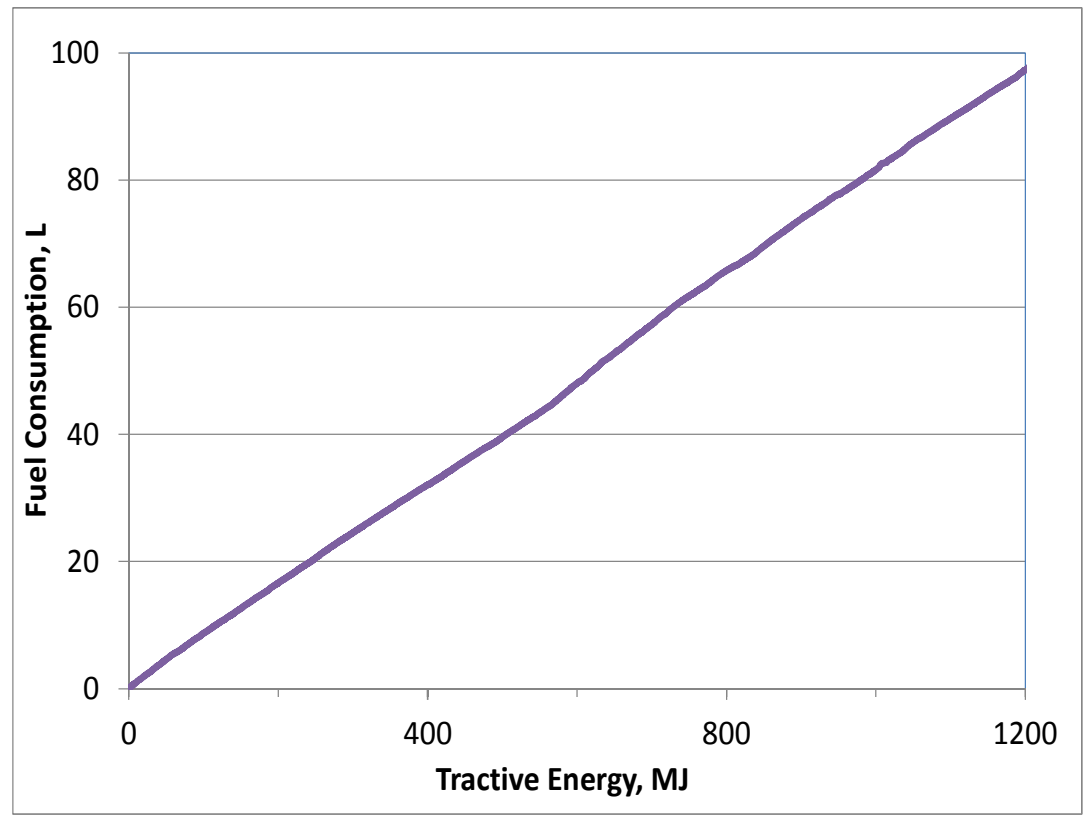

Figure 7 Cross-plot of the cumulative fuel consumption and the driving tractive energy results.

As a validation of the fuel savings predicted by the tractive energy model, ORNL has run several vehicle fuel consumption simulations using Autonomie. Autonomie is a plug-and-play vehicle simulation tool to assess fuel consumption based on a vehicle representation that includes specific engine map fuel consumption data, a complete specification of the drivetrain and a model to simulate driver shifting and acceleration performance [7]. It estimates fuel consumption by applying a detailed physical representation of all of the major powertrain components on the vehicle, and links the forces required to propel the vehicle with an engine map, which characterizes the fuel consumption of the engine as a function of engine speed and torque. An Autonomie vehicle model has been developed at ORNL to represent the vehicles used in the HTDC study. A heavy duty diesel engine map available in Autonomie was used in the model, but the engine parameters were tuned to be representative of the 15-liter, 6cylinder Cummins ISX 475 diesel engine that was present on the HTDC test vehicles. The transmission in the model is a 10-speed manual transmission, which is also representative of those tractors with a manual transmission used in the study.

For one of the drive cycles that has been analyzed in detail with the tractive energy approach, a complete Autonomie truck model was created that uses the same set of baseline vehicle parameters as the tractive energy model. The parameter variations used in the tractive energy analysis were repeated in the Autonomie simulations in order to validate the energy savings estimates of the tractive energy method against the more detailed model. Figure 8 shows a comparison of the engine power predicted by both models for a portion of the drive cycle, while Fig. 9 is a comparison of the cumulative fuel consumption for the entire cycle. The fuel consumption predicted with the tractive energy model shows some slight deviations from the Autonomie prediction during periods when the driving style changes significantly. This is a result of a more detailed thermal efficiency in the Autonomie model that varies with engine load, whereas the tractive energy model assumes a constant thermal efficiency. Nonetheless, the results from both models match extremely well. 


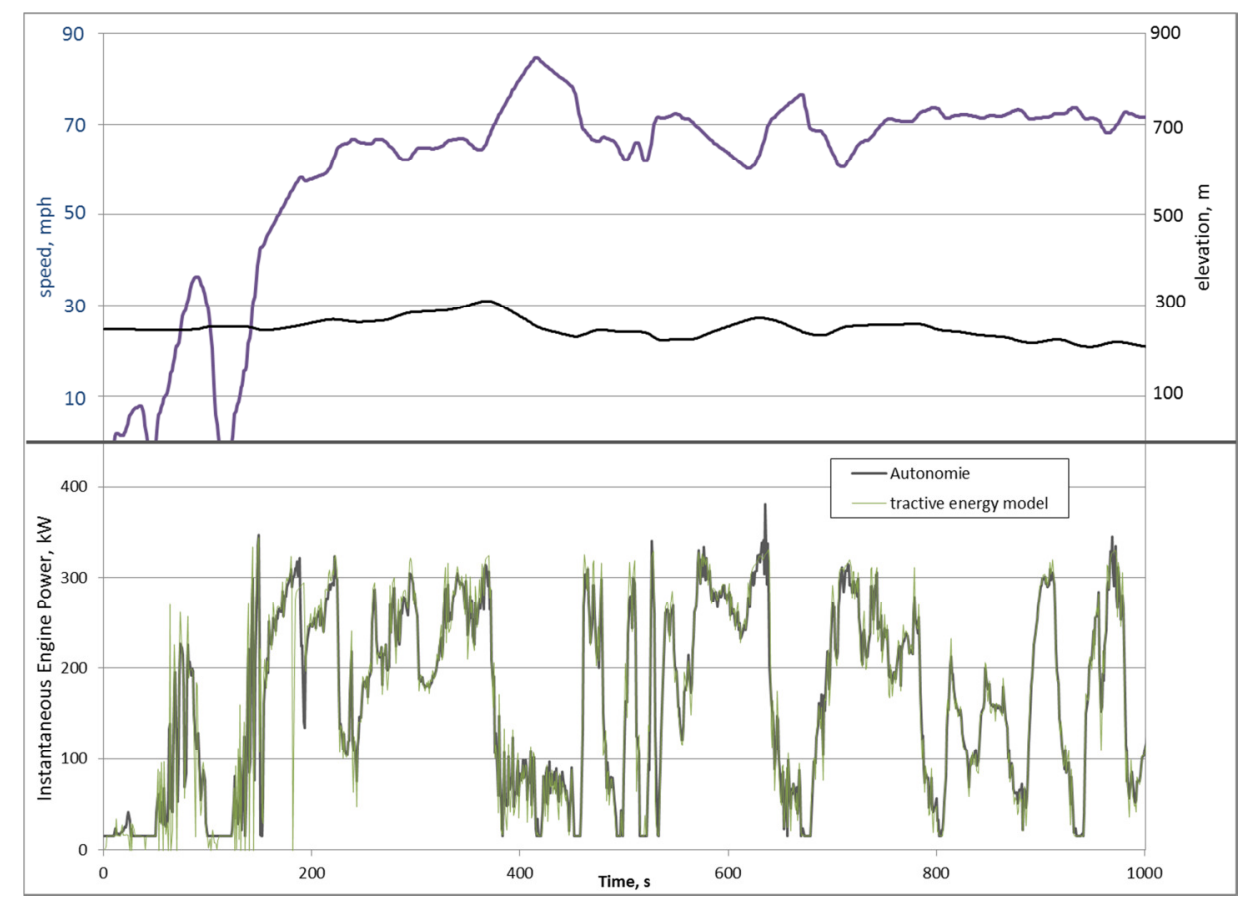

Figure 8 Comparison of the engine power requirement predicted with the tractive energy model and Autonomie for the first portion of the drive cycle.

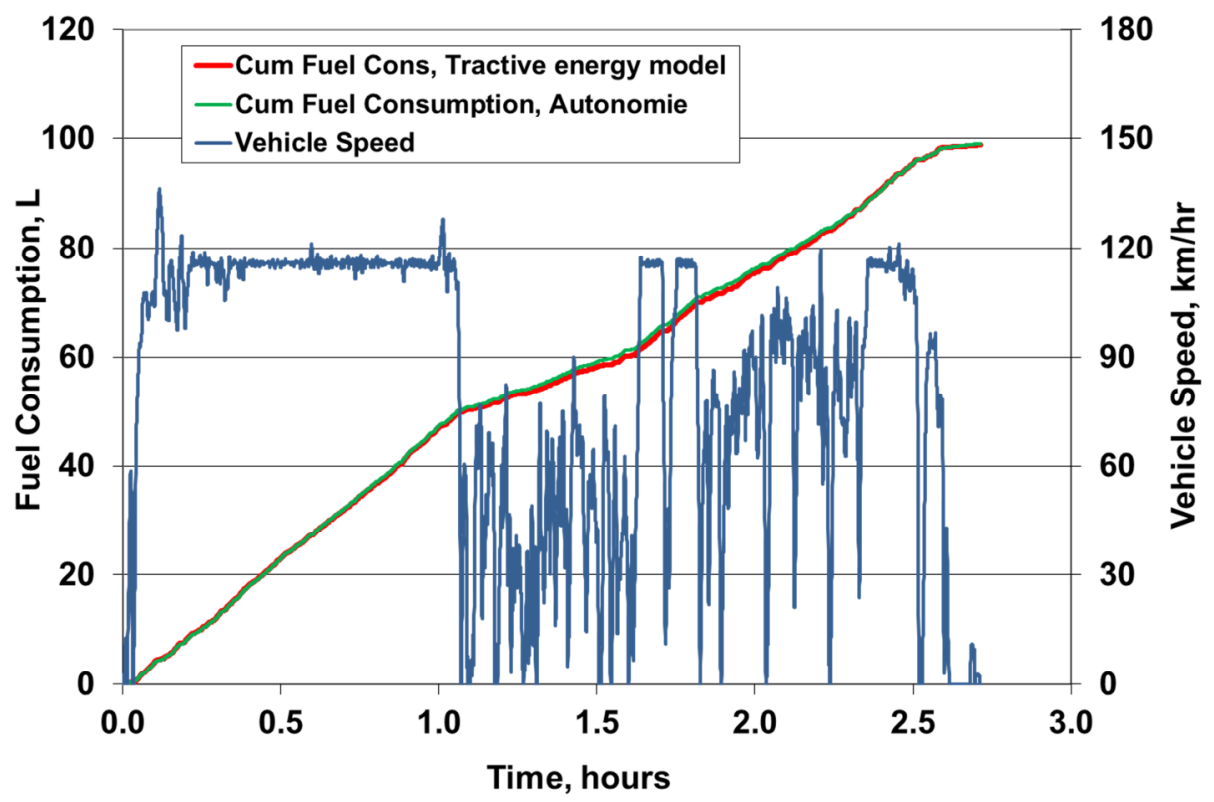

Figure 9 Comparison of fuel consumption predicted by the tractive energy model and by Autonomie.

Table 1 shows a comparison of the energy savings predicted by the Autonomie and Tractive Energy models. The first part of the table is the total energy required from the engine over the drive cycle, while the second part is the fuel consumption. Results are shown for several vehicle configurations, corresponding to parameter changes intended to represent the implementation of various vehicle efficiency technologies. 
Table 1: Comparison of results predicted with Autonomie to those from the tractive energy model.

\begin{tabular}{|l|cc|cc|}
\hline Cumulative Engine Energy Output (MJ) & \multicolumn{2}{|c|}{ Tractive Energy Model } & \multicolumn{2}{c|}{ Autonomie } \\
\hline Baseline* & 1462.18 & \% change from base & 1435.47 & $\%$ change from base \\
20T-Mass & 1431.96 & $-2.1 \%$ & 1407.28 & $-2.0 \%$ \\
$C_{R R}=0.0055$ & 1394.40 & $-4.6 \%$ & 1371.18 & $-4.5 \%$ \\
$C_{d}=0.52$ & 1392.29 & $-4.8 \%$ & 1368.32 & $-4.7 \%$ \\
$C_{R R}=0.0055 \& C_{d}=0.52$ & 1325.54 & $-9.3 \%$ & 1304.86 & $-9.1 \%$ \\
20T-Mass \& $C_{R R}=0.0055 \& C_{d}=0.52$ & 1297.59 & $-11.3 \%$ & 1278.49 & $-10.9 \%$ \\
\hline
\end{tabular}

\begin{tabular}{|l|cc|cc|}
\hline Fuel consumption (L/100km) & \multicolumn{2}{|c|}{ Tractive Energy Model } & \multicolumn{2}{|c|}{ Autonomie } \\
\hline Baseline* & 46.32 & $\%$ change & 44.94 & $\%$ change \\
20T-Mass & 45.38 & $-2.0 \%$ & 44.12 & $-1.8 \%$ \\
$C_{R R}=0.0055$ & 44.14 & $-4.7 \%$ & 43.08 & $-4.1 \%$ \\
$C_{d}=0.52$ & 44.07 & $-4.9 \%$ & 42.99 & $-4.3 \%$ \\
$C_{R R}=0.0055 \& C_{d}=0.52$ & 42.10 & $-9.1 \%$ & 41.15 & $-8.4 \%$ \\
20T-Mass \& $C_{R R}=0.0055 \& C_{d}=0.52$ & 41.15 & $-11.2 \%$ & 40.40 & $-10.1 \%$ \\
\hline
\end{tabular}

*Baseline configuration: $21,000 \mathrm{~kg}, \mathrm{CRR}=0.007, \mathrm{Cd}=0.58$

The tractive energy model predictions match those of the Autonomie model quite well in terms of the percent savings predicted from each technology. The differences in the engine energy output are within a small fraction of a percent between the two models for all of the parameter variations evaluated and the fuel economy prediction is also reasonable, although the tractive energy model was found to overestimate the benefits somewhat when using the default thermal efficiency value of 0.42 . The combination of cases provides a slightly higher error since the differences are cumulative. Nonetheless, this is a very good validation of the tractive energy model's ability to estimate fuel savings associated with advanced efficiency technologies. For this evaluation, the more complicated hybrid system was not considered since an accurate model of the hybrid powertrain for the heavy-duty truck would require a rather substantial effort, and this was not included in the scope of the present project.

\section{Preparation of Data Files from the Heavy Truck Duty Cycle (HTDC) Project}

This section describes the processing of data files from the Heavy Truck Duty Cycle (HTDC) project that were used to develop the characteristic drive cycle used for the analysis in this project. A brief summary of the HTDC project and data collection is also provided.

The primary data collection for the HTDC project [1] involved conducting a field operational test in which naturalistic driving data was collected and stored from six test vehicles. This testing was performed after initial development and proveout of a data acquisition system (DAS) and instrumentation that could withstand normal operation in a commercial vehicle over extended periods and would be able to operate with no human interaction for initialization on vehicle startup at the beginning of each day or at the beginning of each route. The data collection system consisted of four primary system components: 
the DAS, a vehicle self-weighing system, a weather station, and a GPS-based vehicle position and motion system. Measurements were also recorded from the vehicle's data bus, by direct connection from the DAS to the vehicle common area network (CAN). The resulting DAS was capable of collecting 60 channels of data and storing it internally in its dynamic memory. A working fleet (Schrader Trucking of Jefferson City, Tennessee) was selected for partnership in the project, and six tractors and ten box trailers (access was provided gratis by Schrader Trucking) were instrumented in the fall of 2006 using the HTDC DASs. The tractors were 2005 Volvo VNL sleeper cabs, equipped with Cummins ISX 475 engines. The fleet is a dry van truckload (TL) carrier with a regional usage. The first vehicle instrumented was closely monitored in the field for 30 days to evaluate and resolve any issues with the equipment, installation, driver distraction, and software prior to beginning the field operational test.

The drive cycle data collection for the HTDC project began in October 2006 and continued until November 2007. The six instrumented heavy trucks cumulatively hauled freight over more than 690,000 miles, primarily in the southeastern United States within a 500 miles radius of the fleet's base of operation in Jefferson City, Tennessee. During the field operational test, 60 channels of data were recorded at a rate of $5 \mathrm{~Hz}$ ( 5 samples for each data channel every second) any time that the engine was running on each test truck for a twelve-month period, resulting in the collection of $290 \mathrm{~GB}$ of raw data. The data collected during the field operational test is believed to be the most comprehensive real-world data set for Class-8 long-haul performance known to exist. The data collected during the FOT included speed, fuel consumption, road grade, location, and weather conditions.

After the drive cycle data collection was completed, one of the trucks tested in the HTDC project was delivered to West Virginia University (WVU), where it was emissions tested for several drive cycles on their Mobile Chassis Dynamometer and Mobile Emissions Measurement System (MEMS) [4]. Data from both the drive cycle measurements and the emissions testing were used for the current project.

\subsection{Data Processing}

This section documents the procedures followed in processing the measured drive cycle data prior to beginning the primary analysis. The data files were first cleaned, filtered and formatted for input into the drive cycle analysis tool.

A total of 1711 files, each representing one day of driving on an individual truck, were processed. The raw data from the HTDC project were available in text files with each channel stored in a separate column, and each row of data representing a separate time interval. The original data was measured every $0.2 \mathrm{~s}$, but it was decided for performing the drive cycle analysis to process the data at a lower frequency of $1 \mathrm{~Hz}$ since the velocity and elevation signals, which are most important for the drive cycle development, do not change substantially over time periods shorter than 1 second. Nonetheless, in preprocessing the files that served as inputs to the drive cycle analysis tool, the higher frequency data was retained in case there would be a need for any other purposes.

The channels that were included for performing the present analysis include the time, engine torque, elevation, engine speed, fuel rate, latitude, longitude, and speed. The measured fuel rate data and location information were not used in the main analysis, but they were included to permit additional 
evaluations in the event they would be needed. The engine speed and torque data were included to allow the measured power to be determined, since this data was necessary for calculating the vehicle mass, as described in the next section of the report.

The original HTDC data files were always divided at the start of a new day, that is at 12:00 a.m., if the vehicle was running at this time. If the speed was non-zero at the start or end of any file, i.e. if the truck was actually being driven at the day change, then the filtering of speed and elevation data for that file, which relies on forward and backward time calculations, would not provide the correct results. The drive cycle evaluation tool, used for the creation of the synthetic drive cycles, also requires each data file to start at zero speed. Therefore, the files from consecutive days were combined when a file ended with non-zero speed, and the division was made at the next stop. While combining the files, there were several checks of data integrity that were also performed, and information on the file length, maximum speed, average speed, average moving speed and any errors identified in the data for each of the files processed were recorded in a separate file. A sequence of Visual Basic for Applications (VBA) programs were written and run in the Excel environment to perform the file processing, and the files were cleaned and verified in a multi-step process while performing several quality checks of the data. Approximately 1000 lines of VB code were written to perform all of the data processing, filtering and verification functions. Files for which potential errors or corrupt data were identified were reviewed manually to determine what data could be recovered, and data that was clearly corrupt was removed. In some cases, one portion of the file would contain obvious errors, such as gaps in time when the speed was non-zero, speed values out of range or not reasonable based on prior or subsequent data, engine speed or torque data out of range or zero during driving, etc. Data was kept if it would not undermine the quality of the analysis, but in many cases, even large segments of corrupt or questionable data were removed from the files. Fortunately, the overall quality of the data was good, and only a small percentage of the original drive cycle data could not be used. Out of 1711 files, there were 24 files that were entirely or mostly corrupt, and 10 files for which more than 30 minutes of data was corrupt and removed. An additional 64 files contained short segments of data that were corrupt and removed or required cleansing. The primary cause of corrupt data was a lack of GPS data, which contained the elevation data channel (vehicle speed was still normally available from the vehicle CAN bus). Since the road grade, which is calculated from the elevation, is needed for the drive cycle development and the mass calculation procedure, the loss of the GPS signal for extended periods could not be recovered. In cases where one or only a few data points were corrupt in the middle of a long segment of driving, the unknown speed, torque or elevation data points were sometimes filled in by interpolation, or a short portion of the drive cycle data segment would be clipped from the data so that the previous non-corrupt data point matched the next valid speed point with the closest speed. For example, if corrupt data was present when the speed was at $105.5 \mathrm{~km} / \mathrm{hr}$., the corrupt data would be removed in addition to valid data until the speed returned to the same value of $105.5 \mathrm{~km} / \mathrm{hr}$. (or passed through this speed). Such modifications were only made if the corrupt data segments were relatively short within a long segment of continuous driving, so that the entire segment would not be lost due to a short occurrence of corrupt data. In any event, it is estimated that less than $3 \%$ of all of the raw data from the HTDC data set required removal or cleaning. This data processing nonetheless required a considerable amount of time 
to complete during the project, due to the quantity of data and the many details involved in performing the processing, but the data quality of the cleansed data is believed to be excellent.

The filtering of the speed data, which was performed prior to re-sampling the data at the $1 \mathrm{~Hz}$ frequency used in both the mass estimation and during the synthetic drive cycle analysis, used a discrete firstorder low-pass filter applied to a moving median of the raw speed signal. The moving median approach, which used points symmetrically from before and after each time being filtered, eliminates the effect of large deviations in the raw data from the overall trend, so individual data points exhibiting even large errors are rejected. By using a forward-rearward-based signal, the phase shift of the signal is eliminated so that this does not need to be accounted for separately in calculations of the tractive power or for comparisons with other data signals.

The elevation in the HTDC database was obtained from GPS measurements using a RaceLogic VBox II velocity and position measurement system. GPS elevation is known to be less accurate than horizontal positioning measurements (latitude and longitude) [5], and the accuracy of the elevation data recorded was an initial concern for being able to accurately quantify the tractive power requirements from the drive cycle data. The raw data for the GPS elevation was found to exhibit random jumps of up to a meter at any given time, and smaller variations over time in the GPS signal can cause the elevation data to drift somewhat, even when the vehicle is not in motion. Fortunately, roadway elevation does not change rapidly and is very smooth with respect to distance traveled. Engineering standards for road design [6] provide guidelines for how rapidly roadway grade should change, and the maximum grade is also limited based on the type of road traveled. Grades are limited to 7\% on interstate highways and $8.75 \%$ for the national highway system. This smoothness of road grade allows the time-based GPS elevation signal to be effectively filtered by smoothing the measured changes in elevation that occur as a function of distance traveled. To filter the signal, the sine value of grade (elevation change per unit distance traveled) was calculated using the speed signal in conjunction with the elevation. In a first pass, the grade was set to zero whenever the vehicle was not in motion, and if the calculated grade exceeded $8 \%$, it was assumed that the value was in error and the grade was set to the value of the previous time interval. This set an upper limit of $8 \%$ for the roadway grade, which was selected since the roads traveled by the trucks were primarily major highways where the grade should not exceed this value. A moving average of the grade was then calculated using forward and rearward data from the HTDC measurements, as in the case of the velocity filtering, so that there was no shift in the elevation data relative to the other data channels. The moving average provided a second degree of smoothing that gives a very clean set of grade data. The road elevation was then calculated by integrating the grade data using the distance traveled. The starting elevation from each file was used as the initial elevation for the filtered elevation data. It should be noted, however, that the tractive energy calculation does not depend on the absolute elevation, only the elevation changes.

The elevation determined using this filtering approach was compared with land elevation data extracted from the U.S. Geological Survey (USGS) map database (based on a lookup of the measured GPS coordinates) for several segments of travel to have some indication of the accuracy of the raw elevation data, and the grade data was then compared between the raw data and the filtered grade. Figure 10 shows a comparison of the raw GPS elevation data, the filtered data and the USGS survey data along a 
route traveled along Interstate 75 between Knoxville and Cleveland, Tennessee, in addition to some secondary roads in the Cleveland area. It is evident that the overall trend of elevation changes is correct for this data. The USGS database includes topological data points on a grid spaced at $30 \mathrm{~m}$ spatial resolution horizontally, and the elevation data was interpolated based on the input latitude and longitude values. This is therefore not an accurate representation of the roadway elevation data since the interpolated data does not correspond to the specific positional data in most cases, and the highway elevation will be considerably smoother than the surrounding landscape due to roadway design. The GPS measurement, in spite of its shortcomings, matches the overall elevation change extremely well. It should be noted that the filtered elevation data follows the raw data very closely for most of the segment traveled, to the extent that the two curves cannot generally be distinguished when considering the data over extended time periods. The signals can diverge somewhat at times, however, as in the last 1000 seconds of Fig. 10.

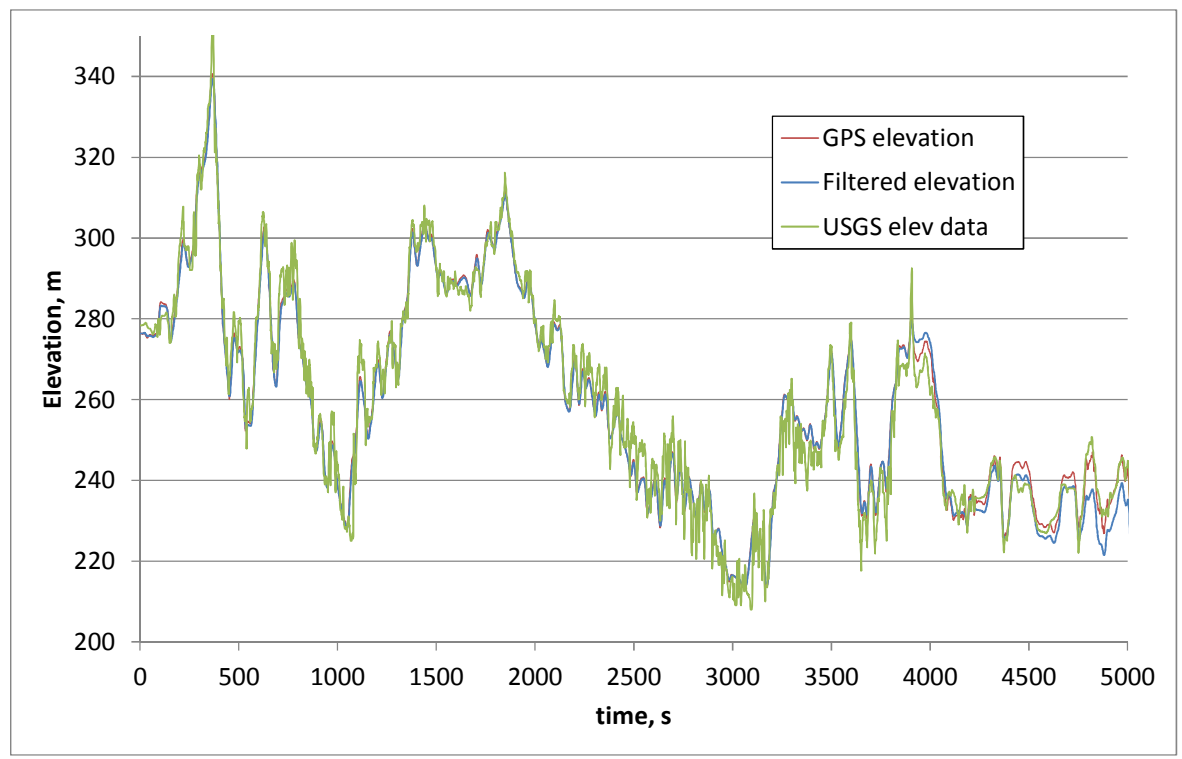

Figure 10 Comparison of the measured elevation and USGS elevation data for the same route (based on a lookup using the measured position).

Figure 11 shows a comparison between the grades calculated using the raw elevation data and the filtered grade. In the first segment, Fig. 11(a), the grade signal smoothing is rather subtle over most of the time traveled, but there are several apparent errors in the signal, with spikes that significantly exceed the $8 \%(0.08)$ levels permitted in the selected filtering methodology. For the segment shown in Fig. 11(b), the measured variations in grade are much more pronounced and the filtering is clearly necessary to remove some of the more frequent occurrences of spikes in the grade signal. 


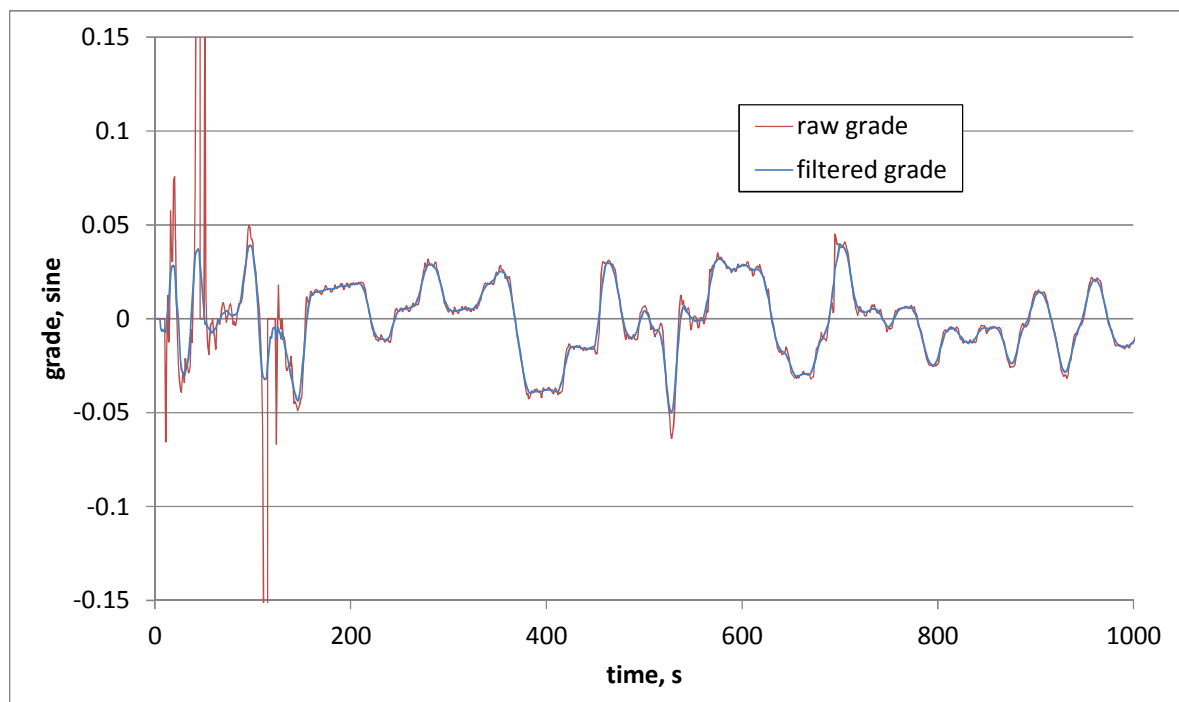

(a)

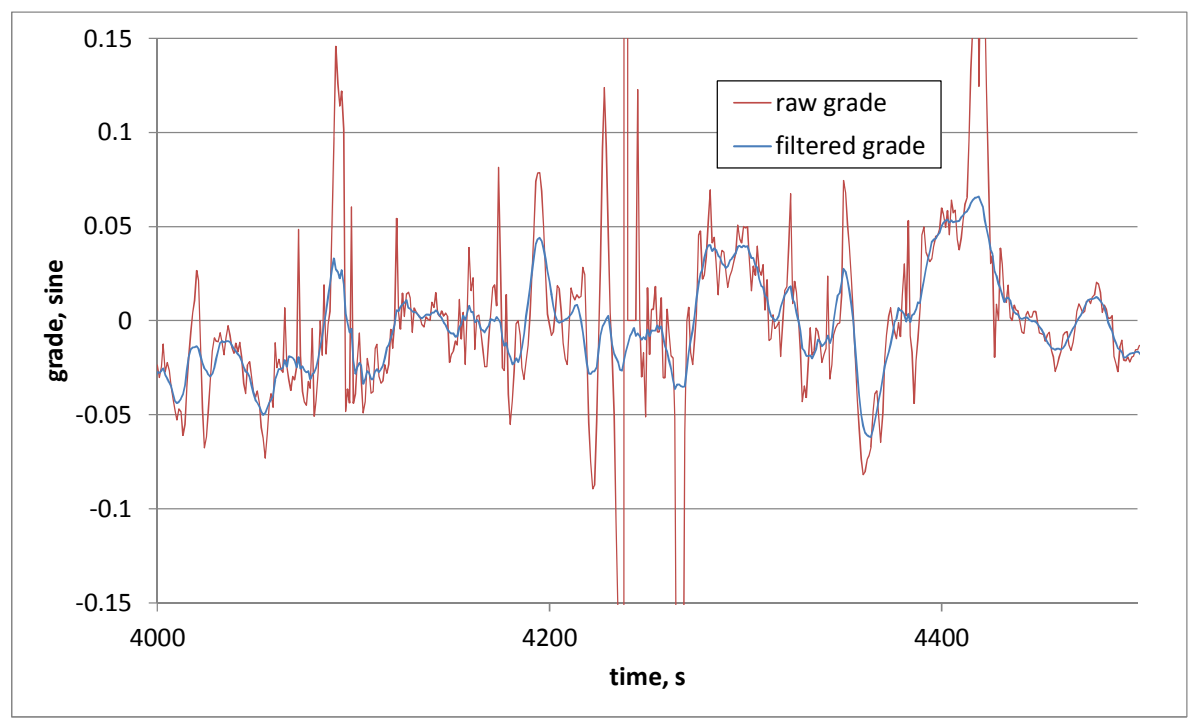

(b)

Figure 11 Comparison of the grade determined from raw elevation data and after filtering, for two time segments.

Although the elevation filtering approach used for the data processing does present the possibility of removing higher grade levels from the data, the measured elevation data includes enough noise that filtering is required. The approach selected provides a reasonable tradeoff that allows the general elevation changes to be captured while eliminating grade signals that, based on the nature of highway design, are unlikely.

\subsection{Mass Estimations}

Once pre-processing of all of the data files was completed, the project team was nearly ready to perform the drive cycle analysis to generate the synthetic drive cycles. The load carried by the vehicle, however, is also important for the drive cycle characterization and this data is needed for each "microtrip" in the drive cycle, i.e. each segment of driving between stops. (The term micro-trip is used in the literature for drive cycle evaluations, although we will not use it extensively in our discussion.) In 
developing the synthetic drive cycle, it was decided by the project team that separate evaluations should be performed for different ranges of the total vehicle mass so that large differences in acceleration capabilities would not be mixed. Additionally, since the mass plays a primary role in the level of tractive power that is calculated, separating the data based on several ranges of the mass allows more accurate evaluation of the complete range of tractive power variations that occur for the vehicles during actual use.

As mentioned previously in the report, ten trailers were instrumented (for measuring the mass of the trailer using an AirWeigh system), along with the 6 tractors. The fleet owned and operated over 180 trailers total, however, and normal operations did not permit the trailers instrumented for load measurement to always be paired with the instrumented tractors. As a result, in all but a small portion of the test data, the trailer attached to the tractor was not instrumented for load measurements, and the total tractor-trailer load measurement was thus not available for the majority of the data in the HTDC database. An approach was developed to infer the mass based on the other measured data from the vehicle. This section describes the mass estimation calculation and presents the results of the mass distribution for the fleet.

By using the engine torque data, a knowledge of the vehicle speed allows, in principle, a calculation of the mass based on Newton's second law of motion if we know all of the forces acting on the vehicle. Aerodynamic drag force, rolling resistance, and gravitational force can all be estimated using the same assumptions described in section 2 of this report. Even small errors in the measured speed data, however, can lead to large errors for instantaneous calculations of the acceleration, and the mass prediction done in this manner does not lead to consistent results during the driving segment. Averaging of the mass based on this approach is problematic, at least for an automated approach. If the measured engine power data is used to estimate the tractive energy, based on a reasonable assumption for power transmission losses and an average power required from the vehicle accessories, then the measured tractive energy over the drive segment (based on the measured engine power output) can be compared to the cumulative tractive energy that is calculated using only the drive cycle data (along with relevant vehicle parameters). By iteratively adjusting the mass value in the speed-based tractive energy calculation, one can determine the appropriate mass so that the two tractive energy values are matched. This approach integrates the instantaneous values of forces and accelerations so that the data are automatically smoothed and averaged using a physically based metric, the tractive energy, that relates directly and naturally to the vehicle mass.

The mass remains nearly constant during any period of continuous driving, since the only mass changebarring a loss of cargo while in transit-is the fuel consumed during travel, which is a very small fraction of the total vehicle mass. Mass changes due to cargo loading or unloading were only considered to take place at stops and it was assumed that the vehicle load change would not take place for stops less than 20 minutes in duration. These simple criteria allowed potential break points where mass changes might occur in each day's drive cycle to be identified automatically, and the mass estimation could proceed one segment at a time. The approach described in the previous paragraph was programmed using Visual Basic for Applications (VBA) in the Excel environment, and the mass estimation process was performed for each identified driving segment individually. If the mass in two or more sequential 
segments was found to remain within a specified tolerance, then the segments are joined and the average mass determined for the combined segments. This process is repeated by the program until the mass for each segment contained in each file is determined. The automated process allowed the mass calculations for all of the files to be performed efficiently. Once the mass calculation is complete for a file, each drive segment with a unique mass from the original drive cycle data file is exported as a separate drive cycle, and the mass, in kilograms, is appended to the filename so that the mass can be read by the tractive energy evaluation tool. The program plots both tractive energy curves along with the speed profile for each file after the mass is calculated and the image is exported to a .png file so that the results can be easily reviewed and verified. In some cases, a segment where a mass change took place was not identified by the tool and the tractive energy curves did not match over the full range. When this occurred, the mass calculation would be incorrect, so the file had to be reprocessed, with user intervention to control the divisions of the driving segments and adjustment of the mass values. Other problems with the mass calculation could occur, but by stopping the calculation process at particular points and manually adjusting the segment breakpoints prior to finalizing the mass recalculations for each segment, the correct segmentation could be achieved and the estimation of the mass was completed so that values for the two tractive energy functions (engine-power based and drive-cycle based) matched over the complete drive cycle.

Figure 12 shows a typical tractive energy plot when the automated mass calculation did not provide the proper segmentation. This type of error was easy to identify when reviewing the results, so it was clear which cases required user intervention to correct problems that occurred during the automated mass calculation. At about 8 hours into the day's drive cycle shown in the figure, it is clear that the mass changed (the predicted and measured tractive energy curves are not aligned) but the software did not identify the stop where the mass change occurred since there were two brief periods of movement that occurred during the time of loading. By rerunning the case with a breakpoint set in the code so that it stops after identifying the stops where mass changes are expected, the user can add the missing stop time to the list and restart the software to continue the calculation. Table 2 shows the data contained in the Excel calculator while the calculation is being performed. The mass program enters formulas into the spreadsheet to find the correct data for the table, and when a new stop point is entered into the main data sheet, the rest of the table is filled in automatically. This permits corrections to be done relatively easily when this type of problem occurs in the mass determination. To correct this particular issue, a manual override to set the end time to 8.0 (hours) for driving segment 7 was entered and the mass value from driving segment 7 was copied to the new driving segment 8 , before restarting the program to continue the calculation. With the two simple changes, it automatically recalculated the masses with the selected divisions and identified that mass case 6 and 7 were within tolerance for the same mass. The program combined these two driving segments by setting their masses equal in the spreadsheet before continuing with the calculation (as shown in the table, this was also the case for driving segments 1 through 5). The final result for the day's drive cycle was that three separate driving segments with different masses were identified, and the corresponding files with the drive cycle data were automatically written when the calculation was completed, and the image with the corrected tractive energy plots, as shown in Figure 13, was exported as a .png file. 


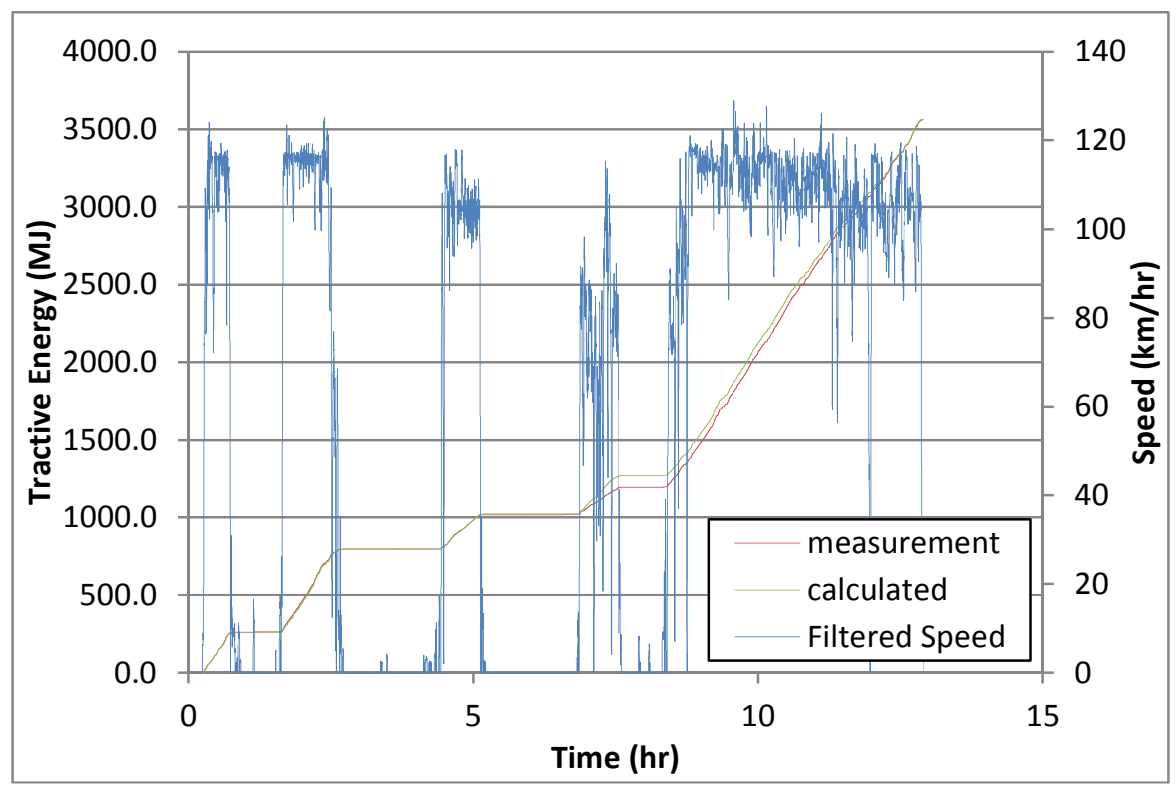

Figure 12 Plot of the tractive energy curves, based on measured engine data and determined from the drive cycle data, as used in the mass estimation. This sample case shows a mass change occurring at about 8 hours that was not properly identified by the automated calculation.

Table 2: Data contained in the Excel spreadsheet during the calculation of the mass for the case shown in Fig. 12.

\begin{tabular}{|l|l|l|l|l|l|}
\hline $\begin{array}{l}\text { Driving } \\
\text { segment }\end{array}$ & Mass, kg & End time, hr & $\begin{array}{l}\text { calculated } \\
\text { tractive } \\
\text { power, MJ }\end{array}$ & $\begin{array}{l}\text { measured } \\
\text { tractive } \\
\text { power, MJ }\end{array}$ & $\begin{array}{l}\text { end row } \\
\text { in data } \\
\text { sheet }\end{array}$ \\
\hline 1 & 27463.0 & 1.13 & 257.0 & 260.4 & 4077 \\
\hline 2 & 27463.0 & 1.52 & 257.7 & 261.2 & 5486 \\
\hline 3 & 27463.0 & 3.38 & 794.2 & 795.5 & 12158 \\
\hline 4 & 27463.0 & 4.13 & 794.8 & 795.5 & 14860 \\
\hline 5 & 27463.0 & 4.32 & 795.5 & 795.6 & 15543 \\
\hline 6 & 14720.3 & 6.82 & 1021.2 & 1019.3 & 24555 \\
\hline 7 & 25297.8 & 12.91 & 3566.7 & 3564.0 & 46476 \\
\hline
\end{tabular}




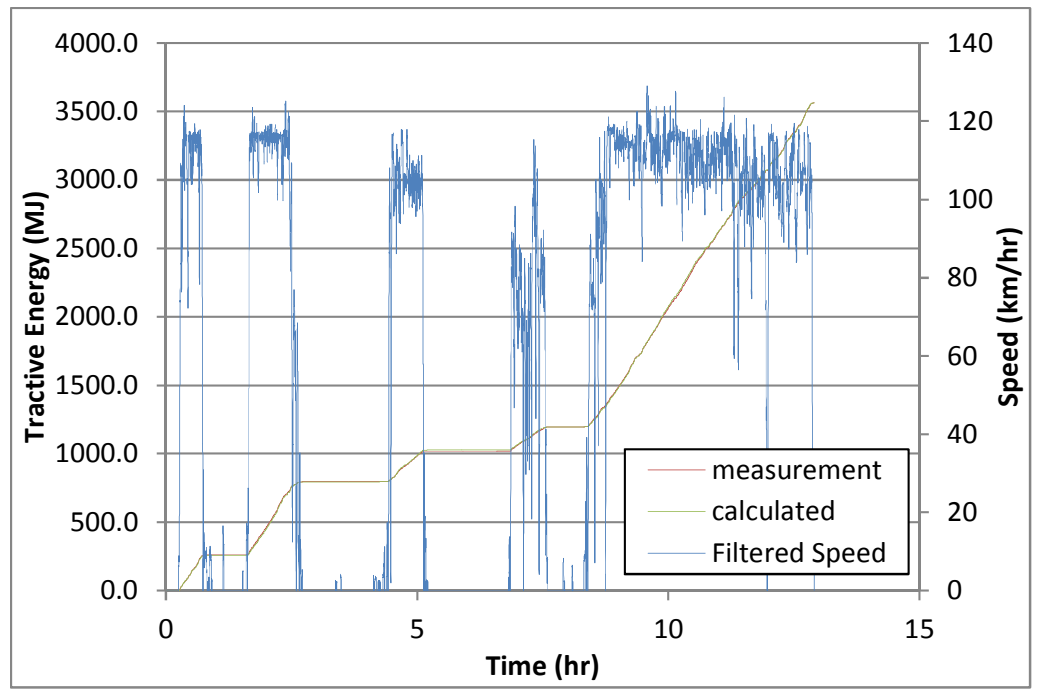

Figure 13 Plot of the tractive energy curves after the corrections were completed in the mass calculation.

The accuracy of the mass estimation was evaluated initially by comparing the calculated mass result with the measured mass for a case in which the trailer was instrumented with the AirWeigh measurement system. The AirWeigh system is designed to provide an accurate measurement when the vehicle is stopped, on level ground, and the parking brake is disengaged. Under these conditions, the measured weight should be within $1 \%$ of the actual vehicle weight. While driving, load transfer causes the indicated weight signal to vary about the actual weight. Figure 14 shows a plot of the measured mass value, determined by summing the AirWeigh mass data from the steer axle, drive axles and trailer axles, for one day's travel. Based on the figure, between 0 and 7.5 hours, the mass of the truck is approximately constant. During the stop following the first driving period, at about 2 hours, there is a minor increase in the mass that appears in the measured data. During this time, however, the GPS data shows that the truck was stopped at a rest area along the interstate highway, and it is quite unlikely that fuel was added or that the mass actually changed other than the driver exiting the vehicle. The variation in the signal during this period is likely due to the parking brake being applied and changes to the pressure in the air bags, which is what the system uses for measuring the weight. From the measured mass data from this signal, one sees that there is some uncertainty as to when the data is fully accurate. Nonetheless, the variation observed here is less than $700 \mathrm{~kg}$, and the uncertainty in interpreting the recorded signal is relatively insignificant relative to the mass data precision needed for our purposes. It should be noted that this uncertainty is not associated with any fault of the AirWeigh equipment, but rather is a consequence of the manner that it was used to record a continuous signal that we must interpret without the knowledge of air brake status and the local road grade during stops. In any event, the mass measurement from the AirWeigh system gives a signal that can be interpreted to get a reasonably accurate measurement of the mass during each segment of travel. 


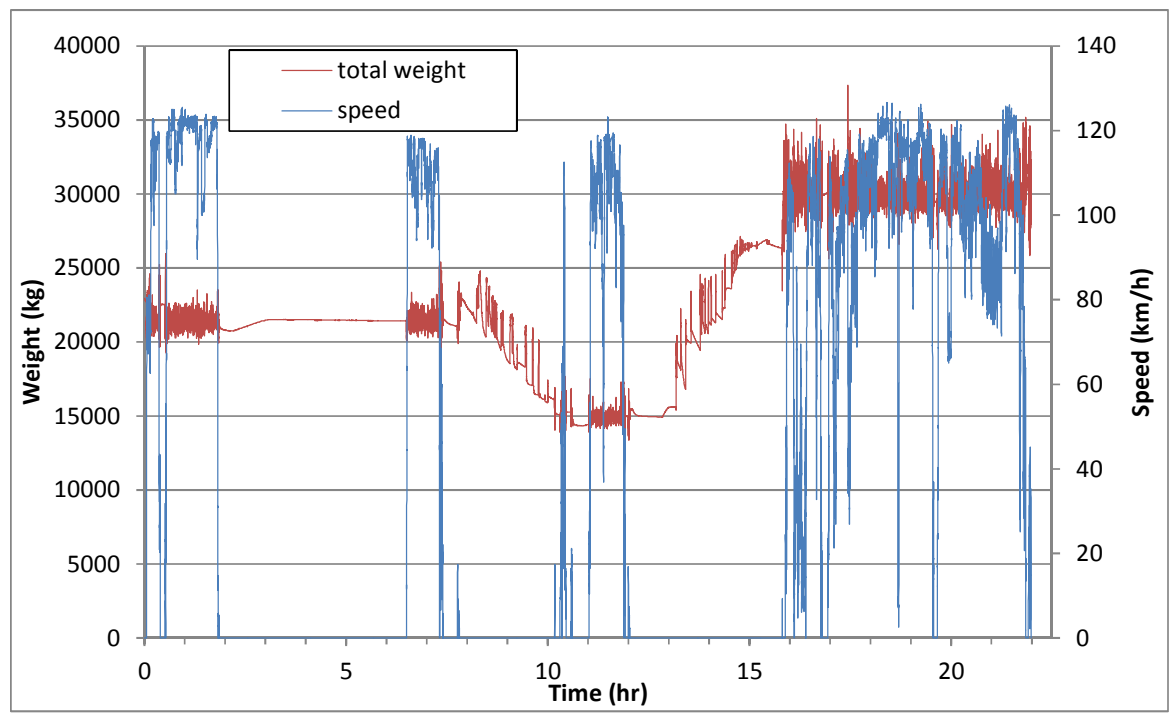

Figure 14 Plot showing the measured weight data during a day with two load changes.

The unloading of the trailer during the period from 8 to 10 hours can be observed in the mass plot, then after a period of travel between 10 and 12 hours, the trailer was reloaded to a third level of mass for the day. The measured values of mass for these three periods of different loads were $21,500 \mathrm{~kg}, 14,900 \mathrm{~kg}$, and $26,400 \mathrm{~kg}$, based on the mass before or after the load changes were complete. The corresponding masses predicted using the mass estimation algorithm for the three segments of travel were $21,650 \mathrm{~kg}$, $13,900 \mathrm{~kg}$, and $23,300 \mathrm{~kg}$, respectively. For the first two segments, the error between the calculated and measured mass was within $1000 \mathrm{~kg}$, and the error was about $3100 \mathrm{~kg}$ for the last segment. This level of accuracy, while not outstanding, was considered acceptable for the project, and the routine for the mass estimation was run to calculate the mass for all of the files.

As was the case for the day considered in the previous figure, it was rather common for the mass to change one or more times during a day's travel in the fleet tested. From the 1711 original files (one per truck for each day it operated), there were a total of 2918 mass-separated travel segments identified. The vehicle mass levels were categorized using three levels-low mass, medium mass and high massso that the driving segments from the separate ranges could be evaluated separately using the synthetic drive cycle tool. The low mass range was defined to correspond to a total vehicle mass below $20,000 \mathrm{~kg}$ $(44,000 \mathrm{lbs}$.); medium mass was defined by a vehicle mass greater than or equal to $20,000 \mathrm{~kg}$, but less than $28,180 \mathrm{~kg}(62,000 \mathrm{lbs}$.$) ; and high mass was defined to include all masses greater than or equal to$ $28,180 \mathrm{~kg}$. The distance traveled during each mass segment was calculated and the mass distribution as a function of distance traveled was determined. Figure 15 shows the mass histogram, and the separation points between the low, medium and high mass categories are shown with black dashed lines. 


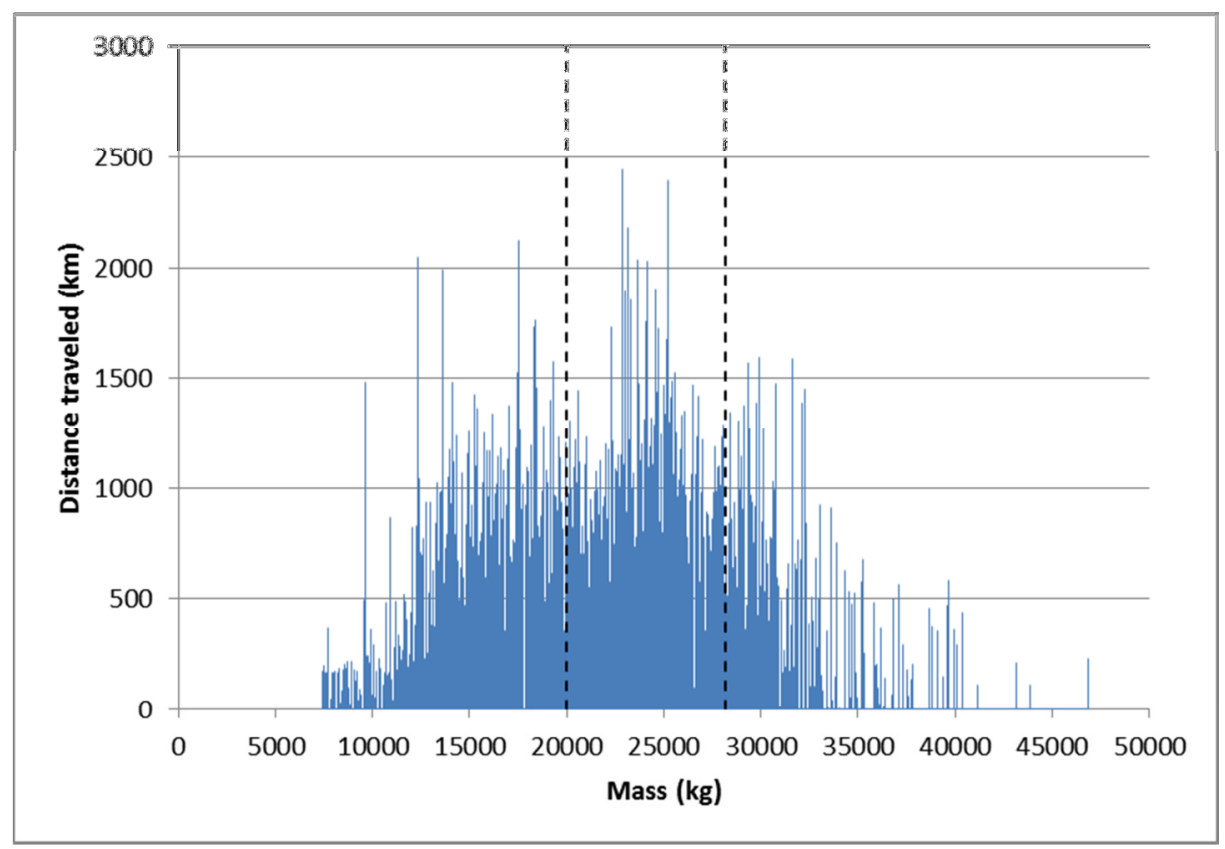

Figure 15 Distribution of masses for all segments of travel in the HTDC project.

The mass segments include cases where the trucks ran without the trailer attached (referred to as running "bobtail"), and this is represented by the lowest range of mass in the figure, around the empty tractor-only weight of about $8200 \mathrm{~kg}$ (based on specifications for the 2005 Volvo VNL tractor). The range of data shown in the figure around this mass level represents different volumes of fuel carried as well as the inaccuracy of the mass estimation method. The mass of an empty empty box trailer is about $7000 \mathrm{~kg}$, so the mass levels under about 15,000-16,000 kg (including fuel) represent the loaded cases. Again, there is some dispersion in the data due to the inaccuracy of the mass estimation used, so the delineation between empty and loaded cases is not as clear as the analysis suggests, but it does provide an estimate of the overall distribution.

The mass data determined above was used to analyze all of the drive cycle data for the project, and the mass distributions and tractive power values were calculated based on this data. The mass estimates were later questioned since the miles traveled with vehicle mass near the maximum gross vehicle weight limit of $80,000 \mathrm{lbs}$. $(36,300 \mathrm{~kg})$ were fairly limited, even though fleets generally attempt to operate with trucks as full as possible. Additional comparisons of measured vs. estimated mass were made to validate the mass levels. Unfortunately, it was found that the comparison done in the initial case discussed above was not representative of the overall mass level accuracy, and errors of up to 8500 $\mathrm{kg}$ were found in the mass estimates. This was discovered only after most of the other analyses were complete, and there was not enough time remaining in the project to re-evaluate the masses and rerun all of the analysis. Nonetheless, the project team wanted to understand the mass distribution as well as possible and estimate the probable error in the mass estimates. It was observed that the greatest difference between the estimated and measured mass occurred at the higher mass values, and the estimated mass almost always under-predicted the measured value. It is logical, therefore, to consider multiplying the estimated mass values by a constant value to see if a better match is obtained with the measured data. Six days of results for which the measured mass was available were considered, and 
there were 14 separate mass levels among these files. A constant multiple was applied to all of the data to adjust the original mass estimates, and the multiplier was optimized so that the error in the adjusted mass estimate is minimized on a root mean square (RMS) basis. The multiplier that minimizes the error was found to be 1.1265 , meaning that a roughly $13 \%$ increase in the estimated mass yields the best match to the measured data. The average absolute error after applying this correction is $2548 \mathrm{~kg}$. This value provides a measure of the uncertainty of the modified mass values. The data used to determine the mass multiplier are shown in Table 3, and the histogram of the masses using this modified value is shown in Fig. 16. With the mass adjustment factor applied to the data, we observe a cluster of cases in the histogram that are near the $36,000 \mathrm{~kg}$ level, i.e. close to the maximum gross load limit. This does not validate that the mass adjustment is correct, but the results do better agree with what one would expect than did the original estimate.

Table 3: Data used for the mass adjustment.

\begin{tabular}{c||ccc}
\hline $\begin{array}{c}\text { Truck \# } \\
\text { and date }\end{array}$ & $\begin{array}{c}\text { measured } \\
\text { mass }\end{array}$ & $\begin{array}{c}\text { estimated } \\
\text { mass }\end{array}$ & $\begin{array}{c}\text { adjusted mass } \\
\text { estimate }\end{array}$ \\
\hline \hline 1_20061102 & 29800 & 24577 & 27687 \\
& 14900 & 17503 & 19718 \\
& 30600 & 27729 & 31238 \\
\hline 1_20061103 & 29400 & 26688 & 30065 \\
& 15000 & 16625 & 18729 \\
\hline 2_20070207 & 32500 & 26750 & 30135 \\
& 16600 & 14600 & 16448 \\
\hline 3_20061222 & 21500 & 21650 & 24390 \\
& 14800 & 13942 & 15706 \\
& 30300 & 23336 & 26289 \\
\hline 3_20061224 & 30100 & 30241 & 34068 \\
\hline 3_20070103 & 21700 & 19372 & 21824 \\
& 15400 & 10369 & 11681 \\
& 31800 & 23278 & 26224 \\
\hline
\end{tabular}




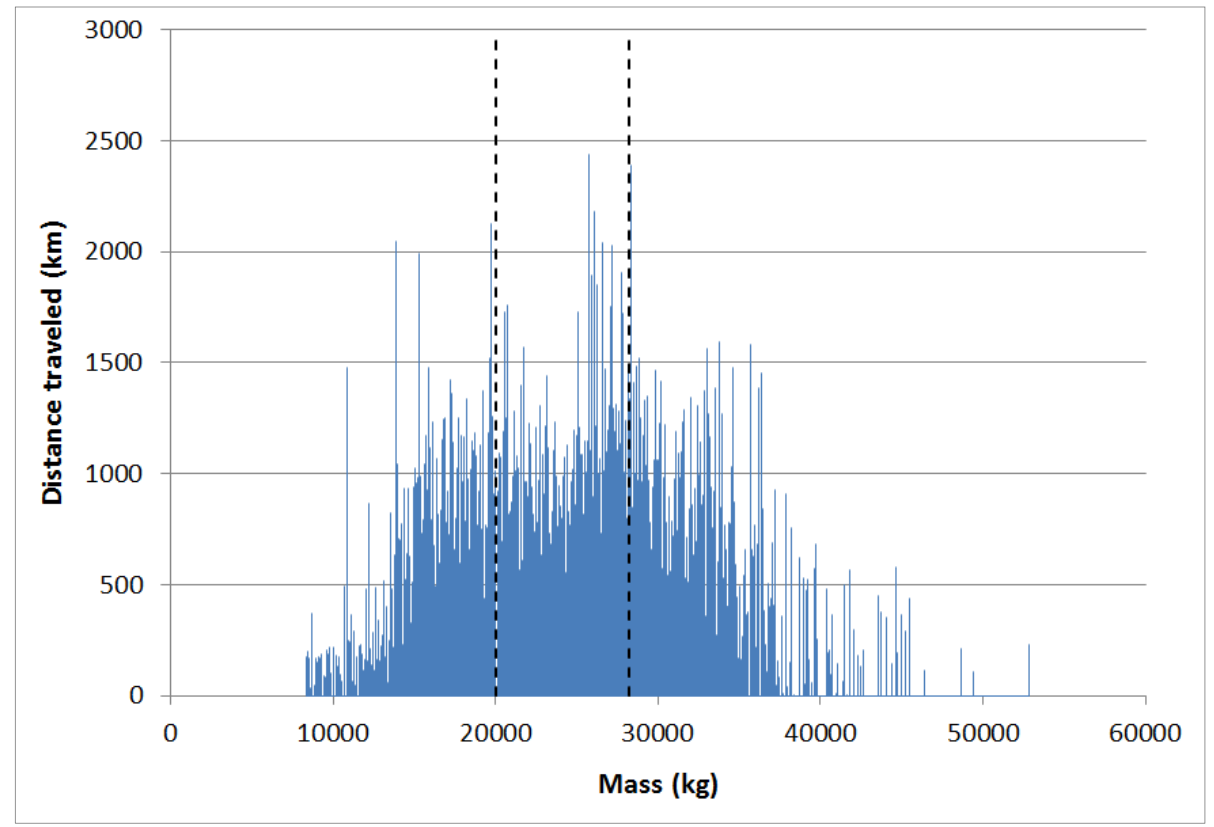

Figure 16 Adjusted mass distribution, based on a $12.65 \%$ increase compared to the original mass estimate.

It is evident that there were a fairly substantial number of miles driven either empty or with low loads being hauled. By considering the cumulative miles traveled as a function of load for the adjusted mass distribution, we found that about $6 \%$ of all miles traveled were driven with the estimated mass at or below $15,000 \mathrm{~kg}$, which is the approximate mass of the empty tractor-trailer. Before adjusting the mass estimates, roughly $13 \%$ of the cumulative mileage was at or below $15,000 \mathrm{~kg}$. The adjusted mass estimate yields an average overall mass of $24,774 \mathrm{~kg}(54,618 \mathrm{lbs}$.). Before applying the adjustment factor, the original distribution of masses gave an average value of 21,991 $\mathrm{kg}(48,482 \mathrm{lbs})$. This was calculated by weighting each estimated mass data point with the distance traveled along the segment at that mass to calculate a weighted mean.

\section{Synthetic Drive Cycle Creation}

The driving data measured in the HTDC project included 1,152,483 kilometers of travel while the six trucks ran for a total of 24,231 hours during a full year of measurements (these values represents the cleansed data, after removing corrupt data and incomplete drive segments, and is the total engine run time for the measurements, including idling). It would be extremely time consuming to analyze this quantity of data directly for any type of energy efficiency evaluation. As an alternative to completing a direct analysis of all the drive cycle data, a primary objective for this project was to reduce the driving data to create a set of synthetic drive cycles, of one hour or less in duration, that are representative of all of the data so that analysis can be performed using the more manageable length drive cycle(s). This section first explains what is meant by a "synthetic" drive cycle and the methodology used to develop one, and it describes the software ORNL has developed for this purpose. 


\subsection{The concept of a synthetic drive cycle}

As presented in section 2, the tractive energy is a primary factor in determining fuel consumption, and the tractive power required to move a vehicle forward at each instant in time is determined by the particular operating conditions experienced. As demonstrated by the equations developed in that section, the combination of speed, acceleration and grade, along with the parameters that characterize the vehicle configuration, uniquely determine the tractive power required at each instant in time. As a direct consequence of this correspondence between the vehicle operating condition and the tractive power, the fuel consumption is uniquely determined by the distribution of operating conditions that comprise the vehicle's usage history, i.e. its drive cycle. In reality, driving the same drive cycle multiple times with the same vehicle can result in somewhat different levels of fuel consumption since the driver may not shift at the same points in time, etc., so that even the same vehicle operating conditions can result in different engine operating conditions, which may result in small differences in the fuel consumption. This type of variation is secondary, however, to the tractive energy contributions, and we only address the tractive energy impact on fuel efficiency.

Since a vehicle's fuel efficiency is determined by the ratio of fuel consumed to the distance traveled, the vehicle usage can be scaled without changing the fuel efficiency. If we drive for two hours on flat ground at a steady speed of $80 \mathrm{~km} / \mathrm{hr}$. and then for two hours at $100 \mathrm{~km} / \mathrm{hr}$., we would consume twice as much fuel as if we drove only one hour at each speed (all other factors remaining equal). The fuel efficiency (or its reciprocal, the fuel economy) will be the same for either the one-hour or two-hour trip, however, since both the fuel consumption and the distance traveled will change by the same factor. If the same accelerations and decelerations occur during transitions from one speed to another in both cases, or if the fuel consumed in the speed transitions is negligible compared to the steady speed periods, it also would not matter if the higher speed operation occurs first or last. Similarly, the same tractive energy would be required if several segments of different lengths for the two speeds occurred in different orders during the trip (for example 15 minutes of driving at $100 \mathrm{~km} / \mathrm{h}$, followed by 20 minutes at $80 \mathrm{~km} / \mathrm{h}$, then 45 minutes at $100 \mathrm{~km} / \mathrm{h}$, and finally 40 minutes at $80 \mathrm{~km} / \mathrm{h}$ ), as long as the total ratio of time spent at each of the two speeds remained the same. Generalizing this idea, it can be shown, under the assumptions used in developing the tractive energy model, that two drive cycles will require the same tractive energy (for any given vehicle configuration) if the same set of operating conditions are experienced in both cycles and the fraction of time spent at each operating condition to the total cycle duration is the same in both cycles. This result is the basis for a characterization of drive cycles based on the distribution of all operating conditions experienced. This simple theorem also enables us to develop a drive cycle of a relatively short duration that will give the same fuel efficiency result as would be achieved from a much longer drive cycle (for example, during days or months of travel) simply by matching the distribution of operating conditions from the original drive cycle. This is the fundamental premise for the development of a drive cycle that accurately represents a given set of driving data, and we refer to the shortened cycle as a "synthetic drive cycle" since it represents a synthesis of all of the data contained in the larger set of driving data.

We note, in passing, that emissions from a vehicle are more complicated than fuel consumption since temperature plays a strong role in the chemistry governing engine emissions rates. When the engine 
and exhaust temperature are sufficiently high, however, the emissions, like fuel consumption, tend to be largely governed by the engine speed and torque. This allows "hot start" emissions to be determined in a manner similar to the fuel consumption, and a drive cycle that is representative for fuel efficiency purposes will also be representative with respect to hot start emissions.

\subsection{The Drive Cycle Generation (DCGen) Tool for developing a synthetic drive cycle}

ORNL has developed a set of tools for the analysis of measured drive cycles and the creation of synthetic drive cycles whose characteristics will be similar to an input set of driving data. These tools have been developed and refined during several projects including the Heavy Truck Duty Cycle (HTDC) project, the Medium Truck Duty Cycle (MTDC) project, and the current Truck Technology Efficiency Assessment (TTEA) project. The initial version of the Drive Cycle Generation (DCGen) tool used random sampling of microtrips (driving periods between two adjacent stops) from a single file of drive cycle data to generate a substitute drive cycle that was intended to be representative of the original. Various metrics such as average speed and acceleration, maximum speed, and number of stops per mile were used to characterize the relevance of the drive cycle generated. Convergence criteria based on these metrics could be set and the tool would repeat the process of its random selection of microtrips until the criteria were satisfied (when possible). It was found that this did not always provide drive cycles that led to consistent fuel economy estimates, even when the metrics of the reduced drive cycle were similar to the original cycle, and depending on the characteristics of the original drive cycle, the tool often did not find a converged result at the desired level of convergence. In subsequent versions of the software, different algorithms were developed to divide the input drive cycle into shorter segments and piece these together to better match the characteristics of the original drive cycle, and better means to characterize drive cycles based on the specific vehicle operating condition have been developed.

As discussed previously, matching the distribution of operating conditions from the original driving data is the primary goal in creating a synthetic cycle that will result in the same fuel efficiency, and the new drive cycle tools focus heavily on matching the distribution of vehicle operating conditions. For the tractive energy model, the operating condition at each point of time is comprised of the speed, acceleration, and the grade. It is challenging to visualize and identically match a distribution of three variables, but by considering the bivariate distribution of the speed and acceleration, and using real grade conditions occurring simultaneously to the speed and acceleration points, the overall distribution should be very well approximated. This is the approach that has been followed for the synthetic drive cycle creation. An example of a speed vs. acceleration bivariate histogram from a single day of driving is presented in Fig. 17. The bivariate histogram shows the cumulative duration of time that the vehicle was driven at each operating condition. Each bin represents a range of speeds and accelerations, and is defined by discretizing the full range of the speeds and accelerations from the drive cycle. The color of each bin corresponds to the number of times during the drive cycle that the speed and acceleration were within the range represented by that bin. The scale at the right of the figure indicates the total time duration experienced at each operating condition in the drive cycle. By representing a drive cycle in this manner using the speed-acceleration distribution, the time order of the operating conditions is eliminated. The distribution can also be scaled so that the overall duration is not a factor. 


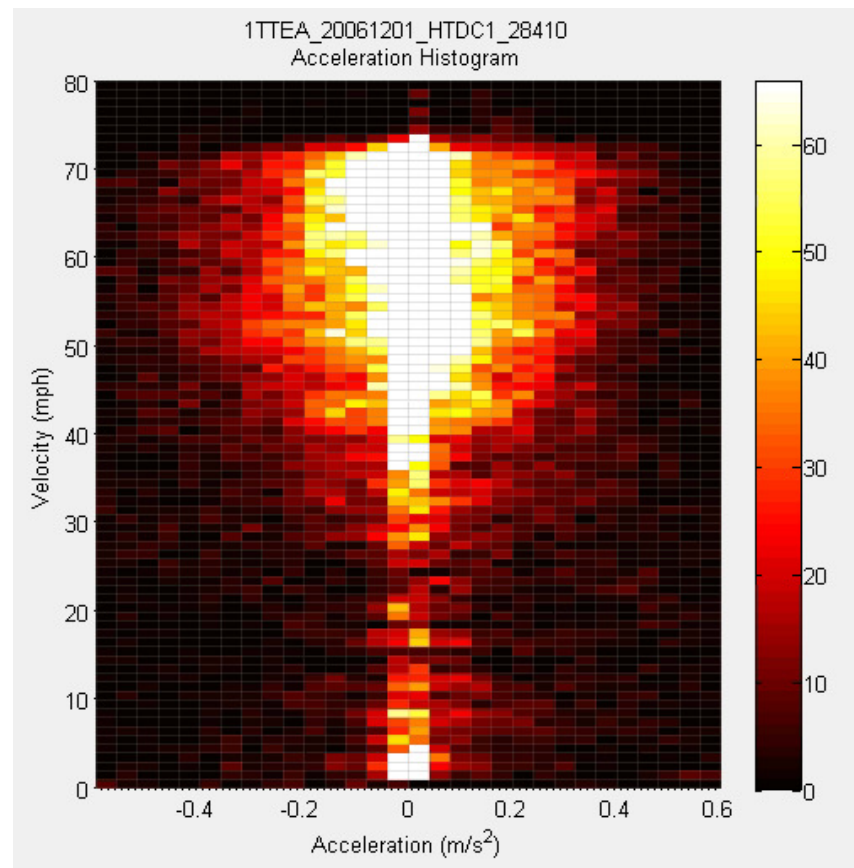

Figure 17 Bivariate histogram of the velocity and acceleration for a day of driving.

The drive cycle analysis for this project required a high degree of accuracy for matching the accelerationspeed distribution of the synthetic drive cycle to that of the complete measured data sets. A new version of the DCGen tool was developed in which the user manually selects drive cycle segments using a target speed-acceleration distribution generated from the complete set of driving data. This version of the tool consists of three separate Matlab-based programs, which use many of the same functions incorporated in earlier versions of the automated DCGen tool, but relies on the user's judgment to create the synthetic cycle as opposed to having it generated using a purely algorithmic approach. The following describes the function and use of each program module in the DCGen tool.

The first program module provides an interface that allows the user to load any number of drive cycle files, and the distributions from each file are combined into a "total" bivariate histogram that represents the concatenation of all of the input files. The individual drive cycle files can be reviewed and processed for further use in the subsequent modules of the software, as needed. Reduction of the data that is stored from each file is necessary when using large data sets, and the user must select the individual histograms or files he will use in the other modules of the DCGen tool. For subsequent analysis in creating the synthetic drive cycle, it is necessary to break the file into discrete drive cycle segments, and the user must launch the segmentation function for any files that will be used as basis functions in the synthetic cycle creation. Typically, histograms that are similar to that of the entire data set are selected in order to make the matching of the histograms more efficient, but the user has full control of the selection process. The software allows the user to segment additional files at any time in the synthetic cycle creation if it is found that portions of the total histogram are not represented by segments that were initially selected. The user interface for the first software module of the DCGen tool is shown in Fig. 18. 


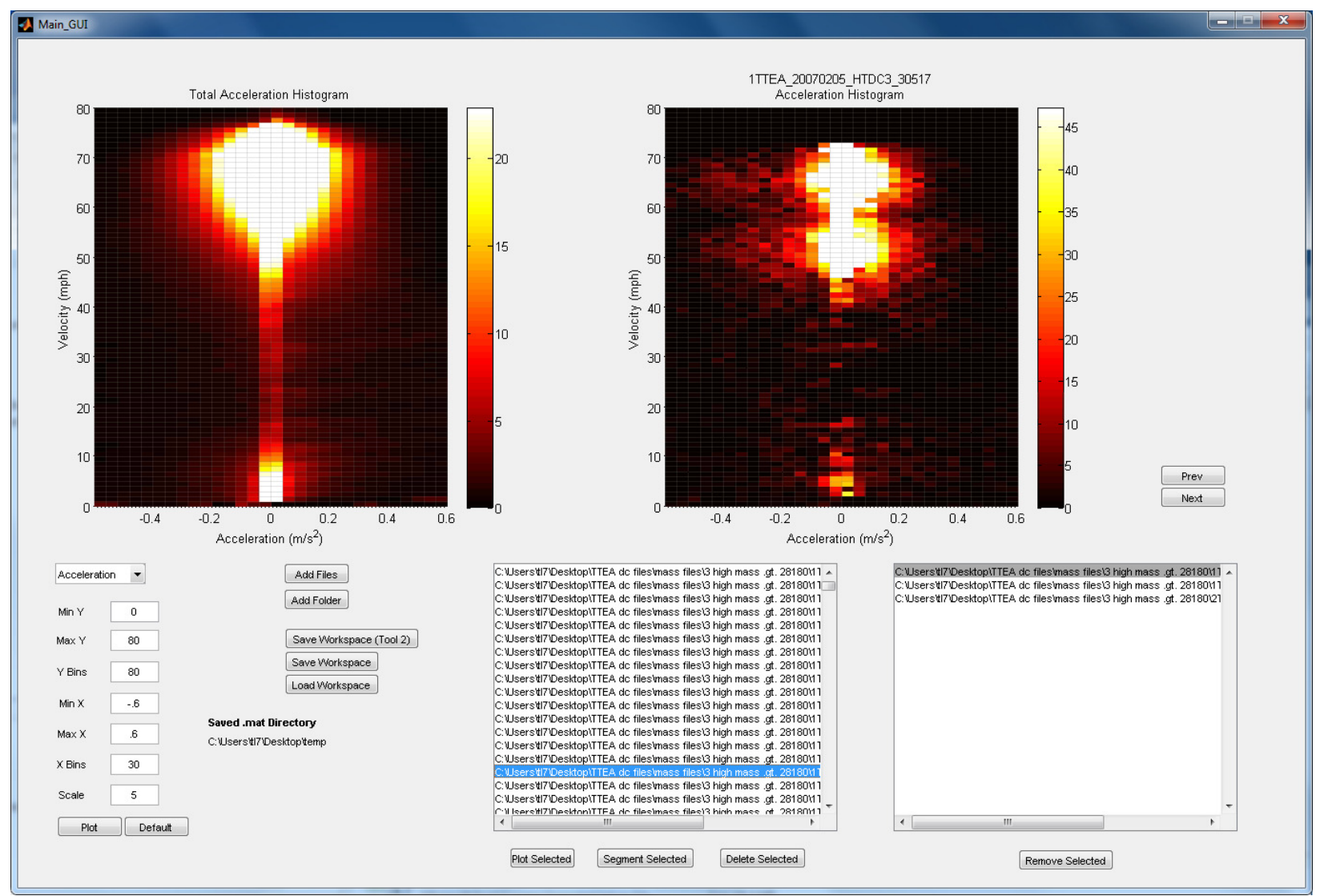

Figure 18 The DCGen tool module 1 interface.

Once the user has selected the desired set of files and created the total histogram representing all of the driving data to be analyzed, the data is processed to create individual drive cycle segments that will be used in subsequent steps of the synthetic cycle creation. These segments are loaded into the second program module, which allows for specific segments to be selected from each file for inclusion in the synthetic cycle. The user must select segments one at a time in order to generate a tentative synthetic histogram (which is simply an accumulation of the individual segments' histograms) that matches that of the complete data set. The tool manages the accounting of the synthetic histogram as segments are selected and presents a histogram representing the difference between the total histogram, scaled to a user-selected target cycle length, and the segments selected for inclusion in the synthetic drive cycle. The user's objective when creating the synthetic drive cycle, therefore, is to achieve a difference histogram that approaches zero. The difference histogram and data for an individual segment, as they are presented in the second software module during the selection process, are shown in Fig. 19, and the corresponding segment speed and elevation are shown in Fig. 20. The software has been developed to automate the search for segments meeting specific criteria of speed and accelerations, and the portions of the histogram for which additional data points are required can be quickly identified and matched with the available segments. The interface for the search function is shown in Fig. 21 . Functions to automate the process of identifying appropriate drive cycle segments for inclusion in the synthetic cycle were implemented in the software to improve the efficiency of the synthetic cycle creation. 

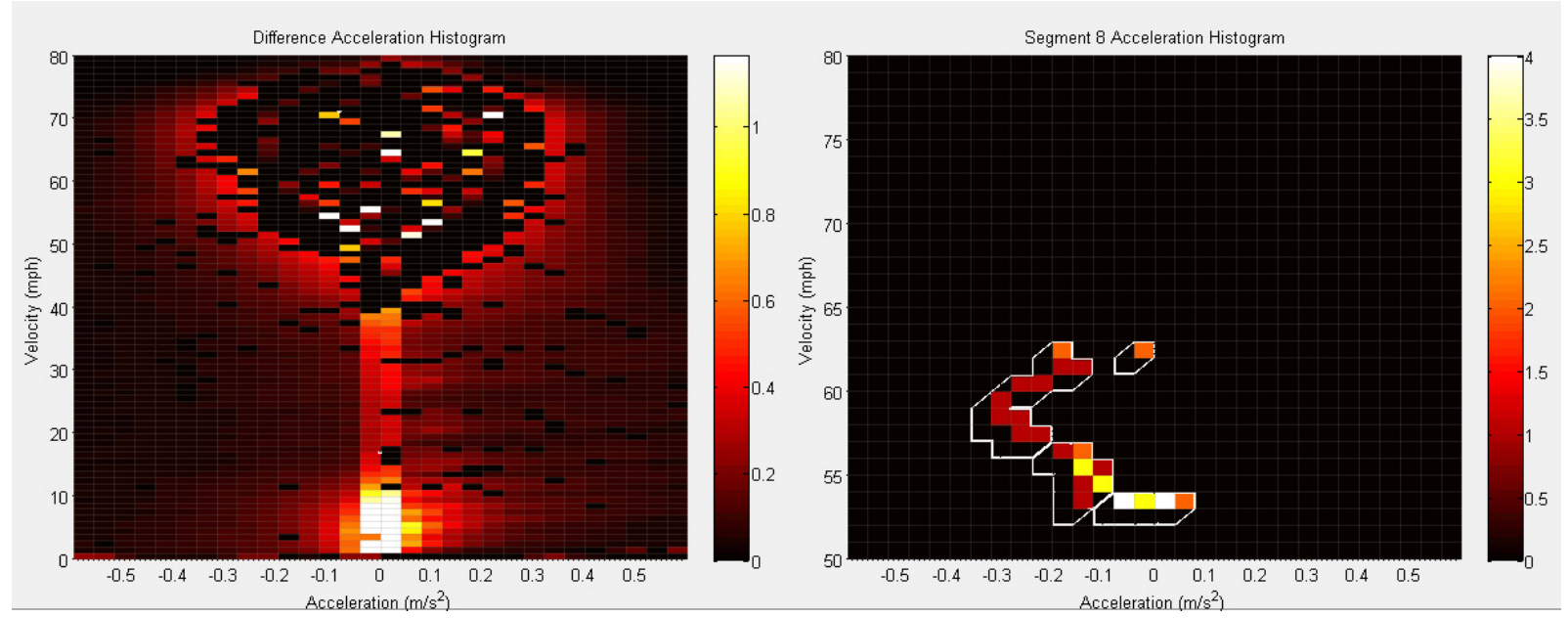

Figure 19 Difference and segment histograms during the creation of a synthetic drive cycle.

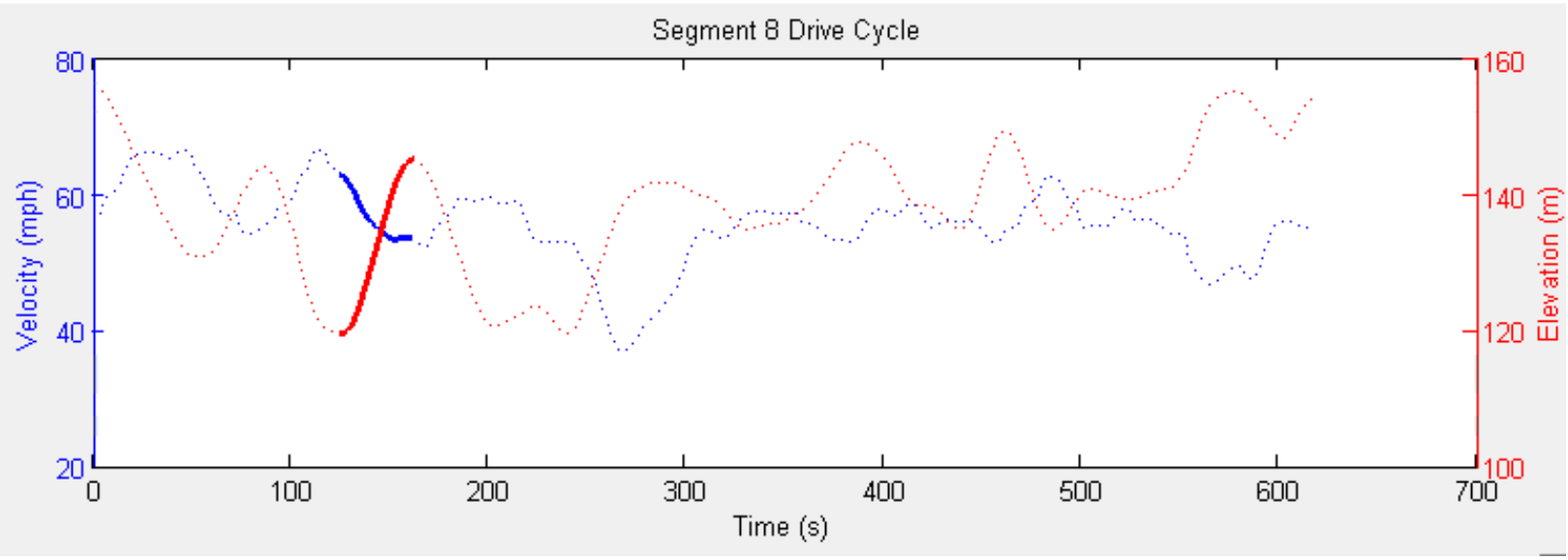

Figure 20 Plot of a single drive segment in DCGen tool program module two, showing the speed and elevation changes.

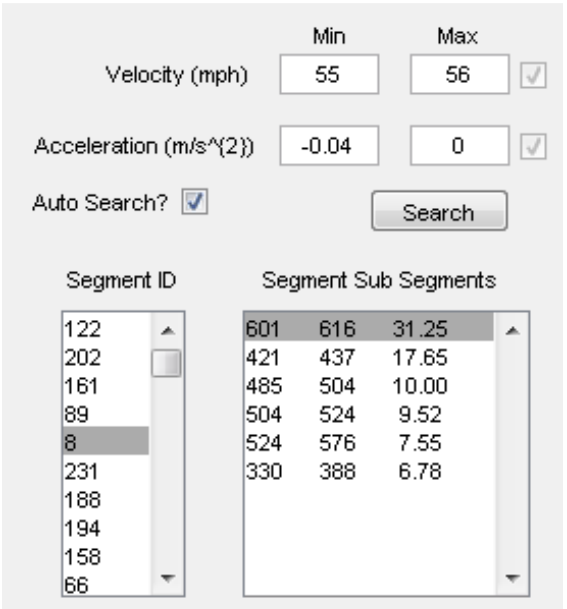

Figure 21 DCGen tool, second module segment search interface. 
After selecting drive cycle segments so that the tentative synthetic histogram is as similar as possible to the original histogram, the third program module is run for the final creation of the synthetic drive cycle. The main goal of this module is to arrange the drive segments selected in the second program module to create the final synthetic duty cycle and to ensure that the speed and elevation profiles comprising the synthetic cycle are continuous and smooth. This is done by matching the starting and ending speeds between subsequent segments and inserting or removing points as needed. Final refinements of the synthetic cycle histogram are also made using this module so that the synthetic histogram matches the total histogram as closely as possible. The program incorporates the elevation data corresponding to each selected segment (if it was present in the original data files) and integrates this data into the final synthetic drive cycle. A completed synthetic drive cycle is shown in Fig. 22. The individual segments included in the cycle can be seen in alternating colors in the velocity and elevation profiles.

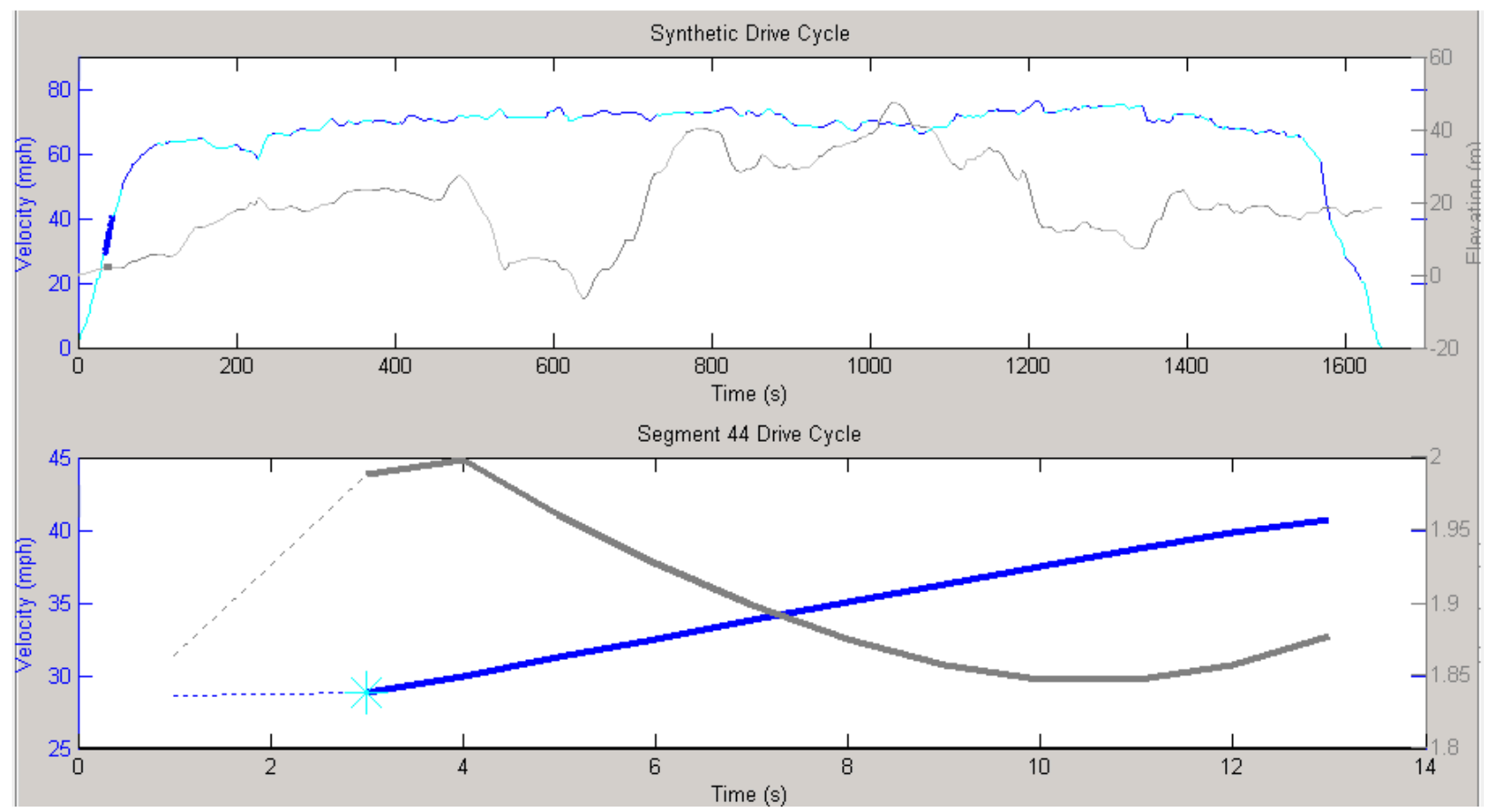

Figure 22 Manually-generated DCGenT synthetic plot.

When the segments from module two are initially arranged, the speed data inevitably contains some gaps between segments so that the speed profile is not continuous. To remedy this problem, the user can rearrange segments for a better match of endpoints, and he may add or modify points at the beginning or end of any segment in order to create the desired continuous cycle. The interface used to edit data for a segment is shown in Fig. 23. 


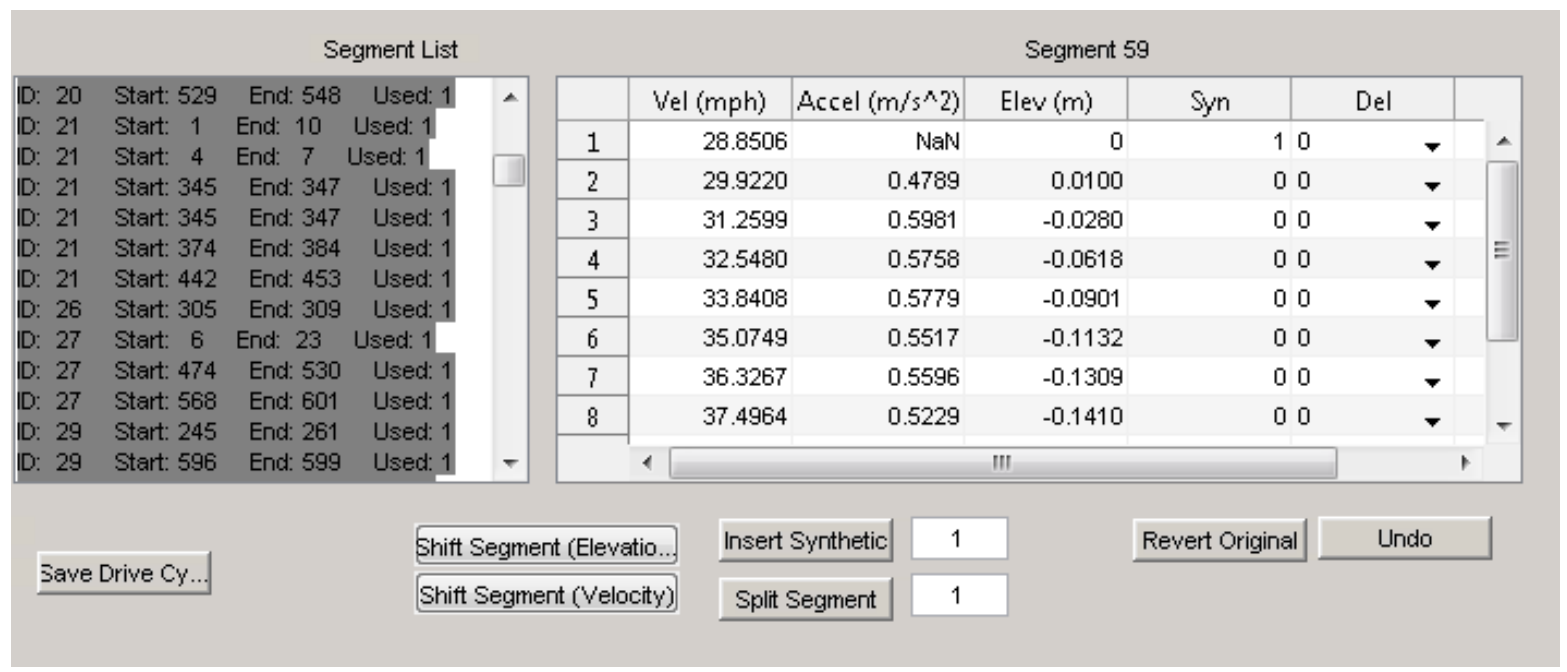

Figure 23 Manual DCGenT segment data.

Once all of the segments have been combined for the synthetic cycle and gaps between segments are corrected, the synthetic histogram is created and compared to the original data set using the total histogram. The user must make any final adjustments to the synthetic drive cycle to correct errors in the synthetic histogram that develop during the creation process. Figure 24 shows a comparison of the original (total) histogram and the synthetic histogram for a completed synthetic drive cycle. (The speed range presented is truncated for clarity.)
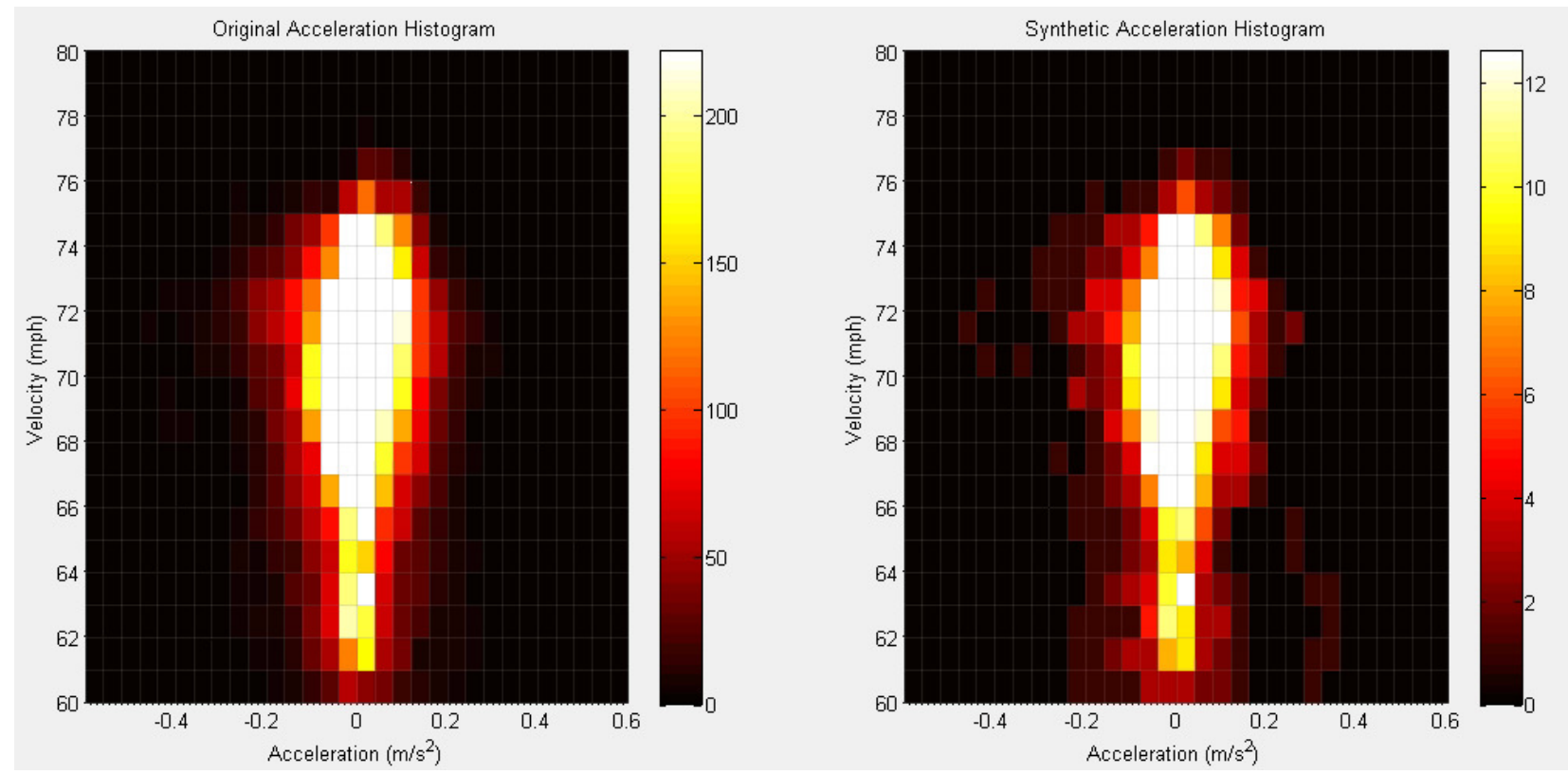

Figure 24 Manual DCGenT synthetic histogram.

This implementation of the DCGen tool was developed to create synthetic drive cycles that very closely represent any set of driving data. The synthetic cycle can be generated to have a length that is appropriate for use in tools such as PSAT and Autonomie for modeling vehicle systems, or for testing purposes. While an original data set of even a full day's worth of data would require an excessive 
amount of time to process with some vehicle models, the DCGenT can be used to create synthetic duty cycles and these cycles can be run significantly faster and will yield the same fuel economy result. Since building hardware can be expensive, the modeling approach using real data is beneficial to not only researchers for testing aerodynamic and other fuel saving technologies without physically instrumenting a vehicle, but also to truck manufacturers and truck fleets for selecting vehicles tuned to their fleet's specific duty cycle.

\subsection{Validating the Use of a Synthetic Drive Cycle and the Tractive Energy Model for Predicting the Fuel Efficiency Benefits from Advanced Vehicle Technologies}

The synthetic drive cycle development methodology and software have been described and a basic theory was presented to explain why a synthetic cycle used with the tractive energy method will yield the same results as would be attained using the complete set of drive cycle data. In this section, the ability of the synthetic drive cycle to represent a larger data set is validated by comparing results from approximately 8 hours of driving to those from a synthetic drive cycle developed to represent the full data. A comparison of the energy savings estimates was made with the tractive energy model as well as by comparing the predicted fuel economy using Autonomie vehicle performance software.

Figure 25 shows measured driving data from a complete day of driving during which 858.9 km were traveled over 28,787 seconds of vehicle operation. This data was used to generate a synthetic drive cycle with the DCGen tool, as described in the previous section. The original drive cycle was reduced to a synthetic cycle with a duration of 1645 -seconds. The synthetic cycle representing the original cycle is shown in Figure 26.

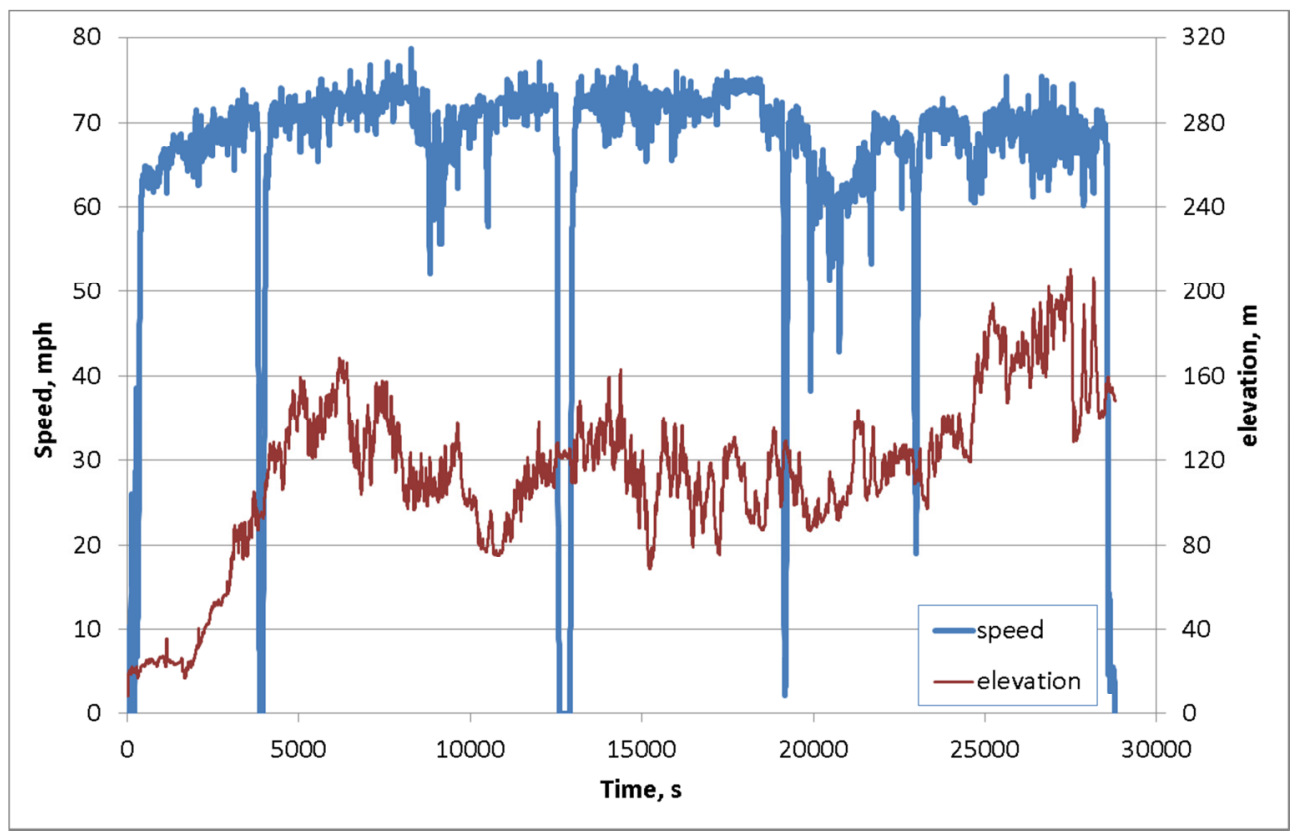

Figure 25 Driving data used to develop the validation synthetic drive cycle. 


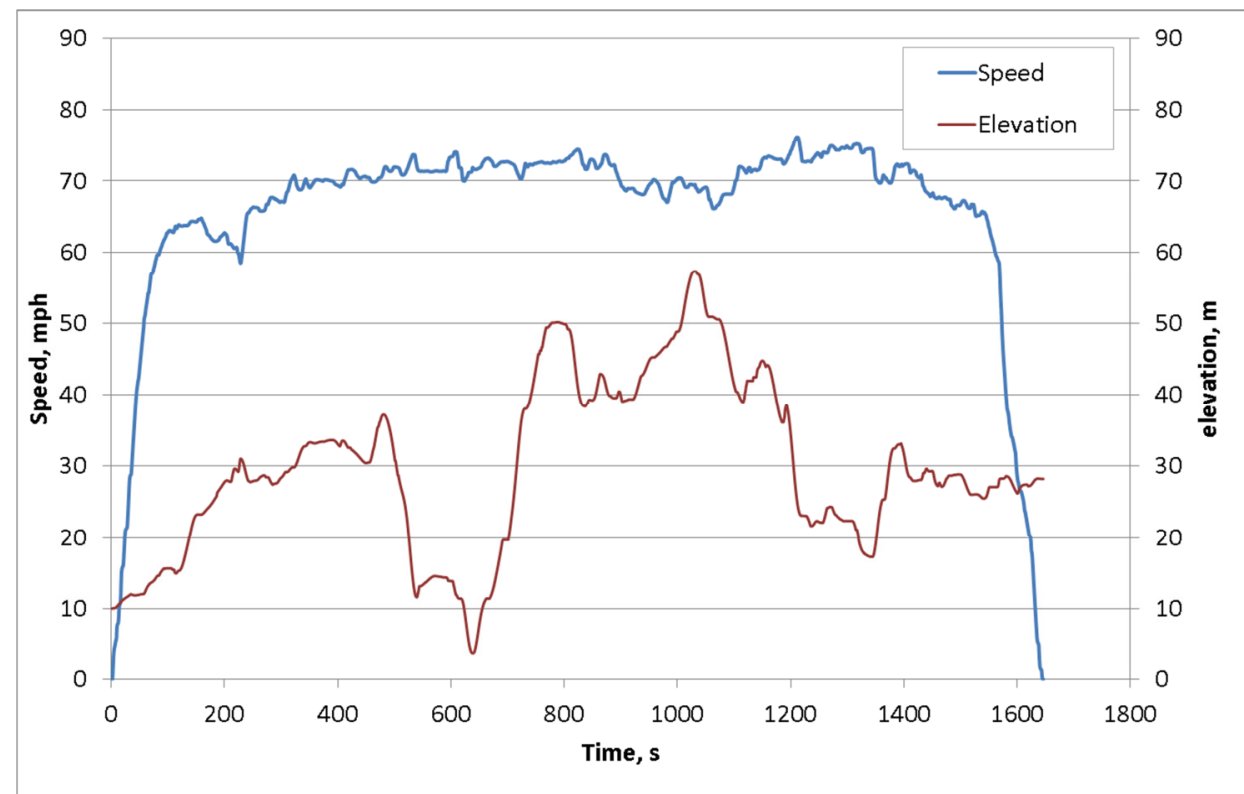

Figure 26 Synthetic drive cycle developed from the driving data presented in Fig. 25.

Figure 27 compares the bivariate velocity vs. acceleration distributions for the original and synthetic drive cycles. Due to the short duration of the accelerations to and decelerations from highway speeds relative to the length of the rest of the drive cycle, the number of occurrences for each bin in the histograms corresponding to the low-speed accelerations/decelerations is very low. The bins do not even appear in the histograms when using a normal range of scales, and the low speed range was omitted in Fig. 27. This low incidence of the low speed bins indicates that the corresponding operating conditions are rather insignificant in characterizing the drive cycle.
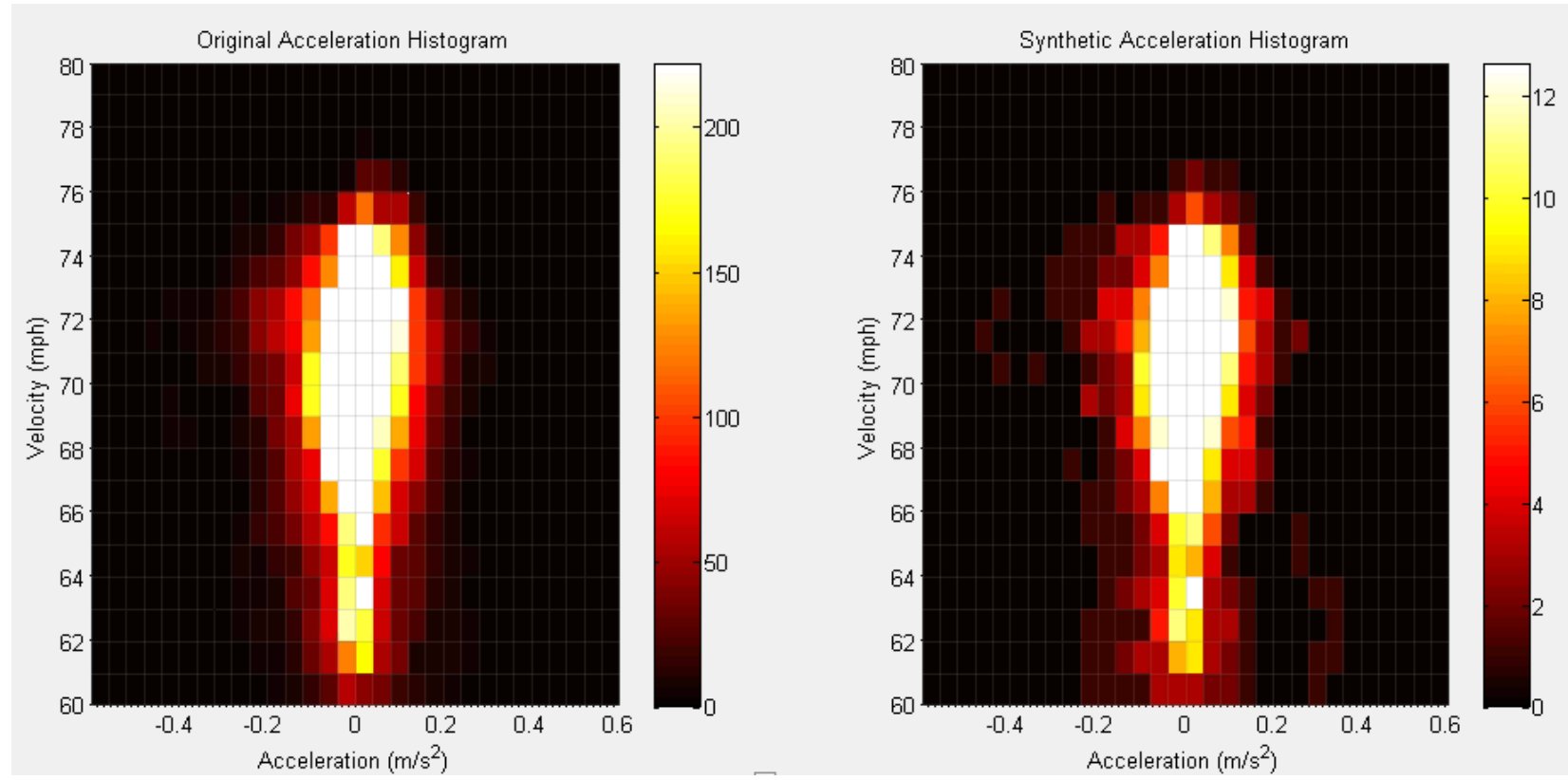

Figure 27 Bivariate histogram of the original driving data and of the synthetic drive cycle developed for the validation. 
Figure 27 shows that the histogram for the validation synthetic drive cycle matches the original cycle extremely well for the dominant operating conditions experienced. There are a few bins that differ slightly in magnitude from the original histogram. Since scaling the original cycle's bivariate histogram for the reduced length of the synthetic drive cycle results in bins in the target bivariate histogram that contain non-integer time durations, while the synthetic drive cycle was developed using one-second time intervals, it is not possible to have an identical match between the two cycles. Furthermore, even in cases where a better match may be numerically possible, the process of creating the synthetic cycle using the DCGen tool does not always lead to an ideal solution. In spite of the differences, the overall agreement between the original and synthetic histograms is considered to be excellent.

To compare the low-speed operating conditions for this cycle, the scale was adjusted so that most of the bins are visible in the histogram plots, as shown in Fig. 28. It is evident that many conditions in the synthetic cycle with low speeds show a higher occurrence than the corresponding points in the original drive cycle. This is again a result of the fact that only integer time durations are included in the drive cycles, and bins with low frequencies of occurrence cannot be reproduced very accurately by the scaling process used in the synthetic drive cycle creation. Since the lower speed region must be traversed by accelerating and decelerating at finite rates, some points in the synthetic cycle must be present in these ranges. It can be seen that a fairly broad range of accelerations were covered at each speed in the original drive cycle, although each operating condition only has a low frequency of occurrence. The distribution of accelerations can be represented in a coarse manner in the synthetic cycle by ensuring that a similar range of accelerations occurs in the synthetic cycle, at least on average across several speed intervals. In creating the synthetic cycle, an attempt was made to do this by including accelerations that occurred in clusters in the original cycle into the final synthetic cycle. For example, if there are 5-10 points over a $10 \mathrm{mph}$ range of speeds for which the individual occurrence in the original cycle is $0.1-0.25$, then including a single point in the synthetic drive cycle at one speed within this range provides a means to represent the original accelerations in a general sense, within the limitations of the 1-second intervals used for the synthetic drive cycle. In any event, the low speed operating conditions for this cycle represent only a small portion of the overall distribution, and the effect on the fuel consumption of these variations relative to the original drive cycle are relatively small. We note that the same approach of including data to represent, in an average sense, operating conditions that occurred at low levels of occurrence over multiple speed levels or across several acceleration bins was used not only for the low speed range, but also for the highway operations at the higher acceleration level. This is why some bins for the synthetic cycle histogram in Fig. 27 are populated even though the corresponding bins in the original histogram appear to have no occurrences for the same operating condition.

With the synthetic cycle completed, a validation to demonstrate the accuracy of fuel economy results based on the synthetic cycle development was completed by performing simulations of fuel economy using Autonomie vehicle performance software with the two drive cycles shown above, original and synthetic. Autonomie is a plug-and-play vehicle model architecture designed to simulate vehicle performance and evaluate powertrain technologies for improving fuel economy [7]. It estimates fuel consumption by applying a detailed physical representation of all of the major powertrain components 
on the vehicle, and links the forces required to propel the vehicle with an engine map, which characterizes the fuel consumption of the engine as a function of engine speed and torque. An Autonomie vehicle model has been developed at ORNL to represent the vehicles used in the HTDC study. A heavy duty diesel engine map available in Autonomie was used in the model, but the engine parameters were tuned to be representative of the 15-liter, 6-cylinder Cummins ISX 475 diesel engine that was present on the HTDC test vehicles. The transmission in the model is a 10-speed manual transmission, which is also representative of the tractors with a manual transmission used in the study. The mass simulated in the Autonomie evaluation was $22,000 \mathrm{~kg}$, which was the mass estimated for the measured driving segment. Other parameters in the model were selected to be consistent with those used in the tractive energy analysis. Specifically, the truck's aerodynamic drag coefficient was taken to be $\mathbf{0 . 6 2}$, while a constant value of 0.007 was used for the coefficient of rolling resistance in the analysis. The same vehicle model configuration has been used in prior evaluations to represent the same HTDC test vehicles, and this model has been validated against measured fuel consumption results at ORNL.
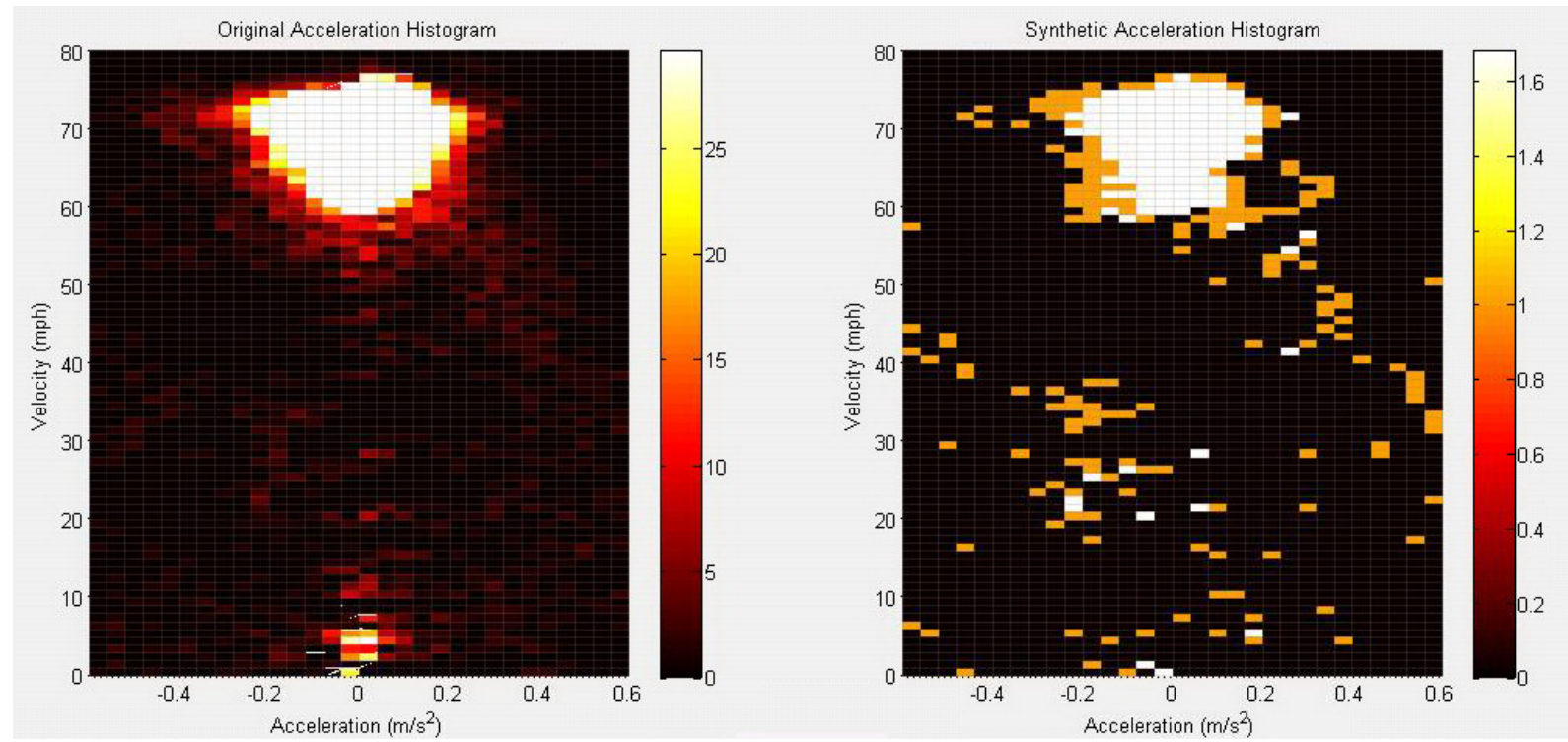

Figure 28 Original and synthetic histograms shown with the scale modified to highlight the low speed operating conditions.

The model was run for both the original drive cycle and the synthetic cycle, which is intended to closely represent the original cycle. The fuel economy values predicted using the two drive cycles were 5.62 and $5.57 \mathrm{mpg}$, respectively. This level of consistency in the predicted fuel consumption (within 1\%) gives a high level of confidence that the synthetic drive cycle is highly representative of the original cycle and that the fuel economy estimate using a carefully constructed synthetic drive cycle can yield results that are very close to those from the full set of driving data that the synthetic cycle represents.

In addition to performing the fuel economy analysis with Autonomie, the tractive energy model was run using both drive cycles to determine the relative contributions from each energy loss factor to the total tractive energy, in addition to evaluating the fuel savings expected with various combinations of technologies based on the tractive energy method. The contributions of each energy loss factor to the driving tractive energy, given by Eqs. (4), (5) and (7), are expressed as a percentage of the driving 
tractive energy (see Eq. (8)). The relative contribution from each energy loss term is strongly dependent on the drive cycle, so this comparison is an excellent test of how well the synthetic cycle matches the original driving data. These terms are also the basis for the energy saving estimates using the tractive energy model, so their accuracy is critical to that of the tractive energy predictions. Table 4 shows the results obtained for the energy loss factors based on the tractive energy model.

Table 4: Comparison of results for the energy loss factors in the tractive energy model, for the original and synthetic drive cycles from the validation case.

\begin{tabular}{|l|l|l|}
\hline $\begin{array}{l}\text { Energy Loss Factors (Expressed as a } \\
\text { Percentage of the Total Driving Tractive } \\
\text { Energy }\end{array}$ & $\begin{array}{l}\text { Original Drive } \\
\text { Cycle }\end{array}$ & $\begin{array}{l}\text { Synthetic } \\
\text { Drive Cycle }\end{array}$ \\
\hline $\begin{array}{l}\text { Brakes (corresponding to regenerative braking } \\
\text { potential) }\end{array}$ & $3.0 \%$ & $4.0 \%$ \\
Aerodynamic drag, driving & $55.7 \%$ & $54.8 \%$ \\
Aerodynamic drag, braking & $3.3 \%$ & $2.5 \%$ \\
Rolling Resistance, driving & $35.1 \%$ & $34.8 \%$ \\
Rolling Resistance, braking & $2.1 \%$ & $1.9 \%$ \\
\hline
\end{tabular}

It is found that all of the factors calculated using the synthetic drive cycle are within $1 \%$ of those from the original drive cycle, which indicates that the prediction of the fuel saving potential of each advanced efficiency technology (and their combinations) when using the synthetic drive cycle will be very consistent with the result based on the original driving data. Since the elevation changes were not forced to be proportional when creating the synthetic drive cycle, there is a slightly greater increase in the potential energy in the synthetic drive cycle than occurred in the original drive cycle. The fact that the potential energy is non-zero in both cases (it represents $0.8 \%$ of the driving tractive energy for the original drive cycle and $2.0 \%$ for the synthetic cycle) is the reason that the relative energy contributions of the other terms do not sum to $100 \%$. The impact of this small difference in the potential energy change is minimal on the overall results, although it is responsible for some of the differences occurring in Table 4.

The tractive energy analysis was run for both cycles to quantify the fuel savings potential associated with reductions in mass, rolling resistance and/or aerodynamic drag. The fuel savings estimates are presented in Fig. 29. Note that according to the assumptions of the tractive energy model, the estimated energy savings from combinations of the technologies are additive when considering either of the two cases independently, i.e. with regenerative braking or without regenerative braking. Due to the effect of the braking tractive energy contributions, however, the energy savings associated with each energy loss factor are different depending on whether regenerative braking is used or not. The only noticeable difference in the results between the predictions made with the tractive energy model for the two drive cycles is the energy savings associated with regenerative braking for this case. 


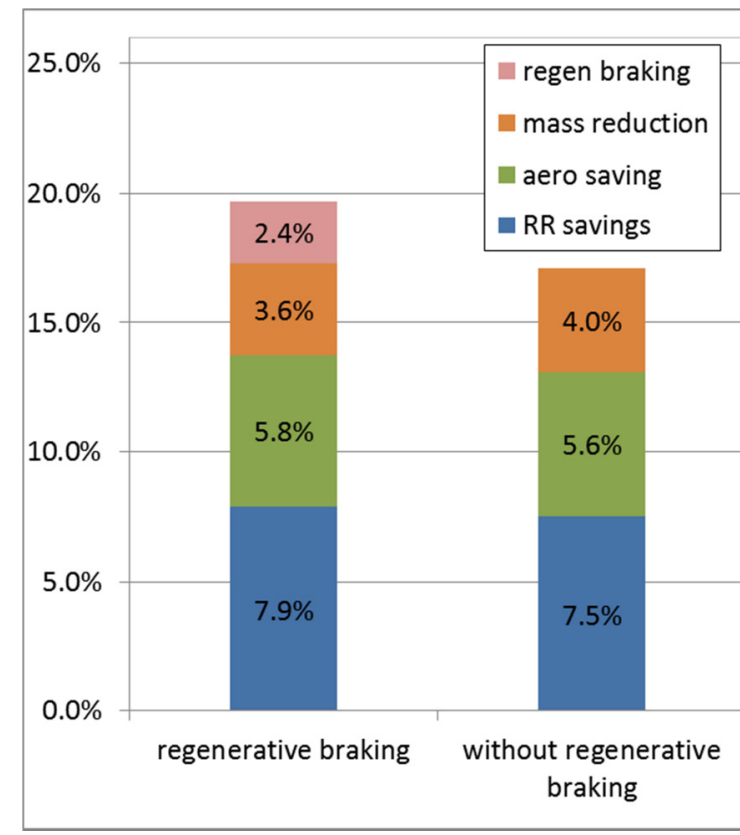

(a) Original Drive Cycle

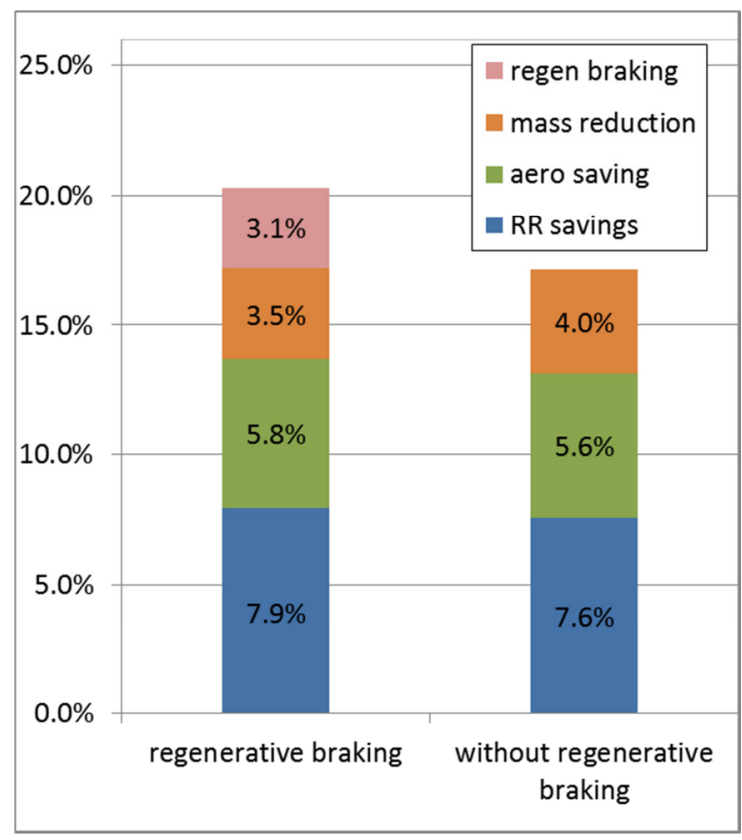

(b) Synthetic Drive Cycle

Figure 29 Relative Fuel Savings estimates based on the tractive energy model for (a) the original drive cycle and (b) the synthetic drive cycle. The variations evaluated were for a mass reduction of $2000 \mathrm{~kg}$, a reduction in $C_{R R}$ by 0.0015 and a $10 \%$ reduction in $C_{d}$. The efficiency of the regenerative braking system is assumed to be $80 \%$.

Table 5 shows the default values used in the tractive energy analysis for all of the primary parameters in the model. The same assumptions are used in all of the tractive energy evaluations presented in this report. These values were selected to be representative of typical class 8 tractor-trailers. The variations considered were selected to correspond to the level of reduction that could be achieved when advanced efficiency technologies are employed on an initially non-optimized vehicle configuration. A mass reduction (corresponding to vehicle lightweighting) of $2000 \mathrm{~kg}$ is assumed in the reduced mass scenario. The energy savings result is based only on the fuel efficiency improvement resulting from the decreased mass, and it is assumed that the payload does not increase as a result of the mass reduction. The rolling resistance coefficient reduction employed in the analysis is 0.0015 (also referred to as $1.5 \mathrm{~kg} / \mathrm{ton}$ ), which is a typical level of reduction that can be realized when converting from conventional dual tires to New Generation Wide Base Single (NGWBS) tires. The reduction in the coefficient of aerodynamic drag assumed in the analysis is $10 \%$, which is a level of reduction that could be achieved in tractor-trailers if implementing aerodynamic drag reduction devices such as trailer skirts, etc. The same levels of reduction will also be used in all of the tractive energy analysis cases.

Table 5: Default values for parameters used in the tractive energy analysis.

\begin{tabular}{|l|c|c|c|c|c|c|c|}
\hline & $C_{d}$ & $C_{R R}$ & $\eta_{\text {eng }}$ & $\eta_{\text {trans }}$ & LHV, MJ/L & $P_{\text {access }}, k W$ & $\eta_{\text {regen }}$ \\
\hline Default values & 0.62 & 0.007 & 0.42 & 0.9 & 35.8 & 14.9 & 0.80 \\
\hline
\end{tabular}




\section{Tractive Energy Analysis Results Based on the Synthetic Drive Cycle}

\subsection{The synthetic drive cycle corresponding to the overall fleet usage}

For the analysis of the complete driving data from the HTDC project, the data files were processed as described in Section 3 of this report. We began to process the data corresponding to the low, medium and high mass levels separately using the DCGen tool, but the results from the first module analysis showed that there was very little difference in the speeds and accelerations experienced for the three mass levels. It was expected that there would be a non-negligible decrease in accelerations with increasing mass since the power-to-weight ratio decreases with greater load. The data indicated very little difference between the three cases, however, so the drive cycle developed for the medium mass case was used to analyze all three mass levels. The tractive energy analysis was still repeated for each case separately, however, to account for impact that the difference in mass has on the fuel efficiency evaluations. Figure 30 shows the comparison of the distributions of speed vs. velocity, based on the bivariate histogram, for the three mass levels considered. Careful comparison of the three cases does reveal some differences between them, but the overall profile is surprisingly similar. It is hypothesized that highway conditions, for which most of the range of accelerations are experienced, cause the accelerations to be limited by aerodynamic drag to a point that the mass differences play a relatively minor role. Accelerations also tend to be limited by traffic conditions, so that in many conditions it may not be possible to accelerate at the maximum level that the engine is capable of. Another possible mitigating factor is driver training. Since drivers are trained to only accelerate gradually so that the best efficiency can be achieved, this will tend to reduce the higher accelerations that could be achieved when a lower vehicle loading is present. The fact that the class 8 tractor-trailer application operates primarily on the freeway with quite limited low speed operations very likely influenced this effect significantly, since accelerations at lower speeds probably do show greater acceleration variations with load.

It is interesting to observe that the dominant accelerations for the low speed operations are still at very low levels. This effect was examined in some detail to understand the cause and verify that there was not a problem with the software. Since the distribution shown is scaled to only show the operating conditions that are most frequent in the drive cycle, many of the operating points at higher accelerations, while still present, are overwhelmed by the lower acceleration conditions. For a segment of driving such as that analyzed for the validation synthetic cycle from the previous section of the report, the low speed-high acceleration operating conditions are still apparent, even though their contribution to the overall drive cycle is rather limited. When all driving data is included together, however, the periods of driving at relatively steady, low to medium speeds end up being much more significant than the higher acceleration conditions at the same speed. If a truck drives for just 30 minutes at a speed range of 30-40 mph and maintains a fairly stable speed, this will generate over 1500 seconds of low acceleration conditions for this speed range. This compares to only a few seconds at a time of data within any given speed range that is generated when the truck accelerates from a stop to highway speeds or decelerates rapidly after exiting the highway. There are enough operating conditions on secondary roadways in this application when the trucks drive at steady speeds that this is much more dominant than the high acceleration operations that take place. In the full distribution, as contained in the speed-acceleration histograms, there are thousands of data points for accelerations up to and 
beyond the $0.2 \mathrm{~m} / \mathrm{s}^{2}$ level, but these thousands of points do not register significantly in comparison to the millions of data points contained in the overall distribution. One can see an "aura" of low density operating points surrounding the main portions of the drive cycle, but these account for a rather small percentage of the total vehicle usage. Using the statistics of the very large data sets from the HTDC project, the analysis identifies the portions of the drive cycle that are most representative of the overall usage, and it is precisely this information that this drive cycle analysis and synthetic cycle development aims to capture.

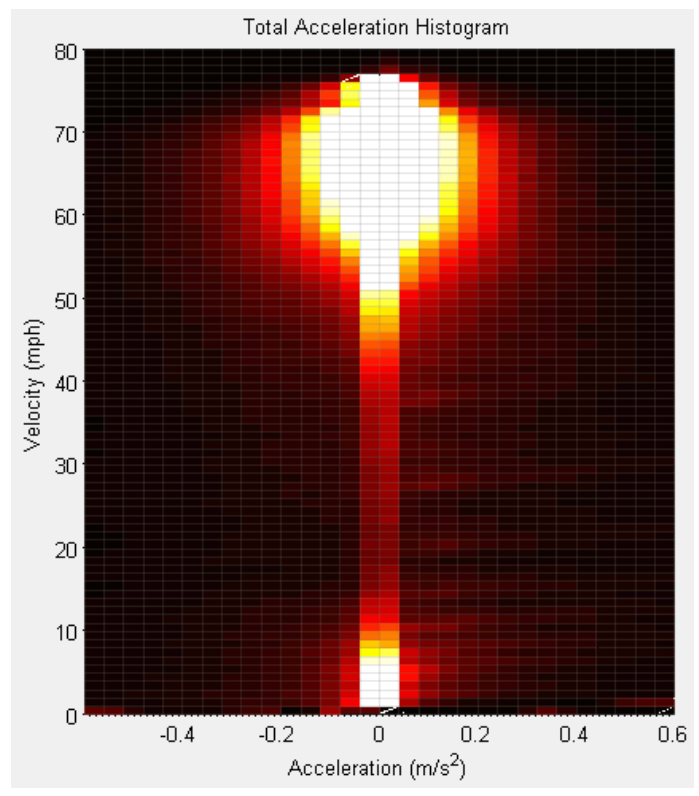

(a) Low mass

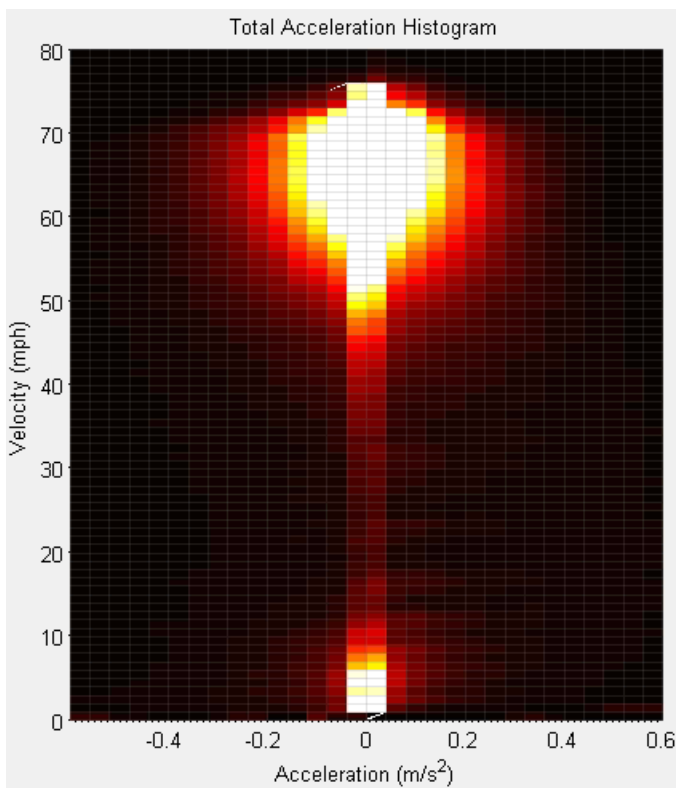

(b) Medium mass

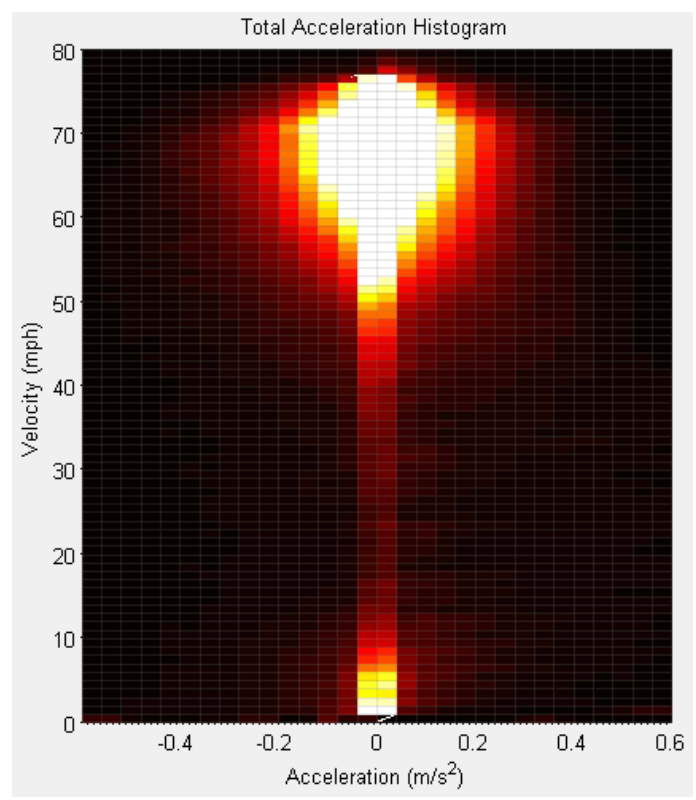

(c) High mass

Figure 30 Comparison of the speed vs. acceleration distributions for the low, medium and high mass operating conditions. 
The procedure described in section 4.2 was again applied to generate a synthetic drive cycle that represents the overall usage for this trucking fleet's operation. The medium mass case, which was the largest data set, was used to develop the synthetic drive cycle. The synthetic drive cycle generation using the medium mass case was started before the comparisons were made for the low and high mass cases, and it would have required significant changes to the data to start over using the complete data set for the synthetic cycle creation. Since resources for the project were limited, it was decided to proceed with the medium mass distribution, since it is so similar to the total usage and included over half of the total driving time.

The base synthetic drive cycle created for the project is shown in Fig. 31. The second by second data for this drive cycle is included in a table in the Appendix. This was generated using the non-zero speed data initially, although some brief stops were added at the beginning and end of the cycle and after the short micro-trip in the first 100 seconds. The total length of this drive cycle is 1997 seconds.

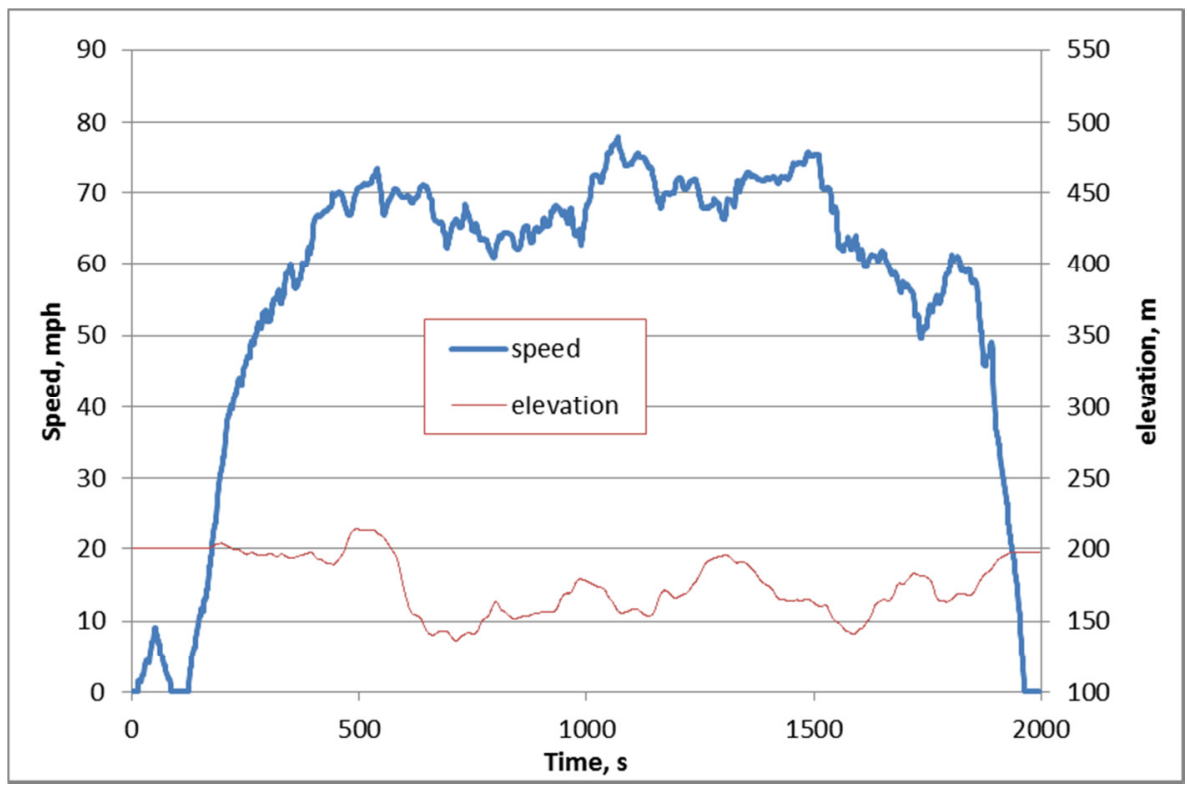

Figure 31 The main synthetic drive cycle developed for the TTEA project, representing the overall usage of the HTDC fleet.

The bivariate speed-acceleration histogram is shown for the HTDC synthetic drive cycle in Fig. 32, along with the total histogram that includes all of the driving data corresponding to the medium mass case. As in the case of the synthetic drive cycle development for the validation case, there are a number of bins in the synthetic cycle histogram representing operating conditions that are not evident in the total histogram representing the original data. These points are a result of using 1-second time intervals when creating the synthetic cycle, as discussed in the previous section. The number of such points in the synthetic histogram for this case is a bit higher than in the case of the validation synthetic drive cycle since the complete set of driving data, with the much larger duration of time represented in the original histogram, contains a more broad set of data in all bins of the histogram. Nonetheless, the overall match to the total histogram is excellent: the error between the synthetic cycle and the original drive cycle distribution, based on a sum of squares metric ( $L 2$ norm) is less than $2 \%$. 
The accelerations and decelerations for the initial speed ramp-up to and final deceleration from highway speeds occur at a significantly lower rate than a truck would normally follow, but this provides the lower level accelerations that are representative of the lower speed range, as discussed previously.
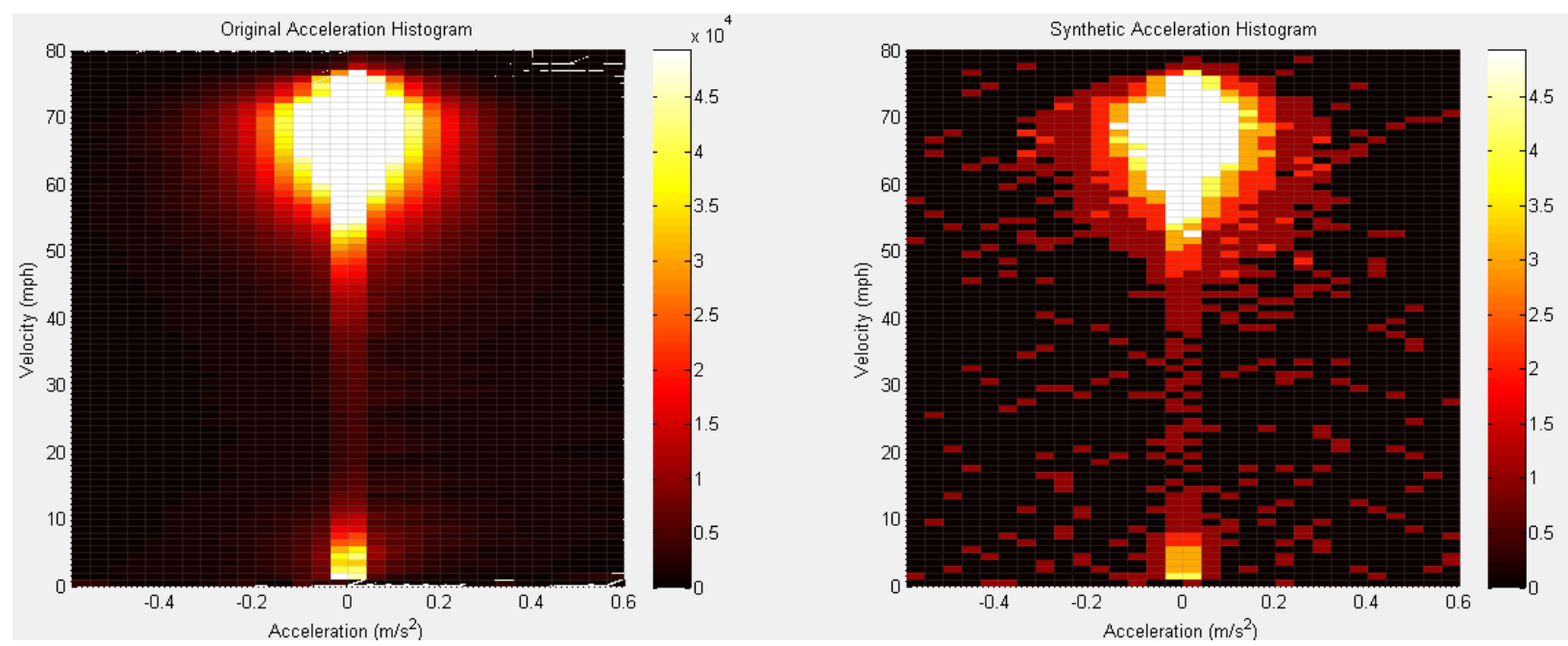

Figure 32 Comparison of the distribution of the synthetic drive cycle and the original histogram containing all of the data from the medium mass operation.

The base synthetic cycle was modified to include the same fraction of idling data as was present in the complete data set. This is handled implicitly (the fraction of idle time is used as an input) in the tractive energy model when evaluating idling energy losses, but for the purpose of presenting a drive cycle with the proper level of idling, representative of the full usage, adding the idling explicitly to the drive cycle is appropriate. A summary of the idling and driving data statistics from all of the measured data is presented in Table 6, and Figure 33 shows the modified synthetic drive cycle with idling present at the average level from all three mass levels. The idling was divided into two types, depending on the duration of each stop. Short idling was defined as having a duration of less than 5 minutes. This would include stops at traffic signals and stop signs, operations in moderate congestion, and short-term stops such as may occur for a rest stop. Long idling was defined to be anything over 5 minutes in duration. This would include stops to pick up or drop off a load, significant traffic congestion or incidents on the highway, and long-term stops (for example, overnight) when the engine is permitted to run to maintain "hoteling" functions. A stop is only considered to be idling if the engine speed is non-zero, and these data only include times when the engine continues to operate. The majority of the long idling periods consisted of multiple hours of continuous stopped operations. It is evident from the table data that this fleet consumed rather significant quantities of fuel due to idling, since the overall fraction of idling time to the total operation time was very nearly $50 \%$. The short idling was only responsible for about $2.6 \%$ of this total, while long idling was responsible for $46.3 \%$ of all engine operation time. 
Table 6: Summary of idling, separated by mass case.

\begin{tabular}{|l|r|r|r|}
\hline & \multicolumn{1}{|c|}{ Low mass } & \multicolumn{1}{|c|}{ Medium mass } & \multicolumn{1}{|c|}{ mass } \\
\hline Total driving time duration, not including stops, sec & $15,493,295$ & $22,745,343$ & $4,834,513$ \\
Short idle durations (<5 minute stops), sec & $1,083,571$ & $1,042,229$ & 215,590 \\
Long idle durations (>5 minutes), sec & $15,968,788$ & $19,107,477$ & $5,527,152$ \\
& & & \\
\% time short idling: & $3.33 \%$ & $2.43 \%$ & $2.04 \%$ \\
\% time long idling: & $49.07 \%$ & $44.54 \%$ & $52.26 \%$ \\
Total \% idle time: & $52.40 \%$ & $46.97 \%$ & $54.29 \%$ \\
Estimated fuel consumption due to idling, L & 8763 & 10,355 & 2951 \\
& & & \\
Avg consumption per distance traveled due to & & & 2.19 \\
idling, L/100 km & 2.14 & 1.64 & 2.11 \\
Long-idle only avg consumption, L/100 km & 2.01 & & \\
\hline
\end{tabular}

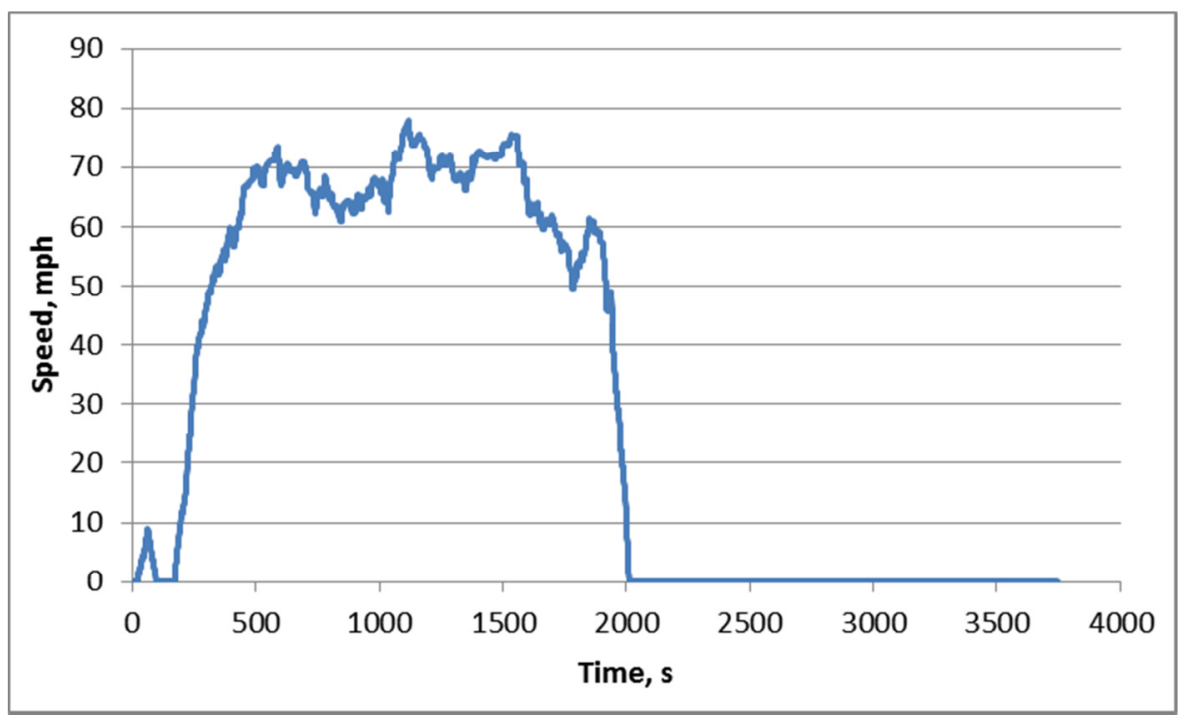

Figure 33 Modified synthetic drive cycle, with the proper ratio of idle time for the overall average. The cycle duration is 3448 seconds.

The total duration of idling for the six trucks was responsible for the consumption of approximately 22,000 liters (5830 gallons) of diesel fuel, or nearly 1000 gallons for each truck. This corresponds to about $4.5 \%$ of the total fuel consumed by the trucks during the year. If auxiliary power units (APUs) were used in the fleet, the fuel savings, assuming a $1.4 \mathrm{~L} /$ hour reduction in idling fuel consumption, would have been $15,790 \mathrm{~L}$ (4172 gallons). This quantity of idling fuel consumption clearly justifies the use of APUs for fleets with such high levels of idling. The short duration idling only corresponded to about $2.7 \%$ of the engine operation time. The fuel savings possible with engine start-stop technology would have been no greater than 1200 liters of fuel for this fleet for the year. While it is a worthwhile goal to minimize idling to the greatest extent possible, this level of fuel savings probably cannot justify the purchase of start-stop engine technology by itself. Nonetheless, this technology could become quite 
common even in heavy duty vehicles in the future, and would reduce fuel consumption by about $0.2 \%$ for this class 8 tractor-trailer application.

\subsection{Tractive energy reductions and fuel savings associated with vehicle efficiency technologies}

We now present the energy savings results calculated with the tractive energy model based on the synthetic drive cycle representing the fleet's overall usage. The results for each of the low, medium and high mass cases are first presented, then these results are combined based on a distance-based weighting of each mass case. The tractive energy model was first run using a mass of $17,755 \mathrm{~kg}$, which is the average mass for the low mass grouping of driving data determined from the analysis presented in section 3.2 (after adjustment). The total driving tractive energy and the contributions from each energy loss factor used in the analysis are show in Table 7. The tractive energy for a reduction in mass is also shown, since this result is used in calculating the mass sensitivity in the tractive energy analysis [2]. The fuel savings estimated for a $2000 \mathrm{~kg}$ mass reduction, a rolling resistance coefficient reduction by 0.0015 $(1.5 \mathrm{~kg} /$ ton $)$, and a reduction in aerodynamic drag by $10 \%$, both with and without regenerative braking, are shown in Fig. 34. These changes in the parameters, used in the tractive energy model are believed to be representative of reductions achievable through vehicle lightweighting, improvements in tire rolling resistance corresponding to a replacement of traditional dual tires with New Generation Wide Base Single (NGWBS) tires, and the use of aerodynamic reduction devices that are available for tractor trailers. The energy reductions achievable with regenerative braking are modeled by considering the total energy consumed by braking during decelerations, and we have assumed an overall efficiency of $80 \%$ for the regenerative braking system. The average predicted fuel economy for the low mass case is $7.32 \mathrm{mpg}$ for the baseline vehicle configuration.

Table 7: Intermediate results of the tractive energy analysis for the low mass case, using the synthetic drive cycle. The results are based on the default model parameters shown in Table 5.

\begin{tabular}{|l|r|r|}
\hline Tractive Energy Contributions & Total Energy (MJ) & Percent of $\mathrm{E}_{\text {trac,drive }}$ \\
\hline $\mathrm{E}_{\text {trac,drive }}$ & 181.24 & -- \\
$\mathrm{E}_{\text {trac,drive }}$ (mass reduced 2000kg) & 171.73 & $94.8 \%$ \\
$\mathrm{E}_{\mathrm{RR}, \text { drive }}$ & 55.16 & $30.4 \%$ \\
$\mathrm{E}_{\mathrm{RR}, \text { brake }}$ & 6.34 & $3.5 \%$ \\
$\mathrm{E}_{\text {aero,drive }}$ & 96.20 & $53.1 \%$ \\
$\mathrm{E}_{\text {aero,brake }}$ & 10.21 & $5.6 \%$ \\
$\mathrm{E}_{\text {brakes }}$ & 13.71 & $7.6 \%$ \\
\hline
\end{tabular}




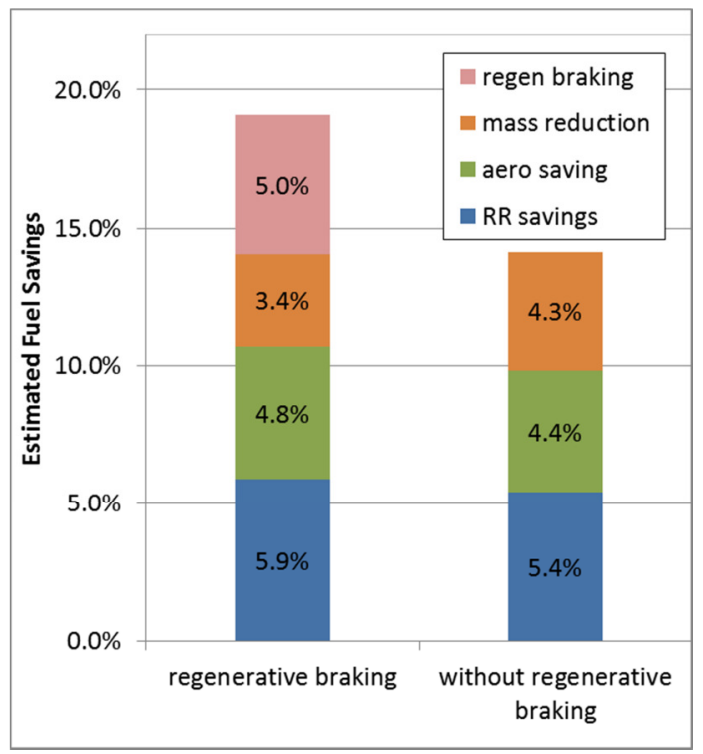

Figure 34 Fuel savings estimate for combinations of advanced efficiency technologies for the low mass case.

The results for the tractive energy contributions and the fuel savings corresponding to vehicle lightweighting, the use of low rolling resistance tires, aerodynamic drag reduction devices, and regenerative braking for the medium mass case are shown in Table 8 and Fig. 35. This is based on a mass of $27,248 \mathrm{~kg}$ used in the tractive energy model, which is the average mass determined in section 3.2 (after adjustment) for the medium mass grouping of driving data. The average predicted fuel economy for this mass case is $6.04 \mathrm{mpg}$ in the baseline vehicle configuration.

Table 8: Predicted driving tractive energy and contributions from energy loss factors for the medium mass case, using the synthetic drive cycle.

\begin{tabular}{|l|r|r|}
\hline Tractive Energy Contributions & Total Energy (MJ) & Percent of $\mathrm{E}_{\text {trac,drive }}$ \\
\hline $\mathrm{E}_{\text {trac,drive }}$ & 227.52 & -- \\
$\mathrm{E}_{\text {trac,drive }}$ (mass reduced 2000kg) & 217.64 & $95.7 \%$ \\
$\mathrm{E}_{\mathrm{RR}, \text { drive }}$ & 81.62 & $35.9 \%$ \\
$\mathrm{E}_{\mathrm{RR}, \text { brake }}$ & 12.77 & $5.6 \%$ \\
$\mathrm{E}_{\text {aero,drive }}$ & 92.68 & $40.7 \%$ \\
$\mathrm{E}_{\text {aero,brake }}$ & 13.73 & $6.0 \%$ \\
$\mathrm{E}_{\text {brakes }}$ & 27.31 & $12.0 \%$ \\
\hline
\end{tabular}




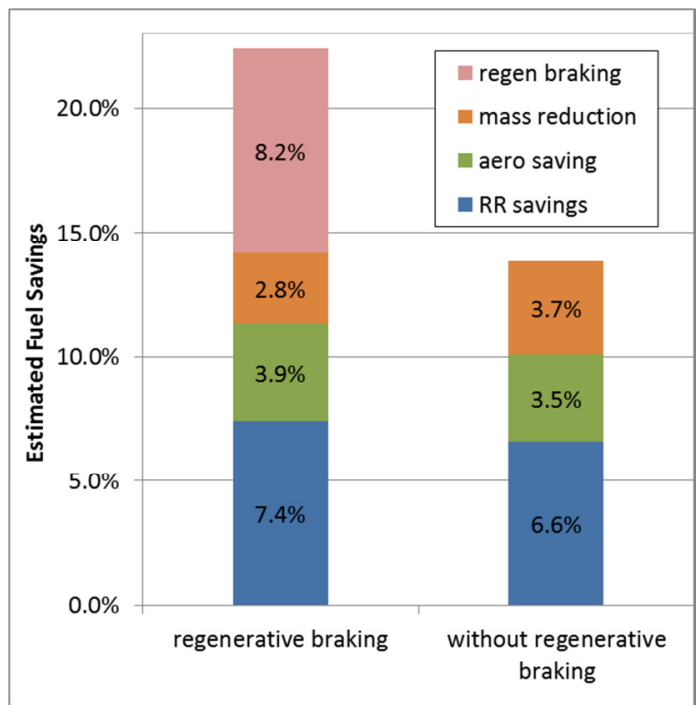

Figure 35 Fuel savings estimate for combinations of advanced efficiency technologies for the medium mass case.

For the high mass case, the results from the tractive energy analysis are presented in Table 9 and Fig. 36. The analysis used a mass of $34,741 \mathrm{~kg}$, corresponding to the average mass determined in section 3.2 (after adjustment) for the high mass grouping of driving data. The baseline configuration had a predicted fuel economy of $5.30 \mathrm{mpg}$ for this mass case.

Table 9: Driving tractive energy and contributions from the different energy loss factors for the high mass case, based on the synthetic drive cycle.

\begin{tabular}{|l|r|r|}
\hline Tractive Energy Contributions & Total Energy (MJ) & Percent of $\mathrm{E}_{\text {trac,drive }}$ \\
\hline $\mathrm{E}_{\text {trac,drive }}$ & 264.96 & -- \\
$\mathrm{E}_{\text {trac,drive }}$ (mass reduced 2000kg) & 254.91 & $96.2 \%$ \\
$\mathrm{E}_{\mathrm{RR}, \text { drive }}$ & 101.64 & $38.4 \%$ \\
$\mathrm{E}_{\mathrm{RR}, \text { brake }}$ & 18.70 & $7.1 \%$ \\
$\mathrm{E}_{\text {aero,drive }}$ & 90.32 & $34.1 \%$ \\
$\mathrm{E}_{\text {aero,brake }}$ & 16.09 & $6.1 \%$ \\
$\mathrm{E}_{\text {brakes }}$ & 38.96 & $14.7 \%$ \\
\hline
\end{tabular}




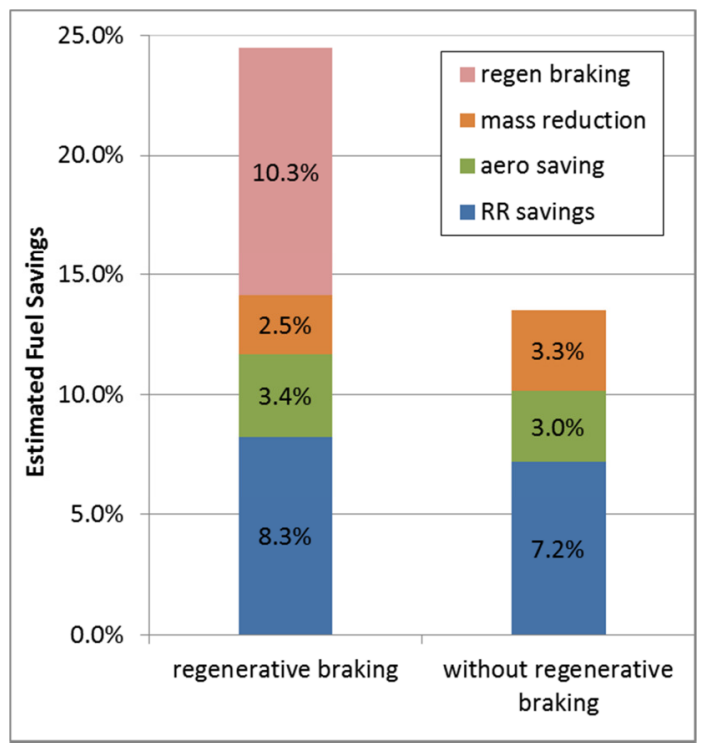

Figure 36 Fuel savings estimate for combinations of advanced efficiency technologies for the high mass case.

These results show that as the mass increases, the contribution to the driving tractive energy from both the tire rolling resistance and braking increase, while the other factors contribute approximately the same to the tractive energy. As a result, the fuel savings from rolling resistance and regenerative braking increase for the higher load conditions, while the relative fuel savings generated by the same aerodynamic improvement or mass reduction are somewhat lower as the mass increases, although the net fuel savings due to these factors will remain very similar at different mass levels. The savings predicted from regenerative braking becomes relatively significant at the higher mass levels, which is somewhat surprising for the predominantly freeway operations that this application experiences.

\subsection{Average fuel savings for the overall fleet usage}

To use the above results from each mass case to quantify the energy savings potential from different vehicle efficiency technologies and combinations of technologies for the overall operation of the fleet, we must appropriately weight the results based on the fraction of distance traveled at each mass level. The following analysis provides a means to use the same analysis methodology used in the tractive energy model for a single run to combine the mass cases in a consistent and appropriate manner.

It is clear that the tractive energy contribution from a given mass case (or another vehicle configuration, to generalize) is proportional to the distance traveled for that configuration, and we can define an average tractive energy per unit distance traveled, which we denote as $E_{\text {trac }}^{\prime}$. This distance-normalized tractive energy factor corresponds to the overall usage (i.e. the drive cycle) corresponding to each given vehicle configuration. For each configuration, $j$, we can therefore express the tractive energy on a perdistance-traveled basis as

$$
E_{\text {trac.j }}=E_{\text {trac }, j}^{\prime} L_{j}
$$


where $L_{j}$ is the distance traveled in configuration $j$. The overall tractive energy requirement due to the combination of all vehicle configurations (i.e. for the full fleet usage) is given by

$$
E_{\text {trac, overall }}=E_{\text {trac, overall }}^{\prime} \sum_{j=1}^{N} L_{j}=\sum_{j=1}^{N} E_{\text {trac }}^{\prime}\left(m_{j}\right) L_{j} .
$$

where $\mathrm{N}$ is the number of configurations and the first sum on the left hand side represents the distance traveled in all of the configurations. We should be cognizant that, by combining tractive energy terms across the different vehicle configurations, we have implicitly assumed that the vehicle parameters used in the tractive energy model that do not influence each $E_{t r a c, j}^{\prime}$ term remain constant. For example, this assumes that a single average engine thermal efficiency is appropriate for all of the configurations considered. While the thermal efficiency, in reality, will vary with the engine load, the effect is not expected to be dramatic with the drive cycle not differing for the three mass cases we are considering. This analysis, is therefore reasonable for our particular scenario, but if very different configurations are evaluated using this approach, one should realize that the assumptions of the model may become less realistic than for a single configuration.

Continuing with our line of reasoning, based on Eq. (8) and Eq. (12), we expect that the distancenormalized tractive energy relationship must hold true both for the total driving tractive energy and for the individual contributions to the driving tractive energy corresponding to each energy loss factor (driving and braking contributions from rolling resistance and aerodynamic drag, as well as the contribution from the braking tractive energy). Combining the two equations, we can therefore decompose the contributions associated with the various technologies using the following relationship:

$$
E_{t r a c, i}^{\prime}=\frac{\sum_{j=1}^{N} E_{t r a c, i}^{\prime}\left(m_{j}\right) L_{j}}{\sum_{j=1}^{N} L_{j}}=\sum_{j=1}^{N} E_{t r a c, i}^{\prime}\left(m_{j}\right) w_{j},
$$

where $i$ represents each energy loss factor and $w_{j}$, the mass weighting factor, is given by the ratio of the distance traveled at each mass to the total distance traveled. Using Eq. (13), the results for each energy loss factor calculated for each configuration can be converted to obtain the average tractive energy loss factors associated with the overall fleet operation. Table 10 shows the set of distance-normalized loss factors (and the mass sensitivity) for the different mass cases and that determined using Eq. (13) for the combined vehicle usage. The values for $\Delta E_{\text {trac,drive,mass }}^{\prime}$ and $\Delta E_{\text {trac,braking,mass }}^{\prime}$ represent the change in the driving and braking tractive energies, respectively, associated with a mass reduction of $2000 \mathrm{~kg}$. For the calculation, the distance traveled in each mass case is shown in Table 11. 
Table 10: Distance-normalized tractive energy factors from the tractive energy analysis for each mass case, and the combined result for the full fleet.

\begin{tabular}{|l|cccccccc|}
\hline & $\mathrm{E}_{\text {trac,drive }}^{\prime}$ & $\mathrm{E}_{\mathrm{RR}, \text { drive }}^{\prime}$ & $\mathrm{E}_{\mathrm{RR}, \text { brake }}^{\prime}$ & $\mathrm{E}_{\text {aero,drive }}^{\prime}$ & $\mathrm{E}_{\text {aero,brake }}^{\prime}$ & $\mathrm{E}_{\text {braking }}^{\prime}$ & $\Delta \mathrm{E}_{\text {trac,drive,mass }}^{\prime}$ & $\Delta \mathrm{E}_{\text {trac, braking,mass }}$ \\
\hline Low mass & 3.601 & 1.094 & 0.126 & 1.907 & 0.202 & 0.272 & -0.189 & -0.052 \\
Medium mass & 4.522 & 1.618 & 0.253 & 1.837 & 0.272 & 0.541 & -0.197 & -0.059 \\
High mass & 5.268 & 2.015 & 0.371 & 1.791 & 0.319 & 0.772 & -0.200 & -0.063 \\
\hline \hline Combined & 4.284 & 1.479 & 0.222 & 1.857 & 0.253 & 0.473 & -0.194 & -0.057 \\
\hline
\end{tabular}

Table 11: Distance traveled in each mass case.

\begin{tabular}{|l|r|r|}
\hline & \multicolumn{1}{|c|}{$\begin{array}{c}\text { distance } \\
\text { traveled }(\mathrm{km})\end{array}$} & $\begin{array}{c}\text { average } \\
\text { mass }(\mathrm{kg})\end{array}$ \\
\hline Low mass & 389,240 & 17,755 \\
Medium mass & 594,307 & 27,248 \\
High mass & 126,636 & 34,741 \\
\hline \hline Combined & $1,110,182$ & 24,774 \\
\hline
\end{tabular}

With the combined tractive energy terms, the sensitivity factors for the tractive energy model were calculated for the overall vehicle usage and the final fuel savings estimates associated with the vehicle efficiency technologies considered were determined. The contribution from each energy loss factor for the overall combined vehicle usage is shown in Table 12, and the fuel savings estimates corresponding to our standard parameter variations were determined for the complete fleet usage. These fuel savings estimates are shown in Fig. 37.

Table 12: The contributions to the driving tractive energy from each energy loss factor for the overall, combined fleet usage, based on the synthetic drive cycle.

\begin{tabular}{|l|r|r|}
\hline Tractive Energy Contributions & Total Energy (MJ) & Percent of $\mathrm{E}_{\text {trac,drive }}$ \\
\hline$E_{\text {trac,drive }}$ & 216.10 & -- \\
$E_{\text {trac,drive }}$ (mass reduced 2000kg) & 206.29 & $95.5 \%$ \\
$E_{R R, \text { drive }}$ & 74.63 & $34.5 \%$ \\
$E_{R R, \text { brake }}$ & 11.19 & $5.2 \%$ \\
$E_{\text {aero,drive }}$ & 93.65 & $43.3 \%$ \\
$E_{\text {aero,brake }}$ & 12.77 & $5.9 \%$ \\
$E_{\text {brakes }}$ & 23.87 & $11.0 \%$ \\
\hline
\end{tabular}




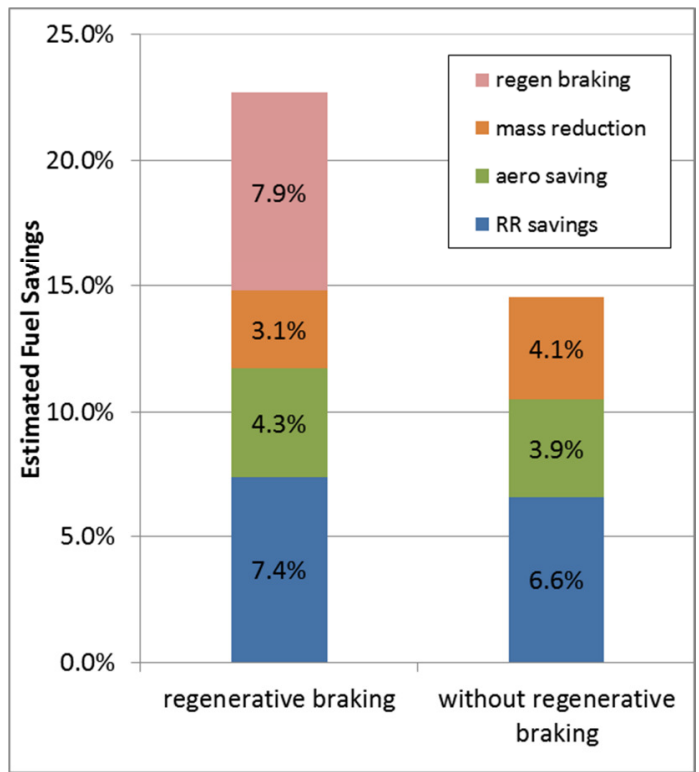

Figure 37 Fuel savings estimate for combinations of advanced efficiency technologies for the combined usage in the HTDC fleet.

\subsection{Summary of the tractive energy model results for the class 8 tractor- trailer application evaluated}

The savings in fuel consumption predicted by the tractive energy model for this class 8 tractor-trailer fleet are rather impressive for the technologies considered. By implementing the rolling resistance and aerodynamic drag technologies, which can be done as retrofits to existing vehicles, over a $10 \%$ improvement in fuel economy can be achieved, based on the assumptions for the vehicle parameter changes. The reduction in the rolling resistance coefficient by 0.0015 , or $1.5 \mathrm{~kg} / \mathrm{ton}$, used in this analysis depends on the initial and final set of tires used on the vehicle, but this level of reduction is very typical, if not on the conservative side, when replacing typical dual tires with NGWBS tires. Similarly, the $10 \%$ reduction in aerodynamic drag coefficient used in the analysis seems to be rather typical based on results that have been reported in the literature for fuel efficiency gains with aerodynamic drag reduction devices. Vehicle lightweighting, while it represents a change in vehicle design that must be implemented for new vehicles, can also yield quite significant fuel savings, and research and development of lighter materials and manufacturing methods that can reduce truck mass should be pursued by vehicle manufacturers and the transportation research community. The predicted benefits of the use of a regenerative braking system in this tractor-trailer application are quite impressive. For the overall usage of this fleet, regenerative braking is predicted to reduce fuel consumption by nearly $8 \%$, and if low rolling resistance tires and aerodynamic drag reductions are used the same time, the regenerative braking increases the benefits by an additional 1.2\%. As shown in Fig. 36, if a fleet operates at higher average mass levels, the hybrid savings can exceed $10 \%$. This fuel savings potential suggests that further study of hybridization of class 8 tractor-trailers should be pursued. 


\subsection{Consideration of the accuracy of using a substitute drive cycle as opposed to the synthetic drive cycle}

The synthetic drive cycle approach was developed to accurately characterize the usage of the fleet, and the validation of this method shows that the fuel savings estimates are very representative of what can be achieved in the fleet when implementing advanced vehicle efficiency technologies. As an alternative approach, we selected a single drive cycle for which the vehicle mass was constant that had a usage as similar as possible to the overall usage of the fleet. Histograms of individual drive cycles were compared to the total histogram representing the complete fleet usage, and a measured drive cycle that visually similar to the total histogram was selected. The tractive energy analysis was then run using this "substitute" measured drive cycle. Since many drive cycles used for fuel efficiency evaluations are selected based on short-term driving measurements without careful evaluation of the overall usage, it seems reasonable to consider the errors in following such an approach. This comparison is intended to shed some light on how accurate one might expect fuel efficiency predictions to be when arbitrarily selected drive cycles are used to represent a particular usage.

The drive cycle selected for this evaluation was from the medium mass set of data. A comparison of the acceleration-velocity bivariate histogram is compared to that of the total histogram for the medium mass case in Fig. 38.
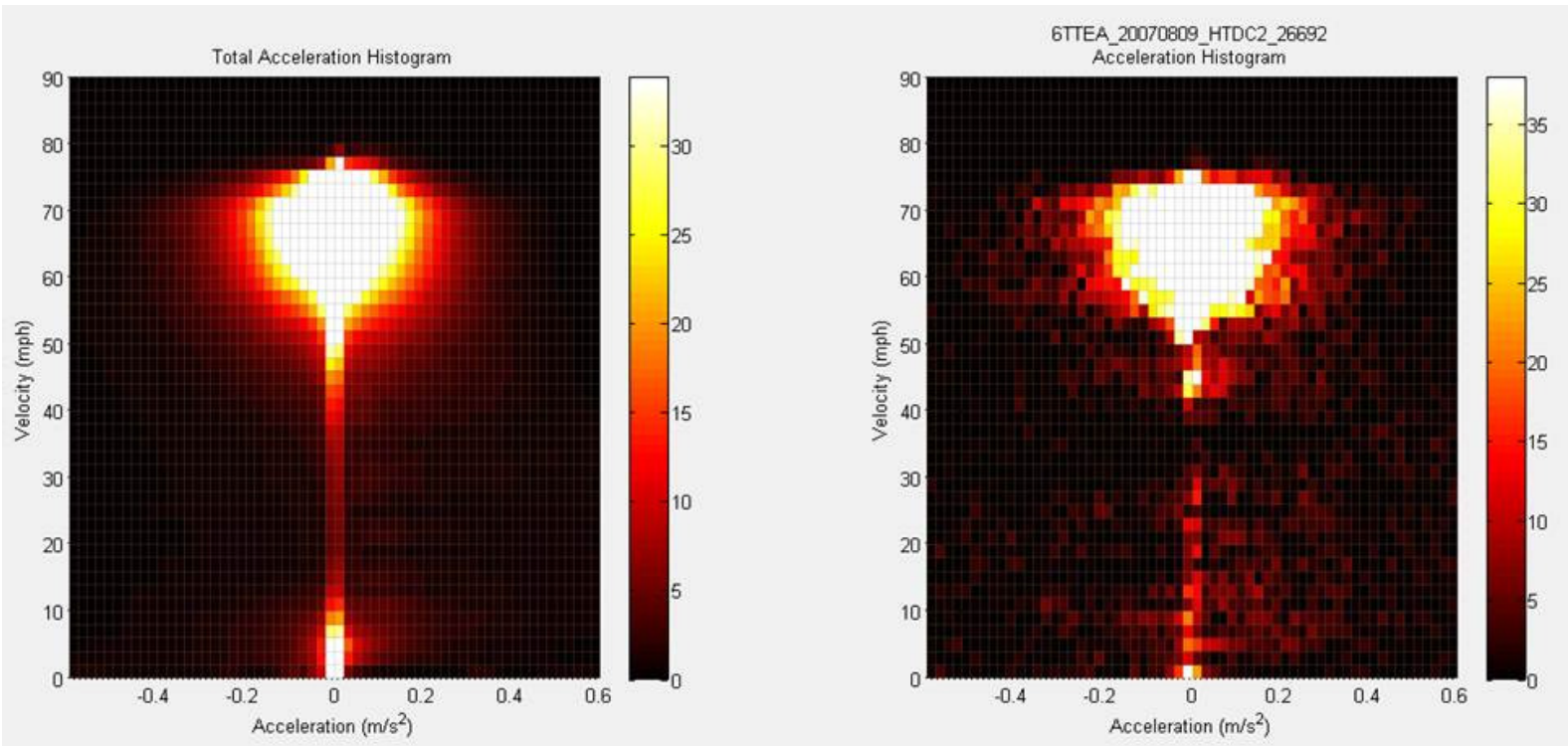

Figure 38 Comparison of the histogram for substitute measured drive cycle to that of the overall usage for the HTDC fleet.

The substitute cycle matched the shape and highway speeds and accelerations quite well. When comparing individual days of driving, it is fairly common to have portions of travel that are not characteristic of the overall usage, for example extended driving at a lower speed or missing some range of accelerations in the highway operation so that the individual day's histogram does not appear to be similar to the total histogram. The single-day drive cycle was processed in the same way as all other driving data used in this study prior to running the tractive energy model. The same cycle was also used 
in the MOVES model emissions analysis (see section 6 of this report), and the idling duration was adjusted to have the same percentage of idling as the overall usage. The same mass value and all other input parameters used in the medium synthetic cycle analysis were also used in the tractive energy analysis for the substitute synthetic cycle so that the comparisons would be on the same basis.

Table 13 and Fig. 39 show the results of the tractive energy analysis. Comparing the percent contributions to the driving tractive energy in Table 13 to those for the medium mass synthetic cycle result (Table 8, result is repeated in the last column of Table 13) shows clearly that there are large differences between the characteristics of the synthetic cycle and the substitute drive cycle.

Table 13: The contributions to the driving tractive energy from each energy loss factor for the overall, combined fleet usage, based on the synthetic drive cycle.

\begin{tabular}{|l|r|r|r|}
\hline Tractive Energy Contributions & $\begin{array}{c}\text { Total Energy } \\
(\mathrm{MJ})\end{array}$ & $\begin{array}{c}\text { Percent of } \mathrm{E}_{\text {trac,drive, }} \\
\text { substitute measured } \\
\text { cycle }\end{array}$ & $\begin{array}{c}\text { Percent of } \mathrm{E}_{\text {trac,drive }} \\
\text { medium mass } \\
\text { synthetic cycle }\end{array}$ \\
\hline$E_{\text {trac,drive }}$ & 2566.32 & -- & -- \\
$E_{\text {trac,drive }}$ (mass reduced 2000kg) & 2436.66 & $94.9 \%$ & $95.5 \%$ \\
$E_{R R, \text { drive }}$ & 726.29 & $28.3 \%$ & $34.5 \%$ \\
$E_{R R, b r a k e}$ & 238.92 & $9.3 \%$ & $5.2 \%$ \\
$E_{\text {aero,drive }}$ & 793.85 & $30.9 \%$ & $43.3 \%$ \\
$E_{\text {aero,brake }}$ & 276.67 & $10.8 \%$ & $5.9 \%$ \\
$E_{\text {brakes }}$ & 506.68 & $19.7 \%$ & $11.0 \%$ \\
\hline
\end{tabular}

The percentage contribution to the driving tractive energy due to vehicle braking is nearly twice as great for the substitute cycle, while the driving tractive energy contributions from both rolling resistance and aerodynamic drag are considerably lower. Comparing the fuel savings estimate with Fig. 35 shows that there is a sizable difference in this result, also. In particular, the predicted savings from regenerative braking is considerably greater for the substitute drive cycle, but differences of 0.7 to $1.3 \%$ for each of the no regenerative braking results also exist.

This comparison shows that large errors in the predicted fuel savings can occur if the usage is not well represented by the drive cycle used for fuel efficiency evaluations. Even when the usage, as evidenced by the acceleration-velocity histogram, appears to match fairly well, relatively large differences can occur. To ensure that a selected drive cycle is representative of the actual usage of a particular application, it is rather important that a statistical evaluation of the usage be conducted. 


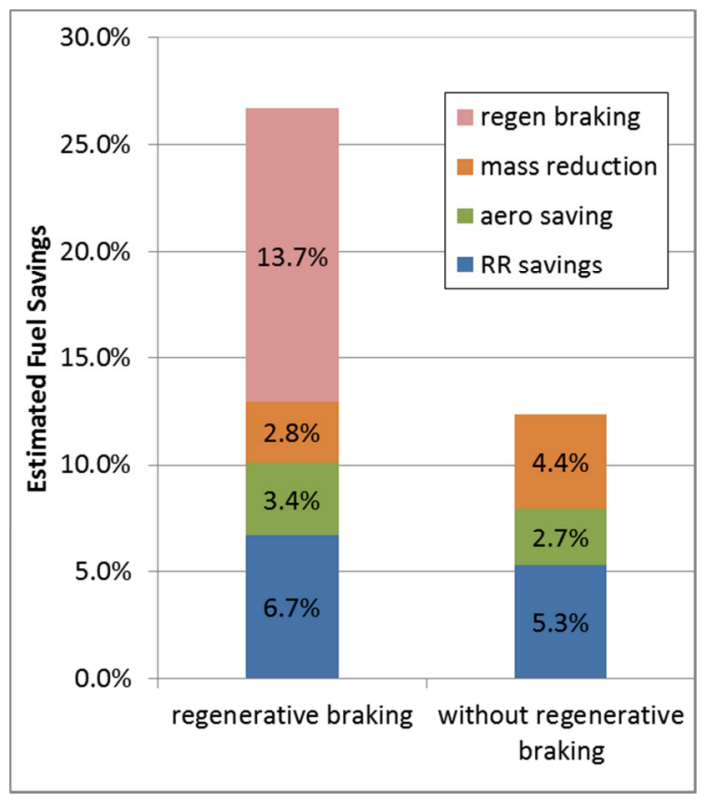

Figure 39 Fuel savings estimate using the substitute measured drive cycle.

\section{EPA MOVES (Motor Vehicle Emission Simulator) Model Analysis}

The Motor Vehicle Emissions Simulator (MOVES) is the U.S. Environmental Protection Agency's (EPA) current regulatory computer model used for estimating on-road emissions from cars, trucks, motorcycles, and buses. It incorporates the latest emission measurement data and more sophisticated calculation algorithms than MOBILE, the previous vehicle emissions regulatory model. Transient driving conditions that influence the instantaneous engine load, such as vehicle acceleration, cruising and coasting, road grade, and aerodynamic and rolling resistances have been shown to influence emissions [8-10], and for that reason, these vehicle activities or parameters have been integrated into MOVES using the vehicle specific power (VSP) approach [11].

The official release of MOVES2010 occurred on March 2, 2010. (The numbers next to the acronym refer to the version number of the model.) At the present, the two latest versions are MOVES2010a released in September 2010, and MOVES2010b released in April 2012 which includes a number of improvements over the former. However, the net impact of the changes on emissions between versions 2010a and $2010 \mathrm{~b}$ is very small at both the County and Project level scales. The MOVES web site [12] is the source for the software, technical and user manuals, and other guidance documents.

In MOVES, emission rates are calculated using operating mode distributions. Operating mode is defined in terms of classes or ranges of VSP and vehicle road speed that have distinct emission rates associated with them. For heavy duty (HD) vehicles, scaled tractive power (STP), which will be defined later in the report, is used instead of VSP. The operating mode distribution is the fraction of time the vehicle operates (or carries out its behavior) within a definite range of VSP (or STP) and vehicle speeds, which are referred to as bins. A bin is similar to the location in an array or matrix that will be treated later as an algebraic entity for further calculations. The operating modes of most concern for HD vehicles are 
shown in Table 14. For example, operating mode bin no. 14 is defined as Cruise/Acceleration, $6 \mathrm{~kW}<=$ STP $<9$ kW; $1 \mathrm{mph}<=$ Speed $<25 \mathrm{mph}$.

Table 14: MOVES operating modes

\begin{tabular}{|c|c|}
\hline opModelD & opModeName \\
\hline 0 & Braking \\
\hline 1 & Idling \\
\hline 11 & Low Speed Coasting; STP $<0 ; 1<=$ Speed $<25$ \\
\hline 12 & Cruise/Acceleration; $0<=\mathrm{STP}<3 ; 1<=$ Speed $<25$ \\
\hline 13 & Cruise/Acceleration; $3<=S T P<6 ; 1<=$ Speed $<25$ \\
\hline 14 & Cruise/Acceleration; $6<=$ STP $<9 ; 1<=$ Speed $<25$ \\
\hline 15 & Cruise/Acceleration; $9<=\mathrm{STP}<12 ; 1<=$ Speed $<25$ \\
\hline 16 & Cruise/Acceleration; $12<=S T P ; 1<=$ Speed $<25$ \\
\hline 21 & Moderate Speed Coasting; STP $<0 ; 25<=$ Speed $<50$ \\
\hline 22 & Cruise/Acceleration; $0<=\mathrm{STP}<3 ; 25<=$ Speed $<50$ \\
\hline 23 & Cruise/Acceleration; $3<=\mathrm{STP}<6 ; 25<=$ Speed $<50$ \\
\hline 24 & Cruise/Acceleration; $6<=\mathrm{STP}<9 ; 25<=$ Speed $<50$ \\
\hline 25 & Cruise/Acceleration; $9<=\mathrm{STP}<12 ; 25<=$ Speed $<50$ \\
\hline 26 & Cruise/Acceleration; $12<=$ STP; $25<=$ Speed $<50$ \\
\hline 27 & Cruise/Acceleration; $12<=S T P<18 ; 25<=$ Speed $<50$ \\
\hline 28 & Cruise/Acceleration; $18<=S T P<24 ; 25<=$ Speed $<50$ \\
\hline 29 & Cruise/Acceleration; $24<=S T P<30 ; 25<=$ Speed $<50$ \\
\hline 30 & Cruise/Acceleration; $30<=$ STP; $25<=$ Speed $<50$ \\
\hline 33 & Cruise/Acceleration; STP $<6 ; 50<=$ Speed \\
\hline 35 & Cruise/Acceleration; $6<=\mathrm{STP}<12 ; 50<=$ Speed \\
\hline 36 & Cruise/Acceleration; $12<=$ STP; $50<=$ Speed \\
\hline 37 & Cruise/Acceleration; $12<=\mathrm{STP}<18 ; 50<=$ Speed \\
\hline 38 & Cruise/Acceleration; $18<=\mathrm{STP}<24 ; 50<=$ Speed \\
\hline 39 & Cruise/Acceleration; $24<=\mathrm{STP}<30 ; 50<=$ Speed \\
\hline 40 & Cruise/Acceleration; $30<=S T P ; 50<=S p e e d$ \\
\hline
\end{tabular}

Note: this table was extracted from the MySQL "operatingMode" table and was edited for clarity

A priority hierarchy is used by the model to estimate emissions at the Project level scale. It can utilize (1) the default drive schedules built into MOVES, (2) a user-supplied drive cycle, or (3) a user supplied operating mode distribution. First preference is given to a user-supplied operating mode distribution, followed by a user-supplied drive cycle, and lastly to applying built-in default schedules that require user-supplied average speed information. In the end, to estimate emissions MOVES always relies on the operating mode distribution; however, if the user only supplied average speed information, the model will apply this in conjunction with the default driving schedules to create a generalized operating mode distribution.

Only 12 default driving schedules currently exist in MOVES for HD vehicles; each has an average speed associated with it [13]. A description of these driving schedules is shown in Table 15. For representing a 
real world driving condition, this approach is incomplete. On the other hand, a user-supplied drive cycle that contains second-by-second speed and road grade information from which the model can then generate a more realistic operating mode distribution is a significant improvement over the average speed approach.

Table 15: MOVES default driving schedules for HD vehicles

\begin{tabular}{r|rr}
\hline driveScheduleID & averageSpeed (mph) & driveScheduleName \\
\hline \hline 301 & 5.8 & HD 5mph Non-Freeway \\
302 & 11.2 & HD 10mph Non-Freeway \\
303 & 15.6 & HD 15mph Non-Freeway \\
304 & 19.4 & HD 20mph Non-Freeway \\
305 & 25.6 & HD 25mph Non-Freeway \\
306 & 32.5 & HD 30mph Non-Freeway \\
351 & 34.3 & HD 30mph Freeway \\
352 & 47.1 & HD 40mph Freeway \\
353 & 54.2 & HD 50mph Freeway \\
354 & 59.4 & HD 60mph Freeway \\
355 & 71.7 & HD High Speed Freeway \\
399 & 25.3 & HD Freeway Ramp \\
\hline
\end{tabular}

Note: this table was extracted from the MySQL "drivesSchedule" table and was edited for simplicity

MOVES is capable of quantifying the reduction of some exhaust emissions by the use of certain technologies available for diesel buses and trucks that have been retrofitted with emission control equipment. However, this is generally used for state implementation plans (STP) and/or transportation conformity analyses at the local county level in non-attainment area. (This is an area considered to have air quality worse than the EPA's National Ambient Air Quality Standards, and the local or state governing agency for the area must devise a plan to meet the standard or risk losing some forms of federal financial assistance.) A retrofit is defined broadly to include any technology that when applied to an existing diesel engine will achieve emission reductions beyond that currently required by EPA regulations at the time the engine was certified [14]. Trailer aerodynamic kits and low rolling resistance tires can be considered examples of aftermarket retrofits [15]. But for SIPs in non-attainment areas, correction factors are simply applied to vehicle fleet populations to show reduction in emissions due to the percentage effectiveness of the retrofit. Thus, advanced efficiency technologies to improve fuel economy, such as aerodynamic drag reduction devices, low rolling resistance tires, and lightweight materials are not easily represented directly in MOVES. However, the default parameters that the model uses for STP calculations can be edited or altered in the MySQL database. For the most part these default parameters in MOVES are based on overall fleet averages derived from historic data, but they are defined in terms of road load coefficients, tire rolling resistance, aerodynamic drag, and friction losses in the drivetrain. MOVES is written in JavaTM and uses the MySQL relational database management system. The principal user inputs and outputs, and the internal working storage locations necessary for MOVES to run are located in the MYSQL database. 
The MOVES2010a model, hereafter referred to simply as MOVES, was used to estimate exhaust emissions using the drive cycles of class 8 tractor-trailers based on real-world usage. The emissions evaluated with these simulations were carbon monoxide $(\mathrm{CO})$, nitrogen oxides $\left(\mathrm{NO}_{\mathrm{X}}\right)$, gaseous hydrocarbons $(\mathrm{HC})$, primary $\mathrm{PM}_{10}$ and $\mathrm{PM}_{2.5}$. The summation of nitrogen oxide (NO) and nitrogen dioxide $\left(\mathrm{NO}_{2}\right)$ is $\mathrm{NO}_{x}$. Particulate matter less than 10 microns or less than 2.5-microns in aerodynamic diameter are $\mathrm{PM}_{10}$ or $\mathrm{PM}_{2.5}$, respectively which denote the size of the particles. Total $\mathrm{PM}$ here refers to the summation of organic carbon (OC) and elemental carbon (EC) derived from running exhaust only. Sulfate particulates from the exhaust, brake and tire wear were not modeled. MOVES was also used to estimate energy consumption which is a surrogate measure for fuel usage and/or carbon dioxide $\left(\mathrm{CO}_{2}\right)$ emissions.

\subsection{Objective}

The University of Tennessee, Knoxville (UTK) performed all of the MOVES modeling and analysis for this project. This phase of the study focused on truck drive cycle impacts on running exhaust emissions. Real world (measured) drive cycles and synthetic (i.e., condensed or abbreviated) drive cycles were compared to understand the drive cycle effects on emissions and to validate the use of the synthetic cycle as a replacement for the original driving data in emissions calculations.

The following analyses using MOVES were performed in the study: (1) second-by-second emission estimates were compared with measured emissions from a dynamometer test performed using a measured drive cycle; (2) MOVES model emission estimates based on measured drive cycles from Oak Ridge National Laboratory's (ORNL) heavy truck duty cycle (HTDC) data set were compared with emission estimates from representative synthetic drive cycles; (3) emissions benefits of advanced efficiency technologies such as aerodynamic drag reduction devices, low rolling resistance tires, and lightweight materials for class 8 long-haul trucks were assessed using MOVES; and (4) results obtained using the default drive cycles in MOVES were compared with model results from simulations using a measured, representative truck drive cycle for this application as well as with the synthetic drive cycle developed for the project.

\subsection{MOVES Modeling Analysis/Discussion}

\subsubsection{MOVES mean base rates for running exhaust emissions}

The MOVES mean base rates are average emission rates for pollutants in each combination of regulatory class, model year group, fuel type, pollutant process, and operating mode bin combination. The mean base rates are adjusted by ambient temperature and humidity and by air conditioning (AC) correction factors. The mean base running exhaust emissions are stored in the "emissionRateByAge" table in the MOVES input MYSQL database file. All simulations using the MOVES model in this study were performed using mean base rates for a model year of 2005 and an age of 0-3 years of HD trucks to compare with the tested emission results on the ORNL test cycle. These are the inputs that are relevant for the trucks tested in the Heavy Truck Duty Cycle (HTDC) project at the time that all measurements 
were performed. It should be noted that the same vehicle model year and age were applied to all vehicle drive cycle data used in the MOVES phase of this study.

\subsubsection{Emissions calculation procedure using the MOVES method}

In MOVES, the scaled tractive power (STP) parameter is used for estimating HD vehicle emissions. STP was designed to fit into the existing operating mode framework which was developed originally for lightduty vehicles [16]. Road load coefficients, including tire rolling resistance, aerodynamic drag, and friction losses in the drivetrain are incorporated into the STP term using these default place holder coefficients (the rolling A term, the rotating B term and the drag $C$ term) which will be defined later, and are located in the "sourceUseType" table in the MySQL database. The default source mass for the vehicle category is 31.4038 tonne $(69,234 \mathrm{lb})$; this is based on other historic data that were collected for this vehicle category and represents an average vehicle mass for the category.

The general steps to determine running exhaust emissions for HD diesel trucks in MOVES are the following:

1. The scaled tractive power (STP) in units of $\mathrm{kW}$ is calculated for each second using the general equation

$$
\operatorname{STP}_{\mathrm{t}}=\frac{A v_{t}+B v_{t}^{2}+C v_{t}^{3}+m v_{t}\left(a_{t}+g \sin \theta\right)}{f_{s c a l e}}
$$

where

$$
\begin{aligned}
& v=\text { velocity }(\mathrm{m} / \mathrm{s}) \\
& a=\text { acceleration, }\left(\mathrm{m} / \mathrm{s}^{2}\right) \\
& m=\text { mass, (tonne) } \\
& t=\text { time, }(\mathrm{s}) \\
& g=\text { acceleration due to gravity, }\left(9.8 \mathrm{~m} / \mathrm{s}^{2}\right) \\
& \theta_{t}=\text { road grade, (radians) } \\
& A=\text { rolling resistance, }(\mathrm{kW}-\mathrm{s} / \mathrm{m}) \\
& B=\text { rotating resistance, }\left(\mathrm{kW}-[\mathrm{s} / \mathrm{m}]^{2}\right) \\
& C=\text { aerodynamic drag, }\left(\mathrm{kW}-[\mathrm{s} / \mathrm{m}]^{3}\right) \\
& f_{\text {scale }}=\text { scaling or fixed mass factor (default value }=17.1 \text { ) }
\end{aligned}
$$

The velocity is the actual second-by-second speed data. Acceleration is calculated from velocity as $\left(v_{t}-v_{(t+1)}\right) /(1 \mathrm{~s})$. (All data used in the analysis used 1-second time intervals.)

For Combination long-haul trucks (i.e., heavy duty diesel vehicles see default values):

Rolling A term $=\mu_{0} \cdot g \cdot m$, with default value $=2.08126 \mathrm{~kW}-\mathrm{s} / \mathrm{m}$

Rotating B term $=\mu_{1} \cdot g \cdot \mathrm{m}$, default value $=0 \mathrm{~kW}-\mathrm{s}^{2} / \mathrm{m}^{2}$

Drag $C$ term $=\left(C_{d} \cdot \rho_{\text {air }} \cdot R\right) / 2+\mu_{2} \cdot g \cdot m$, default value $=0.0041884 \mathrm{~kW}-\mathrm{s}^{3} / \mathrm{m}^{3}$ 
where

$\mu_{0}=\left(C_{R R}\right)$ zero-order tire rolling-resistance coefficient, (unitless)

$\mu_{1}=$ first-order tire rolling-resistance coefficient, $(\mathrm{s} / \mathrm{m})$

$\mu_{2}=$ second-order tire rolling-resistance coefficient, $\left(\mathrm{s}^{2} / \mathrm{m}^{2}\right)$

$C_{d}=$ aerodynamic drag coefficient of the vehicle, (unitless)

$R=$ cross-sectional frontal area of the vehicle, $\left(\mathrm{m}^{2}\right)$

$\rho_{\text {air }}=$ density of air, $\left(1.202 \mathrm{~kg} / \mathrm{m}^{3}\right)$

2. The operating mode for each second is then determined using the $\operatorname{STP}_{t}$ value.

3. Finally, using the operating mode and the source type/vehicle model year-age, the mean base running exhaust emissions are selected from the "emissionratebyage" table in the MOVES input database file for the regulatory class, the model year, and the age group using diesel fuel.

4. Temperature and humidity were not adjusted in this study.

\subsubsection{Comparison of MOVES predictions with measured emissions data from one of the HTDC test trucks}

Data from the West Virginia University (WVU) test sequence no. 5271 using the test drive cycle ORNL4LS (presented in a previous section of this report) were processed to create second-by-second emissions estimates using the MOVES mean base rates, vehicle speed, and road grade profiles. The ORNL4LS drive cycle is shown in Fig. 40. The WVU data were derived from chassis dynamometer measurements and exhaust emission sampling. Three repeated emission tests were conducted for this cycle during December 2007. The tests were performed on one of the test vehicles from the HTDC project, a Volvo tractor that had a model year 2005 Cummins ISX $475 \mathrm{hp}$ diesel engine. It had a standard exhaust system that was not fitted with a particulate trap or a catalytic converter. 


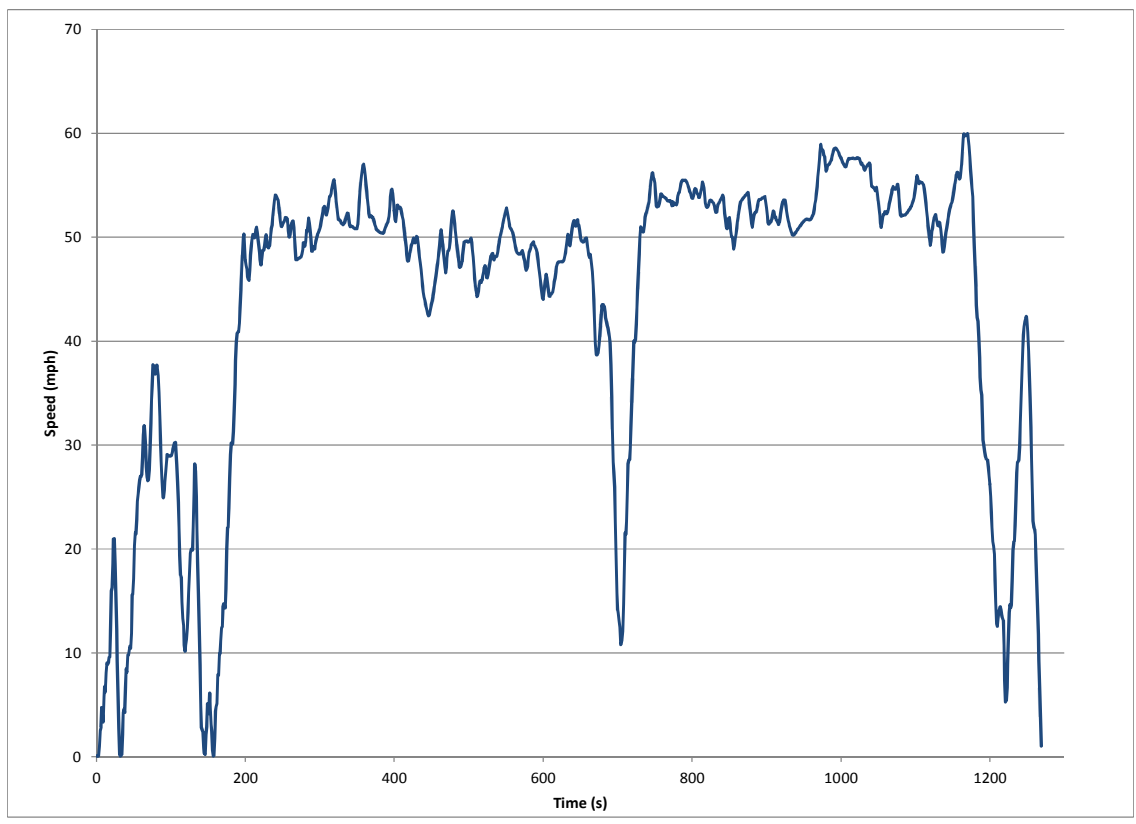

Figure 40 Measured dynamometer hub speed data from the WVU dynamometer testing with the ORNL4LS drive cycle.

Figure 41 is a 10-minute segment showing second-by-second $\mathrm{NO}_{\mathrm{x}}$ emissions as measured during the dynamometer testing conducted at West Virginia University and this is compared with the NOx emissions predicted by MOVES for the same drive cycle. Predictions for $\mathrm{CO}$ and $\mathrm{HC}$ emissions, and the fuel rate using the ECU data are shown in Figures 42 through 44, respectively. 


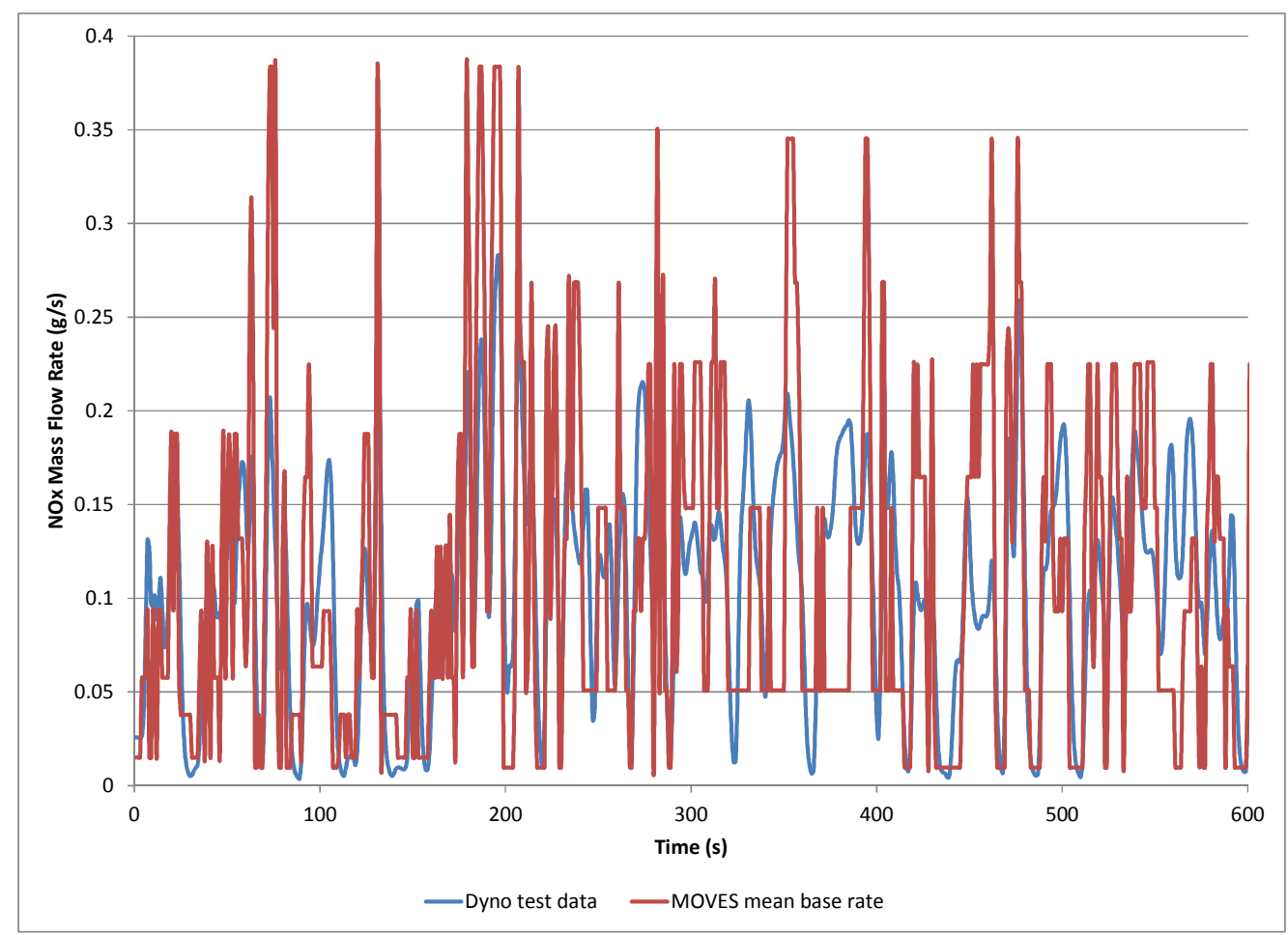

Figure 41 Nitrogen oxide emissions measured during the dynamometer test and the mean base rates predicted by MOVES using speed data from the ECU.

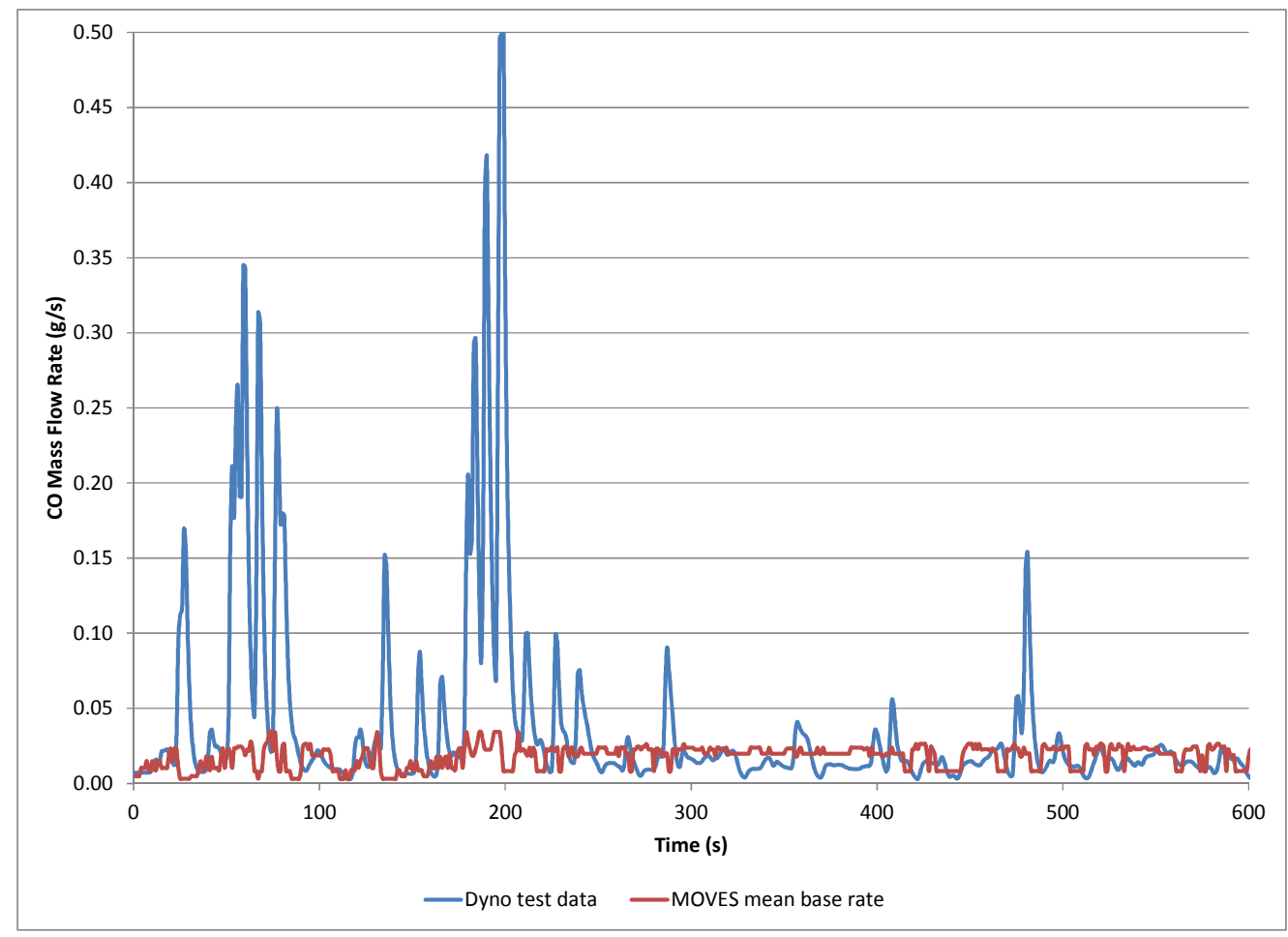

Figure 42 Carbon monoxide emissions measured during the dynamometer test and the mean base rates predicted by MOVES using speed data from the ECU. 


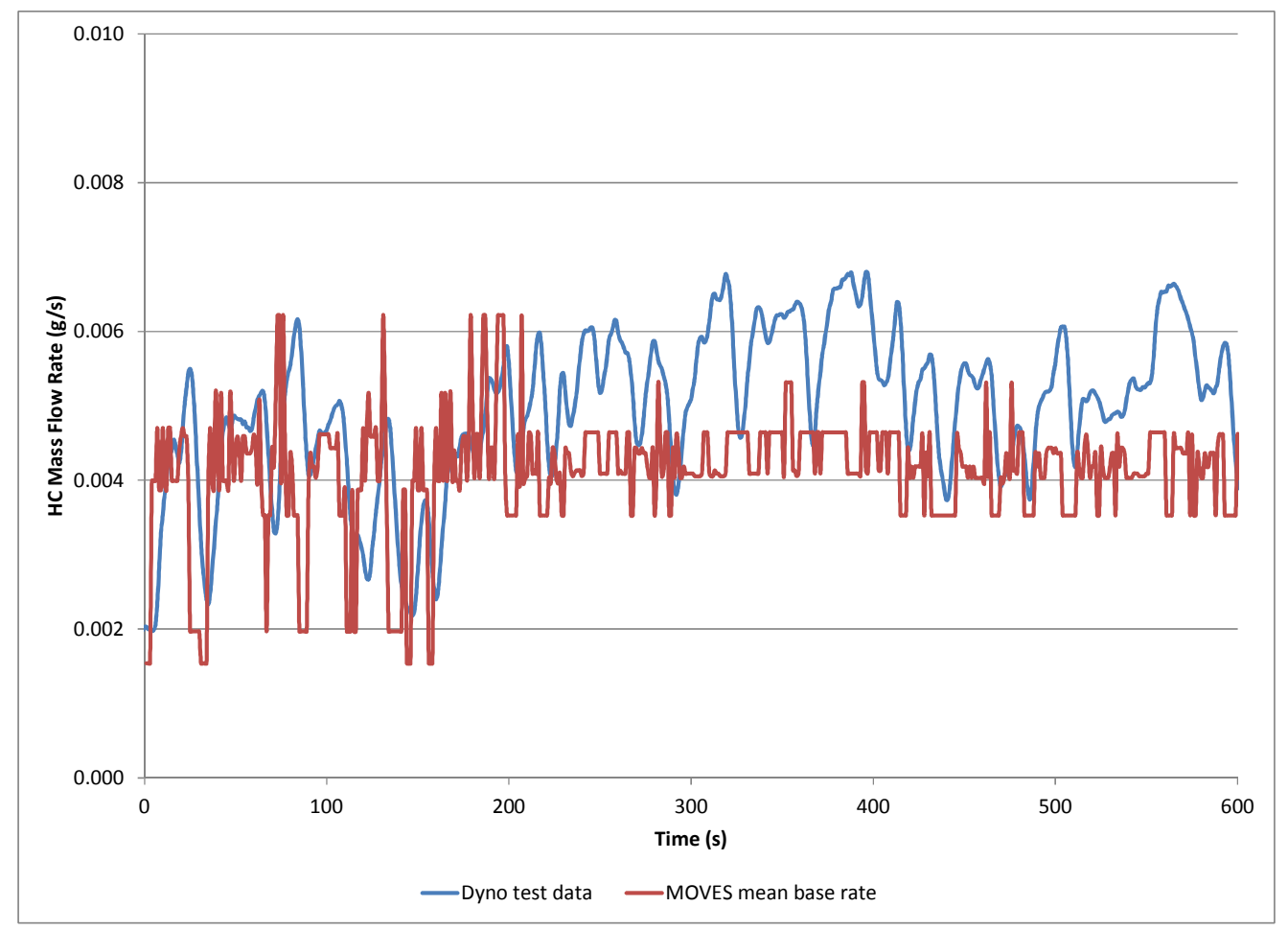

Figure 43 Gaseous hydrocarbon emissions measured during the dynamometer test and the mean base rates predicted by MOVES using speed data from the ECU.

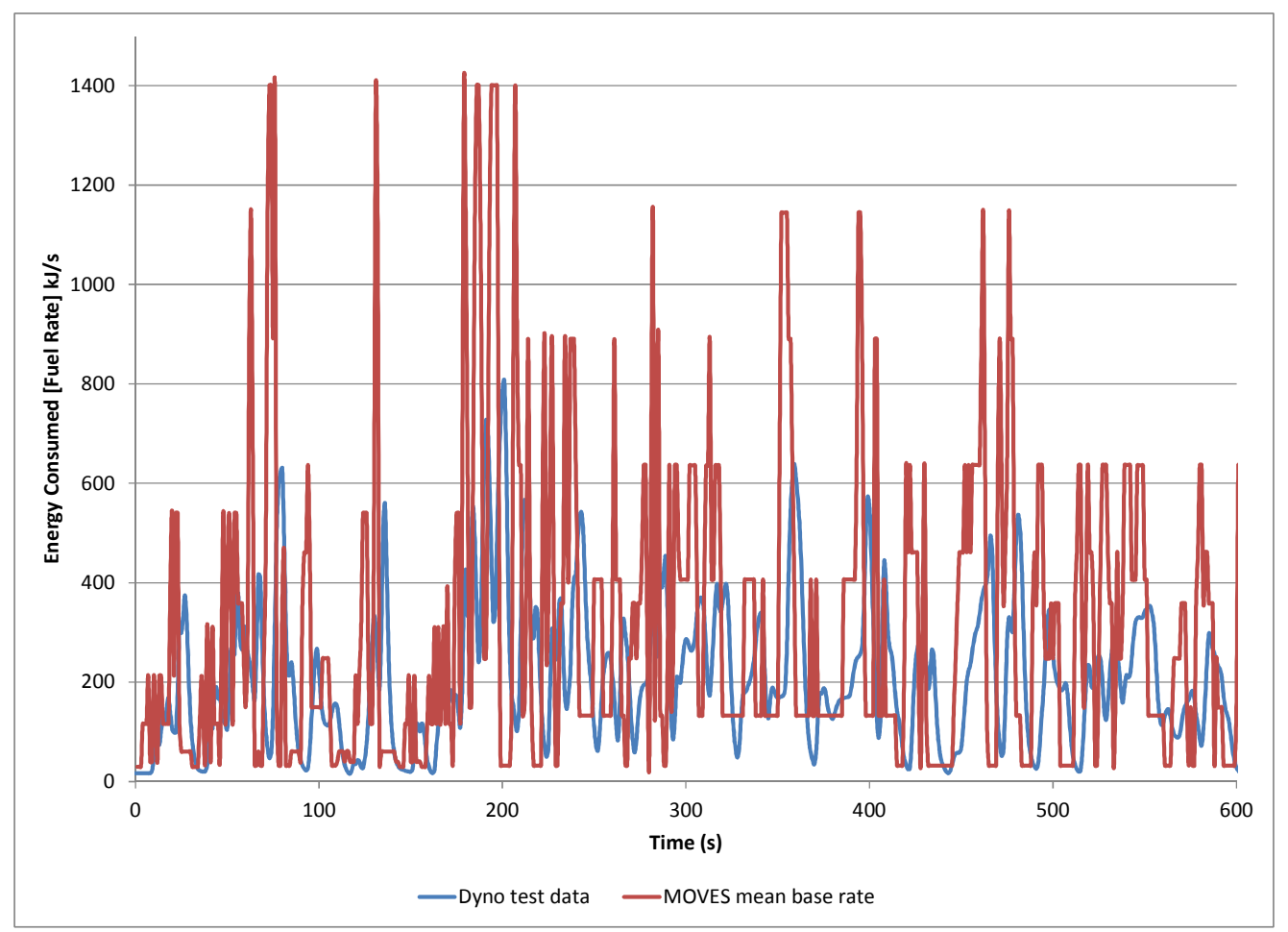

Figure 44 Energy consumption measured during the dynamometer test and the mean base rates predicted by MOVES using speed data from the ECU. 
It is evident from the $\mathrm{CO}$ and $\mathrm{HC}$ predictions that the MOVES model still under-estimated the emissions during some parts of the drive cycle. The CO prediction, during periods of heavy acceleration (for example, from around 175-200 s), does not match the magnitude of the measured CO emission. Although the MOVES model does significantly under-predict the $\mathrm{CO}$ emissions that were measured for this vehicle, the absolute level of these emissions are extremely low, and the levels during relatively steady speeds are reasonably well-predicted. Since MOVES uses an emissions map derived from steady state emissions data for its predictions, some transient operating conditions are not well predicted. Also, the data used in the emissions maps for the model are based on measurements from multiple vehicles and are not representative of any single truck. The periods where higher spikes in the $\mathrm{CO}$ were measured took place when the vehicle was accelerating at near-maximum levels, which for vehicles with exhaust gas recirculation (EGR) generally leads to less lean conditions that tend to generate high CO levels. This is a known weakness of the MOVES model (and other map-based emission prediction models), and a more detailed analysis tool capable of modeling the transient engine operating conditions would be needed to accurately predict such transient emission variations. Emission maps are research and development tools that allow characterization of fuel consumption and emissions at a given time on the basis of vehicle operating parameters, including engine speed and torque, etc. Given the very low level of $\mathrm{CO}$ emissions, this is not felt to be a cause for concern. Similarly, for the HC emissions, the errors between the measured and predicted emissions, while large on a relative basis for this particular vehicle, are relatively small in consideration of the absolute emission levels.

The discrepancies observed between the model and test results were communicated to the MOVES team at EPA for their comment. The consensus was that the model had been developed for the purpose of estimating overall fleet emissions and should not be expected to match the second-by-second emissions of a single truck. That is, the MOVES emission rates reflect a fleet average, and the modal emission rates are meant to model aggregate emissions over different driving patterns. As a result, the modeling team at EPA was not surprised by the emission rate differences between a single truck and MOVES. Nonetheless, the EPA did note that they were re-evaluating the HD emission rates in this model year range based on their evaluation of new data recently collected from several trucks in the field.

Total emissions were compared between dynamometer measurements and MOVES data. However, instead of using the default MOVES vehicle parameters described above, the following values were applied: coefficient of drag $\left(C_{d}=0.58\right)$, frontal area $\left(R=10 \mathrm{~m}^{2}\right)$, rolling resistance coefficients $\left(\mu_{0}\left[=C_{R R}\right]\right.$ $=0.007, \mu_{1}=0$ and $\left.\mu_{2}=0.0\right)$, and gross vehicle weight ( $m=18.71$ tonne or $41,248 \mathrm{lb}$ ). These were used because they are more representative of the actual test vehicle's configuration. Thus, the rolling, rotating, and drag terms for the STP equation were $A=1.28351 \mathrm{~kW}-\mathrm{s} / \mathrm{m}, B=0$, and $C=0.00427162 \mathrm{~kW}$ $\mathrm{s}^{3} / \mathrm{m}^{3}$, respectively.

Table 16 summarizes energy consumption and total emissions from the dynamometer test and MOVES. These results revealed that MOVES tended to under-predict $\mathrm{NO}_{x}, \mathrm{CO}$, and $\mathrm{HC}$ emissions and over-predict energy consumption relative to the dynamometer test. However, since the aggregated emission values for the dynamometer test and MOVES are comparable, it adds support to the previous comment from EPA that MOVES can model fleet averages but it cannot match the second-by-second emissions of a single truck. 
Table 16: Comparison of total emissions and energy consumption results from dynamometer measurements and MOVES predictions.

\begin{tabular}{r||ccc}
\hline \multicolumn{1}{l||}{ Emission/Energy Results } & $\begin{array}{c}\text { dynamometer } \\
\text { test result }\end{array}$ & MOVES & $\begin{array}{c}\text { \% Difference } \\
\text { (Dyno-MOVES)/Dyno }\end{array}$ \\
\hline \hline $\mathrm{NO}$ (g) & 139 & 111 & $20.2 \%$ \\
$\mathrm{HC}(\mathrm{g})$ & 43 & 23 & $45.1 \%$ \\
Energy consumption (kJ) & 6.9 & 5.3 & $24.0 \%$ \\
\hline
\end{tabular}

\subsubsection{Validation of the synthetic cycle methodology for MOVES simulations: comparison of results using a synthetic drive cycle that represents a full day of measured drive cycle data}

To evaluate the accuracy of using a synthetic drive cycle to represent large sets of driving data, a comparison was made between MOVES predictions using a single day's drive cycle data and a much shorter synthetic drive cycle developed to represent the day of driving. This was done to first validate the procedure used to generate the synthetic drive cycle. The original drive cycle was from a typical 8 hour freight hauling episode. The synthetic drive cycle was developed by ORNL using the DCGen tool (see section 4 of this report for more details). The vehicle speed and road grade profiles for the measured drive cycle, as well as the synthetic drive cycle were used in MOVES. These two cycles were provided by ORNL also for comparative study to test technologies associated with aerodynamic drag reduction, low rolling resistance tires, and lightweight materials for trucks.

The original drive cycle, which was measured in August 2007, included a total travel distance for the route of approximately $860 \mathrm{~km}$ (535 miles). It included about $2 \%$ engine idling time where vehicle speed was less than $1.6 \mathrm{~km} / \mathrm{h}$ (i.e., below $1 \mathrm{mph}$ ), which is based on the MOVES idling definition. The synthetic cycle is 1646 second in duration with approximately $48.5 \mathrm{~km}$ (30.1 mi) of travel distance and about $0.3 \%$ of idling time. For both cycles, instead of using the default MOVES vehicle parameter values, the parameters corresponding to the vehicle configuration and actual load during the measured drive cycle were used. These parameters are coefficient of drag $\left(C_{d}=0.58\right)$, frontal area $\left(R=10 \mathrm{~m}^{2}\right)$, rolling resistance coefficients $\left(\mu_{0}\left[=C_{R R}\right]=0.007, \mu_{1}=0 \& \mu_{2}=\sim 0.0\right)$, and gross vehicle weight $(m=18.71$ tonne or $41,250 \mathrm{lb}$ ). Thus, the rolling, rotating and drag terms in the STP equation were $A=1.28351 \mathrm{~kW}-\mathrm{s} / \mathrm{m}, \mathrm{B}$ $=0$, and $C=0.00427162 \mathrm{~kW}-\mathrm{s}^{3} / \mathrm{m}^{3}$, respectively.

Emissions were modeled using (1) the default vehicle parameters listed above, (2) a mass reduction of $2,000 \mathrm{~kg}$ with the remaining default parameters, $(3)$ a rolling resistance reduction $\left(C_{R R}=0.0055\right)$ with the remaining default parameters, $(4)$ an aerodynamic drag reduction $\left(C_{d}=0.52\right)$ with the remaining default parameters, (5) both rolling resistance and aerodynamic drag reductions with the default parameter of mass only, and (6) all three vehicle parameters are reduced (i.e., mass, rolling resistance and 
aerodynamic drag). The purpose of these comparisons was to quantify the emissions benefit associated with the use of advanced efficiency technologies on the truck, and to quantify the accuracy of using the synthetic drive cycle approach in the calculation.

The comparisons between the original drive cycle and the synthetic drive cycle validation case are shown in Table 17. The first column in the table shows the metrics evaluated (the various predicted emissions and the energy consumption). The comparisons are made between all of the runs evaluated for each metric. The second column shows the parameter variations that were used for each run. These are either the default parameters (defined above) to represent the baseline vehicle configuration, or variations relative to the baseline case corresponding to a change in mass, rolling resistance, and/or aerodynamic drag coefficients. These parameter changes were applied one at a time and then in combination to evaluate how the parameter changes (corresponding to different efficiency technologies) affected the emissions rates. All of the cases with the parameter variations were compared to the default parameter case, which is highlighted in the table for each metric considered. The third column contains the emission rates or fuel energy consumption predicted by the model for the full day's drive cycle, and the corresponding rates using the synthetic cycle are listed in the fifth column. For each of the parameter variation cases considered, the percent difference in the emission rates and fuel energy consumption, relative to those calculated for the default parameter run, are listed in the fourth column for the original drive cycle. These data correspond to the emissions reduction (or increase, in the case of a positive percentage change) that the model predicts when the vehicle parameters are reduced (e.g. by implementing appropriate technology modifications to the vehicle). The corresponding difference, relative to the default parameter case, calculated by using the synthetic drive cycle is shown in the sixth column of the table. The last column shows the difference between the percent reduction calculated using the synthetic drive cycle and the reduction calculated with the original drive cycle. This provides an indication of how similar the emissions/energy savings prediction is when using the original drive cycle vs. the synthetic cycle. 
Table 17: Summary of MOVES predicted emissions for the validation synthetic cycle case, with a comparison of the original and synthetic drive cycle results.

\begin{tabular}{|c|c|c|c|c|c|c|}
\hline Metric & Description & $\begin{array}{l}\text { MOVES } \\
\text { result with } \\
\text { the original } \\
\text { drive cycle }\end{array}$ & $\begin{array}{c}\text { \% Difference } \\
\text { due to } \\
\text { parameter } \\
\text { variation(s) } \\
\text { (original cycle) }\end{array}$ & $\begin{array}{l}\text { MOVES } \\
\text { result with } \\
\text { the synthetic } \\
\text { validation } \\
\text { drive cycle }\end{array}$ & $\begin{array}{c}\text { \% Difference } \\
\text { due to } \\
\text { parameter } \\
\text { variation(s) } \\
\text { (synthetic cycle) }\end{array}$ & $\begin{array}{c}\text { Difference } \\
\text { between } \\
\text { \%original \& } \\
\text { \%synthetic } \\
\text { [syn-original] }\end{array}$ \\
\hline \multirow{6}{*}{$\begin{array}{c}\mathrm{NO}_{\mathrm{x}} \\
(\mathrm{g} / \mathrm{mi})\end{array}$} & Default parameters (baseline vehicle configuration) & 7.461 & & 7.561 & & \\
\hline & Change in mass $(2,000 \mathrm{~kg}$ reduction $)$ & 7.446 & $-0.2 \%$ & 7.569 & $0.1 \%$ & $0.3 \%$ \\
\hline & Change in $C_{R R}=0.0055$ & 7.127 & $-4.5 \%$ & 7.226 & $-4.4 \%$ & $0.1 \%$ \\
\hline & Change in $C_{d}=0.52$ & 7.069 & $-5.3 \%$ & 7.194 & $-4.9 \%$ & $0.4 \%$ \\
\hline & Change in $C_{R R}=0.0055 \& C_{d}=0.52$ & 6.713 & $-10.0 \%$ & 6.908 & $-8.6 \%$ & $1.4 \%$ \\
\hline & Change in mass $(2,000 \mathrm{~kg}$ reduction $) \& C_{R R}=0.0055 \& C_{d}=0.52$ & 6.671 & $-10.6 \%$ & 6.869 & $-9.2 \%$ & $1.4 \%$ \\
\hline \multirow{6}{*}{$\begin{array}{c}\mathrm{CO} \\
(\mathrm{g} / \mathrm{mi})\end{array}$} & Default parameters (baseline vehicle configuration) & 1.181 & & 1.197 & & \\
\hline & Change in mass $(2,000 \mathrm{~kg}$ reduction $)$ & 1.188 & $0.6 \%$ & 1.203 & $0.5 \%$ & $-0.1 \%$ \\
\hline & Change in $C_{R R}=0.0055$ & 1.176 & $-0.4 \%$ & 1.189 & $-0.7 \%$ & $-0.3 \%$ \\
\hline & Change in $C_{d}=0.52$ & 1.176 & $-0.4 \%$ & 1.188 & $-0.8 \%$ & $-0.4 \%$ \\
\hline & Change in $C_{R R}=0.0055 \& C_{d}=0.52$ & 1.169 & $-1.0 \%$ & 1.183 & $-1.2 \%$ & $-0.2 \%$ \\
\hline & Change in mass $\left(2,000 \mathrm{~kg}\right.$ reduction) $\& C_{R R}=0.0055 \& C_{d}=0.52$ & 1.174 & $-0.6 \%$ & 1.187 & $-0.8 \%$ & $-0.2 \%$ \\
\hline \multirow{6}{*}{$\begin{array}{c}\mathrm{HC} \\
(\mathrm{g} / \mathrm{mi})\end{array}$} & Default parameters (baseline vehicle configuration) & 0.224 & & 0.227 & & \\
\hline & Change in mass $(2,000 \mathrm{~kg}$ reduction) & 0.224 & $0.0 \%$ & 0.227 & $0.0 \%$ & $0.0 \%$ \\
\hline & Change in $C_{R R}=0.0055$ & 0.225 & $0.4 \%$ & 0.229 & $0.9 \%$ & $0.5 \%$ \\
\hline & Change in $C_{d}=0.52$ & 0.226 & $0.9 \%$ & 0.229 & $0.9 \%$ & $0.0 \%$ \\
\hline & Change in $C_{R R}=0.0055 \& C_{d}=0.52$ & 0.227 & $1.3 \%$ & 0.230 & $1.3 \%$ & $0.0 \%$ \\
\hline & Change in mass $\left(2,000 \mathrm{~kg}\right.$ reduction) $\& C_{R R}=0.0055 \& C_{d}=0.52$ & 0.227 & $1.3 \%$ & 0.230 & $1.3 \%$ & $0.0 \%$ \\
\hline \multirow{6}{*}{$\begin{array}{c}\mathrm{PM}_{2.5} \\
(\mathrm{OC}+\mathrm{EC}) \\
(\mathrm{g} / \mathrm{mi})\end{array}$} & Default parameters (baseline vehicle configuration) & 0.303 & & 0.311 & & \\
\hline & Change in mass $(2,000 \mathrm{~kg}$ reduction $)$ & 0.301 & $-0.7 \%$ & 0.309 & $-0.6 \%$ & $0.1 \%$ \\
\hline & Change in $C_{R R}=0.0055$ & 0.292 & $-3.6 \%$ & 0.300 & $-3.5 \%$ & $0.1 \%$ \\
\hline & Change in $C_{d}=0.52$ & 0.290 & $-4.3 \%$ & 0.299 & $-3.9 \%$ & $0.4 \%$ \\
\hline & Change in $C_{R R}=0.0055$ \& $C_{d}=0.52$ & 0.279 & $-7.9 \%$ & 0.290 & $-6.8 \%$ & $1.1 \%$ \\
\hline & Change in mass $\left(2,000 \mathrm{~kg}\right.$ reduction) $\& C_{R R}=0.0055 \& C_{d}=0.52$ & 0.277 & $-8.6 \%$ & 0.288 & $-7.4 \%$ & $1.2 \%$ \\
\hline \multirow{6}{*}{$\begin{array}{c}\text { Total } \\
\text { energy } \\
\text { consumed } \\
(\mathrm{kJ} / \mathrm{mi})\end{array}$} & Default parameters (baseline vehicle configuration) & 20,587 & & 20,840 & & \\
\hline & Change in mass $(2,000 \mathrm{~kg}$ reduction $)$ & 20,488 & $-0.5 \%$ & 20,821 & $-0.1 \%$ & $0.4 \%$ \\
\hline & Change in $C_{R R}=0.0055$ & 19,598 & $-4.8 \%$ & 19,870 & $-4.7 \%$ & $0.1 \%$ \\
\hline & Change in $C_{d}=0.52$ & 19,427 & $-5.6 \%$ & 19,777 & $-5.1 \%$ & $0.5 \%$ \\
\hline & Change in $C_{R R}=0.0055 \& C_{d}=0.52$ & 18,396 & $-10.6 \%$ & 18,952 & $-9.1 \%$ & $1.5 \%$ \\
\hline & Change in mass $(2,000 \mathrm{~kg}$ reduction $) \& C_{R R}=0.0055 \& C_{d}=0.52$ & 18,253 & $-11.3 \%$ & 18,808 & $-9.8 \%$ & $1.5 \%$ \\
\hline
\end{tabular}

Notes: Emissions are based on mean base rate (w/out temperature \& humidity adjustments); default parameters: $\mathrm{m}=18,710 \mathrm{~kg} ; \mathrm{C}_{\mathrm{RR}}=0.007 ; \mathrm{C}_{\mathrm{d}}=0.58 ;$ frontal area $10 \mathrm{~m}$. chg = changed parameter; def = default parameter; syn = synthetic cycle 
Using $\mathrm{NO}_{\mathrm{x}}$ as an example, the emission rate for the original drive cycle modeled with the default parameters was $7.46 \mathrm{~g} / \mathrm{mile}$, while the corresponding result for the synthetic validation drive cycle is $7.56 \mathrm{~g} / \mathrm{mile}$. The results obtained with the original drive cycle and using the synthetic drive cycle are within just a few percent, and this level of consistency between the two drive cycles for the overall prediction is observed for all of the metrics evaluated. This shows excellent agreement between the results from the two drive cycles and validates that the synthetic cycle is highly representative of the original drive cycle. It is the change in emissions that occur when the vehicle configuration is modified that is most critical for our purposes, however, and we consider these differences in detail. When the mass is reduced by $2,000 \mathrm{~kg}$, the $\mathrm{NO}_{\mathrm{x}}$ emission rate was $7.45 \mathrm{~g} /$ mile for the original drive cycle, and it was $7.57 \mathrm{~g} /$ mile for the synthetic drive cycle. The percent difference between the changed parameter case and the default parameter results is $-0.2 \%$ for the original drive cycle and $0.1 \%$ for the synthetic cycle. A negative difference means that a reduction in emissions occurred for the change from the default to the changed parameter case, while a positive percentage indicates that an increase occurred as a result of the change in parameters. In this case, the difference is extremely small, and even though the direction of change predicted by the two drive cycles are opposite in sign for this case, both cycles result in a predicted change that is near zero and the difference between the two predictions for the emission reduction associated with a $2000 \mathrm{~kg}$ mass reduction is only $0.3 \%$. As seen in the table, the results when using the original drive cycle and those from the synthetic drive cycle are all very close to one another. The difference in the emissions/fuel consumption variation calculated using the synthetic vs. the original drive cycle for any of the metrics is within $1.5 \%$, and in most cases this difference is only a fraction of a percent. The greatest differences appearing in the last column occur when the predicted reductions are the greatest, so the relative error in the predicted emissions reduction for these cases does not become extremely large. Results from both drive cycles show the general trends for the emissions reductions to be quite similar, although some of the predicted emission reductions due to the combinations of technologies are estimated to be lower when using the synthetic drive cycle. Based on these results, we conclude that the synthetic drive cycle provides results that are in excellent agreement with those obtained from the original drive data. This close agreement between the emissions reductions predicted with the original and the synthetic drive cycle indicates that we can reliably use a synthetic drive cycle in the EPA MOVES model to predict the emissions benefits achievable when specific changes in vehicle configuration are applied for a given vehicle usage. These results validate the methodology of using a synthetic drive cycle representing a larger set of drive cycle data to estimate the benefits that can be achieved with particular vehicle configuration changes using MOVES.

\subsubsection{Evaluation of the emissions reductions from advanced efficiency technologies for the overall HTDC fleet usage using MOVES}

As described in section 4 of this report, a synthetic drive cycle that is representative of the overall usage for the HTDC test fleet was developed based on a detailed statistical evaluation of all of the measured drive cycle data from the HTDC project. This synthetic drive cycle was initially developed to represent the medium mass case, but it was found that this usage was very similar to that for all of the mass cases considered, and the same synthetic drive cycle was used to evaluate the fleet's overall usage. As opposed to the synthetic validation cycle considered in the previous section for a single day's worth of driving, the overall usage synthetic drive cycle represents over one million kilometers of travel during 
more than 20,000 hours of operation, and it would not be possible to perform the MOVES analysis using the complete data set in a reasonable period of time. However, we validated that the results obtained by running MOVES using a carefully constructed synthetic drive cycle having a similar statistical distribution of speeds, accelerations and elevation variations yields the same result as would be obtained using the complete data set. The synthetic drive cycle representing the full fleet usage is 3610 seconds in duration with approximately $50.4 \mathrm{~km}$ (31.3 mi) of travel distance; it also includes about $47 \%$ of idling time, which is the same percentage experienced in the actual fleet for the medium mass case.

For the MOVES analysis using the overall usage synthetic drive cycle, the baseline vehicle parameters for the mass, rolling resistance, and aerodynamic drag were selected to correspond as closely as possible to the configuration of the real vehicles tested. As in the previous section, these will be denoted as the default parameters: coefficient of drag $\left(C_{d}=0.62\right)$, frontal area $\left(R=6.5 \mathrm{~m}^{2}\right)$, rolling resistance coefficients $\left(\mu_{0}\left[=C_{R R}\right]=0.007, \mu_{1}=0 \& \mu_{2}=0.0\right)$, and gross vehicle weight ( $m=24.187$ tonne or 53,323 lb). Thus, the rolling, rotating, and drag terms in the STP equation for the baseline vehicle configuration were $A=1.6592 \mathrm{~kW}-\mathrm{s} / \mathrm{m}, \mathrm{B}=0$, and $\mathrm{C}=0.00343788 \mathrm{~kW}-\mathrm{s}^{3} / \mathrm{m}^{3}$, respectively. A primary objective of this study was to conduct an assessment of the emissions reductions that could be realized in the fleet if combinations of various vehicle efficiency technologies are employed. As in the previous analyses, we evaluated the emissions benefits based on implementation of the following technologies: (1) vehicle light-weighting as represented by a vehicle mass reduction of $2,000 \mathrm{~kg},(2)$ the use of low rolling resistance tires, based on a change in the coefficient of rolling resistance, $C_{R R}$, from a value of 0.007 to 0.0055 , and (3) aerodynamic drag reduction, as could be achieved through the use of trailer skirts, more streamlined tractor and trailer designs or other aerodynamic reduction devices. Our evaluation uses drag improvements corresponding to a reduction in the coefficient of aerodynamic drag, $C_{d}$, from a value of 0.62 to 0.52 . In addition to the individual technology evaluations, the emissions benefits corresponding to combinations of these technologies are evaluated by running MOVES with the various combinations of the modified parameter values.

Table 18 shows the MOVES predictions for the expected emissions reductions associated with these advanced efficiency technologies. It should be noted that the emissions analysis does not lend itself to an evaluation of the benefits of regenerative braking, so this is not included as it was for the analysis in previous sections of the report using the tractive energy analysis. The results indicate that emissions of $\mathrm{CO}$ and $\mathrm{HC}$ are rather insensitive to the variations evaluated. The change in emissions for these criteria emissions are less than $1 \%$ for all of the variations evaluated. For NOx emissions, the rolling resistance reduction and the aerodynamic drag reduction cases are similar, with each modification yielding a NOx emission reduction that is slightly over $4 \%$. The mass reduction considered, however, is predicted to be less effective, with a $1.4 \%$ reduction predicted. It is interesting to observe that for all of the technology combination cases considered, the combined NOx emission reduction is greater than the sum of the reductions provided by each individual technology case. The trends for the PM emission and fuel energy consumption results (which is also proportional to $\mathrm{CO}_{2}$ emissions) are similar to the NOx result, although the magnitudes of the predicted reductions differ somewhat. The MOVES model predicts a reduction in emissions for all of the technology implementation cases, except for the case of the HC emissions, which increase slightly while the other emissions are decreased. The $\mathrm{CO}$ emission for the reduced mass case is 
also slightly higher than the baseline case, but the level of the increase is only $0.7 \%$. The predictions do show that relatively significant emissions reductions can be achieved with the implementation of these technologies. By implementing all three of the technologies together, the model predicts that NOx, PM and $\mathrm{CO}_{2}$ emissions can be reduced by $11.0 \%, 8.3 \%$ and $12.4 \%$, respectively. 
Table 18: Emissions reductions associated with different advanced efficiency technologies as predicted by MOVES using the synthetic drive cycle representing the HTDC driving data.

\begin{tabular}{|c|c|c|c|}
\hline Metric & Description & $\begin{array}{c}\text { MOVES } \\
\text { result }\end{array}$ & $\begin{array}{l}\text { \% Difference } \\
\text { relative to } \\
\text { baseline case }\end{array}$ \\
\hline \multirow{6}{*}{$\begin{array}{c}\mathrm{NO}_{\mathrm{x}} \\
(\mathrm{g} / \mathrm{mi})\end{array}$} & Baseline vehicle configuration $^{*}$ & 7.721 & \\
\hline & Vehicle light-weighting (calculated assuming a 2,000 kg mass reduction) & 7.613 & $-1.4 \%$ \\
\hline & Low rolling resistance tires $\left(C_{R R}=0.0055\right)$ & 7.399 & $-4.2 \%$ \\
\hline & Aerodynamic drag reduction $\left(C_{d}=0.52\right)$ & 7.403 & $-4.1 \%$ \\
\hline & Low rolling resistance and aero drag reduction & 6.994 & $-9.4 \%$ \\
\hline & Change in mass $(2,000 \mathrm{~kg}$ reduction $) \& C_{R R}=0.0055 \& C_{d}=0.52$ & 6.875 & $-11.0 \%$ \\
\hline \multirow{6}{*}{$\begin{array}{c}\mathrm{CO} \\
(\mathrm{g} / \mathrm{mi})\end{array}$} & Baseline vehicle configuration & 1.512 & \\
\hline & Vehicle light-weighting (calculated assuming a 2,000 kg mass reduction) & 1.517 & $0.3 \%$ \\
\hline & Low rolling resistance tires $\left(C_{R R}=0.0055\right)$ & 1.508 & $-0.3 \%$ \\
\hline & Aerodynamic drag reduction $\left(C_{d}=0.52\right)$ & 1.509 & $-0.2 \%$ \\
\hline & Low rolling resistance and aero drag reduction & 1.498 & $-0.9 \%$ \\
\hline & Change in mass $(2,000 \mathrm{~kg}$ reduction $) \& C_{R R}=0.0055 \& C_{d}=0.52$ & 1.500 & $-0.8 \%$ \\
\hline \multirow{6}{*}{$\begin{array}{c}\mathrm{HC} \\
(\mathrm{g} / \mathrm{mi})\end{array}$} & Baseline vehicle configuration $^{*}$ & 0.345 & \\
\hline & Vehicle light-weighting (calculated assuming a 2,000 kg mass reduction) & 0.345 & $0.0 \%$ \\
\hline & Low rolling resistance tires $\left(C_{R R}=0.0055\right)$ & 0.346 & $0.3 \%$ \\
\hline & Aerodynamic drag reduction $\left(C_{d}=0.52\right)$ & 0.346 & $0.3 \%$ \\
\hline & Low rolling resistance and aero drag reduction & 0.347 & $0.6 \%$ \\
\hline & Change in mass $(2,000 \mathrm{~kg}$ reduction $) \& C_{R R}=0.0055 \& C_{d}=0.52$ & 0.348 & $0.9 \%$ \\
\hline \multirow{6}{*}{$\begin{array}{c}\mathrm{PM}_{2.5} \\
(\mathrm{OC}+\mathrm{EC}) \\
(\mathrm{g} / \mathrm{mi})\end{array}$} & Baseline vehicle configuration & 0.373 & \\
\hline & Vehicle light-weighting (calculated assuming a 2,000 kg mass reduction) & 0.366 & $-1.9 \%$ \\
\hline & Low rolling resistance tires $\left(C_{R R}=0.0055\right)$ & 0.361 & $-3.2 \%$ \\
\hline & Aerodynamic drag reduction $\left(C_{d}=0.52\right)$ & 0.362 & $-2.9 \%$ \\
\hline & Low rolling resistance and aero drag reduction & 0.348 & $-6.7 \%$ \\
\hline & Change in mass $\left(2,000 \mathrm{~kg}\right.$ reduction) $\& C_{R R}=0.0055 \& C_{d}=0.52$ & 0.342 & $-8.3 \%$ \\
\hline \multirow{6}{*}{$\begin{array}{c}\text { Total } \\
\text { energy } \\
\text { consumed } \\
(\mathrm{kJ} / \mathrm{mi})\end{array}$} & Baseline vehicle configuration & 20,574 & \\
\hline & Vehicle light-weighting (calculated assuming a 2,000 kg mass reduction) & 20,158 & $-2.0 \%$ \\
\hline & Low rolling resistance tires $\left(C_{R R}=0.0055\right)$ & 19,594 & $-4.8 \%$ \\
\hline & Aerodynamic drag reduction $\left(C_{d}=0.52\right)$ & 19,615 & $-4.7 \%$ \\
\hline & Low rolling resistance and aero drag reduction & 18,406 & $-10.5 \%$ \\
\hline & Change in mass $(2,000 \mathrm{~kg}$ reduction $) \& C_{R R}=0.0055 \& C_{d}=0.52$ & 18,019 & $-12.4 \%$ \\
\hline
\end{tabular}

Notes:

Emissions are based on mean base rate (w/out temperature \& humidity adjustments);

* Parameters for the baseline vehicle configuration: $m=24,187 \mathrm{~kg} ; C_{R R}=0.007 ; C_{d}=0.62$; frontal area $6.5 \mathrm{~m}^{2}$. 


\subsubsection{Comparison of results using an alternative drive cycle with those from the synthetic cycle}

In addition to presenting the results of the MOVES analysis based on the overall usage synthetic cycle, we also provide a comparison of these results to those corresponding to a different type of drive cycle. One approach the could be taken to select an appropriate drive cycle is to compare the characteristics of a large set of data and find a single measured drive cycle for which the characteristics are similar to the larger set, without doing the detailed matching of velocity and acceleration distributions that was done for the synthetic drive cycle. As a means to compare results for a measured drive cycle that is somewhat similar to those obtained using the overall usage synthetic drive cycle, a full day's measurement whose bivariate speed-acceleration histogram was visually similar to that of the complete HTDC data set was selected using the drive cycle tools (see section 4 for more details). This substitute measured drive cycle was evaluated with the MOVES model to determine how accurately the results compare with the synthetic cycle results.

Results of the comparison between the substitute measured drive cycle and the synthetic drive cycle are shown in Table 19, using the same format as in Table 17 except that the synthetic cycle, which is most representative of the complete set of driving data, is used as the reference case here. We observe that the absolute level of predicted emissions is certainly not as accurate as was the case in Table 17, but we wish to evaluate whether the predicted reductions (on a relative percentage basis) in the emissions can still be well estimated using the substitute measured drive cycle. In the present comparison, we see that the greatest difference between the predicted emissions reductions for the two drive cycles (column 6) is $3.4 \%$, where it was only $1.5 \%$ for the previous comparison. While the general trends for the MOVES-predicted emissions reductions are still correct using the substitute cycle, the magnitudes of the reductions are not as consistent with the synthetic cycle as one would like and relatively large errors in the magnitude of emissions reductions occur with the substitute drive cycle. For example, the model predicts a $9.4 \%$ reduction in NOx emission relative to the baseline configuration for the combination of reduced rolling resistance and reduced aerodynamic drag when using the synthetic drive cycle, but for the same case using the substitute measured drive cycle, the predicted NOx reduction is only $6.2 \%$. The $3.2 \%$ difference here represents about $1 / 3$ of the predicted benefit. This magnitude of error resulting from the use of the less representative drive cycle occurs in several of the comparisons in Table 19. Based on these results, it appears that using a drive cycle selected based on a general agreement with the application considered is not unreasonable for the prediction of general trends in emissions reductions, but errors in the magnitude of the predicted savings are expected to be greater using this approach than when a highly representative cycle can be used in the MOVES analysis. 
Table 19: Comparison of MOVES-predicted emissions using the synthetic drive cycle with those calculated using a substitute measured drive cycle.

\begin{tabular}{|c|c|c|c|c|c|c|}
\hline Metric & Description & $\begin{array}{l}\text { Synthetic } \\
\text { drive cycle }\end{array}$ & $\begin{array}{c}\% \\
\text { Difference } \\
\text { (synthetic) } \\
\text { [(chg-def)/def] }\end{array}$ & $\begin{array}{l}\text { Substitute } \\
\text { measured } \\
\text { drive cycle }\end{array}$ & $\begin{array}{c}\% \\
\text { Difference } \\
\text { (substitute) } \\
\text { [(chg-def)/def] }\end{array}$ & $\begin{array}{c}\text { Difference } \\
\text { between } \\
\text { \%subst \& } \\
\text { \%synthetic } \\
\text { [syn-full] }\end{array}$ \\
\hline \multirow{6}{*}{$\begin{array}{c}\mathrm{NO}_{x} \\
(\mathrm{~g} / \mathrm{mi})\end{array}$} & Default parameters (baseline vehicle configuration) & 7.721 & & 8.313 & & \\
\hline & Change in mass $(2,000 \mathrm{~kg}$ reduction $)$ & 7.613 & $-1.4 \%$ & 8.171 & $-1.7 \%$ & $0.3 \%$ \\
\hline & Change in $C_{R R}=0.0055$ & 7.399 & $-4.2 \%$ & 8.033 & $-3.4 \%$ & $-0.8 \%$ \\
\hline & Change in $C_{d}=0.52$ & 7.403 & $-4.1 \%$ & 8.058 & $-3.1 \%$ & $-1.0 \%$ \\
\hline & Change in $C_{R R}=0.0055$ \& $C_{d}=0.52$ & 6.994 & $-9.4 \%$ & 7.798 & $-6.2 \%$ & $-3.2 \%$ \\
\hline & Change in mass $\left(2,000 \mathrm{~kg}\right.$ reduction) \& $C_{R R}=0.0055 \& C_{d}=0.52$ & 6.875 & $-11.0 \%$ & 7.606 & $-8.5 \%$ & $-2.5 \%$ \\
\hline \multirow{6}{*}{$\begin{array}{c}\mathrm{CO} \\
(\mathrm{g} / \mathrm{mi})\end{array}$} & Default parameters (baseline vehicle configuration) & 1.512 & & 1.512 & & \\
\hline & Change in mass $(2,000 \mathrm{~kg}$ reduction $)$ & 1.517 & $0.3 \%$ & 1.517 & $0.3 \%$ & $0.0 \%$ \\
\hline & Change in $C_{R R}=0.0055$ & 1.508 & $-0.3 \%$ & 1.510 & $-0.1 \%$ & $-0.2 \%$ \\
\hline & Change in $C_{d}=0.52$ & 1.509 & $-0.2 \%$ & 1.511 & $-0.1 \%$ & $-0.1 \%$ \\
\hline & Change in $C_{R R}=0.0055 \& C_{d}=0.52$ & 1.498 & $-0.9 \%$ & 1.508 & $-0.3 \%$ & $-0.6 \%$ \\
\hline & Change in mass $(2,000 \mathrm{~kg}$ reduction $) \& C_{R R}=0.0055 \& C_{d}=0.52$ & 1.500 & $-0.8 \%$ & 1.512 & $0.0 \%$ & $-0.8 \%$ \\
\hline \multirow{6}{*}{$\begin{array}{c}\mathrm{HC} \\
(\mathrm{g} / \mathrm{mi})\end{array}$} & Default parameters (baseline vehicle configuration) & 0.345 & & 0.345 & & \\
\hline & Change in mass $(2,000 \mathrm{~kg}$ reduction $)$ & 0.345 & $0.0 \%$ & 0.345 & $0.0 \%$ & $0.0 \%$ \\
\hline & Change in $C_{R R}=0.0055$ & 0.346 & $0.3 \%$ & 0.345 & $0.0 \%$ & $0.3 \%$ \\
\hline & Change in $C_{d}=0.52$ & 0.346 & $0.3 \%$ & 0.345 & $0.0 \%$ & $0.3 \%$ \\
\hline & Change in $C_{R R}=0.0055 \& C_{d}=0.52$ & 0.347 & $0.6 \%$ & 0.346 & $0.3 \%$ & $0.3 \%$ \\
\hline & Change in mass $\left(2,000 \mathrm{~kg}\right.$ reduction) \& $C_{R R}=0.0055 \& C_{d}=0.52$ & 0.348 & $0.9 \%$ & 0.346 & $0.3 \%$ & $0.6 \%$ \\
\hline \multirow{6}{*}{$\begin{array}{c}\mathrm{PM}_{2.5} \\
(\mathrm{OC}+\mathrm{EC}) \\
(\mathrm{g} / \mathrm{mi})\end{array}$} & Default parameters (baseline vehicle configuration) & 0.373 & & 0.399 & & \\
\hline & Change in mass $(2,000 \mathrm{~kg}$ reduction) & 0.366 & $-1.9 \%$ & 0.392 & $-1.8 \%$ & $-0.1 \%$ \\
\hline & Change in $C_{R R}=0.0055$ & 0.361 & $-3.2 \%$ & 0.389 & $-2.5 \%$ & $-0.7 \%$ \\
\hline & Change in $C_{d}=0.52$ & 0.362 & $-2.9 \%$ & 0.390 & $-2.3 \%$ & $-0.6 \%$ \\
\hline & Change in $C_{R R}=0.0055 \& C_{d}=0.52$ & 0.348 & $-6.7 \%$ & 0.381 & $-4.5 \%$ & $-2.2 \%$ \\
\hline & Change in mass $(2,000 \mathrm{~kg}$ reduction $) \& C_{R R}=0.0055 \& C_{d}=0.52$ & 0.342 & $-8.3 \%$ & 0.372 & $-6.8 \%$ & $-1.5 \%$ \\
\hline \multirow{6}{*}{$\begin{array}{c}\text { Total } \\
\text { energy } \\
\text { consumed } \\
\text { (kJ/mi) }\end{array}$} & Default parameters (baseline vehicle configuration) & 20574 & & 22,291 & & \\
\hline & Change in mass $(2,000 \mathrm{~kg}$ reduction) & 20158 & $-2.0 \%$ & 21,773 & $-2.3 \%$ & $0.3 \%$ \\
\hline & Change in $C_{R R}=0.0055$ & 19594 & $-4.8 \%$ & 21,426 & $-3.9 \%$ & $-0.9 \%$ \\
\hline & Change in $C_{d}=0.52$ & 19615 & $-4.7 \%$ & 21,502 & $-3.5 \%$ & $-1.2 \%$ \\
\hline & Change in $C_{R R}=0.0055 \& C_{d}=0.52$ & 18406 & $-10.5 \%$ & 20,714 & $-7.1 \%$ & $-3.4 \%$ \\
\hline & Change in mass $(2,000 \mathrm{~kg}$ reduction $) \& C_{R R}=0.0055 \& C_{d}=0.52$ & 18019 & $-12.4 \%$ & 20,101 & $-9.8 \%$ & $-2.6 \%$ \\
\hline
\end{tabular}

Notes: Emissions are based on mean base rate (w/out temperature \& humidity adjustments); default parameters: $m=24,187 \mathrm{~kg} ; \mathrm{C}_{R R}=0.007 ; \mathrm{C}_{\mathrm{d}}=0.62 ;$ frontal area $6.5 \mathrm{~m}{ }^{2}$. chg = changed parameter case; def = default parameter case; syn = synthetic cycle; subst = substitute measured drive cycle 


\subsubsection{Comparison of emissions estimates for synthetic and real drive cycles versus the default MOVES drive cycles}

As a final comparison, default drive cycles that are included in the MOVES model were evaluated to determine how well emissions can be estimated without the availability of specific drive cycle data representing the actual usage of a fleet or trucking application. For this evaluation, we also compare the operating mode distributions, as they are defined in the MOVES model, among all three drive cycles compared in sections 6.3.4 and 6.3.4: the overall usage synthetic drive cycle, the substitute measured drive cycle and the MOVES default drive cycle.

In MOVES, a basic emissions evaluation can be performed by selecting an average driving speed for the application considered. MOVES will identify relevant default drive cycles that are included in the software and weight the results between two of these cycles so that the corresponding weighted average speed matches that input into the model. To run MOVES using the model's default drive cycles, both an average vehicle speed and average road grade (in percent grade) are required as inputs. A difficulty in using this average speed method is in defining the average vehicle speed. The usage for the fleet evaluated contained nearly $50 \%$ idling, so it is unreasonable to use the entire drive cycle to calculate average vehicle speed since the vehicle spent almost half of its time at zero speed. In addition, road grade varied between positive and negative values, and the average road grade is typically closer to zero when the positive and negative grades are averaged together. To work around these problems, average vehicle speed was determined by removing all idling greater than 1 minute in duration from the synthetic drive cycle and the average grade was determined separately for positive and negative values. Using this method, total idling, based on the MOVES definition, was reduced to $7 \%$ for the synthetic cycle. Separate average positive and average negative road grades were then determined using the condensed vehicle speed data. With the long idling data removed from the synthetic drive cycle, the modified average speed was $88.8 \mathrm{~km} / \mathrm{h}(55.2 \mathrm{mph})$, while the average positive road grade was $0.9 \%$, and average negative road grade was $-1.0 \%$. Using these results, MOVES was run separately for the positive and negative road grade, each at the average speed calculated for the synthetic drive cycle, and the results from the two MOVES runs were averaged together and compared to the synthetic drive cycle.

The results of the MOVES default drive cycle evaluation following this method are shown in Table 20 and are compared with the results using the synthetic drive cycle. The synthetic cycle results are the same as were shown in Table 18, and the format of the table is the same as was used in the previous cycle-tocycle comparisons. The results for the predicted emissions reductions corresponding to the efficiency technology simulations are somewhat mixed. As in the case of the substitute drive cycle comparison (Table 19), the predicted trends for emissions reductions are generally consistent between the MOVES default drive cycle and the synthetic drive cycle. However, the magnitudes of the predicted emissions reductions again show some discrepancies that are noteworthy. The reduced mass parameter case showed only a moderate emissions reduction ( $\leq 2 \%$ for each of the metrics evaluated) when using the synthetic drive cycle, but the predicted emissions reductions using the default MOVES drive cycle is considerably higher for some cases. The predicted NOx emission reduction associated with a $2000 \mathrm{~kg}$ mass reduction using the MOVES default drive cycle is 3.8\%, which is more than 2.5 times the value of $1.4 \%$ predicted with the synthetic drive cycle. Also, similar to the evaluation with the substitute drive 
cycle, the predicted NOx and total energy consumption reductions due to a combination of rolling resistance and aerodynamic drag reductions was about $1 / 3$ less than the estimate using the synthetic drive cycle. The differences between results using different drive cycles are significant relative to the benefits achieved, which highlights the need to use drive cycles that are as representative of the actual application as possible. These results using the MOVES default drive cycle suggest that the cycles could be improved for freeway-dominant usage cases such as the HTDC fleet usage.

When the average speed is input into MOVES to use its default drive cycles for estimating emissions, it selects two cycles, with lower and higher average speeds, and weights them to achieve the same average speed as input. The operating mode distribution is then generated based on this weighting of the two default drive cycles. For the case analyzed above, the two drive cycles selected (out of the available 12 default drive cycles for HD trucks [See Table 15]) had average speeds of $87.2 \mathrm{~km} / \mathrm{h}(54.2$ $\mathrm{mph}$ ) and $95.6 \mathrm{~km} / \mathrm{h}(59.4 \mathrm{mph})$, as compared to the average speed of $88.8 \mathrm{~km} / \mathrm{h}(55.2 \mathrm{mph})$ from the synthetic drive cycles. The speed-time plots for these two default cycles are shown in Fig. 45.

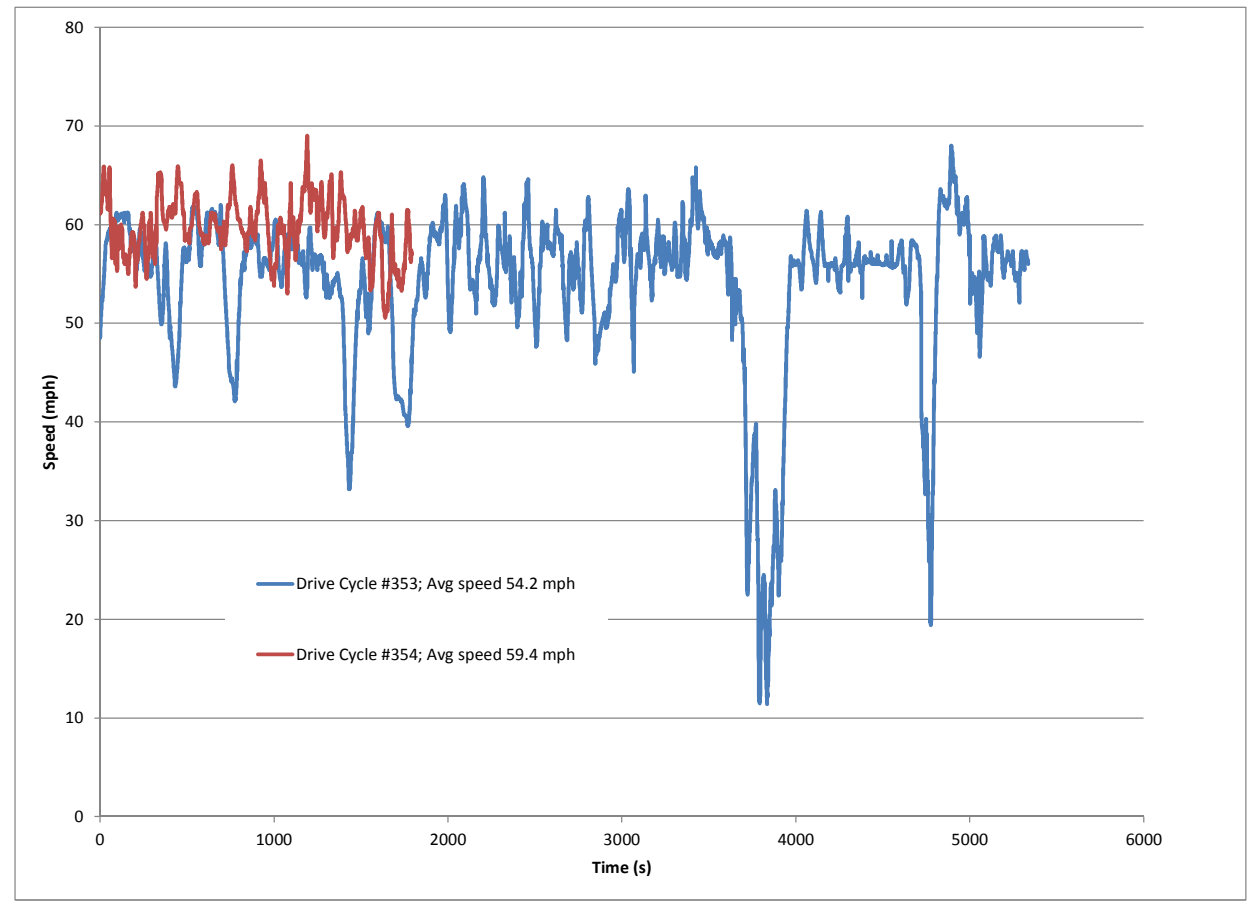

Figure 45 MOVES default cycles for average speeds $87.2 \mathrm{~km} / \mathrm{h}(54.2 \mathrm{mph})$ and $95.6 \mathrm{~km} / \mathrm{h}(59.4 \mathrm{mph})$ 
Table 20: Comparison of the overall usage synthetic drive cycle and the MOVES default drive cycle based on the average speed of the synthetic cycle.

\begin{tabular}{|c|c|c|c|c|c|c|}
\hline Metric & Description & $\begin{array}{l}\text { Synthetic } \\
\text { drive cycle }\end{array}$ & $\begin{array}{c}\text { \% Difference } \\
\text { (synthetic) } \\
\text { [(chg-def)/def] }\end{array}$ & $\begin{array}{c}\text { MOVES } \\
\text { default } \\
\text { drive cycle }\end{array}$ & $\begin{array}{c}\text { \% Difference } \\
\text { (MOVES default) } \\
\text { [(chg-def)/def] }\end{array}$ & $\begin{array}{c}\text { Difference } \\
\text { between \%syn } \\
\text { \& \%MOVES } \\
\text { [syn- MOVES] }\end{array}$ \\
\hline \multirow{6}{*}{$\begin{array}{c}\mathrm{NO}_{\mathrm{x}} \\
(\mathrm{g} / \mathrm{mi})\end{array}$} & "Default parameters (baseline vehicle configuration) & 7.721 & & 7.252 & & \\
\hline & Change in mass $(2,000 \mathrm{~kg}$ reduction $)$ & 7.613 & $-1.4 \%$ & 6.978 & $-3.8 \%$ & $2.4 \%$ \\
\hline & Change in $C_{R R}=0.0055$ & 7.399 & $-4.2 \%$ & 7.000 & $-3.5 \%$ & $-0.7 \%$ \\
\hline & Change in $C_{d}=0.52$ & 7.403 & $-4.1 \%$ & 7.070 & $-2.5 \%$ & $-1.6 \%$ \\
\hline & Change in $C_{R R}=0.0055 \& C_{d}=0.52$ & 6.994 & $-9.4 \%$ & 6.817 & $-6.0 \%$ & $-3.4 \%$ \\
\hline & Change in mass $(2,000 \mathrm{~kg}$ reduction $) \& C_{R R}=0.0055 \& C_{d}=0.52$ & 6.875 & $-11.0 \%$ & 6.524 & $-10.0 \%$ & $-1.0 \%$ \\
\hline \multirow{6}{*}{$\begin{array}{c}\mathrm{CO} \\
(\mathrm{g} / \mathrm{mi})\end{array}$} & Default parameters (baseline vehicle configuration) & 1.512 & & 1.364 & & \\
\hline & Change in mass (2,000 $\mathrm{kg}$ reduction) & 1.517 & $0.3 \%$ & 1.363 & $-0.1 \%$ & $0.4 \%$ \\
\hline & Change in $C_{R R}=0.0055$ & 1.508 & $-0.3 \%$ & 1.359 & $-0.4 \%$ & $0.1 \%$ \\
\hline & Change in $C_{d}=0.52$ & 1.509 & $-0.2 \%$ & 1.360 & $-0.3 \%$ & $0.1 \%$ \\
\hline & Change in $C_{R R}=0.0055 \& C_{d}=0.52$ & 1.498 & $-0.9 \%$ & 1.354 & $-0.7 \%$ & $-0.2 \%$ \\
\hline & Change in mass $\left(2,000 \mathrm{~kg}\right.$ reduction) $\& C_{R R}=0.0055 \& C_{d}=0.52$ & 1.500 & $-0.8 \%$ & 1.352 & $-0.9 \%$ & $0.1 \%$ \\
\hline \multirow{6}{*}{$\begin{array}{c}\mathrm{HC} \\
(\mathrm{g} / \mathrm{mi})\end{array}$} & Default parameters (baseline vehicle configuration) & 0.345 & & 0.284 & & \\
\hline & Change in mass $(2,000 \mathrm{~kg}$ reduction $)$ & 0.345 & $0.0 \%$ & 0.284 & $0.0 \%$ & $0.0 \%$ \\
\hline & Change in $C_{R R}=0.0055$ & 0.346 & $0.3 \%$ & 0.284 & $0.0 \%$ & $0.3 \%$ \\
\hline & Change in $C_{d}=0.52$ & 0.346 & $0.3 \%$ & 0.284 & $0.0 \%$ & $0.3 \%$ \\
\hline & Change in $C_{R R}=0.0055 \& C_{d}=0.52$ & 0.347 & $0.6 \%$ & 0.285 & $0.4 \%$ & $0.2 \%$ \\
\hline & Change in mass $\left(2,000 \mathrm{~kg}\right.$ reduction) $\& C_{R R}=0.0055 \& C_{d}=0.52$ & 0.348 & $0.9 \%$ & 0.285 & $0.4 \%$ & $0.5 \%$ \\
\hline \multirow{6}{*}{$\begin{array}{c}\mathrm{PM}_{2.5} \\
(\mathrm{OC}+\mathrm{EC}) \\
(\mathrm{g} / \mathrm{mi})\end{array}$} & Default parameters (baseline vehicle configuration) & 0.373 & & 0.338 & & \\
\hline & Change in mass $(2,000 \mathrm{~kg}$ reduction $)$ & 0.366 & $-1.9 \%$ & 0.326 & $-3.6 \%$ & $1.7 \%$ \\
\hline & Change in $C_{R R}=0.0055$ & 0.361 & $-3.2 \%$ & 0.329 & $-2.7 \%$ & $-0.5 \%$ \\
\hline & Change in $C_{d}=0.52$ & 0.362 & $-2.9 \%$ & 0.332 & $-1.8 \%$ & $-1.1 \%$ \\
\hline & Change in $C_{R R}=0.0055 \& C_{d}=0.52$ & 0.348 & $-6.7 \%$ & 0.322 & $-4.7 \%$ & $-2.0 \%$ \\
\hline & Change in mass $\left(2,000 \mathrm{~kg}\right.$ reduction) $\& C_{R R}=0.0055 \& C_{d}=0.52$ & 0.342 & $-8.3 \%$ & 0.310 & $-8.3 \%$ & $0.0 \%$ \\
\hline \multirow{6}{*}{$\begin{array}{c}\text { Total } \\
\text { energy } \\
\text { consumed } \\
(\mathrm{kJ} / \mathrm{mi})\end{array}$} & Default parameters (baseline vehicle configuration) & 20574 & & 21178 & & \\
\hline & Change in mass $(2,000 \mathrm{~kg}$ reduction $)$ & 20158 & $-2.0 \%$ & 20249 & $-4.4 \%$ & $2.4 \%$ \\
\hline & Change in $C_{R R}=0.0055$ & 19594 & $-4.8 \%$ & 20378 & $-3.8 \%$ & $-1.0 \%$ \\
\hline & Change in $C_{d}=0.52$ & 19615 & $-4.7 \%$ & 20605 & $-2.7 \%$ & $-2.0 \%$ \\
\hline & Change in $C_{R R}=0.0055 \& C_{d}=0.52$ & 18406 & $-10.5 \%$ & 19808 & $-6.5 \%$ & $-4.0 \%$ \\
\hline & Change in mass $\left(2,000 \mathrm{~kg}\right.$ reduction) $\& C_{R R}=0.0055 \& C_{d}=0.52$ & 18019 & $-12.4 \%$ & 18844 & $-11.0 \%$ & $-1.4 \%$ \\
\hline
\end{tabular}

Notes: Emissions are based on mean base rate (w/out temperature \& humidity adjustments); default parameters: $\mathrm{m}=24,187 \mathrm{~kg} ; \mathrm{C}_{\mathrm{RR}}=0.007 ; \mathrm{C}_{\mathrm{d}}=0.62 ;$ frontal area $6.5 \mathrm{~m}{ }^{2}$. chg = changed parameter case; def = default parameter case; syn = synthetic cycle; MOVES = MOVES default drive cycle 
It can be seen that these cycles only represent the on-freeway portion of usage since they do not contain any low speed driving or engine idle operation. As a consequence, the driving segments included in the default MOVES drive cycle lacks a range of the operating conditions experienced in the real drive cycle, even if the overall contribution from the low speed conditions is relatively small. Figure 46 shows a comparison of the operating mode distributions for the simulations considered above, using (1) the MOVES default cycle based on the average speed of the synthetic drive cycle, (2) the synthetic drive cycle, and (3) the substitute measured drive cycle. (Note: Table 14 contains a list of the vehicle speed and STP conditions for the operating mode bins.) As revealed in the figure, the operating mode distributions for these cycles include similar proportions of the high-speed conditions; however, the relative magnitudes of the distributions are different for the MOVES default cycle case since it does not include any of the low speed or idle operation conditions. More than twice as much time was spent in bin 33 for the MOVES average speed cycles relative to the full and synthetic cycles, and similarly but at a smaller scale for bin 35, as well. Both of these bins belong to the speed range of $80 \mathrm{~km} / \mathrm{h}(50 \mathrm{mph})$ and above. It is clear that this difference in the operating mode fraction is responsible for the differences in predicted emissions for the analysis case in which the MOVES default cycles were used to correspond to the average speed of the synthetic drive cycle.

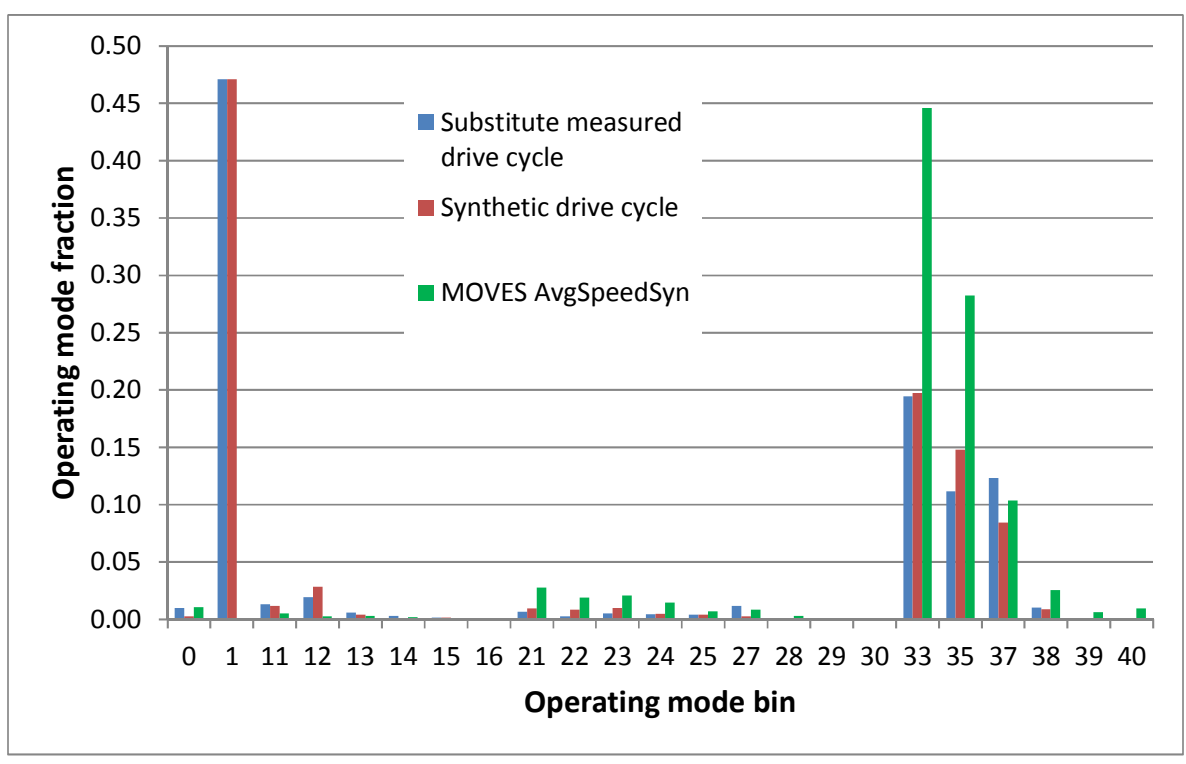

Figure 46 Operating mode bin distribution for the original, synthetic, and MOVES average speed cycles

\subsection{Summary and Conclusions of the MOVES analysis}

Four areas were investigated with the MOVES modeling activity in this project: (1) second-by-second emission estimates from MOVES were compared to emission measurements conducted during a dynamometer drive cycle; (2) emissions estimates using a real world drive cycle from a HD diesel truck were compared with emissions estimates from a representative synthetic drive cycle in order to validate the synthetic drive cycle analysis methodology; (3) advanced efficiency technologies for class 8 long-haul 
trucks were assessed in terms of the emission reductions that can be achieved when implementing the technologies; and (4) emissions predictions based on default drive cycles in MOVES were compared to those using the synthetic drive cycle corresponding to the overall usage of the HTDC driving data. Primary findings from the MOVES analysis include the following:

- Emission estimates of $\mathrm{CO}$ and $\mathrm{HC}$ using the MOVES model did not match measured emissions as well as those for $\mathrm{NO}_{\mathrm{x}}$ and $\mathrm{PM}_{2.5}$, and the predictions of $\mathrm{CO}$ and $\mathrm{HC}$ emissions reductions also showed less sensitivity to vehicle configuration changes than did the $\mathrm{NO}_{\mathrm{x}}$ and $\mathrm{PM}$ emissions.

- MOVES emission estimates using a synthetic drive cycle were confirmed to agree very closely with those obtained from an original drive cycle that the synthetic cycle represents, and the predicted emissions reductions corresponding to vehicle configuration changes matched to within $1.5 \%$ for all of the cases evaluated.

- MOVES can be used to quantify the emissions reductions achievable with various fuel efficiency technologies, such as those associated with rolling resistance, aerodynamic drag, and weight reduction, by adjusting parameters contained in the input database. Individual technologies and combinations of these technologies were assessed using MOVES for the vehicle usage measured during ORNL's previous Heavy Truck Duty Cycle (HTDC) project.

The comparison of second-by-second emission estimates between the dynamometer test from a typical freeway-based drive cycle and those predicted by MOVES for the same cycle showed general agreement for $\mathrm{NO}_{\mathrm{x}}$ emissions. However, $\mathrm{CO}$ and $\mathrm{HC}$ emissions did not show good agreement with the measured data. Nonetheless, the model compared favorably when aggregated emissions were compared to measured emissions even though the model was not designed for generating emissions for a single truck.

A synthetic drive cycle generated from measured driving data for a complete day of driving was evaluated for its ability to accurately represent the original drive cycle data. In particular, we compared MOVES estimated emission reductions that result when variations in vehicle parameters, corresponding to the implementation of advanced efficiency technologies, are made to the MOVES model. The validation study demonstrated that the results using the synthetic drive cycle compared very favorably with the MOVES predictions made with the original data on which the synthetic cycle was based. The results of the validation synthetic drive cycle matched the original cycle's MOVES predictions for emissions reductions to within $1.5 \%$ for all of the cases considered. This validation gives a high degree of confidence to the relevance of MOVES estimates made using a synthetic drive cycle.

The level of emission reductions for changes in vehicle parameters that are believed to be appropriate for real technology modifications including low rolling resistance tires, aerodynamic drag, and vehicle light-weighting were calculated using the MOVES model. These results, evaluated using a synthetic drive cycle developed to represent the overall usage of a class 8 tractor-trailer truckload delivery fleet, are representative of the emissions reductions that would be achieved overall in the fleet studied if the technologies are implemented for all vehicles in service. This was the key result for this analysis, and the emissions reductions estimated with MOVES for this evaluation are presented in Table 18. Since the MOVES emissions rates are typically used to evaluate emissions for the broader U.S. trucking fleet, we 
can conclude that the predicted emissions reductions are appropriate to all class 8 tractor-trailers if this fleet's usage is typical of most fleets operating in the same vehicle application. Further research is needed to determine if this usage is relevant to other class 8 trucking fleets.

\section{Recommendations for Future Research}

The results of the tractive energy analysis showed fuel savings as high as $10 \%$ from a regenerative braking system in the class 8 freight delivery application evaluated. Although fuel savings of $20-30 \%$ are realized with medium duty hybrid trucks using regenerative braking in operations for which considerable stop and go activity is present, the conventional belief is that hybridization will not generate significant savings for class 8 tractor-trailers. The drive cycle for the fleet studied showed intermediate occurrences of low deceleration levels at speeds below $50 \mathrm{mph}$, in addition to a relatively high occurrence of low to moderate decelerations at highway speeds. An understanding of how a class 8 hybrid system could be implemented to recover the braking energy for such a drive profile is needed to determine if the fuel savings predicted using the tractive energy model could be achieved in practice. Detailed modeling studies of class 8 tractor trailers using a realistic regenerative braking configuration and employing a drive cycle that is highly representative of class 8 freight operations (such as the synthetic drive cycle developed for this project) would help determine if the assumptions used in this study are reasonable. A characterization of heavy duty truck regenerative braking systems, including their power and energy storage limitations, would also help define a more realistic set of assumptions to use when conducting a tractive energy analysis.

There is a need to confirm that the overall usage from the fleet evaluated in this study is typical of class 8 freight transport operations. Detailed drive cycle evaluations in other class 8 shipping fleets would be helpful to verify that the fuel efficiency gains predicted in this study are representative of the broader U.S. fleet. This application consumes more fuel than any other in U.S. trucking so it is important to understand where the greatest opportunities for fuel savings exist. On the other hand, there are many other trucking applications for which the typical usage is very poorly understood. Studies to quantify the usage across the full spectrum of U.S. trucking operations would allow much better decisions to be made regarding appropriate fuel efficiency technologies to implement in the other applications.

The results presented in section 6 of this report showed some weaknesses in the MOVES model in terms of how the usage is selected using the built-in drive cycles. MOVES currently will select drive cycles from a finite set of basis drive cycle segments, and it weights these to achieve the same average speed as what the user inputs when running the model. Alternative approaches or the development of additional basis drive cycles should be considered so that real usage cases can be better represented when using the average speed method for MOVES simulations. Different usage scenarios should be studied in MOVES to determine how to best represent a particular usage using the average speed and other characteristics of a fleet's operations. Even for freeway-dominant drive cycles, there needs to be some weighting of non-freeway operations, and the evaluation of several different fleets and applications could be pursued to generate a more accurate methodology for default drive cycle generation. 


\section{References:}

1. Capps, G., O. Franzese, B. Knee, M.B. Lascurain, and P. Otaduy, Class-8 Heavy Truck Duty Cycle Project Final Report. Report No. ORNL/TM-2008/122. Oak Ridge National Laboratory, 2008.

2. LaClair, T., "Application of a Tractive Energy Analysis to Quantify the Benefits of Advanced Efficiency Technologies for Medium- and Heavy-Duty Trucks Using Characteristic Drive Cycle Data," SAE Int. J. Commer. Veh. 5(1):141-163, 2012, doi:10.4271/2012-01-0361.

3. M.D. Surcel, "Energotest 2007: Fuel Consumption Test for Evaluating Freight Wing Trailer Side Skirts," Internal Report IR-2007-11-28D, FP Innovations. http://www.freightwing.com/docs/IR2007-11-28D-MSL-Energotest2007-FreightWing.pdf, Accessed November 1, 2012.

4. Clark, N.N., Gautum, M., Bata, R.M., Loth, J., Palmer, G.M., Wang, W.G., and Lyons, D.W., "Design and Operation of a New Transportable Laboratory for Emissions Testing of Heavy Duty Trucks and Buses," International Journal of Vehicle Design (Heavy Vehicle Systems), Vol. 2, Nos. 3/4, 1995, pp. 285-299.

5. DeLorme GPS Community Forum, "Why is DeLorme Vertically or Horizontally inaccurate?" http://forum.delorme.com/viewtopic.php?t=11777, accessed 10/15/2012.

6. American Association of State Highway and Transportation Officials (AASHTO), A Policy on Geometric Design of Highways and Streets, 6th Edition, AASHTO, 2011.

7. Argonne National Laboratory, Autonomie, http://www.autonomie.net, accessed October 15, 2012.

8. Barth, M.; An, F.; Norbeck, J.; Ross, M. "Modal emissions modeling: a physical approach," Transportation Research Record, 1520, pp. 81-88, 1996.

9. Kean, A.J.; Harley, R.A.; Kendal, G.R. "Effects of vehicle speed and engine load on motor vehicle emissions," Environmental Science and Technology, 37(17), pp. 3739-3746, 2003.

10. Zhang, K.; Frey, H.C. "Road grade estimation for on-road vehicle emissions modeling using LIDAR data," Journal of the Air and Waste Management Association, 56(6), pp. 777-788, 2006.

11. Jimenez-Palacios, J.L. Understanding and quantifying motor vehicle emissions with vehicle specific power and TILDAS remote sensing, Ph.D. Thesis, Massachusetts Institute of Technology, Cambridge, MA. 1999.

12. U.S. Environmental Protection Agency, MOVES web site, www.epa.gov/otaq/models/moves/index.htm, accessed November 1, 2012.

13. U.S. Environmental Protection Agency, Development of emission rates for heavy-duty vehicles in the Motor Vehicle Emissions Simulator MOVES2010 - Final Report, Assessment and Standards Division, Office of Transportation and Air Quality, EPA-420-B-12-049, August 2012.

14. U.S. Environmental Protection Agency, Diesel retrofits: Quantifying and using their benefits in SIPs and conformity - Guidance for State and Local Air and Transportation Agencies, Transportation and Regional Programs Division and Compliance and Innovative Strategies Division, Office of Transportation and Air Quality. EPA-420-B-06-005, June 2006.

15. U.S. Environmental Protection Agency, SmartWay SIP and transportation conformity guidance: Accounting for $\mathrm{NO}_{x}$ reductions from trailer aerodynamic kits and low rolling resistance tires Guidance for State and Local Air and Transportation Agencies, Transportation and Regional Programs Division, Office of Transportation and Air Quality. EPA-420-B-07-004, June 2007. 
16. U.S. Environmental Protection Agency, Development of emission rates for light-duty vehicles in the Motor Vehicle Emissions Simulator MOVES2009 - Draft Report. Assessment and Standards Division, Office of Transportation and Air Quality, EPA-420-P-09-002, August 2009. 


\section{APPENDIX - Table of the synthetic drive cycle representing the full usage of HTDC drive data}

\begin{tabular}{|c|c|c|}
\hline Time (s) & $\begin{array}{l}\text { Speed } \\
\text { (mph) }\end{array}$ & $\begin{array}{l}\text { Elevation } \\
(\mathrm{m})\end{array}$ \\
\hline 1 & 0.00 & 200.0 \\
\hline 2 & 0.00 & 200.0 \\
\hline 3 & 0.00 & 200.0 \\
\hline 4 & 0.00 & 200.0 \\
\hline 5 & 0.00 & 200.0 \\
\hline 6 & 0.00 & 200.0 \\
\hline 7 & 0.00 & 200.0 \\
\hline 8 & 0.00 & 200.0 \\
\hline 9 & 0.00 & 200.0 \\
\hline 10 & 0.00 & 200.0 \\
\hline 11 & 0.00 & 200.0 \\
\hline 12 & 0.00 & 200.0 \\
\hline 13 & 0.00 & 200.0 \\
\hline 14 & 1.43 & 200.0 \\
\hline 15 & 1.60 & 200.0 \\
\hline 16 & 1.67 & 200.0 \\
\hline 17 & 1.41 & 200.0 \\
\hline 18 & 1.38 & 200.0 \\
\hline 19 & 1.35 & 200.0 \\
\hline 20 & 1.43 & 200.0 \\
\hline 21 & 1.47 & 200.0 \\
\hline 22 & 2.29 & 200.0 \\
\hline 23 & 2.31 & 200.0 \\
\hline 24 & 2.30 & 200.0 \\
\hline 25 & 2.36 & 200.0 \\
\hline 26 & 2.38 & 200.0 \\
\hline 27 & 2.48 & 200.0 \\
\hline 28 & 3.75 & 200.0 \\
\hline 29 & 3.85 & 200.0 \\
\hline 30 & 3.80 & 200.0 \\
\hline 31 & 4.33 & 200.0 \\
\hline 32 & 4.41 & 200.0 \\
\hline 33 & 4.46 & 200.0 \\
\hline 34 & 4.37 & 200.0 \\
\hline 35 & 4.28 & 200.0 \\
\hline 36 & 4.20 & 200.0 \\
\hline 37 & 4.33 & 200.0 \\
\hline 38 & 4.35 & 200.0 \\
\hline 39 & 4.13 & 200.0 \\
\hline 40 & 5.59 & 200.0 \\
\hline 41 & 5.73 & 200.0 \\
\hline 42 & 5.78 & 200.0 \\
\hline 43 & 6.48 & 200.0 \\
\hline 44 & 7.07 & 200.0 \\
\hline 45 & 7.22 & 200.0 \\
\hline 46 & 7.20 & 200.0 \\
\hline 47 & 7.27 & 200.0 \\
\hline 48 & 7.31 & 200.0 \\
\hline 49 & 8.46 & 200.0 \\
\hline 50 & 8.63 & 200.0 \\
\hline 51 & 9.07 & 200.0 \\
\hline 52 & 9.03 & 200.0 \\
\hline
\end{tabular}

\begin{tabular}{|c|c|c|}
\hline Time (s) & $\begin{array}{l}\text { Speed } \\
\text { (mph) }\end{array}$ & $\begin{array}{l}\text { Elevation } \\
\text { (m) }\end{array}$ \\
\hline 53 & 8.95 & 200.0 \\
\hline 54 & 8.35 & 200.0 \\
\hline 55 & 8.12 & 200.0 \\
\hline 56 & 7.51 & 200.0 \\
\hline 57 & 7.35 & 200.0 \\
\hline 58 & 7.26 & 200.0 \\
\hline 59 & 6.94 & 200.0 \\
\hline 60 & 6.85 & 200.0 \\
\hline 61 & 6.81 & 200.0 \\
\hline 62 & 6.80 & 200.0 \\
\hline 63 & 5.43 & 200.0 \\
\hline 64 & 5.29 & 200.0 \\
\hline 65 & 5.22 & 200.0 \\
\hline 66 & 5.17 & 200.0 \\
\hline 67 & 5.14 & 200.0 \\
\hline 68 & 4.61 & 200.0 \\
\hline 69 & 4.44 & 200.0 \\
\hline 70 & 3.90 & 200.0 \\
\hline 71 & 3.73 & 200.0 \\
\hline 72 & 3.65 & 200.0 \\
\hline 73 & 3.69 & 200.0 \\
\hline 74 & 3.65 & 200.0 \\
\hline 75 & 2.96 & 200.0 \\
\hline 76 & 2.52 & 200.0 \\
\hline 77 & 2.37 & 200.0 \\
\hline 78 & 2.31 & 200.0 \\
\hline 79 & 2.24 & 200.0 \\
\hline 80 & 1.93 & 200.0 \\
\hline 81 & 1.83 & 200.0 \\
\hline 82 & 1.88 & 200.0 \\
\hline 83 & 1.83 & 200.0 \\
\hline 84 & 1.77 & 200.0 \\
\hline 85 & 0.96 & 200.0 \\
\hline 86 & 0.00 & 200.0 \\
\hline 87 & 0.00 & 200.0 \\
\hline 88 & 0.00 & 200.0 \\
\hline 89 & 0.00 & 200.0 \\
\hline 90 & 0.00 & 200.0 \\
\hline 91 & 0.00 & 200.0 \\
\hline 92 & 0.00 & 200.0 \\
\hline 93 & 0.00 & 200.0 \\
\hline 94 & 0.00 & 200.0 \\
\hline 95 & 0.00 & 200.0 \\
\hline 96 & 0.00 & 200.0 \\
\hline 97 & 0.00 & 200.0 \\
\hline 98 & 0.00 & 200.0 \\
\hline 99 & 0.00 & 200.0 \\
\hline 100 & 0.00 & 200.0 \\
\hline 101 & 0.00 & 200.0 \\
\hline 102 & 0.00 & 200.0 \\
\hline 103 & 0.00 & 200.0 \\
\hline 104 & 0.00 & 200.0 \\
\hline
\end{tabular}




\begin{tabular}{|c|c|c|}
\hline Time (s) & $\begin{array}{l}\text { Speed } \\
\text { (mph) }\end{array}$ & $\begin{array}{l}\text { Elevation } \\
(\mathrm{m})\end{array}$ \\
\hline 105 & 0.00 & 200.0 \\
\hline 106 & 0.00 & 200.0 \\
\hline 107 & 0.00 & 200.0 \\
\hline 108 & 0.00 & 200.0 \\
\hline 109 & 0.00 & 200.0 \\
\hline 110 & 0.00 & 200.0 \\
\hline 111 & 0.00 & 200.0 \\
\hline 112 & 0.00 & 200.0 \\
\hline 113 & 0.00 & 200.0 \\
\hline 114 & 0.00 & 200.0 \\
\hline 115 & 0.00 & 200.0 \\
\hline 116 & 0.00 & 200.0 \\
\hline 117 & 0.00 & 200.0 \\
\hline 118 & 0.00 & 200.0 \\
\hline 119 & 0.00 & 200.0 \\
\hline 120 & 0.00 & 200.0 \\
\hline 121 & 0.00 & 200.0 \\
\hline 122 & 0.00 & 200.0 \\
\hline 123 & 0.00 & 200.0 \\
\hline 124 & 0.00 & 200.0 \\
\hline 125 & 0.45 & 200.0 \\
\hline 126 & 1.37 & 200.0 \\
\hline 127 & 2.57 & 200.0 \\
\hline 128 & 2.86 & 200.0 \\
\hline 129 & 3.36 & 200.0 \\
\hline 130 & 3.43 & 200.0 \\
\hline 131 & 3.51 & 200.0 \\
\hline 132 & 4.84 & 200.0 \\
\hline 133 & 5.52 & 200.0 \\
\hline 134 & 5.57 & 200.0 \\
\hline 135 & 5.61 & 200.0 \\
\hline 136 & 6.10 & 200.0 \\
\hline 137 & 6.30 & 200.0 \\
\hline 138 & 6.35 & 200.0 \\
\hline 139 & 6.39 & 200.0 \\
\hline 140 & 6.49 & 200.0 \\
\hline 141 & 7.76 & 200.0 \\
\hline 142 & 8.38 & 200.0 \\
\hline 143 & 8.42 & 200.0 \\
\hline 144 & 8.66 & 200.0 \\
\hline 145 & 9.35 & 200.0 \\
\hline 146 & 9.57 & 200.0 \\
\hline 147 & 9.64 & 200.0 \\
\hline 148 & 10.70 & 200.0 \\
\hline 149 & 10.84 & 200.0 \\
\hline 150 & 10.79 & 200.0 \\
\hline 151 & 10.82 & 200.0 \\
\hline 152 & 11.74 & 200.0 \\
\hline 153 & 11.76 & 200.0 \\
\hline 154 & 11.68 & 200.0 \\
\hline 155 & 11.52 & 200.0 \\
\hline 156 & 11.93 & 200.0 \\
\hline 157 & 11.45 & 200.0 \\
\hline 158 & 12.34 & 200.0 \\
\hline 159 & 12.87 & 200.0 \\
\hline 160 & 12.88 & 200.0 \\
\hline
\end{tabular}

\begin{tabular}{|c|c|c|}
\hline Time (s) & $\begin{array}{l}\text { Speed } \\
\text { (mph) }\end{array}$ & $\begin{array}{l}\text { Elevation } \\
\text { (m) }\end{array}$ \\
\hline 161 & 13.14 & 200.0 \\
\hline 162 & 13.22 & 200.0 \\
\hline 163 & 13.21 & 200.0 \\
\hline 164 & 14.31 & 200.0 \\
\hline 165 & 14.45 & 200.0 \\
\hline 166 & 14.49 & 200.0 \\
\hline 167 & 15.10 & 200.0 \\
\hline 168 & 15.43 & 200.0 \\
\hline 169 & 16.60 & 200.0 \\
\hline 170 & 17.25 & 200.1 \\
\hline 171 & 17.71 & 200.2 \\
\hline 172 & 17.72 & 200.4 \\
\hline 173 & 18.00 & 200.6 \\
\hline 174 & 19.09 & 200.9 \\
\hline 175 & 19.15 & 201.1 \\
\hline 176 & 20.14 & 201.4 \\
\hline 177 & 20.91 & 201.7 \\
\hline 178 & 21.67 & 202.0 \\
\hline 179 & 21.70 & 202.3 \\
\hline 180 & 22.38 & 202.5 \\
\hline 181 & 23.05 & 202.7 \\
\hline 182 & 23.48 & 202.9 \\
\hline 183 & 23.65 & 203.0 \\
\hline 184 & 23.72 & 203.0 \\
\hline 185 & 24.80 & 203.0 \\
\hline 186 & 24.82 & 203.1 \\
\hline 187 & 26.26 & 203.1 \\
\hline 188 & 26.28 & 203.1 \\
\hline 189 & 27.54 & 203.2 \\
\hline 190 & 28.56 & 203.3 \\
\hline 191 & 28.60 & 203.3 \\
\hline 192 & 29.42 & 203.5 \\
\hline 193 & 30.18 & 203.6 \\
\hline 194 & 30.18 & 203.6 \\
\hline 195 & 30.67 & 203.8 \\
\hline 196 & 31.14 & 203.9 \\
\hline 197 & 31.38 & 204.1 \\
\hline 198 & 31.43 & 204.1 \\
\hline 199 & 31.98 & 203.7 \\
\hline 200 & 32.27 & 203.4 \\
\hline 201 & 33.01 & 203.2 \\
\hline 202 & 33.02 & 203.2 \\
\hline 203 & 33.38 & 202.9 \\
\hline 204 & 33.93 & 202.6 \\
\hline 205 & 35.00 & 202.3 \\
\hline 206 & 35.04 & 202.3 \\
\hline 207 & 36.16 & 202.1 \\
\hline 208 & 37.17 & 201.9 \\
\hline 209 & 37.19 & 201.9 \\
\hline 210 & 38.13 & 201.7 \\
\hline 211 & 38.50 & 201.6 \\
\hline 212 & 38.50 & 201.6 \\
\hline 213 & 38.85 & 201.6 \\
\hline 214 & 38.94 & 201.6 \\
\hline 215 & 39.20 & 201.3 \\
\hline 216 & 39.54 & 201.1 \\
\hline
\end{tabular}




\begin{tabular}{|c|c|c|}
\hline Time (s) & $\begin{array}{l}\text { Speed } \\
\text { (mph) }\end{array}$ & $\begin{array}{l}\text { Elevation } \\
(\mathrm{m})\end{array}$ \\
\hline 217 & 40.01 & 200.7 \\
\hline 218 & 40.27 & 200.3 \\
\hline 219 & 39.65 & 199.8 \\
\hline 220 & 39.63 & 199.4 \\
\hline 221 & 39.72 & 199.3 \\
\hline 222 & 40.09 & 199.3 \\
\hline 223 & 41.19 & 199.3 \\
\hline 224 & 41.49 & 199.4 \\
\hline 225 & 41.48 & 199.5 \\
\hline 226 & 41.49 & 199.5 \\
\hline 227 & 41.65 & 199.5 \\
\hline 228 & 42.01 & 199.5 \\
\hline 229 & 42.00 & 199.6 \\
\hline 230 & 42.09 & 199.8 \\
\hline 231 & 42.57 & 199.8 \\
\hline 232 & 43.17 & 199.8 \\
\hline 233 & 43.51 & 199.8 \\
\hline 234 & 43.76 & 199.8 \\
\hline 235 & 44.08 & 199.7 \\
\hline 236 & 44.12 & 199.5 \\
\hline 237 & 44.03 & 199.1 \\
\hline 238 & 43.94 & 198.8 \\
\hline 239 & 43.78 & 198.5 \\
\hline 240 & 43.58 & 198.3 \\
\hline 241 & 43.13 & 198.3 \\
\hline 242 & 43.27 & 198.0 \\
\hline 243 & 43.99 & 197.7 \\
\hline 244 & 44.55 & 197.5 \\
\hline 245 & 44.71 & 197.3 \\
\hline 246 & 45.47 & 197.0 \\
\hline 247 & 45.58 & 196.6 \\
\hline 248 & 45.58 & 196.3 \\
\hline 249 & 45.83 & 196.3 \\
\hline 250 & 46.03 & 196.3 \\
\hline 251 & 46.38 & 196.0 \\
\hline 252 & 46.61 & 195.8 \\
\hline 253 & 46.79 & 195.8 \\
\hline 254 & 47.02 & 195.6 \\
\hline 255 & 47.17 & 195.4 \\
\hline 256 & 47.16 & 195.4 \\
\hline 257 & 47.12 & 196.1 \\
\hline 258 & 46.96 & 196.7 \\
\hline 259 & 46.88 & 197.4 \\
\hline 260 & 47.78 & 197.3 \\
\hline 261 & 48.74 & 197.3 \\
\hline 262 & 49.02 & 197.3 \\
\hline 263 & 49.24 & 197.4 \\
\hline 264 & 49.08 & 197.3 \\
\hline 265 & 49.00 & 197.3 \\
\hline 266 & 48.82 & 197.3 \\
\hline 267 & 48.75 & 197.3 \\
\hline 268 & 48.84 & 197.3 \\
\hline 269 & 49.21 & 196.9 \\
\hline 270 & 49.75 & 196.5 \\
\hline 271 & 49.82 & 196.1 \\
\hline 272 & 49.96 & 196.1 \\
\hline
\end{tabular}

\begin{tabular}{|c|c|c|}
\hline Time (s) & $\begin{array}{l}\text { Speed } \\
\text { (mph) }\end{array}$ & $\begin{array}{l}\text { Elevation } \\
(\mathrm{m})\end{array}$ \\
\hline 273 & 50.15 & 195.9 \\
\hline 274 & 50.31 & 195.7 \\
\hline 275 & 50.81 & 195.7 \\
\hline 276 & 51.19 & 195.3 \\
\hline 277 & 51.47 & 194.9 \\
\hline 278 & 51.64 & 194.8 \\
\hline 279 & 51.54 & 195.0 \\
\hline 280 & 51.51 & 195.1 \\
\hline 281 & 51.49 & 195.5 \\
\hline 282 & 51.20 & 196.1 \\
\hline 283 & 50.88 & 196.5 \\
\hline 284 & 50.94 & 196.5 \\
\hline 285 & 50.97 & 195.7 \\
\hline 286 & 52.05 & 195.7 \\
\hline 287 & 52.96 & 195.7 \\
\hline 288 & 53.16 & 195.7 \\
\hline 289 & 53.07 & 195.7 \\
\hline 290 & 53.05 & 195.6 \\
\hline 291 & 53.17 & 195.6 \\
\hline 292 & 53.25 & 195.5 \\
\hline 293 & 53.34 & 195.5 \\
\hline 294 & 53.44 & 195.5 \\
\hline 295 & 53.47 & 195.4 \\
\hline 296 & 53.18 & 195.5 \\
\hline 297 & 51.92 & 196.1 \\
\hline 298 & 52.26 & 196.4 \\
\hline 299 & 52.48 & 196.3 \\
\hline 300 & 52.62 & 195.9 \\
\hline 301 & 52.46 & 196.0 \\
\hline 302 & 52.22 & 196.1 \\
\hline 303 & 52.00 & 196.4 \\
\hline 304 & 52.09 & 196.6 \\
\hline 305 & 52.17 & 196.6 \\
\hline 306 & 52.62 & 196.7 \\
\hline 307 & 53.03 & 196.9 \\
\hline 308 & 53.67 & 197.0 \\
\hline 309 & 54.33 & 197.1 \\
\hline 310 & 54.29 & 197.1 \\
\hline 311 & 54.42 & 196.1 \\
\hline 312 & 54.92 & 195.2 \\
\hline 313 & 55.01 & 194.3 \\
\hline 314 & 55.17 & 194.3 \\
\hline 315 & 55.06 & 194.3 \\
\hline 316 & 55.05 & 194.3 \\
\hline 317 & 55.02 & 194.2 \\
\hline 318 & 55.01 & 194.2 \\
\hline 319 & 55.14 & 194.2 \\
\hline 320 & 55.67 & 194.1 \\
\hline 321 & 55.98 & 194.0 \\
\hline 322 & 56.23 & 193.9 \\
\hline 323 & 55.85 & 193.9 \\
\hline 324 & 55.48 & 195.0 \\
\hline 325 & 55.02 & 196.1 \\
\hline 326 & 54.49 & 197.1 \\
\hline 327 & 54.42 & 197.1 \\
\hline 328 & 54.42 & 197.0 \\
\hline
\end{tabular}




\begin{tabular}{|c|c|c|}
\hline Time (s) & $\begin{array}{l}\text { Speed } \\
\text { (mph) }\end{array}$ & $\begin{array}{l}\text { Elevation } \\
(\mathrm{m})\end{array}$ \\
\hline 329 & 54.77 & 196.8 \\
\hline 330 & 54.91 & 196.6 \\
\hline 331 & 55.16 & 196.4 \\
\hline 332 & 55.33 & 196.1 \\
\hline 333 & 55.42 & 195.8 \\
\hline 334 & 55.69 & 195.5 \\
\hline 335 & 56.38 & 195.3 \\
\hline 336 & 57.10 & 195.1 \\
\hline 337 & 57.77 & 194.9 \\
\hline 338 & 58.41 & 194.7 \\
\hline 339 & 58.59 & 194.6 \\
\hline 340 & 58.65 & 194.4 \\
\hline 341 & 58.65 & 194.2 \\
\hline 342 & 58.62 & 194.0 \\
\hline 343 & 58.61 & 193.9 \\
\hline 344 & 58.57 & 193.7 \\
\hline 345 & 58.98 & 193.7 \\
\hline 346 & 59.44 & 193.8 \\
\hline 347 & 59.99 & 193.9 \\
\hline 348 & 59.99 & 193.9 \\
\hline 349 & 59.99 & 193.9 \\
\hline 350 & 59.64 & 193.9 \\
\hline 351 & 59.22 & 193.9 \\
\hline 352 & 58.79 & 193.9 \\
\hline 353 & 58.27 & 193.9 \\
\hline 354 & 57.98 & 193.9 \\
\hline 355 & 57.76 & 193.9 \\
\hline 356 & 57.05 & 193.9 \\
\hline 357 & 56.78 & 193.9 \\
\hline 358 & 56.67 & 193.9 \\
\hline 359 & 56.59 & 193.9 \\
\hline 360 & 56.60 & 194.0 \\
\hline 361 & 56.71 & 194.1 \\
\hline 362 & 56.71 & 194.2 \\
\hline 363 & 56.78 & 194.3 \\
\hline 364 & 57.06 & 194.4 \\
\hline 365 & 57.40 & 194.6 \\
\hline 366 & 57.59 & 194.8 \\
\hline 367 & 57.61 & 195.0 \\
\hline 368 & 57.80 & 195.1 \\
\hline 369 & 57.83 & 195.3 \\
\hline 370 & 57.84 & 195.4 \\
\hline 371 & 58.16 & 195.5 \\
\hline 372 & 58.71 & 195.7 \\
\hline 373 & 59.08 & 195.8 \\
\hline 374 & 59.44 & 195.8 \\
\hline 375 & 60.12 & 195.8 \\
\hline 376 & 60.01 & 195.8 \\
\hline 377 & 59.95 & 195.8 \\
\hline 378 & 59.91 & 195.8 \\
\hline 379 & 59.88 & 195.8 \\
\hline 380 & 59.81 & 195.8 \\
\hline 381 & 59.88 & 195.8 \\
\hline 382 & 59.91 & 196.0 \\
\hline 383 & 59.94 & 196.4 \\
\hline 384 & 59.95 & 196.7 \\
\hline
\end{tabular}

\begin{tabular}{|c|c|c|}
\hline Time (s) & $\begin{array}{l}\text { Speed } \\
\text { (mph) }\end{array}$ & $\begin{array}{l}\text { Elevation } \\
(\mathrm{m})\end{array}$ \\
\hline 385 & 60.10 & 196.9 \\
\hline 386 & 60.72 & 196.9 \\
\hline 387 & 61.34 & 196.9 \\
\hline 388 & 61.92 & 196.8 \\
\hline 389 & 62.48 & 196.7 \\
\hline 390 & 62.09 & 196.7 \\
\hline 391 & 61.73 & 197.3 \\
\hline 392 & 61.47 & 197.9 \\
\hline 393 & 61.88 & 197.9 \\
\hline 394 & 62.24 & 197.9 \\
\hline 395 & 62.32 & 197.8 \\
\hline 396 & 62.41 & 197.6 \\
\hline 397 & 63.02 & 197.2 \\
\hline 398 & 63.78 & 196.7 \\
\hline 399 & 64.63 & 196.2 \\
\hline 400 & 65.48 & 195.6 \\
\hline 401 & 66.09 & 195.1 \\
\hline 402 & 66.31 & 194.5 \\
\hline 403 & 66.35 & 194.0 \\
\hline 404 & 66.42 & 193.5 \\
\hline 405 & 66.49 & 193.2 \\
\hline 406 & 66.57 & 192.9 \\
\hline 407 & 66.66 & 192.7 \\
\hline 408 & 66.73 & 192.7 \\
\hline 409 & 66.80 & 192.7 \\
\hline 410 & 66.81 & 192.7 \\
\hline 411 & 66.75 & 192.7 \\
\hline 412 & 66.69 & 192.7 \\
\hline 413 & 66.63 & 192.7 \\
\hline 414 & 66.75 & 192.7 \\
\hline 415 & 66.81 & 192.7 \\
\hline 416 & 66.84 & 192.6 \\
\hline 417 & 66.88 & 192.4 \\
\hline 418 & 66.93 & 192.2 \\
\hline 419 & 67.01 & 192.0 \\
\hline 420 & 67.07 & 191.7 \\
\hline 421 & 67.16 & 191.4 \\
\hline 422 & 67.25 & 191.1 \\
\hline 423 & 67.32 & 190.8 \\
\hline 424 & 67.38 & 190.5 \\
\hline 425 & 67.38 & 190.3 \\
\hline 426 & 67.35 & 190.1 \\
\hline 427 & 67.35 & 190.0 \\
\hline 428 & 67.40 & 190.0 \\
\hline 429 & 67.48 & 190.0 \\
\hline 430 & 67.58 & 190.0 \\
\hline 431 & 67.67 & 190.0 \\
\hline 432 & 67.81 & 189.9 \\
\hline 433 & 67.89 & 189.8 \\
\hline 434 & 67.96 & 189.7 \\
\hline 435 & 68.00 & 189.6 \\
\hline 436 & 68.02 & 189.5 \\
\hline 437 & 68.09 & 189.5 \\
\hline 438 & 68.01 & 189.5 \\
\hline 439 & 68.02 & 189.5 \\
\hline 440 & 68.66 & 189.5 \\
\hline
\end{tabular}




\begin{tabular}{|c|c|c|}
\hline Time (s) & $\begin{array}{l}\text { Speed } \\
\text { (mph) }\end{array}$ & $\begin{array}{l}\text { Elevation } \\
(\mathrm{m})\end{array}$ \\
\hline 441 & 69.32 & 189.1 \\
\hline 442 & 69.91 & 188.7 \\
\hline 443 & 69.63 & 188.7 \\
\hline 444 & 69.44 & 188.8 \\
\hline 445 & 69.44 & 188.9 \\
\hline 446 & 69.44 & 189.2 \\
\hline 447 & 69.45 & 189.6 \\
\hline 448 & 69.63 & 190.1 \\
\hline 449 & 69.74 & 190.6 \\
\hline 450 & 69.79 & 191.0 \\
\hline 451 & 69.89 & 191.5 \\
\hline 452 & 69.94 & 191.9 \\
\hline 453 & 69.97 & 192.3 \\
\hline 454 & 70.00 & 192.7 \\
\hline 455 & 70.01 & 193.0 \\
\hline 456 & 70.00 & 193.3 \\
\hline 457 & 70.00 & 193.6 \\
\hline 458 & 70.00 & 193.9 \\
\hline 459 & 69.96 & 194.3 \\
\hline 460 & 69.91 & 194.6 \\
\hline 461 & 69.83 & 195.1 \\
\hline 462 & 69.65 & 195.5 \\
\hline 463 & 69.44 & 196.1 \\
\hline 464 & 69.29 & 196.7 \\
\hline 465 & 69.03 & 197.4 \\
\hline 466 & 68.82 & 198.1 \\
\hline 467 & 68.72 & 198.9 \\
\hline 468 & 68.43 & 199.7 \\
\hline 469 & 68.28 & 200.6 \\
\hline 470 & 68.00 & 201.5 \\
\hline 471 & 67.88 & 202.5 \\
\hline 472 & 67.62 & 203.4 \\
\hline 473 & 67.48 & 204.4 \\
\hline 474 & 67.24 & 205.4 \\
\hline 475 & 67.13 & 206.3 \\
\hline 476 & 66.93 & 207.2 \\
\hline 477 & 66.86 & 208.1 \\
\hline 478 & 66.83 & 208.9 \\
\hline 479 & 66.83 & 209.6 \\
\hline 480 & 66.86 & 210.3 \\
\hline 481 & 66.92 & 210.9 \\
\hline 482 & 67.07 & 211.4 \\
\hline 483 & 67.28 & 211.9 \\
\hline 484 & 67.50 & 212.3 \\
\hline 485 & 67.74 & 212.7 \\
\hline 486 & 68.13 & 212.9 \\
\hline 487 & 68.43 & 213.1 \\
\hline 488 & 68.92 & 213.3 \\
\hline 489 & 69.22 & 213.3 \\
\hline 490 & 69.43 & 213.3 \\
\hline 491 & 69.65 & 213.3 \\
\hline 492 & 69.80 & 213.3 \\
\hline 493 & 69.93 & 213.3 \\
\hline 494 & 70.19 & 213.3 \\
\hline 495 & 70.38 & 213.3 \\
\hline 496 & 70.44 & 213.3 \\
\hline
\end{tabular}

\begin{tabular}{|c|c|c|}
\hline Time (s) & $\begin{array}{l}\text { Speed } \\
\text { (mph) }\end{array}$ & $\begin{array}{l}\text { Elevation } \\
(\mathrm{m})\end{array}$ \\
\hline 497 & 70.59 & 213.3 \\
\hline 498 & 70.58 & 213.2 \\
\hline 499 & 70.58 & 213.1 \\
\hline 500 & 70.58 & 213.1 \\
\hline 501 & 70.64 & 213.0 \\
\hline 502 & 70.73 & 212.9 \\
\hline 503 & 70.81 & 212.9 \\
\hline 504 & 70.84 & 212.9 \\
\hline 505 & 70.87 & 212.9 \\
\hline 506 & 70.96 & 212.8 \\
\hline 507 & 71.05 & 212.7 \\
\hline 508 & 71.11 & 212.7 \\
\hline 509 & 71.14 & 212.6 \\
\hline 510 & 71.17 & 212.5 \\
\hline 511 & 71.17 & 212.4 \\
\hline 512 & 71.17 & 212.4 \\
\hline 513 & 71.17 & 212.4 \\
\hline 514 & 71.13 & 212.4 \\
\hline 515 & 71.08 & 212.4 \\
\hline 516 & 71.05 & 212.4 \\
\hline 517 & 71.09 & 212.4 \\
\hline 518 & 71.11 & 212.4 \\
\hline 519 & 71.23 & 212.4 \\
\hline 520 & 71.26 & 212.4 \\
\hline 521 & 71.26 & 212.4 \\
\hline 522 & 71.28 & 212.4 \\
\hline 523 & 71.21 & 212.4 \\
\hline 524 & 71.22 & 212.4 \\
\hline 525 & 71.18 & 212.4 \\
\hline 526 & 71.17 & 212.4 \\
\hline 527 & 71.40 & 212.4 \\
\hline 528 & 71.65 & 212.4 \\
\hline 529 & 72.06 & 212.4 \\
\hline 530 & 72.15 & 212.5 \\
\hline 531 & 72.30 & 212.5 \\
\hline 532 & 72.38 & 212.5 \\
\hline 533 & 72.31 & 212.5 \\
\hline 534 & 72.24 & 212.5 \\
\hline 535 & 72.18 & 212.5 \\
\hline 536 & 72.16 & 212.5 \\
\hline 537 & 72.68 & 212.6 \\
\hline 538 & 73.12 & 211.8 \\
\hline 539 & 73.41 & 211.0 \\
\hline 540 & 73.13 & 210.4 \\
\hline 541 & 72.79 & 210.0 \\
\hline 542 & 72.44 & 209.6 \\
\hline 543 & 72.00 & 209.4 \\
\hline 544 & 71.47 & 209.4 \\
\hline 545 & 71.19 & 209.4 \\
\hline 546 & 70.82 & 209.3 \\
\hline 547 & 70.47 & 209.3 \\
\hline 548 & 69.90 & 209.1 \\
\hline 549 & 69.23 & 209.0 \\
\hline 550 & 68.73 & 208.8 \\
\hline 551 & 68.05 & 208.5 \\
\hline 552 & 67.33 & 208.2 \\
\hline
\end{tabular}




\begin{tabular}{|c|c|c|}
\hline Time (s) & $\begin{array}{l}\text { Speed } \\
\text { (mph) }\end{array}$ & $\begin{array}{l}\text { Elevation } \\
(\mathrm{m})\end{array}$ \\
\hline 553 & 66.95 & 207.8 \\
\hline 554 & 66.94 & 207.4 \\
\hline 555 & 66.94 & 207.0 \\
\hline 556 & 66.97 & 206.5 \\
\hline 557 & 67.22 & 205.9 \\
\hline 558 & 67.38 & 205.4 \\
\hline 559 & 67.77 & 204.9 \\
\hline 560 & 67.99 & 204.4 \\
\hline 561 & 68.28 & 203.9 \\
\hline 562 & 68.41 & 203.5 \\
\hline 563 & 68.66 & 203.0 \\
\hline 564 & 68.79 & 202.6 \\
\hline 565 & 68.94 & 202.1 \\
\hline 566 & 69.01 & 201.7 \\
\hline 567 & 69.03 & 201.3 \\
\hline 568 & 69.21 & 200.9 \\
\hline 569 & 69.37 & 200.5 \\
\hline 570 & 69.40 & 200.1 \\
\hline 571 & 69.46 & 199.7 \\
\hline 572 & 69.64 & 199.3 \\
\hline 573 & 69.70 & 198.9 \\
\hline 574 & 69.91 & 198.5 \\
\hline 575 & 70.13 & 198.1 \\
\hline 576 & 70.35 & 197.6 \\
\hline 577 & 70.45 & 197.2 \\
\hline 578 & 70.51 & 196.7 \\
\hline 579 & 70.52 & 196.1 \\
\hline 580 & 70.52 & 195.5 \\
\hline 581 & 70.51 & 194.9 \\
\hline 582 & 70.49 & 194.1 \\
\hline 583 & 70.46 & 193.4 \\
\hline 584 & 70.35 & 192.4 \\
\hline 585 & 70.20 & 191.4 \\
\hline 586 & 70.03 & 190.3 \\
\hline 587 & 69.94 & 189.0 \\
\hline 588 & 69.85 & 187.7 \\
\hline 589 & 69.72 & 186.3 \\
\hline 590 & 69.63 & 184.7 \\
\hline 591 & 69.59 & 183.0 \\
\hline 592 & 69.55 & 181.3 \\
\hline 593 & 69.39 & 179.7 \\
\hline 594 & 69.36 & 178.1 \\
\hline 595 & 69.36 & 176.6 \\
\hline 596 & 69.36 & 175.2 \\
\hline 597 & 69.37 & 173.9 \\
\hline 598 & 69.38 & 172.6 \\
\hline 599 & 69.39 & 171.4 \\
\hline 600 & 69.41 & 170.1 \\
\hline 601 & 69.41 & 168.9 \\
\hline 602 & 69.42 & 167.7 \\
\hline 603 & 69.45 & 166.5 \\
\hline 604 & 69.46 & 165.3 \\
\hline 605 & 69.47 & 164.1 \\
\hline 606 & 69.50 & 163.0 \\
\hline 607 & 69.51 & 161.8 \\
\hline 608 & 69.48 & 160.6 \\
\hline
\end{tabular}

\begin{tabular}{|c|c|c|}
\hline Time (s) & $\begin{array}{l}\text { Speed } \\
\text { (mph) }\end{array}$ & $\begin{array}{l}\text { Elevation } \\
(\mathrm{m})\end{array}$ \\
\hline 609 & 69.44 & 159.5 \\
\hline 610 & 69.34 & 158.5 \\
\hline 611 & 69.28 & 157.6 \\
\hline 612 & 69.02 & 156.9 \\
\hline 613 & 68.77 & 156.2 \\
\hline 614 & 68.63 & 155.7 \\
\hline 615 & 68.50 & 155.3 \\
\hline 616 & 68.50 & 155.0 \\
\hline 617 & 68.50 & 154.8 \\
\hline 618 & 68.64 & 154.6 \\
\hline 619 & 68.87 & 154.5 \\
\hline 620 & 68.96 & 154.5 \\
\hline 621 & 69.11 & 154.4 \\
\hline 622 & 69.14 & 154.2 \\
\hline 623 & 69.19 & 154.1 \\
\hline 624 & 69.25 & 153.9 \\
\hline 625 & 69.27 & 153.8 \\
\hline 626 & 69.31 & 153.7 \\
\hline 627 & 69.44 & 153.6 \\
\hline 628 & 69.48 & 153.6 \\
\hline 629 & 69.53 & 153.6 \\
\hline 630 & 69.72 & 153.5 \\
\hline 631 & 70.00 & 153.3 \\
\hline 632 & 70.22 & 153.1 \\
\hline 633 & 70.49 & 152.9 \\
\hline 634 & 70.68 & 152.5 \\
\hline 635 & 70.90 & 152.1 \\
\hline 636 & 70.91 & 151.5 \\
\hline 637 & 70.94 & 150.9 \\
\hline 638 & 70.95 & 150.3 \\
\hline 639 & 70.98 & 149.6 \\
\hline 640 & 70.98 & 148.9 \\
\hline 641 & 70.97 & 148.1 \\
\hline 642 & 70.95 & 147.4 \\
\hline 643 & 70.96 & 146.6 \\
\hline 644 & 70.94 & 145.8 \\
\hline 645 & 70.89 & 145.0 \\
\hline 646 & 70.83 & 144.3 \\
\hline 647 & 70.78 & 143.6 \\
\hline 648 & 70.62 & 142.9 \\
\hline 649 & 70.55 & 142.9 \\
\hline 650 & 70.47 & 142.9 \\
\hline 651 & 70.22 & 142.2 \\
\hline 652 & 70.04 & 141.7 \\
\hline 653 & 69.87 & 141.2 \\
\hline 654 & 69.46 & 140.8 \\
\hline 655 & 69.30 & 140.5 \\
\hline 656 & 69.22 & 140.5 \\
\hline 657 & 69.14 & 140.5 \\
\hline 658 & 69.05 & 140.5 \\
\hline 659 & 68.97 & 140.5 \\
\hline 660 & 68.34 & 140.3 \\
\hline 661 & 67.57 & 140.2 \\
\hline 662 & 66.88 & 140.2 \\
\hline 663 & 66.70 & 140.2 \\
\hline 664 & 66.49 & 140.2 \\
\hline
\end{tabular}




\begin{tabular}{|c|c|c|}
\hline Time (s) & $\begin{array}{l}\text { Speed } \\
\text { (mph) }\end{array}$ & $\begin{array}{l}\text { Elevation } \\
(\mathrm{m})\end{array}$ \\
\hline 665 & 66.21 & 140.3 \\
\hline 666 & 66.08 & 140.4 \\
\hline 667 & 66.14 & 140.6 \\
\hline 668 & 66.13 & 140.9 \\
\hline 669 & 66.08 & 141.2 \\
\hline 670 & 66.04 & 141.6 \\
\hline 671 & 65.96 & 142.1 \\
\hline 672 & 65.92 & 142.4 \\
\hline 673 & 65.87 & 142.7 \\
\hline 674 & 65.84 & 142.9 \\
\hline 675 & 65.84 & 143.0 \\
\hline 676 & 65.79 & 143.1 \\
\hline 677 & 65.67 & 143.1 \\
\hline 678 & 65.70 & 143.1 \\
\hline 679 & 65.86 & 143.1 \\
\hline 680 & 65.84 & 143.1 \\
\hline 681 & 65.84 & 143.1 \\
\hline 682 & 65.76 & 143.1 \\
\hline 683 & 65.43 & 143.1 \\
\hline 684 & 65.00 & 143.1 \\
\hline 685 & 64.57 & 143.1 \\
\hline 686 & 64.49 & 143.1 \\
\hline 687 & 64.41 & 143.1 \\
\hline 688 & 64.32 & 143.1 \\
\hline 689 & 63.59 & 143.1 \\
\hline 690 & 62.85 & 143.1 \\
\hline 691 & 62.50 & 143.1 \\
\hline 692 & 62.31 & 143.0 \\
\hline 693 & 62.30 & 142.9 \\
\hline 694 & 62.63 & 142.3 \\
\hline 695 & 62.88 & 142.0 \\
\hline 696 & 63.18 & 141.7 \\
\hline 697 & 63.49 & 141.4 \\
\hline 698 & 63.75 & 141.0 \\
\hline 699 & 64.10 & 140.6 \\
\hline 700 & 64.33 & 140.1 \\
\hline 701 & 64.60 & 139.7 \\
\hline 702 & 64.87 & 139.3 \\
\hline 703 & 65.10 & 138.8 \\
\hline 704 & 65.33 & 138.4 \\
\hline 705 & 65.57 & 138.0 \\
\hline 706 & 65.65 & 137.6 \\
\hline 707 & 65.72 & 137.1 \\
\hline 708 & 65.81 & 136.7 \\
\hline 709 & 65.99 & 136.3 \\
\hline 710 & 66.08 & 136.0 \\
\hline 711 & 66.13 & 135.7 \\
\hline 712 & 66.21 & 135.4 \\
\hline 713 & 66.37 & 135.2 \\
\hline 714 & 66.20 & 135.2 \\
\hline 715 & 66.05 & 135.5 \\
\hline 716 & 65.90 & 136.0 \\
\hline 717 & 65.66 & 136.7 \\
\hline 718 & 65.58 & 137.4 \\
\hline 719 & 65.32 & 138.0 \\
\hline 720 & 65.06 & 138.6 \\
\hline
\end{tabular}

\begin{tabular}{|c|c|c|}
\hline Time (s) & $\begin{array}{l}\text { Speed } \\
\text { (mph) }\end{array}$ & $\begin{array}{l}\text { Elevation } \\
\text { (m) }\end{array}$ \\
\hline 721 & 65.06 & 139.1 \\
\hline 722 & 65.14 & 139.3 \\
\hline 723 & 65.22 & 139.5 \\
\hline 724 & 65.30 & 139.7 \\
\hline 725 & 65.36 & 139.8 \\
\hline 726 & 65.72 & 140.1 \\
\hline 727 & 65.99 & 140.4 \\
\hline 728 & 66.32 & 140.4 \\
\hline 729 & 66.58 & 140.4 \\
\hline 730 & 66.86 & 140.3 \\
\hline 731 & 67.43 & 140.2 \\
\hline 732 & 67.89 & 140.0 \\
\hline 733 & 68.44 & 140.0 \\
\hline 734 & 67.99 & 140.2 \\
\hline 735 & 67.79 & 140.9 \\
\hline 736 & 67.52 & 141.8 \\
\hline 737 & 67.33 & 142.6 \\
\hline 738 & 66.97 & 143.1 \\
\hline 739 & 66.89 & 143.1 \\
\hline 740 & 66.86 & 143.0 \\
\hline 741 & 66.74 & 142.6 \\
\hline 742 & 66.39 & 142.1 \\
\hline 743 & 66.14 & 141.7 \\
\hline 744 & 66.02 & 141.4 \\
\hline 745 & 65.91 & 141.1 \\
\hline 746 & 65.62 & 140.9 \\
\hline 747 & 64.86 & 140.7 \\
\hline 748 & 64.69 & 140.7 \\
\hline 749 & 64.74 & 140.7 \\
\hline 750 & 64.75 & 140.8 \\
\hline 751 & 64.67 & 141.0 \\
\hline 752 & 64.50 & 141.1 \\
\hline 753 & 64.48 & 141.2 \\
\hline 754 & 64.55 & 141.3 \\
\hline 755 & 64.72 & 141.4 \\
\hline 756 & 64.89 & 141.4 \\
\hline 757 & 65.32 & 141.5 \\
\hline 758 & 65.66 & 141.5 \\
\hline 759 & 65.38 & 141.7 \\
\hline 760 & 65.14 & 142.4 \\
\hline 761 & 64.86 & 143.4 \\
\hline 762 & 64.62 & 144.4 \\
\hline 763 & 64.38 & 145.4 \\
\hline 764 & 64.14 & 146.3 \\
\hline 765 & 63.85 & 147.2 \\
\hline 766 & 63.62 & 148.0 \\
\hline 767 & 63.50 & 148.7 \\
\hline 768 & 63.42 & 149.4 \\
\hline 769 & 63.33 & 150.0 \\
\hline 770 & 63.32 & 150.5 \\
\hline 771 & 63.33 & 150.9 \\
\hline 772 & 63.35 & 151.3 \\
\hline 773 & 63.36 & 151.5 \\
\hline 774 & 63.47 & 151.7 \\
\hline 775 & 63.56 & 151.8 \\
\hline 776 & 63.47 & 151.8 \\
\hline
\end{tabular}




\begin{tabular}{|c|c|c|}
\hline Time (s) & $\begin{array}{l}\text { Speed } \\
\text { (mph) }\end{array}$ & $\begin{array}{l}\text { Elevation } \\
(\mathrm{m})\end{array}$ \\
\hline 777 & 63.44 & 151.8 \\
\hline 778 & 63.44 & 151.9 \\
\hline 779 & 63.39 & 152.0 \\
\hline 780 & 63.35 & 152.1 \\
\hline 781 & 63.31 & 152.4 \\
\hline 782 & 63.08 & 152.7 \\
\hline 783 & 62.81 & 153.1 \\
\hline 784 & 62.56 & 153.6 \\
\hline 785 & 62.30 & 154.1 \\
\hline 786 & 62.18 & 154.8 \\
\hline 787 & 61.96 & 155.5 \\
\hline 788 & 61.79 & 156.3 \\
\hline 789 & 61.70 & 157.1 \\
\hline 790 & 61.57 & 157.9 \\
\hline 791 & 61.44 & 158.7 \\
\hline 792 & 61.35 & 159.5 \\
\hline 793 & 61.23 & 160.3 \\
\hline 794 & 61.14 & 161.1 \\
\hline 795 & 60.97 & 161.9 \\
\hline 796 & 60.91 & 162.6 \\
\hline 797 & 60.99 & 163.2 \\
\hline 798 & 61.17 & 163.6 \\
\hline 799 & 61.51 & 164.0 \\
\hline 800 & 61.77 & 164.2 \\
\hline 801 & 62.46 & 164.2 \\
\hline 802 & 62.77 & 164.2 \\
\hline 803 & 62.86 & 163.9 \\
\hline 804 & 62.97 & 163.0 \\
\hline 805 & 63.12 & 162.2 \\
\hline 806 & 63.22 & 161.3 \\
\hline 807 & 63.71 & 160.4 \\
\hline 808 & 63.83 & 159.5 \\
\hline 809 & 63.91 & 158.6 \\
\hline 810 & 63.91 & 157.7 \\
\hline 811 & 63.77 & 157.2 \\
\hline 812 & 63.65 & 157.1 \\
\hline 813 & 63.74 & 157.1 \\
\hline 814 & 63.91 & 157.1 \\
\hline 815 & 64.04 & 157.1 \\
\hline 816 & 64.16 & 157.0 \\
\hline 817 & 64.24 & 156.9 \\
\hline 818 & 64.30 & 156.7 \\
\hline 819 & 64.35 & 156.5 \\
\hline 820 & 64.40 & 156.3 \\
\hline 821 & 64.42 & 156.0 \\
\hline 822 & 64.43 & 155.8 \\
\hline 823 & 64.43 & 155.5 \\
\hline 824 & 64.42 & 155.2 \\
\hline 825 & 64.42 & 154.9 \\
\hline 826 & 64.42 & 154.6 \\
\hline 827 & 64.41 & 154.3 \\
\hline 828 & 64.39 & 153.9 \\
\hline 829 & 64.36 & 153.6 \\
\hline 830 & 64.31 & 153.3 \\
\hline 831 & 64.26 & 153.0 \\
\hline 832 & 64.24 & 152.7 \\
\hline
\end{tabular}

\begin{tabular}{|c|c|c|}
\hline Time (s) & $\begin{array}{l}\text { Speed } \\
\text { (mph) }\end{array}$ & $\begin{array}{l}\text { Elevation } \\
(\mathrm{m})\end{array}$ \\
\hline 833 & 64.20 & 152.4 \\
\hline 834 & 64.13 & 152.2 \\
\hline 835 & 64.04 & 151.9 \\
\hline 836 & 63.90 & 151.8 \\
\hline 837 & 63.84 & 151.6 \\
\hline 838 & 63.76 & 151.4 \\
\hline 839 & 63.52 & 151.3 \\
\hline 840 & 62.85 & 151.2 \\
\hline 841 & 62.51 & 151.2 \\
\hline 842 & 62.40 & 151.1 \\
\hline 843 & 62.29 & 151.1 \\
\hline 844 & 62.22 & 151.2 \\
\hline 845 & 62.20 & 151.3 \\
\hline 846 & 62.28 & 151.5 \\
\hline 847 & 62.15 & 151.5 \\
\hline 848 & 62.13 & 151.7 \\
\hline 849 & 62.12 & 152.0 \\
\hline 850 & 62.12 & 152.2 \\
\hline 851 & 62.12 & 152.5 \\
\hline 852 & 62.20 & 152.8 \\
\hline 853 & 62.28 & 152.8 \\
\hline 854 & 62.47 & 152.8 \\
\hline 855 & 62.60 & 152.8 \\
\hline 856 & 63.04 & 152.9 \\
\hline 857 & 63.49 & 153.0 \\
\hline 858 & 63.57 & 153.0 \\
\hline 859 & 64.19 & 153.0 \\
\hline 860 & 64.63 & 153.0 \\
\hline 861 & 64.89 & 153.1 \\
\hline 862 & 65.07 & 153.2 \\
\hline 863 & 65.15 & 153.2 \\
\hline 864 & 65.24 & 153.3 \\
\hline 865 & 65.32 & 153.3 \\
\hline 866 & 65.40 & 153.3 \\
\hline 867 & 65.40 & 153.3 \\
\hline 868 & 65.39 & 153.3 \\
\hline 869 & 65.38 & 153.3 \\
\hline 870 & 65.36 & 153.3 \\
\hline 871 & 64.81 & 153.2 \\
\hline 872 & 64.47 & 153.2 \\
\hline 873 & 64.28 & 153.2 \\
\hline 874 & 64.01 & 153.2 \\
\hline 875 & 63.72 & 153.7 \\
\hline 876 & 63.31 & 154.3 \\
\hline 877 & 63.08 & 154.9 \\
\hline 878 & 63.04 & 154.9 \\
\hline 879 & 63.05 & 154.8 \\
\hline 880 & 63.05 & 154.7 \\
\hline 881 & 63.21 & 154.7 \\
\hline 882 & 63.30 & 154.7 \\
\hline 883 & 63.56 & 154.7 \\
\hline 884 & 64.28 & 154.7 \\
\hline 885 & 64.64 & 154.7 \\
\hline 886 & 64.86 & 154.8 \\
\hline 887 & 65.01 & 154.9 \\
\hline 888 & 65.07 & 154.9 \\
\hline
\end{tabular}




\begin{tabular}{|c|c|c|}
\hline Time (s) & $\begin{array}{l}\text { Speed } \\
\text { (mph) }\end{array}$ & $\begin{array}{l}\text { Elevation } \\
(\mathrm{m})\end{array}$ \\
\hline 889 & 65.15 & 155.0 \\
\hline 890 & 65.21 & 155.1 \\
\hline 891 & 65.07 & 155.1 \\
\hline 892 & 64.93 & 155.1 \\
\hline 893 & 64.79 & 155.1 \\
\hline 894 & 64.65 & 155.2 \\
\hline 895 & 64.62 & 155.3 \\
\hline 896 & 64.63 & 155.5 \\
\hline 897 & 64.68 & 155.7 \\
\hline 898 & 64.75 & 155.8 \\
\hline 899 & 64.83 & 156.0 \\
\hline 900 & 64.91 & 156.2 \\
\hline 901 & 64.99 & 156.3 \\
\hline 902 & 65.08 & 156.4 \\
\hline 903 & 65.20 & 156.5 \\
\hline 904 & 65.75 & 156.5 \\
\hline 905 & 66.20 & 156.3 \\
\hline 906 & 66.38 & 156.2 \\
\hline 907 & 66.50 & 156.1 \\
\hline 908 & 66.32 & 156.1 \\
\hline 909 & 65.68 & 156.1 \\
\hline 910 & 65.55 & 156.1 \\
\hline 911 & 65.40 & 156.1 \\
\hline 912 & 65.35 & 156.0 \\
\hline 913 & 65.24 & 156.0 \\
\hline 914 & 65.23 & 156.0 \\
\hline 915 & 65.23 & 155.9 \\
\hline 916 & 65.23 & 155.9 \\
\hline 917 & 65.33 & 155.8 \\
\hline 918 & 65.50 & 155.8 \\
\hline 919 & 65.97 & 155.8 \\
\hline 920 & 66.34 & 155.8 \\
\hline 921 & 66.71 & 155.9 \\
\hline 922 & 67.00 & 155.9 \\
\hline 923 & 67.39 & 156.0 \\
\hline 924 & 67.39 & 156.0 \\
\hline 925 & 67.30 & 156.0 \\
\hline 926 & 67.35 & 156.0 \\
\hline 927 & 67.50 & 156.0 \\
\hline 928 & 67.72 & 156.2 \\
\hline 929 & 67.96 & 156.3 \\
\hline 930 & 68.09 & 156.6 \\
\hline 931 & 68.13 & 156.9 \\
\hline 932 & 68.14 & 157.3 \\
\hline 933 & 68.14 & 157.8 \\
\hline 934 & 68.06 & 158.4 \\
\hline 935 & 67.98 & 159.1 \\
\hline 936 & 67.92 & 159.8 \\
\hline 937 & 67.84 & 160.5 \\
\hline 938 & 67.78 & 161.3 \\
\hline 939 & 67.72 & 162.0 \\
\hline 940 & 67.66 & 162.8 \\
\hline 941 & 67.59 & 163.5 \\
\hline 942 & 67.53 & 164.2 \\
\hline 943 & 67.37 & 164.8 \\
\hline 944 & 67.24 & 165.5 \\
\hline
\end{tabular}

\begin{tabular}{|c|c|c|}
\hline Time (s) & $\begin{array}{l}\text { Speed } \\
\text { (mph) }\end{array}$ & $\begin{array}{l}\text { Elevation } \\
(\mathrm{m})\end{array}$ \\
\hline 945 & 67.16 & 166.1 \\
\hline 946 & 66.91 & 166.7 \\
\hline 947 & 66.83 & 167.2 \\
\hline 948 & 66.76 & 167.7 \\
\hline 949 & 66.76 & 168.1 \\
\hline 950 & 66.77 & 168.7 \\
\hline 951 & 66.87 & 168.9 \\
\hline 952 & 67.10 & 169.0 \\
\hline 953 & 67.39 & 169.0 \\
\hline 954 & 67.05 & 169.0 \\
\hline 955 & 66.38 & 169.0 \\
\hline 956 & 66.19 & 169.1 \\
\hline 957 & 66.07 & 169.1 \\
\hline 958 & 65.70 & 169.2 \\
\hline 959 & 65.69 & 169.2 \\
\hline 960 & 65.69 & 169.3 \\
\hline 961 & 65.75 & 169.3 \\
\hline 962 & 66.65 & 169.1 \\
\hline 963 & 67.53 & 168.8 \\
\hline 964 & 67.88 & 168.8 \\
\hline 965 & 67.88 & 168.8 \\
\hline 966 & 67.88 & 169.1 \\
\hline 967 & 67.35 & 169.5 \\
\hline 968 & 66.76 & 170.1 \\
\hline 969 & 66.26 & 170.8 \\
\hline 970 & 65.71 & 171.7 \\
\hline 971 & 65.19 & 172.6 \\
\hline 972 & 64.72 & 173.6 \\
\hline 973 & 64.35 & 174.4 \\
\hline 974 & 64.11 & 175.3 \\
\hline 975 & 64.01 & 176.0 \\
\hline 976 & 63.98 & 176.7 \\
\hline 977 & 63.98 & 177.3 \\
\hline 978 & 63.99 & 177.8 \\
\hline 979 & 64.09 & 178.2 \\
\hline 980 & 64.11 & 178.5 \\
\hline 981 & 64.14 & 178.7 \\
\hline 982 & 64.31 & 178.8 \\
\hline 983 & 64.57 & 179.0 \\
\hline 984 & 64.88 & 179.0 \\
\hline 985 & 64.53 & 178.9 \\
\hline 986 & 63.62 & 178.8 \\
\hline 987 & 62.60 & 178.7 \\
\hline 988 & 62.77 & 178.7 \\
\hline 989 & 62.99 & 178.7 \\
\hline 990 & 63.57 & 178.7 \\
\hline 991 & 64.24 & 178.6 \\
\hline 992 & 64.78 & 178.6 \\
\hline 993 & 65.22 & 178.5 \\
\hline 994 & 65.46 & 178.4 \\
\hline 995 & 65.62 & 178.2 \\
\hline 996 & 66.27 & 178.1 \\
\hline 997 & 66.92 & 177.9 \\
\hline 998 & 67.59 & 177.7 \\
\hline 999 & 68.07 & 177.5 \\
\hline 1000 & 68.19 & 177.4 \\
\hline
\end{tabular}




\begin{tabular}{|c|c|c|}
\hline Time (s) & $\begin{array}{l}\text { Speed } \\
\text { (mph) }\end{array}$ & $\begin{array}{l}\text { Elevation } \\
(\mathrm{m})\end{array}$ \\
\hline 1001 & 68.35 & 177.2 \\
\hline 1002 & 68.41 & 177.0 \\
\hline 1003 & 68.48 & 176.9 \\
\hline 1004 & 68.63 & 176.7 \\
\hline 1005 & 68.77 & 176.6 \\
\hline 1006 & 68.86 & 176.5 \\
\hline 1007 & 68.94 & 176.3 \\
\hline 1008 & 69.47 & 176.2 \\
\hline 1009 & 70.06 & 176.1 \\
\hline 1010 & 70.59 & 176.0 \\
\hline 1011 & 71.16 & 175.8 \\
\hline 1012 & 71.68 & 175.6 \\
\hline 1013 & 72.27 & 175.5 \\
\hline 1014 & 72.33 & 175.3 \\
\hline 1015 & 72.33 & 175.1 \\
\hline 1016 & 72.34 & 174.9 \\
\hline 1017 & 72.34 & 174.8 \\
\hline 1018 & 72.34 & 174.6 \\
\hline 1019 & 72.33 & 174.4 \\
\hline 1020 & 72.34 & 174.3 \\
\hline 1021 & 72.34 & 174.1 \\
\hline 1022 & 72.34 & 173.9 \\
\hline 1023 & 72.33 & 173.7 \\
\hline 1024 & 72.32 & 173.6 \\
\hline 1025 & 72.26 & 173.4 \\
\hline 1026 & 72.15 & 173.3 \\
\hline 1027 & 72.05 & 173.1 \\
\hline 1028 & 72.01 & 173.0 \\
\hline 1029 & 71.84 & 172.9 \\
\hline 1030 & 71.57 & 172.9 \\
\hline 1031 & 71.49 & 172.9 \\
\hline 1032 & 71.43 & 172.9 \\
\hline 1033 & 71.74 & 172.9 \\
\hline 1034 & 71.91 & 173.0 \\
\hline 1035 & 71.98 & 173.0 \\
\hline 1036 & 72.43 & 173.0 \\
\hline 1037 & 72.73 & 172.9 \\
\hline 1038 & 73.00 & 172.6 \\
\hline 1039 & 73.04 & 172.3 \\
\hline 1040 & 73.11 & 172.2 \\
\hline 1041 & 73.29 & 171.7 \\
\hline 1042 & 73.46 & 171.0 \\
\hline 1043 & 73.81 & 170.2 \\
\hline 1044 & 74.65 & 169.2 \\
\hline 1045 & 74.98 & 168.2 \\
\hline 1046 & 75.42 & 167.2 \\
\hline 1047 & 75.51 & 166.4 \\
\hline 1048 & 75.59 & 165.8 \\
\hline 1049 & 75.67 & 165.3 \\
\hline 1050 & 75.58 & 165.3 \\
\hline 1051 & 75.50 & 165.3 \\
\hline 1052 & 75.54 & 165.3 \\
\hline 1053 & 75.67 & 165.3 \\
\hline 1054 & 75.91 & 164.7 \\
\hline 1055 & 76.41 & 164.1 \\
\hline 1056 & 76.56 & 163.5 \\
\hline
\end{tabular}

\begin{tabular}{|c|c|c|}
\hline Time (s) & $\begin{array}{l}\text { Speed } \\
\text { (mph) }\end{array}$ & $\begin{array}{l}\text { Elevation } \\
\text { (m) }\end{array}$ \\
\hline 1057 & 76.54 & 162.8 \\
\hline 1058 & 76.51 & 162.8 \\
\hline 1059 & 76.53 & 162.8 \\
\hline 1060 & 76.62 & 162.2 \\
\hline 1061 & 76.81 & 161.4 \\
\hline 1062 & 76.88 & 160.7 \\
\hline 1063 & 76.97 & 159.9 \\
\hline 1064 & 77.06 & 158.9 \\
\hline 1065 & 77.05 & 158.9 \\
\hline 1066 & 77.19 & 158.1 \\
\hline 1067 & 77.39 & 157.3 \\
\hline 1068 & 78.01 & 156.7 \\
\hline 1069 & 78.03 & 156.3 \\
\hline 1070 & 77.24 & 155.9 \\
\hline 1071 & 76.24 & 155.6 \\
\hline 1072 & 76.07 & 155.4 \\
\hline 1073 & 75.98 & 155.4 \\
\hline 1074 & 75.90 & 155.4 \\
\hline 1075 & 75.82 & 155.4 \\
\hline 1076 & 75.62 & 155.4 \\
\hline 1077 & 75.28 & 155.4 \\
\hline 1078 & 74.85 & 155.4 \\
\hline 1079 & 74.77 & 155.4 \\
\hline 1080 & 74.74 & 155.4 \\
\hline 1081 & 74.76 & 155.4 \\
\hline 1082 & 74.43 & 155.6 \\
\hline 1083 & 73.99 & 155.9 \\
\hline 1084 & 73.93 & 156.0 \\
\hline 1085 & 73.78 & 156.1 \\
\hline 1086 & 73.73 & 156.1 \\
\hline 1087 & 73.74 & 156.2 \\
\hline 1088 & 73.77 & 156.3 \\
\hline 1089 & 73.79 & 156.3 \\
\hline 1090 & 73.82 & 156.5 \\
\hline 1091 & 73.83 & 156.5 \\
\hline 1092 & 73.85 & 156.7 \\
\hline 1093 & 73.87 & 157.0 \\
\hline 1094 & 73.87 & 157.0 \\
\hline 1095 & 73.95 & 157.3 \\
\hline 1096 & 74.02 & 157.4 \\
\hline 1097 & 74.08 & 157.5 \\
\hline 1098 & 73.92 & 157.6 \\
\hline 1099 & 74.00 & 157.7 \\
\hline 1100 & 74.21 & 157.9 \\
\hline 1101 & 74.41 & 158.1 \\
\hline 1102 & 74.49 & 158.3 \\
\hline 1103 & 74.58 & 158.5 \\
\hline 1104 & 74.66 & 158.6 \\
\hline 1105 & 74.74 & 158.7 \\
\hline 1106 & 74.82 & 158.7 \\
\hline 1107 & 74.98 & 158.7 \\
\hline 1108 & 75.13 & 158.7 \\
\hline 1109 & 75.21 & 158.6 \\
\hline 1110 & 75.34 & 158.5 \\
\hline 1111 & 75.43 & 158.4 \\
\hline 1112 & 75.49 & 158.1 \\
\hline
\end{tabular}




\begin{tabular}{|c|c|c|}
\hline Time (s) & $\begin{array}{l}\text { Speed } \\
\text { (mph) }\end{array}$ & $\begin{array}{l}\text { Elevation } \\
(\mathrm{m})\end{array}$ \\
\hline 1113 & 75.48 & 157.9 \\
\hline 1114 & 75.30 & 157.6 \\
\hline 1115 & 75.15 & 157.3 \\
\hline 1116 & 74.96 & 157.1 \\
\hline 1117 & 74.90 & 156.8 \\
\hline 1118 & 74.88 & 156.5 \\
\hline 1119 & 74.88 & 156.2 \\
\hline 1120 & 74.86 & 155.9 \\
\hline 1121 & 74.84 & 155.7 \\
\hline 1122 & 74.84 & 155.4 \\
\hline 1123 & 74.87 & 155.1 \\
\hline 1124 & 74.88 & 154.8 \\
\hline 1125 & 74.90 & 154.5 \\
\hline 1126 & 74.90 & 154.2 \\
\hline 1127 & 74.88 & 154.0 \\
\hline 1128 & 74.79 & 153.8 \\
\hline 1129 & 74.65 & 153.7 \\
\hline 1130 & 74.47 & 153.6 \\
\hline 1131 & 74.38 & 153.5 \\
\hline 1132 & 74.25 & 153.5 \\
\hline 1133 & 74.07 & 153.5 \\
\hline 1134 & 74.00 & 153.5 \\
\hline 1135 & 73.91 & 153.5 \\
\hline 1136 & 73.82 & 153.5 \\
\hline 1137 & 73.62 & 153.5 \\
\hline 1138 & 73.37 & 153.6 \\
\hline 1139 & 73.34 & 153.7 \\
\hline 1140 & 73.34 & 153.8 \\
\hline 1141 & 73.34 & 153.8 \\
\hline 1142 & 73.51 & 153.8 \\
\hline 1143 & 73.59 & 153.8 \\
\hline 1144 & 73.09 & 153.8 \\
\hline 1145 & 72.56 & 154.0 \\
\hline 1146 & 72.49 & 154.4 \\
\hline 1147 & 72.22 & 154.9 \\
\hline 1148 & 71.98 & 155.6 \\
\hline 1149 & 71.87 & 156.4 \\
\hline 1150 & 71.47 & 157.2 \\
\hline 1151 & 71.09 & 158.2 \\
\hline 1152 & 70.68 & 159.2 \\
\hline 1153 & 70.35 & 160.4 \\
\hline 1154 & 70.00 & 161.5 \\
\hline 1155 & 69.68 & 162.7 \\
\hline 1156 & 69.25 & 163.9 \\
\hline 1157 & 68.97 & 165.0 \\
\hline 1158 & 68.69 & 166.0 \\
\hline 1159 & 68.54 & 167.0 \\
\hline 1160 & 68.54 & 167.8 \\
\hline 1161 & 68.54 & 168.4 \\
\hline 1162 & 68.55 & 168.8 \\
\hline 1163 & 68.98 & 169.2 \\
\hline 1164 & 67.89 & 169.2 \\
\hline 1165 & 68.09 & 169.3 \\
\hline 1166 & 68.46 & 169.8 \\
\hline 1167 & 68.65 & 170.3 \\
\hline 1168 & 69.01 & 170.7 \\
\hline
\end{tabular}

\begin{tabular}{|c|c|c|}
\hline Time (s) & $\begin{array}{l}\text { Speed } \\
\text { (mph) }\end{array}$ & $\begin{array}{l}\text { Elevation } \\
\text { (m) }\end{array}$ \\
\hline 1169 & 69.28 & 171.1 \\
\hline 1170 & 69.58 & 171.5 \\
\hline 1171 & 70.00 & 171.8 \\
\hline 1172 & 69.94 & 171.8 \\
\hline 1173 & 69.94 & 171.4 \\
\hline 1174 & 69.93 & 171.2 \\
\hline 1175 & 69.91 & 170.9 \\
\hline 1176 & 69.88 & 170.5 \\
\hline 1177 & 69.89 & 170.2 \\
\hline 1178 & 69.87 & 170.0 \\
\hline 1179 & 69.86 & 169.7 \\
\hline 1180 & 69.85 & 169.5 \\
\hline 1181 & 69.82 & 169.3 \\
\hline 1182 & 69.78 & 169.0 \\
\hline 1183 & 69.76 & 168.8 \\
\hline 1184 & 69.79 & 168.5 \\
\hline 1185 & 69.83 & 168.3 \\
\hline 1186 & 69.90 & 168.0 \\
\hline 1187 & 69.93 & 167.6 \\
\hline 1188 & 69.97 & 167.2 \\
\hline 1189 & 69.93 & 166.8 \\
\hline 1190 & 69.92 & 166.3 \\
\hline 1191 & 69.89 & 165.8 \\
\hline 1192 & 69.88 & 165.2 \\
\hline 1193 & 69.93 & 165.2 \\
\hline 1194 & 70.33 & 165.3 \\
\hline 1195 & 70.67 & 165.3 \\
\hline 1196 & 70.99 & 165.4 \\
\hline 1197 & 71.41 & 165.6 \\
\hline 1198 & 71.62 & 165.8 \\
\hline 1199 & 71.70 & 166.0 \\
\hline 1200 & 71.79 & 166.2 \\
\hline 1201 & 71.83 & 166.4 \\
\hline 1202 & 71.91 & 166.6 \\
\hline 1203 & 71.98 & 166.8 \\
\hline 1204 & 72.01 & 167.1 \\
\hline 1205 & 72.05 & 167.2 \\
\hline 1206 & 72.05 & 167.4 \\
\hline 1207 & 72.04 & 167.6 \\
\hline 1208 & 71.99 & 167.8 \\
\hline 1209 & 71.82 & 168.0 \\
\hline 1210 & 71.56 & 168.1 \\
\hline 1211 & 71.22 & 168.3 \\
\hline 1212 & 70.95 & 168.3 \\
\hline 1213 & 70.69 & 168.4 \\
\hline 1214 & 70.60 & 168.4 \\
\hline 1215 & 70.53 & 168.4 \\
\hline 1216 & 70.47 & 168.5 \\
\hline 1217 & 70.51 & 168.7 \\
\hline 1218 & 70.47 & 168.7 \\
\hline 1219 & 70.46 & 168.7 \\
\hline 1220 & 70.59 & 168.9 \\
\hline 1221 & 70.68 & 169.4 \\
\hline 1222 & 70.77 & 169.8 \\
\hline 1223 & 70.85 & 170.2 \\
\hline 1224 & 70.98 & 170.6 \\
\hline
\end{tabular}




\begin{tabular}{|c|c|c|}
\hline Time (s) & $\begin{array}{l}\text { Speed } \\
\text { (mph) }\end{array}$ & $\begin{array}{l}\text { Elevation } \\
(\mathrm{m})\end{array}$ \\
\hline 1225 & 71.11 & 171.1 \\
\hline 1226 & 71.28 & 171.5 \\
\hline 1227 & 71.40 & 171.9 \\
\hline 1228 & 71.54 & 172.4 \\
\hline 1229 & 71.61 & 173.2 \\
\hline 1230 & 71.62 & 173.6 \\
\hline 1231 & 71.59 & 174.0 \\
\hline 1232 & 71.58 & 174.4 \\
\hline 1233 & 71.64 & 175.0 \\
\hline 1234 & 71.69 & 175.3 \\
\hline 1235 & 71.76 & 175.6 \\
\hline 1236 & 71.87 & 175.8 \\
\hline 1237 & 71.92 & 176.1 \\
\hline 1238 & 71.92 & 176.4 \\
\hline 1239 & 71.86 & 176.8 \\
\hline 1240 & 71.80 & 177.2 \\
\hline 1241 & 71.72 & 177.7 \\
\hline 1242 & 71.46 & 178.2 \\
\hline 1243 & 71.12 & 178.8 \\
\hline 1244 & 70.94 & 179.4 \\
\hline 1245 & 70.64 & 180.0 \\
\hline 1246 & 70.40 & 180.7 \\
\hline 1247 & 69.88 & 181.4 \\
\hline 1248 & 69.62 & 182.1 \\
\hline 1249 & 69.53 & 182.8 \\
\hline 1250 & 69.26 & 183.5 \\
\hline 1251 & 68.90 & 184.1 \\
\hline 1252 & 68.52 & 184.8 \\
\hline 1253 & 68.33 & 185.4 \\
\hline 1254 & 68.08 & 185.9 \\
\hline 1255 & 67.91 & 186.5 \\
\hline 1256 & 67.87 & 187.0 \\
\hline 1257 & 67.86 & 187.6 \\
\hline 1258 & 67.76 & 188.1 \\
\hline 1259 & 67.76 & 188.6 \\
\hline 1260 & 67.79 & 189.1 \\
\hline 1261 & 67.85 & 189.6 \\
\hline 1262 & 67.92 & 190.1 \\
\hline 1263 & 67.95 & 190.6 \\
\hline 1264 & 67.96 & 191.0 \\
\hline 1265 & 67.91 & 191.4 \\
\hline 1266 & 67.85 & 191.6 \\
\hline 1267 & 67.79 & 191.7 \\
\hline 1268 & 67.74 & 191.7 \\
\hline 1269 & 67.80 & 191.7 \\
\hline 1270 & 68.07 & 191.8 \\
\hline 1271 & 68.11 & 192.0 \\
\hline 1272 & 68.15 & 192.2 \\
\hline 1273 & 68.18 & 192.4 \\
\hline 1274 & 68.23 & 192.6 \\
\hline 1275 & 68.25 & 192.8 \\
\hline 1276 & 68.21 & 193.0 \\
\hline 1277 & 68.18 & 193.1 \\
\hline 1278 & 68.15 & 193.1 \\
\hline 1279 & 68.36 & 193.1 \\
\hline 1280 & 68.69 & 193.1 \\
\hline
\end{tabular}

\begin{tabular}{|c|c|c|}
\hline Time (s) & $\begin{array}{l}\text { Speed } \\
\text { (mph) }\end{array}$ & $\begin{array}{l}\text { Elevation } \\
\text { (m) }\end{array}$ \\
\hline 1281 & 69.06 & 193.1 \\
\hline 1282 & 68.89 & 193.1 \\
\hline 1283 & 68.85 & 193.4 \\
\hline 1284 & 68.79 & 193.6 \\
\hline 1285 & 68.75 & 193.9 \\
\hline 1286 & 68.70 & 194.1 \\
\hline 1287 & 68.59 & 194.3 \\
\hline 1288 & 68.54 & 194.4 \\
\hline 1289 & 68.54 & 194.5 \\
\hline 1290 & 68.54 & 194.6 \\
\hline 1291 & 68.45 & 194.6 \\
\hline 1292 & 68.04 & 194.6 \\
\hline 1293 & 67.62 & 194.6 \\
\hline 1294 & 67.46 & 194.7 \\
\hline 1295 & 67.38 & 194.7 \\
\hline 1296 & 67.30 & 194.7 \\
\hline 1297 & 67.23 & 194.7 \\
\hline 1298 & 66.82 & 194.8 \\
\hline 1299 & 66.47 & 195.0 \\
\hline 1300 & 66.27 & 195.2 \\
\hline 1301 & 66.23 & 195.4 \\
\hline 1302 & 66.23 & 195.6 \\
\hline 1303 & 66.23 & 195.8 \\
\hline 1304 & 66.38 & 196.0 \\
\hline 1305 & 66.57 & 196.1 \\
\hline 1306 & 66.80 & 196.1 \\
\hline 1307 & 66.97 & 196.2 \\
\hline 1308 & 68.00 & 195.7 \\
\hline 1309 & 69.09 & 195.2 \\
\hline 1310 & 69.01 & 195.2 \\
\hline 1311 & 68.97 & 195.2 \\
\hline 1312 & 68.96 & 195.2 \\
\hline 1313 & 68.96 & 195.0 \\
\hline 1314 & 68.99 & 194.8 \\
\hline 1315 & 69.00 & 194.5 \\
\hline 1316 & 69.00 & 194.2 \\
\hline 1317 & 68.99 & 193.9 \\
\hline 1318 & 68.95 & 193.5 \\
\hline 1319 & 68.95 & 193.1 \\
\hline 1320 & 68.95 & 192.6 \\
\hline 1321 & 68.78 & 192.6 \\
\hline 1322 & 68.54 & 192.1 \\
\hline 1323 & 68.34 & 191.6 \\
\hline 1324 & 67.99 & 191.3 \\
\hline 1325 & 68.00 & 191.3 \\
\hline 1326 & 68.07 & 190.3 \\
\hline 1327 & 69.33 & 190.3 \\
\hline 1328 & 70.63 & 189.3 \\
\hline 1329 & 70.75 & 189.3 \\
\hline 1330 & 70.92 & 189.6 \\
\hline 1331 & 71.33 & 189.8 \\
\hline 1332 & 71.68 & 190.1 \\
\hline 1333 & 71.63 & 190.4 \\
\hline 1334 & 70.93 & 190.7 \\
\hline 1335 & 70.85 & 190.9 \\
\hline 1336 & 70.29 & 190.9 \\
\hline
\end{tabular}




\begin{tabular}{|c|c|c|}
\hline Time (s) & $\begin{array}{l}\text { Speed } \\
\text { (mph) }\end{array}$ & $\begin{array}{l}\text { Elevation } \\
(\mathrm{m})\end{array}$ \\
\hline 1337 & 70.03 & 190.9 \\
\hline 1338 & 70.36 & 190.8 \\
\hline 1339 & 71.08 & 190.7 \\
\hline 1340 & 71.16 & 190.7 \\
\hline 1341 & 71.25 & 190.7 \\
\hline 1342 & 71.33 & 190.7 \\
\hline 1343 & 71.63 & 190.7 \\
\hline 1344 & 71.81 & 190.8 \\
\hline 1345 & 71.87 & 190.8 \\
\hline 1346 & 71.91 & 190.7 \\
\hline 1347 & 71.99 & 190.7 \\
\hline 1348 & 72.07 & 190.6 \\
\hline 1349 & 72.25 & 190.4 \\
\hline 1350 & 72.33 & 190.2 \\
\hline 1351 & 72.48 & 190.0 \\
\hline 1352 & 72.60 & 189.7 \\
\hline 1353 & 72.70 & 189.3 \\
\hline 1354 & 72.74 & 188.9 \\
\hline 1355 & 72.74 & 188.6 \\
\hline 1356 & 72.74 & 188.2 \\
\hline 1357 & 72.70 & 187.8 \\
\hline 1358 & 72.63 & 187.4 \\
\hline 1359 & 72.61 & 187.0 \\
\hline 1360 & 72.55 & 186.7 \\
\hline 1361 & 72.45 & 186.4 \\
\hline 1362 & 72.22 & 186.2 \\
\hline 1363 & 72.23 & 186.2 \\
\hline 1364 & 72.38 & 186.2 \\
\hline 1365 & 72.48 & 186.1 \\
\hline 1366 & 72.48 & 185.8 \\
\hline 1367 & 72.36 & 185.4 \\
\hline 1368 & 72.33 & 185.0 \\
\hline 1369 & 72.25 & 184.6 \\
\hline 1370 & 72.19 & 184.1 \\
\hline 1371 & 72.15 & 183.6 \\
\hline 1372 & 72.13 & 183.1 \\
\hline 1373 & 72.11 & 182.6 \\
\hline 1374 & 72.05 & 182.1 \\
\hline 1375 & 72.01 & 181.5 \\
\hline 1376 & 71.94 & 181.0 \\
\hline 1377 & 71.92 & 180.5 \\
\hline 1378 & 71.91 & 180.1 \\
\hline 1379 & 71.90 & 179.6 \\
\hline 1380 & 71.90 & 179.2 \\
\hline 1381 & 71.90 & 178.7 \\
\hline 1382 & 71.88 & 178.4 \\
\hline 1383 & 71.80 & 178.0 \\
\hline 1384 & 71.69 & 177.6 \\
\hline 1385 & 71.61 & 177.3 \\
\hline 1386 & 71.58 & 177.0 \\
\hline 1387 & 71.58 & 176.7 \\
\hline 1388 & 71.58 & 176.5 \\
\hline 1389 & 71.60 & 176.2 \\
\hline 1390 & 71.67 & 176.0 \\
\hline 1391 & 71.77 & 175.8 \\
\hline 1392 & 71.82 & 175.6 \\
\hline
\end{tabular}

\begin{tabular}{|lll|}
\hline Time (s) & Speed & Elevation \\
& (mph) & (m) \\
\hline 1393 & 71.89 & 175.4 \\
1394 & 71.92 & 175.2 \\
1395 & 71.94 & 175.0 \\
1396 & 71.95 & 174.9 \\
1397 & 71.96 & 174.7 \\
1398 & 71.95 & 174.3 \\
1399 & 71.94 & 174.2 \\
1400 & 71.92 & 174.0 \\
1401 & 71.89 & 173.8 \\
1402 & 71.89 & 173.6 \\
1403 & 71.89 & 173.4 \\
1404 & 71.88 & 173.3 \\
1405 & 71.97 & 173.1 \\
1406 & 72.06 & 173.0 \\
1407 & 72.05 & 173.0 \\
1408 & 72.04 & 172.8 \\
1409 & 72.09 & 172.3 \\
1410 & 72.16 & 171.6 \\
1411 & 72.18 & 170.9 \\
1412 & 72.18 & 170.1 \\
1413 & 72.19 & 169.3 \\
1414 & 72.09 & 168.6 \\
1415 & 71.96 & 167.8 \\
1416 & 71.82 & 167.2 \\
1417 & 71.66 & 166.6 \\
1418 & 71.61 & 166.1 \\
1419 & 71.46 & 165.7 \\
1420 & 71.34 & 165.4 \\
1421 & 71.32 & 165.2 \\
1422 & 71.32 & 165.1 \\
1423 & 71.40 & 165.0 \\
1424 & 71.57 & 165.0 \\
1425 & 71.80 & 165.0 \\
1426 & 72.07 & 165.0 \\
1427 & 72.18 & 165.0 \\
1428 & 72.18 & 165.0 \\
1429 & 72.13 & 164.9 \\
1430 & 72.07 & 164.9 \\
1431 & 72.04 & 164.8 \\
1432 & 72.02 & 164.7 \\
1433 & 72.01 & 164.7 \\
1434 & 72.02 & 164.6 \\
1435 & 72.09 & 164.4 \\
1436 & 72.17 & 164.4 \\
1437 & 72.24 & 164.3 \\
1438 & 72.27 & 164.2 \\
1439 & 72.18 & 164.2 \\
1440 & 72.00 & 164.1 \\
1441 & 71.81 & 164.1 \\
1442 & 71.89 & 164.1 \\
1443 & 71.96 & 164.1 \\
1444 & 72.04 & 164.1 \\
1445 & 72.12 & 164.1 \\
1446 & 72.24 & 164.1 \\
1447 & 72.43 & 164.1 \\
1448 & 72.62 & 164.0 \\
\hline & & \\
\hline
\end{tabular}




\begin{tabular}{|c|c|c|}
\hline Time (s) & $\begin{array}{l}\text { Speed } \\
\text { (mph) }\end{array}$ & $\begin{array}{l}\text { Elevation } \\
(\mathrm{m})\end{array}$ \\
\hline 1449 & 72.80 & 164.0 \\
\hline 1450 & 73.00 & 163.9 \\
\hline 1451 & 73.26 & 163.9 \\
\hline 1452 & 73.48 & 163.8 \\
\hline 1453 & 73.62 & 163.8 \\
\hline 1454 & 73.70 & 163.8 \\
\hline 1455 & 74.06 & 163.7 \\
\hline 1456 & 73.99 & 163.8 \\
\hline 1457 & 73.87 & 164.0 \\
\hline 1458 & 73.87 & 164.4 \\
\hline 1459 & 73.85 & 164.5 \\
\hline 1460 & 73.85 & 164.6 \\
\hline 1461 & 73.90 & 164.6 \\
\hline 1462 & 73.97 & 164.6 \\
\hline 1463 & 74.01 & 164.6 \\
\hline 1464 & 74.05 & 164.5 \\
\hline 1465 & 74.06 & 164.4 \\
\hline 1466 & 74.06 & 164.3 \\
\hline 1467 & 74.07 & 164.3 \\
\hline 1468 & 74.08 & 164.2 \\
\hline 1469 & 74.07 & 164.1 \\
\hline 1470 & 74.07 & 164.0 \\
\hline 1471 & 74.06 & 163.9 \\
\hline 1472 & 74.06 & 163.8 \\
\hline 1473 & 74.05 & 163.8 \\
\hline 1474 & 74.04 & 163.8 \\
\hline 1475 & 74.01 & 163.8 \\
\hline 1476 & 73.93 & 163.9 \\
\hline 1477 & 73.90 & 164.1 \\
\hline 1478 & 74.07 & 164.5 \\
\hline 1479 & 74.21 & 164.8 \\
\hline 1480 & 74.29 & 165.1 \\
\hline 1481 & 74.63 & 165.4 \\
\hline 1482 & 74.81 & 165.5 \\
\hline 1483 & 74.89 & 165.6 \\
\hline 1484 & 75.24 & 165.6 \\
\hline 1485 & 75.51 & 165.5 \\
\hline 1486 & 75.61 & 165.4 \\
\hline 1487 & 75.62 & 165.3 \\
\hline 1488 & 75.55 & 165.1 \\
\hline 1489 & 75.48 & 164.8 \\
\hline 1490 & 75.40 & 164.6 \\
\hline 1491 & 75.29 & 164.4 \\
\hline 1492 & 75.20 & 164.2 \\
\hline 1493 & 75.16 & 164.1 \\
\hline 1494 & 75.12 & 163.9 \\
\hline 1495 & 75.10 & 163.7 \\
\hline 1496 & 75.09 & 163.5 \\
\hline 1497 & 75.08 & 163.3 \\
\hline 1498 & 75.13 & 163.0 \\
\hline 1499 & 75.17 & 162.8 \\
\hline 1500 & 75.23 & 162.5 \\
\hline 1501 & 75.24 & 162.2 \\
\hline 1502 & 75.24 & 161.9 \\
\hline 1503 & 75.24 & 161.6 \\
\hline 1504 & 75.24 & 161.4 \\
\hline
\end{tabular}

\begin{tabular}{|c|c|c|}
\hline Time (s) & $\begin{array}{l}\text { Speed } \\
\text { (mph) }\end{array}$ & $\begin{array}{l}\text { Elevation } \\
\text { (m) }\end{array}$ \\
\hline 1505 & 75.25 & 161.1 \\
\hline 1506 & 75.26 & 160.9 \\
\hline 1507 & 75.27 & 160.6 \\
\hline 1508 & 75.30 & 160.4 \\
\hline 1509 & 75.32 & 160.3 \\
\hline 1510 & 75.32 & 160.2 \\
\hline 1511 & 75.32 & 160.1 \\
\hline 1512 & 75.30 & 160.1 \\
\hline 1513 & 74.33 & 160.1 \\
\hline 1514 & 73.29 & 160.1 \\
\hline 1515 & 72.86 & 160.1 \\
\hline 1516 & 72.26 & 160.1 \\
\hline 1517 & 71.69 & 160.1 \\
\hline 1518 & 71.13 & 160.0 \\
\hline 1519 & 70.63 & 160.0 \\
\hline 1520 & 70.55 & 160.0 \\
\hline 1521 & 70.47 & 160.5 \\
\hline 1522 & 70.52 & 161.0 \\
\hline 1523 & 70.53 & 161.5 \\
\hline 1524 & 70.53 & 161.5 \\
\hline 1525 & 70.52 & 161.4 \\
\hline 1526 & 70.57 & 161.3 \\
\hline 1527 & 70.61 & 161.1 \\
\hline 1528 & 70.65 & 160.8 \\
\hline 1529 & 70.70 & 160.4 \\
\hline 1530 & 70.75 & 159.9 \\
\hline 1531 & 70.75 & 159.3 \\
\hline 1532 & 70.63 & 158.0 \\
\hline 1533 & 70.54 & 157.3 \\
\hline 1534 & 70.50 & 156.5 \\
\hline 1535 & 70.41 & 155.8 \\
\hline 1536 & 70.33 & 154.3 \\
\hline 1537 & 70.19 & 153.7 \\
\hline 1538 & 69.45 & 153.1 \\
\hline 1539 & 68.08 & 152.6 \\
\hline 1540 & 67.49 & 152.1 \\
\hline 1541 & 67.28 & 151.7 \\
\hline 1542 & 67.22 & 151.4 \\
\hline 1543 & 67.22 & 150.8 \\
\hline 1544 & 67.56 & 150.6 \\
\hline 1545 & 67.96 & 150.4 \\
\hline 1546 & 68.02 & 150.2 \\
\hline 1547 & 68.03 & 150.0 \\
\hline 1548 & 68.03 & 149.8 \\
\hline 1549 & 67.83 & 149.4 \\
\hline 1550 & 67.19 & 149.2 \\
\hline 1551 & 66.02 & 149.0 \\
\hline 1552 & 64.82 & 148.8 \\
\hline 1553 & 64.19 & 148.6 \\
\hline 1554 & 63.73 & 148.4 \\
\hline 1555 & 62.36 & 148.2 \\
\hline 1556 & 62.32 & 148.2 \\
\hline 1557 & 62.45 & 148.2 \\
\hline 1558 & 62.56 & 147.8 \\
\hline 1559 & 62.56 & 147.4 \\
\hline 1560 & 62.56 & 146.9 \\
\hline
\end{tabular}




\begin{tabular}{|c|c|c|}
\hline Time (s) & $\begin{array}{l}\text { Speed } \\
\text { (mph) }\end{array}$ & $\begin{array}{l}\text { Elevation } \\
(\mathrm{m})\end{array}$ \\
\hline 1561 & 62.44 & 146.5 \\
\hline 1562 & 62.02 & 146.0 \\
\hline 1563 & 61.88 & 145.6 \\
\hline 1564 & 61.88 & 145.1 \\
\hline 1565 & 61.88 & 144.7 \\
\hline 1566 & 62.25 & 144.3 \\
\hline 1567 & 62.60 & 143.9 \\
\hline 1568 & 62.62 & 143.6 \\
\hline 1569 & 62.62 & 143.3 \\
\hline 1570 & 62.79 & 143.1 \\
\hline 1571 & 62.81 & 142.9 \\
\hline 1572 & 62.81 & 142.8 \\
\hline 1573 & 62.89 & 142.6 \\
\hline 1574 & 63.25 & 142.5 \\
\hline 1575 & 63.50 & 142.4 \\
\hline 1576 & 63.72 & 142.4 \\
\hline 1577 & 63.30 & 142.4 \\
\hline 1578 & 63.26 & 142.4 \\
\hline 1579 & 63.27 & 142.2 \\
\hline 1580 & 63.12 & 141.9 \\
\hline 1581 & 62.28 & 141.6 \\
\hline 1582 & 62.16 & 141.3 \\
\hline 1583 & 62.16 & 140.9 \\
\hline 1584 & 62.16 & 140.5 \\
\hline 1585 & 62.63 & 140.5 \\
\hline 1586 & 62.89 & 140.8 \\
\hline 1587 & 63.06 & 140.8 \\
\hline 1588 & 63.15 & 140.8 \\
\hline 1589 & 63.24 & 140.8 \\
\hline 1590 & 63.32 & 141.1 \\
\hline 1591 & 63.58 & 141.1 \\
\hline 1592 & 64.03 & 141.3 \\
\hline 1593 & 63.42 & 141.7 \\
\hline 1594 & 62.83 & 142.4 \\
\hline 1595 & 62.77 & 143.1 \\
\hline 1596 & 62.29 & 143.7 \\
\hline 1597 & 61.79 & 144.2 \\
\hline 1598 & 61.44 & 144.5 \\
\hline 1599 & 61.03 & 144.5 \\
\hline 1600 & 60.69 & 144.4 \\
\hline 1601 & 60.68 & 144.4 \\
\hline 1602 & 60.68 & 144.3 \\
\hline 1603 & 60.75 & 144.3 \\
\hline 1604 & 61.45 & 144.3 \\
\hline 1605 & 62.11 & 144.2 \\
\hline 1606 & 62.03 & 144.2 \\
\hline 1607 & 61.38 & 144.5 \\
\hline 1608 & 60.89 & 145.2 \\
\hline 1609 & 60.53 & 145.8 \\
\hline 1610 & 60.11 & 146.5 \\
\hline 1611 & 59.85 & 147.1 \\
\hline 1612 & 59.59 & 147.7 \\
\hline 1613 & 59.52 & 148.3 \\
\hline 1614 & 59.52 & 148.8 \\
\hline 1615 & 59.52 & 149.3 \\
\hline 1616 & 59.58 & 149.7 \\
\hline
\end{tabular}

\begin{tabular}{|lll|}
\hline Time (s) & Speed & Elevation \\
& (mph) & (m) \\
\hline 1617 & 59.75 & 150.1 \\
1618 & 60.01 & 150.4 \\
1619 & 60.28 & 150.6 \\
1620 & 60.39 & 150.8 \\
1621 & 60.60 & 150.8 \\
1622 & 60.84 & 150.9 \\
1623 & 60.90 & 151.3 \\
1624 & 60.90 & 152.1 \\
1625 & 61.05 & 153.0 \\
1626 & 61.26 & 154.0 \\
1627 & 61.28 & 155.1 \\
1628 & 61.28 & 156.1 \\
1629 & 61.28 & 157.2 \\
1630 & 61.26 & 158.2 \\
1631 & 61.20 & 159.1 \\
1632 & 61.05 & 159.9 \\
1633 & 61.04 & 160.6 \\
1634 & 61.04 & 161.3 \\
1635 & 61.05 & 161.8 \\
1636 & 61.06 & 162.3 \\
1637 & 61.06 & 162.7 \\
1638 & 61.06 & 162.9 \\
1639 & 60.91 & 162.9 \\
1640 & 60.36 & 162.9 \\
1641 & 60.34 & 163.0 \\
1642 & 60.34 & 163.1 \\
1643 & 60.45 & 163.2 \\
1644 & 60.81 & 163.4 \\
1645 & 61.11 & 163.6 \\
1646 & 61.36 & 163.8 \\
1647 & 61.53 & 164.1 \\
1648 & 61.61 & 164.3 \\
1649 & 61.64 & 164.4 \\
1650 & 61.69 & 164.6 \\
1651 & 61.85 & 164.7 \\
1652 & 61.63 & 164.7 \\
1653 & 61.54 & 164.7 \\
1654 & 61.50 & 164.7 \\
1655 & 61.49 & 164.6 \\
1656 & 61.49 & 164.6 \\
1657 & 60.77 & 164.5 \\
1658 & 60.59 & 164.5 \\
1659 & 60.50 & 164.3 \\
1660 & 60.35 & 164.2 \\
1661 & 60.14 & 164.0 \\
1662 & 59.99 & 163.8 \\
1663 & 59.88 & 163.6 \\
1664 & 59.71 & 163.4 \\
1665 & 59.59 & 163.2 \\
1666 & 59.41 & 163.2 \\
1667 & 59.11 & 163.5 \\
1668 & 58.90 & 164.0 \\
1669 & 58.77 & 164.7 \\
1670 & 58.57 & 165.4 \\
1671 & 58.44 & 166.2 \\
1672 & 58.42 & 166.9 \\
\hline & & \\
\hline
\end{tabular}




\begin{tabular}{|c|c|c|}
\hline Time (s) & $\begin{array}{l}\text { Speed } \\
\text { (mph) }\end{array}$ & $\begin{array}{l}\text { Elevation } \\
(\mathrm{m})\end{array}$ \\
\hline 1673 & 58.42 & 167.6 \\
\hline 1674 & 58.44 & 168.2 \\
\hline 1675 & 58.63 & 168.8 \\
\hline 1676 & 58.74 & 169.1 \\
\hline 1677 & 58.75 & 169.7 \\
\hline 1678 & 58.75 & 170.3 \\
\hline 1679 & 58.69 & 171.0 \\
\hline 1680 & 58.50 & 171.9 \\
\hline 1681 & 58.22 & 172.9 \\
\hline 1682 & 57.98 & 173.9 \\
\hline 1683 & 58.23 & 174.9 \\
\hline 1684 & 57.71 & 176.0 \\
\hline 1685 & 57.27 & 176.8 \\
\hline 1686 & 57.20 & 176.8 \\
\hline 1687 & 57.11 & 176.8 \\
\hline 1688 & 56.67 & 176.8 \\
\hline 1689 & 56.41 & 176.7 \\
\hline 1690 & 56.18 & 176.5 \\
\hline 1691 & 55.98 & 176.2 \\
\hline 1692 & 56.16 & 175.8 \\
\hline 1693 & 56.30 & 175.3 \\
\hline 1694 & 56.66 & 174.9 \\
\hline 1695 & 56.99 & 174.6 \\
\hline 1696 & 57.41 & 174.4 \\
\hline 1697 & 57.37 & 174.4 \\
\hline 1698 & 56.85 & 174.9 \\
\hline 1699 & 56.78 & 175.5 \\
\hline 1700 & 56.79 & 176.9 \\
\hline 1701 & 56.80 & 177.3 \\
\hline 1702 & 56.91 & 177.3 \\
\hline 1703 & 56.99 & 177.6 \\
\hline 1704 & 57.10 & 177.9 \\
\hline 1705 & 57.02 & 177.9 \\
\hline 1706 & 56.93 & 178.6 \\
\hline 1707 & 56.85 & 179.0 \\
\hline 1708 & 56.76 & 179.4 \\
\hline 1709 & 56.59 & 179.5 \\
\hline 1710 & 56.52 & 179.6 \\
\hline 1711 & 56.44 & 179.6 \\
\hline 1712 & 56.35 & 179.9 \\
\hline 1713 & 56.27 & 180.3 \\
\hline 1714 & 56.18 & 180.6 \\
\hline 1715 & 56.09 & 181.4 \\
\hline 1716 & 56.01 & 182.7 \\
\hline 1717 & 55.87 & 183.3 \\
\hline 1718 & 55.81 & 183.9 \\
\hline 1719 & 55.77 & 183.9 \\
\hline 1720 & 55.20 & 183.9 \\
\hline 1721 & 54.45 & 183.1 \\
\hline 1722 & 53.54 & 182.4 \\
\hline 1723 & 52.74 & 181.9 \\
\hline 1724 & 52.82 & 181.9 \\
\hline 1725 & 52.90 & 181.9 \\
\hline 1726 & 52.94 & 181.9 \\
\hline 1727 & 52.91 & 181.8 \\
\hline 1728 & 52.90 & 181.8 \\
\hline
\end{tabular}

\begin{tabular}{|lll|}
\hline Time (s) & Speed & Elevation \\
& (mph) & (m) \\
\hline 1729 & 52.90 & 181.7 \\
1730 & 52.49 & 181.7 \\
1731 & 51.73 & 181.6 \\
1732 & 50.81 & 181.5 \\
1733 & 49.81 & 181.5 \\
1734 & 49.59 & 181.4 \\
1735 & 49.59 & 181.4 \\
1736 & 49.59 & 181.4 \\
1737 & 49.81 & 181.4 \\
1738 & 50.17 & 181.4 \\
1739 & 50.60 & 181.4 \\
1740 & 50.93 & 181.3 \\
1741 & 51.16 & 181.3 \\
1742 & 51.17 & 181.2 \\
1743 & 51.17 & 181.1 \\
1744 & 51.11 & 181.0 \\
1745 & 50.99 & 180.8 \\
1746 & 50.98 & 180.6 \\
1747 & 50.98 & 180.4 \\
1748 & 51.21 & 180.2 \\
1749 & 51.70 & 179.9 \\
1750 & 52.29 & 179.7 \\
1751 & 52.82 & 179.5 \\
1752 & 53.30 & 179.4 \\
1753 & 53.63 & 179.2 \\
1754 & 53.94 & 179.2 \\
1755 & 54.13 & 179.1 \\
1756 & 53.73 & 179.1 \\
1757 & 53.72 & 178.3 \\
1758 & 53.71 & 177.6 \\
1759 & 53.42 & 177.0 \\
1760 & 53.33 & 176.2 \\
1761 & 53.17 & 175.5 \\
1762 & 53.96 & 174.6 \\
1763 & 54.23 & 173.5 \\
1764 & 54.31 & 172.2 \\
1765 & 54.37 & 170.8 \\
1766 & 54.42 & 168.1 \\
1767 & 54.47 & 166.8 \\
1768 & 54.98 & 165.7 \\
1769 & 55.60 & 164.7 \\
1770 & 55.59 & 163.8 \\
1771 & 55.38 & 161.4 \\
1772 & 55.11 & 161.1 \\
1773 & 54.98 & 161.2 \\
1774 & 54.65 & 161.2 \\
1775 & 54.52 & 161.2 \\
1776 & 54.45 & 161.3 \\
1777 & 54.36 & 161.3 \\
1778 & 54.77 & 161.4 \\
1779 & 55.17 & 161.5 \\
1780 & 55.52 & 161.6 \\
1781 & 55.60 & 161.6 \\
1782 & 55.68 & 161.6 \\
1783 & 55.77 & 161.6 \\
1784 & 55.85 & 161.6 \\
\hline & & \\
\hline
\end{tabular}




\begin{tabular}{|c|c|c|}
\hline Time (s) & $\begin{array}{l}\text { Speed } \\
\text { (mph) }\end{array}$ & $\begin{array}{l}\text { Elevation } \\
(\mathrm{m})\end{array}$ \\
\hline 1785 & 55.94 & 161.6 \\
\hline 1786 & 56.47 & 161.5 \\
\hline 1787 & 57.05 & 161.2 \\
\hline 1788 & 57.54 & 160.9 \\
\hline 1789 & 58.01 & 160.6 \\
\hline 1790 & 58.36 & 160.3 \\
\hline 1791 & 58.58 & 160.0 \\
\hline 1792 & 58.67 & 159.8 \\
\hline 1793 & 58.67 & 159.9 \\
\hline 1794 & 58.67 & 160.2 \\
\hline 1795 & 58.69 & 160.6 \\
\hline 1796 & 58.85 & 161.0 \\
\hline 1797 & 59.06 & 161.4 \\
\hline 1798 & 59.34 & 161.7 \\
\hline 1799 & 59.66 & 161.8 \\
\hline 1800 & 60.02 & 161.9 \\
\hline 1801 & 60.45 & 161.9 \\
\hline 1802 & 60.95 & 161.9 \\
\hline 1803 & 61.37 & 161.9 \\
\hline 1804 & 60.61 & 161.9 \\
\hline 1805 & 60.59 & 161.9 \\
\hline 1806 & 60.58 & 162.3 \\
\hline 1807 & 60.57 & 162.8 \\
\hline 1808 & 60.60 & 163.3 \\
\hline 1809 & 60.61 & 163.7 \\
\hline 1810 & 60.66 & 164.2 \\
\hline 1811 & 60.68 & 164.6 \\
\hline 1812 & 60.71 & 165.0 \\
\hline 1813 & 60.77 & 165.4 \\
\hline 1814 & 60.86 & 165.7 \\
\hline 1815 & 61.02 & 166.0 \\
\hline 1816 & 60.95 & 166.0 \\
\hline 1817 & 60.95 & 165.9 \\
\hline 1818 & 60.95 & 165.8 \\
\hline 1819 & 60.73 & 165.8 \\
\hline 1820 & 60.42 & 165.7 \\
\hline 1821 & 59.91 & 165.7 \\
\hline 1822 & 59.66 & 165.7 \\
\hline 1823 & 59.06 & 165.7 \\
\hline 1824 & 59.07 & 165.7 \\
\hline 1825 & 59.27 & 165.8 \\
\hline 1826 & 59.46 & 165.8 \\
\hline 1827 & 59.61 & 165.9 \\
\hline 1828 & 58.99 & 165.9 \\
\hline 1829 & 58.99 & 165.9 \\
\hline 1830 & 58.99 & 165.9 \\
\hline 1831 & 58.99 & 165.6 \\
\hline 1832 & 58.89 & 165.4 \\
\hline 1833 & 58.89 & 165.4 \\
\hline 1834 & 59.06 & 165.4 \\
\hline 1835 & 59.17 & 165.4 \\
\hline 1836 & 59.23 & 165.3 \\
\hline 1837 & 59.23 & 165.2 \\
\hline 1838 & 59.23 & 165.1 \\
\hline 1839 & 59.30 & 165.0 \\
\hline 1840 & 59.24 & 165.0 \\
\hline
\end{tabular}

\begin{tabular}{|lll|}
\hline Time (s) & Speed & Elevation \\
& (mph) & (m) \\
\hline 1841 & 59.17 & 165.0 \\
1842 & 59.15 & 165.0 \\
1843 & 59.11 & 165.0 \\
1844 & 57.81 & 165.0 \\
1845 & 57.73 & 165.0 \\
1846 & 57.65 & 165.0 \\
1847 & 57.37 & 165.0 \\
1848 & 57.37 & 165.1 \\
1849 & 57.51 & 165.5 \\
1850 & 57.60 & 166.0 \\
1851 & 57.63 & 166.5 \\
1852 & 57.63 & 167.1 \\
1853 & 57.63 & 167.7 \\
1854 & 57.52 & 168.3 \\
1855 & 57.44 & 168.9 \\
1856 & 57.35 & 170.1 \\
1857 & 57.25 & 170.7 \\
1858 & 57.10 & 171.3 \\
1859 & 55.85 & 171.9 \\
1860 & 54.99 & 172.5 \\
1861 & 54.80 & 173.1 \\
1862 & 54.39 & 173.6 \\
1863 & 53.29 & 174.1 \\
1864 & 52.95 & 174.5 \\
1865 & 52.50 & 175.0 \\
1866 & 51.82 & 175.5 \\
1867 & 51.23 & 176.0 \\
1868 & 50.55 & 176.6 \\
1869 & 50.51 & 177.0 \\
1870 & 50.26 & 177.3 \\
1871 & 48.86 & 177.7 \\
1872 & 47.43 & 178.3 \\
1873 & 46.61 & 178.9 \\
1874 & 45.97 & 179.4 \\
1875 & 46.94 & 179.4 \\
1876 & 45.86 & 179.4 \\
1877 & 45.72 & 179.5 \\
1878 & 45.71 & 180.0 \\
1879 & 46.39 & 180.3 \\
1880 & 46.77 & 180.6 \\
1881 & 47.05 & 181.0 \\
1882 & 46.96 & 181.4 \\
1883 & 46.99 & 181.4 \\
1884 & 47.07 & 181.4 \\
1885 & 47.16 & 181.4 \\
1886 & 47.05 & 181.4 \\
1887 & 47.59 & 181.4 \\
1888 & 48.13 & 181.9 \\
1889 & 48.73 & 182.4 \\
1890 & 48.99 & 183.1 \\
1891 & 48.99 & 183.8 \\
1892 & 48.99 & 184.5 \\
1893 & 48.11 & 185.1 \\
1894 & 45.96 & 185.6 \\
1895 & 43.47 & 185.9 \\
1896 & 41.26 & 186.2 \\
\hline & & \\
\hline
\end{tabular}




\begin{tabular}{|lll|}
\hline Time (s) & Speed & Elevation \\
& (mph) & (m) \\
\hline 1897 & 40.55 & 186.5 \\
1898 & 40.51 & 186.9 \\
1899 & 38.97 & 187.4 \\
1900 & 38.57 & 188.0 \\
1901 & 36.82 & 188.4 \\
1902 & 36.50 & 188.8 \\
1903 & 36.49 & 189.2 \\
1904 & 35.83 & 189.6 \\
1905 & 35.75 & 189.9 \\
1906 & 34.96 & 190.2 \\
1907 & 34.73 & 190.4 \\
1908 & 34.69 & 190.6 \\
1909 & 33.27 & 190.8 \\
1910 & 33.10 & 191.0 \\
1911 & 32.08 & 191.1 \\
1912 & 32.04 & 191.3 \\
1913 & 31.75 & 191.4 \\
1914 & 30.84 & 191.6 \\
1915 & 30.48 & 191.7 \\
1916 & 30.41 & 191.9 \\
1917 & 29.77 & 192.1 \\
1918 & 29.15 & 192.2 \\
1919 & 29.06 & 192.4 \\
1920 & 28.52 & 192.6 \\
1921 & 28.26 & 192.8 \\
1922 & 28.09 & 193.0 \\
1923 & 27.30 & 193.2 \\
1924 & 27.26 & 193.4 \\
1925 & 26.43 & 193.6 \\
1926 & 26.39 & 193.8 \\
1927 & 25.22 & 194.0 \\
1928 & 23.53 & 194.2 \\
1929 & 23.49 & 194.3 \\
1930 & 22.08 & 194.5 \\
1931 & 22.04 & 194.7 \\
1932 & 21.91 & 194.9 \\
1933 & 21.87 & 195.0 \\
1934 & 20.79 & 195.2 \\
1935 & 20.26 & 195.2 \\
1936 & 20.21 & 195.2 \\
1937 & 19.50 & 195.2 \\
1938 & 19.37 & 195.2 \\
1939 & 19.05 & 195.2 \\
1940 & 18.61 & 195.2 \\
1941 & 18.52 & 195.2 \\
1942 & 17.46 & 195.2 \\
1943 & 16.75 & 195.2 \\
1944 & 16.14 & 195.2 \\
1945 & 15.55 & 195.2 \\
1946 & 15.52 & 195.2 \\
1947 & 14.95 & 195.2 \\
1948 & 14.81 & 195.2 \\
1949 & 13.71 & 195.2 \\
1950 & 12.27 & 195.2 \\
1951 & 12.23 & 195.2 \\
\hline
\end{tabular}

\begin{tabular}{|lll|}
\hline Time (s) & $\begin{array}{l}\text { Speed } \\
\text { (mph) }\end{array}$ & Elevation \\
& (m) \\
\hline 1952 & 11.50 & 195.2 \\
1953 & 10.97 & 195.2 \\
1954 & 9.79 & 195.2 \\
1955 & 8.25 & 195.2 \\
1956 & 7.39 & 195.2 \\
1957 & 6.65 & 195.2 \\
1958 & 5.59 & 195.2 \\
1959 & 4.66 & 195.2 \\
1960 & 3.99 & 195.2 \\
1961 & 2.91 & 195.2 \\
1962 & 1.65 & 195.2 \\
1963 & 0.26 & 195.2 \\
1964 & 0.00 & 195.2 \\
1965 & 0.00 & 195.2 \\
1966 & 0.00 & 195.2 \\
1967 & 0.00 & 195.2 \\
1968 & 0.00 & 195.2 \\
1969 & 0.00 & 195.2 \\
1970 & 0.00 & 195.2 \\
1971 & 0.00 & 195.2 \\
1972 & 0.00 & 195.2 \\
1973 & 0.00 & 195.2 \\
1974 & 0.00 & 195.2 \\
1975 & 0.00 & 195.2 \\
1976 & 0.00 & 195.2 \\
1977 & 0.00 & 195.2 \\
1978 & 0.00 & 195.2 \\
1979 & 0.00 & 195.2 \\
1980 & 0.00 & 195.2 \\
1981 & 0.00 & 195.2 \\
1982 & 0.00 & 195.2 \\
1983 & 0.00 & 195.2 \\
1984 & 0.00 & 195.2 \\
1985 & 0.00 & 195.2 \\
1986 & 0.00 & 195.2 \\
1987 & 0.00 & 195.2 \\
1988 & 0.00 & 195.2 \\
1989 & 0.00 & 195.2 \\
1990 & 0.00 & 195.2 \\
1991 & 0.00 & 195.2 \\
1992 & 0.00 & 195.2 \\
1993 & 0.00 & 195.2 \\
1994 & 0.00 & 195.2 \\
1995 & 0.00 & 195.2 \\
1996 & 0.00 & 195.2 \\
1997 & 0.00 & 195.2 \\
\hline & & \\
\hline
\end{tabular}

